\title{
Enhancement of RNA Splicing by the Nutrient-Regulated Splicing Factor SRSF3
}

Amanda L. Suchanek

Follow this and additional works at: https://researchrepository.wvu.edu/etd

\section{Recommended Citation}

Suchanek, Amanda L., "Enhancement of RNA Splicing by the Nutrient-Regulated Splicing Factor SRSF3" (2015). Graduate Theses, Dissertations, and Problem Reports. 6740.

https://researchrepository.wvu.edu/etd/6740

This Dissertation is protected by copyright and/or related rights. It has been brought to you by the The Research Repository @ WVU with permission from the rights-holder(s). You are free to use this Dissertation in any way that is permitted by the copyright and related rights legislation that applies to your use. For other uses you must obtain permission from the rights-holder(s) directly, unless additional rights are indicated by a Creative Commons license in the record and/ or on the work itself. This Dissertation has been accepted for inclusion in WVU Graduate Theses, Dissertations, and Problem Reports collection by an authorized administrator of The Research Repository @ WVU.

For more information, please contact researchrepository@mail.wvu.edu. 


\title{
Enhancement of RNA Splicing by the Nutrient-Regulated Splicing Factor SRSF3.
}

\author{
Amanda L. Suchanek
}

Dissertation submitted to the

School of Medicine

at West Virginia University

in partial fulfillment of the requirements

for the degree of

Doctor of Philosophy

in

Biochemistry \& Molecular Biology

\author{
Committee Members \\ Lisa Salati, Ph.D., Chair \\ John Hollander, Ph.D. \\ J. Michael Ruppert, M.D., Ph.D. \\ Maxim Sokolov, Ph.D. \\ Yehenew Agazie, Ph.D. \\ Graduate Program in Biochemistry \\ West Virginia University School of Medicine \\ Morgantown, West Virginia \\ 2015
}

Keywords: SRSF3, G6PD, RNA splicing, hepatocytes, adenovirus, intron retention

(c) 2015 Amanda Suchanek 


\section{ABSTRACT \\ Enhancement of RNA Splicing by the Nutrient Regulated Splicing Factor, SRSF3}

\section{Amanda L. Suchanek}

Nutritional status is a powerful regulator of intracellular function. Dietary status can increase or decrease the rate of RNA splicing, thereby affecting gene expression; however, few molecular mechanisms have been identified that are responsible for this type of regulation.

The glucose-6-phosphate dehydrogenase (G6PD) gene has provided a useful tool for the study of nutrient-regulated splicing because accumulation of G6PD mRNA is dependent solely on changes in the rate of mRNA splicing in response to nutritional stimuli, consistent with its enzymatic role of converting dietary energy to fatty acids. Treatment of primary hepatocytes in culture with insulin or feeding a high-carbohydrate, low fat diet to rodents increases splicing by 7- and 15-fold, respectively, and increases the cellular content of G6PD. In contrast, starvation or treatment of primary hepatocytes in culture with polyunsaturated fatty acids reduces G6PD mRNA splicing by $70 \%$ or more. The unspliced RNA is degraded in the nucleus, thus reducing expression of the G6PD enzyme. Our laboratory identified a splicing regulatory element within exon 12 of the G6PD transcript is required for splicingrelated changes in G6PD expression in response to nutrient stimuli. This element is bound by two splicing regulatory proteins, SRSF3 and hnRNP K.

To understand the mechanism of action of these proteins, we used a loss-of-function approach. SiRNA-mediated depletion of SRSF3 significantly decreased G6PD splicing and expression; in contrast, depletion of hnRNP K enhanced both splicing and mRNA accumulation. Consistent with their apparent roles as enhancers or silencers of splicing, respectively, the results of RNA immunoprecipitation (RIP) indicated binding of SRSF3 to exon 12 was enhanced 6-fold in the livers of refed mice and nearly undetectable in starved mice. Conversely, binding of hnRNP $\mathrm{K}$ to the regulatory element was increased 12.5 -fold in the livers of starved mice and barely detectable in the livers of refed mice. Furthermore, RNA EMSA using purified SRSF3 and hnRNP K recombinant proteins, we demonstrated that SRSF3 and hnRNP K compete for binding to the same sequences within the regulatory element, which we hypothesize is a bifunctional ESE/ESS element. Thus, mutually exclusive binding of SRSF3 and hnRNP K to this regulatory element mediates the nutrient regulation of G6PD mRNA splicing and establishes a new intracellular mechanism for nutrient regulation of gene expression. We hypothesize that nutrient regulation of splicing is not unique to the G6PD mRNA, and thus the experiments described herein are focused identifying additional genes that are regulated by changes in alternative splicing in response to nutrient availability. 


\section{ACKNOWLEDGMENTS}

I would like to thank all of the people who have stood by and supported me through this incredible journey. I'd like to thank my dissertation committee, and especially my advisor, Lisa Salati, for giving me a chance and for giving me the tools to be a successful research scientist. I'd also like to thank Brad Hillgartner, Roberta Leonardi, and Peter Stoilov for their advice and criticism during lab meetings and other presentations that have helped me become a stronger public speaker.

I can say without hesitation that I would not have reached this point without the steadfast support of my family and friends. l'd first like to thank my father and mother, Joe \& Cindy Suchanek, and my sister, Michelle Navarro, for always encouraging me to pursue my goals, for their emotional support and calming influences during the ups and downs of these last several years. I'd also like to thank my "Pennsylvania family," especially my uncle Gary and aunt Cathy Robinson, cousins Cristen and Cris Lambert, and David, Tina, Jon, Jessi, and Courtney for their support (and Thanksgiving/Easter dinners!) while I have been pursuing this degree.

I would like to thank my former lab mates Holly and Travis Cyphert for their friendship, advice, and our always exciting scientific discussions. I'd like to thank Jess Gibat and Kim Alonge for their friendship and support here in the biochemistry department. Our alwayscolorful conversations, scientific and otherwise, have gone a long way in keeping me sane. Thank you to my friends Gina Mazzetti, Rosemary and Jerry Munsey, without whom these final months spent finishing this degree would have been next to impossible. Finally, thank you to my other "sisters," Alisa Elliott, Bahar Mihalcin, and Courtney Newhouse. Their unwavering support, encouragement, friendship, and laughter has kept me going through some of the toughest years of my life. 
I. RNA PROCESSING

1.1 INTRODUCTION

1.2 CAPPING

1.3 POLYADENYLATION

1.4 SPLICING

1.5 POST-SPLICING

1.6 ADDITIONAL SPLICING REGULATORY SEQUENCES

II. TYPES OF SPLICING

2.1 INTRODUCTION

2.2 ALTERNATIVE 5' OR 3' SPLICE SITE CHOICE 11

2.3 CASSETTE EXON SKIPPING/INCLUSION 15

2.4 INTRON RETENTION

III. REGULATION OF SPLICING

3.1 INTRODUCTION

3.2 hnRNPs

3.3 SR PROTEINS

3.4 REGULATION OF SR PROTEINS

3.5 SRSF3 AND REGULATION OF G6PD
17

18

20

22

24

26

27

V. REFERENCES

CHAPTER 2: SERINE ARGININE SPLICING FACTOR 3 (SRSF3) IS INVOLVED IN ENHANCED SPLICING OF GLUCOSE-6PHOSPHATE DEHYDROGENASE (G6PD) RNA IN RESPONSE TO NUTRIENTS AND HORMONES IN LIVER

CHAPTER 3: CONSTRUCTION AND EVALUATION OF AN ADENOVIRAL VECTOR FOR THE LIVER-SPECIFIC EXPRESSION OF THE SERINE/ARGININE-RICH SPLICING FACTOR, SRSF3 
CHAPTER 4: INTRON RETENTION IS NOT A MAJOR REGULATOR OF HEPATIC GENE EXPRESSION

CHAPTER 5: SUMMARY

APPENDIX:

I. CLIP-SEQ PROTOCOL

153

II. DUAL ISOLATION OF PROTEIN AND RNA FROM CELLS IN CULTURE

III. RNA IMMUNOPRECIPITATION PROTOCOL 


\section{LIST OF FIGURES}

\section{CHAPTER 1:}

Figure 1: mRNA and the spliceosome.

Figure 2: Splicing catalysis.

7

Figure 3: Types of splicing.

Figure 4: The SR protein family.

\section{CHAPTER 2:}

Figure 1. Insulin and arachidonic acid regulate the amount of phosphorylated SR proteins in the nuclei of primary rat hepatocytes.

82

Figure 2. Refeeding increases the binding of phosphorylated SR proteins to the regulatory element of G6PD exon 12 in vivo.

83

Figure 3. SRSF3 and SRSF4 bind to the splicing regulatory element.

Figure 4. Purified SRSF3 binds to the regulatory element in exon 12.

Figure 5. SiRNA-mediated depletion of SRSF3 reduces the splicing of a G6PD reporter and the endogenous G6PD mRNA.

Figure 6. SiRNA-mediated depletion of SRSF3 reduces splicing of G6PD in HeLa cells.

Figure 7. SRSF3 specifically binds to the splicing regulatory element in vivo and refeeding enhances the binding of SRSF3 to the splicing regulatory element in mouse liver.

\section{CHAPTER 3:}

Figure 1: Cloning schemes used to construct adenoviral shuttle vectors.

Figure 2: CMV-driven FLAG-SRSF3 is highly expressed in HEK293T packaging cells while albumin-driven FLAG-SRSF3 is not.

Figure 3: FLAG-SRSF3 is expressed in HepG2 cells infected with albumin FLAG-SRSF3 adenovirus. 
Figure 4: FLAG-SRSF3 is detected in primary rat hepatocytes infected with albumin FLAG-SRSF3 adenovirus.

Figure 5: Overexpression of FLAG-SRSF3 regulates mRNA abundance of mRNA targets.

\section{CHAPTER 4:}

Figure 1: Experimental approach.

a. Mouse experiment workflow.

b. Agarose gel of total RNA collected from whole mouse liver.

c. Representative BioAnalyzer traces of total RNA collected from whole mouse liver.

Figure 2: qRT-PCR analysis of genes known to respond to starvation and refeeding.

Table 1a: Intron Retention Events (sorted by significance)

Table 1b: Intron Retention Events (sorted alphabetically)

Table 2a: Exon Skipping Events (sorted by significance)

Table 3a: Mutually Exclusive Exons (sorted by significance)

Table 4a: Alternate 3'-splice site usage (sorted by significance)

Table 4b: Alternate 3'-splice site usage (sorted alphabetically)

Table 5a: Alternate 5'-splice site usage (sorted by significance)

Table 5b: Alternate 5'-splice site usage (sorted alphabetically)

Table 6a: Overall Expression Changes (sorted by significance)

Table 6b: Overall Expression Changes (sorted alphabetically) 


\section{LIST OF ABBREVIATIONS}

Pol II

CTD

CBC

snRNP

EJC

NMD

ESE

ISE

ESS

ISS

SR protein

SRSF

hnRNP

G6PD

RRM

RS domain

RIP

CLIP

MOI

AAV

SREBP-1

ME1

NF-KB

GusB

FASN

PEPCK

SCD1

FDR

IncLD

RIN
RNA polymerase II

C-terminal domain of RNA Pol II

Cap binding complex

Small nuclear ribonucleoprotein particle

Exon junction complex

Nonsense-mediated decay

Exonic splicing enhancer

Intronic splicing enhancer

Exonic splicing silencer

Intronic splicing silencer

Serine/arginine-rich protein

Serine/arginine splicing factor

Heterogeneous nuclear ribonucleoprotein

Glucose-6-phosphate dehydrogenase

RNA recognition motif

Arginine/serine dipeptide repeat domain

RNA immunoprecipitation

Crosslinking and immunoprecipitation

Multiplicity of infection

Adeno-associated virus

Sterol regulatory element-binding protein 1

Malic enzyme 1

Nuclear factor $k$-light chain enhancer of activated B cells

$\beta$-glucuronidase

Fatty acid synthase

Phosphoenolpyruvate carboxykinase

Stearoyle-CoA desaturase

False discovery rate

Inclusion level difference

RNA Integrity number 


\section{CHAPTER 1: LITERATURE REVIEW}

\section{RNA PROCESSING}

\section{1: Introduction}

For many years, students in Biology 101 courses around the country were taught the "Central Dogma," as it was proposed by Francis Crick: DNA makes RNA makes protein [1]. Even as Crick's dogma was being published in 1970, RNA was fast becoming more than just a messenger-pun intended-in the eyes of scientists everywhere. In particular, the discoveries during the late 1960s and early 1970s that mRNAs undergo extensive processing both during and after transcription began to chip away at the simplicity of the original dogma.

\section{2: Capping}

To ensure that transcribed mRNA survives long enough to be transported from the nucleus to the cytoplasm and translated into protein, the transcript must be protected from degradation by nucleases. During transcription initiation, eukaryotic RNA Polymerase II (Pol II) generates a primary RNA transcript from the template DNA strand. After the synthesis of about 25 nucleotides [2], the 5'-end of the mRNA is methylated. Known as "capping," the addition of a 7-methylguanine $\left(\mathrm{m}^{7} \mathrm{G}\right)$ at the $5^{\prime}$-end of the mRNA serves several important functions. First, the $m^{7} G$ cap helps to protect the newly-transcribed mRNA from degradation by rendering it resistant to $5 \rightarrow 3$ ' exonuclease activity [3]. Capping also enhances the processes of both RNA splicing (described later) and 3'-polyadenylation by defining the first exon of the primary transcript and interacting with polyA binding proteins, respectively [4]. 
Capping of the 5' end of the RNA is accomplished through a series of 3 enzymatic reactions. The first reaction involves an RNA triphosphatase that removes the $\gamma$-phosphate from the 5'-triphosphate of the first nucleotide of the transcript. A GTP-dependent RNA guanalyltransferase then transfers a guanine nucleotide to the mRNA, resulting in a 5'-5' triphosphate bridge and the loss of a pyrophosphate group. This guanine nucleotide, and the next 2 nucleotides are then methylated by guanine-7-methyltransferase. This enzyme transfers a methyl group from S-adenosylmethionine to the bases of the nucleotides. Importantly, addition of the $\mathrm{m}^{7} \mathrm{G}$ cap is indicative of RNA Pol II's transition from transcription initiation to elongation [2]. The hypophosphorylated C-terminal domain (CTD) of Pol II associates with both negative elongation factors and capping enzymes, which together cause Pol II to stall after the synthesis of about 25 nucleotides. This stalling allows capping to occur before Pol II can shift to the elongation phase of transcription. Once the cap is added, the capping proteins dissociate and positive elongation factors are recruited to restart Pol II and transition to the elongation phase of transcription [4].

After the addition of the $5^{\prime}-\mathrm{m}^{7} \mathrm{G}$ cap, the mRNA interacts with a heterodimeric protein complex consisting of a large subunit (CBP80) and a small subunit (CBP20) known as the cap binding complex (CBC). The $\mathrm{CBC}$ helps to stabilize the mRNA by protecting it from endonucleolytic cleavage and subsequent $5^{\prime} \rightarrow 3^{\prime}$ exonuclease activity and premature degradation of the transcript [3]. After the mRNA is fully transcribed and processed, the CBC helps to facilitate transport of the transcript from the nucleus to the cytoplasm through interactions with proteins at the nuclear pore complex. Once in the cytoplasm, the $\mathrm{CBC}$ is exchanged for eukaryotic initiation factor 4E (elF-4E), which promotes recruitment of additional translation initiation factors and interactions between ribosomal subunits and the nascent mRNA [5]. 


\section{3: Polyadenylation}

The final steps in RNA processing, cleavage and polyadenylation, take place at the 3'end of the transcript. 3'-end processing of mRNA is a 2-step process that begins when proteins associated with the Pol II CTD recognize a poly $(A)$ signal sequence in the transcript. This signal sequence, AAUAAA, is highly conserved and is located between 10 and 30 nucleotides upstream of the actual cleavage site [6]. The signal sequence is first bound by a protein complex, consisting of CTD-associated cleavage and polyadenylation specificity factor, cleavage factors I and II, and also poly(A) polymerase [7, 8]. Once this protein complex is formed, the mRNA is cleaved between the signal sequence and a downstream G/U-rich sequence. The exact mechanism by which cleavage occurs is unknown, but it is followed immediately by addition of $\sim 200$ adenosine residues by a CTD-associated templateindependent RNA polymerase known as poly(A) polymerase [9-11]. The polyadenylated mRNA is then bound by poly $(A)$ binding proteins, which interact with the cap binding complex at the $5^{\prime}$-end to protect the transcript from degradation by $3^{\prime} \rightarrow 5^{\prime}$ exonucleases [12]. As addition of the $\mathrm{m}^{7} \mathrm{G}$ cap at the $5^{\prime}$ end defines the first exon in an mRNA, the poly(A) tail defines the terminal exon at the 3 '-end.

\section{4: Splicing}

Splicing is a co-transcriptional RNA processing step that occurs in all eukaryotic cells where introns are removed and exons are joined together. Generally speaking, exons code for the amino acids that will ultimately be translated into protein, while introns are removed during splicing and thus do not contribute to the amino acid sequence of the gene.

A dynamic macromolecular complex of proteins and small nuclear ribonucleoprotein particles (snRNPs), collectively known as the spliceosome, is responsible for splicing 
catalysis in eukaryotes. The spliceosome assembles in a stepwise manner around specific sequence motifs that distinguish intronic versus exonic sequences. Through a series of protein-protein, protein-RNA, and RNA-RNA interactions, the spliceosome properly structures the mRNA to facilitate splicing catalysis [13-16]. Both exons and introns contain multiple cisacting sequence elements that are necessary for the recruitment and assembly of spliceosomal components at intron-exon boundaries (Figure 1a) [17].

The first two nucleotides of an intron are the 5' splice site (5'SS), while the last two nucleotides of the intron are the 3'-splice site (3'SS). The 5'SS is always GU, and the 3'SS is always AG. Approximately 25-50 nucleotides upstream of the 3'SS is a stretch of $15-20$ primarily pyrimidine nucleotides called the polypyrimidine tract. Further upstream of the polypyrimidine tract is a short nucleotide sequence called the branch sequence, within which lies an invariant adenosine nucleotide, called the branch point. This adenosine provides a critical substrate for the first step in splicing catalysis [14]. The 5' and 3' splice site sequences and the branch sequence are poorly conserved beyond the residues indicated [18].

The first step in spliceosome assembly is the formation of the early spliceosome, or "Ecomplex" (Figure 1b). U1 snRNP recognizes the 5'SS and associates with the mRNA via base-pairing. At the 3'-end of the intron, U2 accessory factor heterodimer (U2AF 65/35) recognizes the 3'SS and also interacts with the mRNA through base-pairing [19]. Next, U2 snRNP recognizes the branch sequence and interacts with U1 snRNP through an ATPdependent mechanism. This constitutes the formation of "A complex." It is important to note that metazoan introns are generally very large-about $5 \mathrm{~kb}$, on average $[17,20]$-while approximately $80 \%$ of exons are 200 bp or smaller, rarely exceeding 500 bp [20, 21]. The ability of the splicing machinery to recognize these relatively small exons amongst much larger introns is attributed to a process known as "exon definition." In this regard, the exons 
are said to be 'defined' by the exon-spanning interactions of U1 and U2 snRNPs that occur during early "A complex" formation [22]. Following a complicated series of rearrangements, U1 and U2 snRNPs subsequently form intron-spanning complexes [23]. The U1/U2 complex promotes folding of the mRNA such that the 5' and 3' ends of the intron move into close proximity in preparation for splicing catalysis.

Formation of "B complex" is characterized by the recruitment of the U4/5/6 tri-snRNP complex, which involves both RNA-RNA and protein-RNA interactions. At this point, another series of poorly-understood rearrangements and compositional changes take place, leading to "B complex" activation and subsequent dissociation of the U4 and U1 snRNPs. These rearrangements facilitate the first of two transesterification reactions. Still associated with the branch sequence, U2 snRNP contains a pseudouridine residue, which interacts with the branch point adenosine. This interaction causes the adenosine residue to bulge outward, exposing its 2'-OH group. The 2'-OH of the adenosine acts as a nucleophile and attacks the terminal phosphate on the exon at the 5' splice site (Figure 2a, top panel). The 5' end of the intron is released from the 3' end of the 5' exon, forming a lariat structure via 2',5' phosphodiester linkage with the branch point adenosine (Figure 2a, bottom panel). This constitutes " $\mathrm{C}$ complex" formation. Now, the 3'-OH on the 5' exon is exposed and the lariat remains attached to the 3' exon.

Finally, "C complex" completes the splicing process by catalyzing the second transesterification reaction. In this step, the exposed 3'-OH on the 5' exon acts as the nucleophile and attacks the terminal phosphate on the 3 ' end of the intron (Figure 2b, top panel). The end result is the excision of the lariat intron and ligation of the 5' and 3' exons (Figure 2b, bottom panel). The spliceosomal components then dissociate, the intron is degraded by nucleases and the snRNPs are recycled for future splicing reactions [24]. 


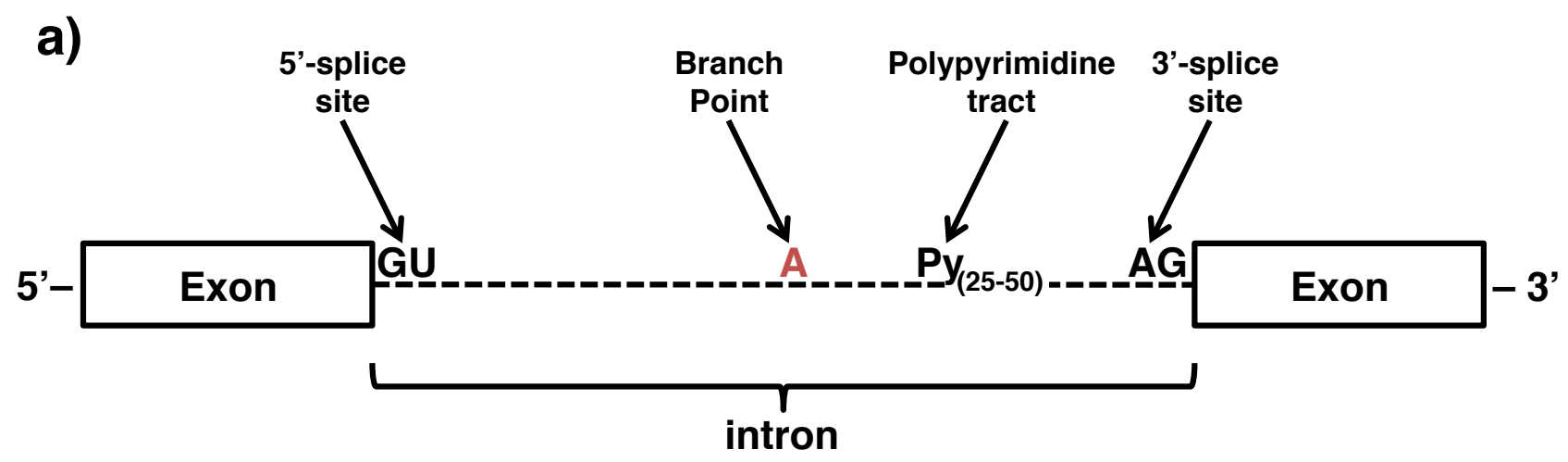

b)

E Complex

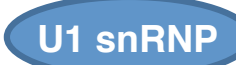

\section{U2AF 65/35}

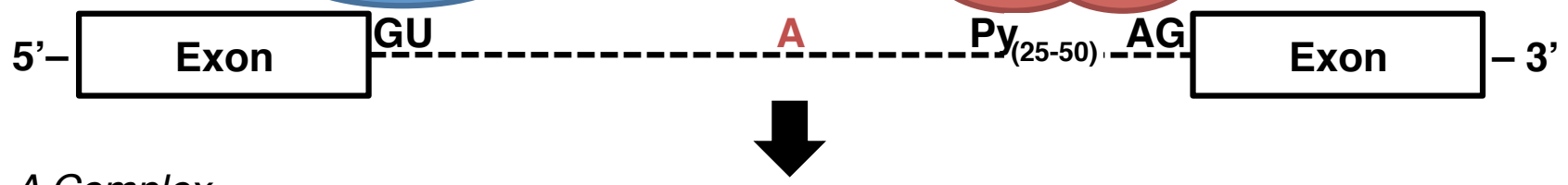

A Complex

\section{U1 SnRNP}

5'- Exon GU - -

\section{U2AF $65 / 35$}

- $P y_{(25-50)} A G$ Exon

B Complex
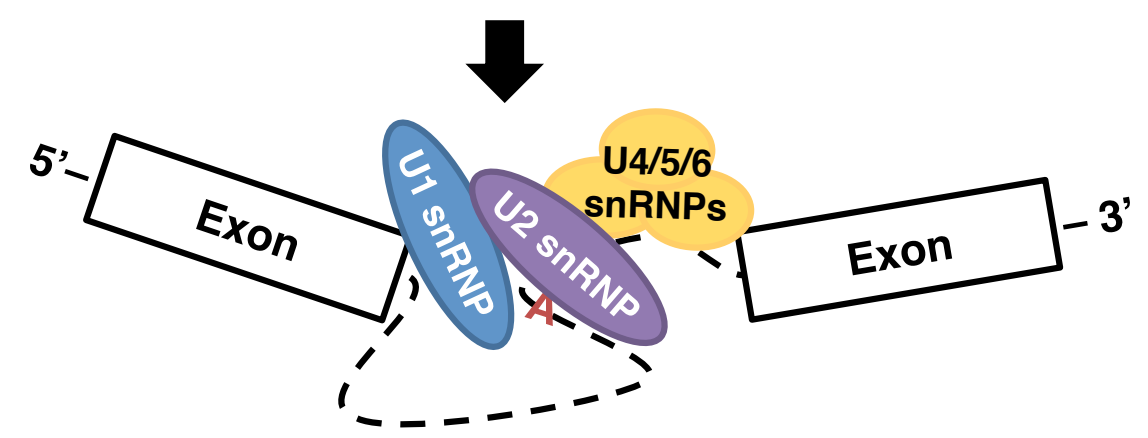

C Complex

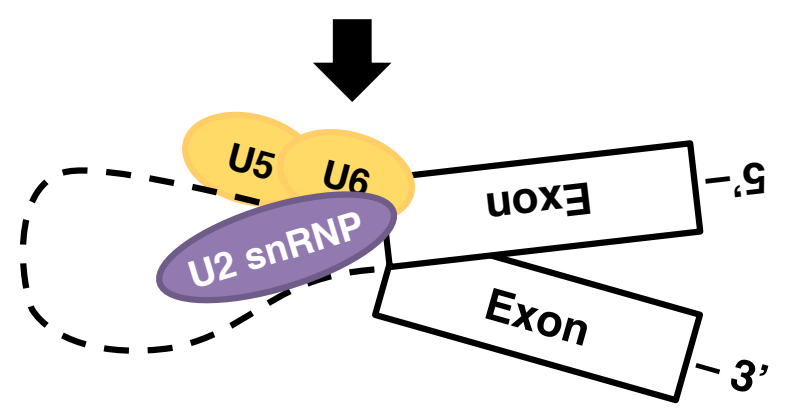

Figure 1: mRNA and the spliceosome

a) Diagram of typical pre-mRNA structure. b) Schematic of spliceosome complex formation 

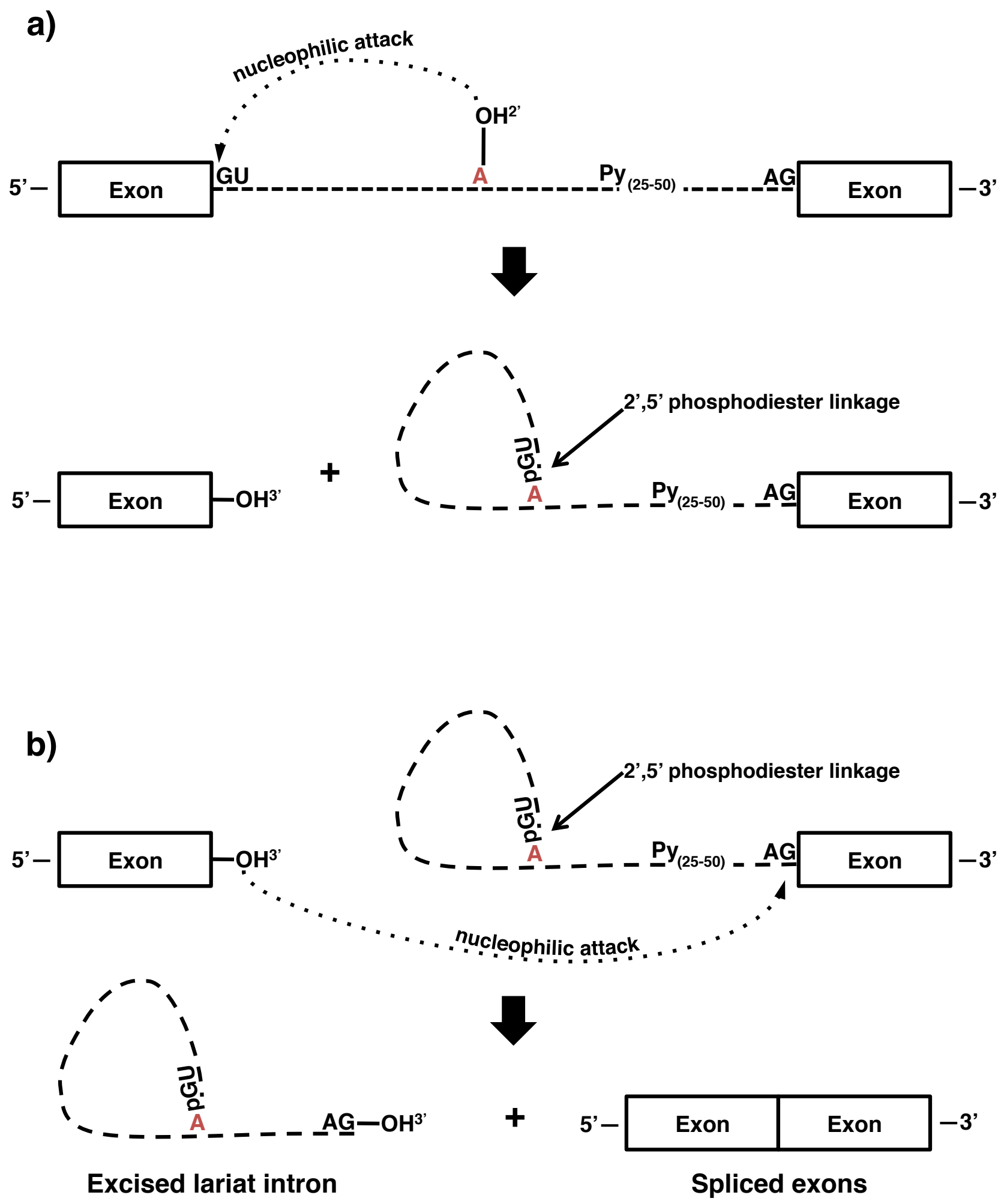

Figure 2: Splicing catalysis

a) First transesterification reaction. b) Second transesterification reaction. 


\section{5: Post-Splicing}

It is worthwhile to note that even though spliceosomal proteins and snRNPs dissociate after splicing, mRNA is always associated with cellular proteins. Proteins that are associated with mature (spliced) mRNAs are involved in a variety of functions, such as aiding in export of the mRNA from the nucleus to the cytosol, and mRNA quality control. For example, as a direct consequence of completed splicing, a multi-protein complex called the exon junction complex (EJC) is deposited approximately $20 \mathrm{nt}$ upstream of exon boundaries, as well as in the 3' untranslated region (UTR) of the transcript [25]. EJC-associated proteins interact with the mRNA in a largely sequence-independent manner [26]. In the nucleus, the primary function of the EJC is to aid the export of processed mRNAs to the cytosol for translation via interactions with components of the nuclear pore [27]. In the cytosol, EJCs remain stably associated with the mRNA where they enhance translation by marking exon-exon boundaries and also become an important component of mRNA quality control [28, 29].

Once such quality control process that is heavily reliant on EJCs is called nonsensemediated decay (NMD). As is often the case with many cellular processes, errors can occur at any number of points along the way. In the case of mRNA splicing, NMD represents a surveillance pathway that can prevent improperly and/or incompletely spliced transcripts from being translated into protein [30]. For example, if the GU making up the conventional 5'SS of an intron is mutated to a GG, the intron may not be removed because the spliceosome does not recognize the mutated 5'SS. This phenomenon, termed intron retention, is a form of alternative splicing and will be discussed in-depth in later sections. If the retained (unspliced) intron contains an in-frame stop codon, the transcript is said to contain a nonsense mutation. In the cytosol, translation stops at the erroneously-introduced stop codon, also known as a premature termination codon. If an EJC is deposited between 50-55 nucleotides upstream of 
the premature termination codon, the transcript is targeted for NMD [31-33]. In NMD, EJCassociated factors Upf2 and Upf3 interact with the cytosolic NMD effector Upf1 to activate the NMD pathway [30]. Other EJC-associated proteins then coordinate a series of events that ultimately destabilize the improperly spliced transcript, thus preventing translation. In this regard, NMD can be viewed as a regulatory mechanism that guards against the accumulation of non-functional and/or potentially toxic proteins that may occur due to errant splicing or as a form of control over gene expression [26, 29, 34].

\section{6: Additional Splicing Regulatory Sequences}

The 5'SS, 3'SS, polypyrimidine tract, and branch sequences are indispensable to the splicing process, but these four "core" elements are not sufficient to promote an accurate splicing program on their own [35]. Additional cis-acting sequence elements exist that recruit trans-acting splicing factors that can enhance and/or inhibit splicing in a context-specific manner. Exonic splicing enhancers (ESEs) and intronic splicing enhancers (ISEs) are sequence elements located within exons and introns, respectively, that are bound by proteins that function as splicing enhancers, such as members of the serine/arginine-rich (SR) protein family [36]. By binding to ESE/ISE elements, SR proteins can exert their influence by helping to recruit proteins and/or snRNPs during assembly of the spliceosome. Vis-à-vis, exonic splicing silencers (ESSs) and intronic splicing silencers (ISSs) are sequence elements that are bound by proteins that act as splicing inhibitors, such as members of the heterogeneous nuclear ribonucleoprotein (hnRNP) family [37]. Generally acting in opposition to SR proteins, hnRNPs bound to ESS/ISS elements can inhibit splicing by blocking the recruitment of spliceosomal components. Both SR proteins and hnRNPs and their respective roles in 
enhancement and inhibition of splicing will be discussed in greater detail in the following sections.

The role that a particular splicing regulatory element plays in splicing is heavily dependent on the context in which it is found, and on which trans-acting factors are bound to it. Of note, there is no "true" consensus sequence. They vary widely in both sequence and length and must therefore be characterized individually to determine whether it functions as a splicing enhancer or inhibitor [38]. For example, a guanine-rich regulatory element that is located within an intron usually behaves like an ISE, because it attracts and binds a certain splicing factor. However, a guanine-rich regulatory element that is located within an exon often functions as an ESS because it attracts that same splicing factor (discussed further in section 3.2) [39, 40]. Finally, splicing regulatory sequences are key regulators of both constitutive and alternative splicing [41].

\section{TYPES OF SPLICING}

\section{1: Introduction}

The importance of mRNA splicing lies in the fact that it does not occur uniformly in every transcript every time. In any given mRNA, some exons are constitutively spliced while others may be alternatively spliced. Constitutive exons are present in all versions of a particular transcript, whereas alternative or "cassette" exons are those that appear only under certain cell/tissue-specific conditions (Figure 3a) $[42,43]$. The manner in which a particular transcript is ultimately spliced is dependent on a variety of factors, including the presence and/or absence of cis-acting sequence elements and trans-acting factors. 
The term 'alternative splicing' is a blanket phrase used to describe splicing events that yield transcripts that differ from those that would be expected based on the sequence. This means that a single multi-exon mRNA has the potential to give rise to many different protein isoforms depending on the manner in which it is spliced [22]. As recently as 2002, alternative splicing was estimated to occur in $40-60 \%$ of human genes [44]. Presently, it is thought that more than $95 \%$ of human genes are alternatively spliced in some capacity; thus, alternative splicing has become the rule rather than the exception-emerging as a major source of proteomic diversity [45, 46]. Most alternative splicing events can be grouped into one of four of general categories: alternative 5'SS choice (Figure 3b), alternative 3'SS choice (Figure 3c), cassette exon skipping/inclusion (Figure 3d), and intron retention (Figure 3e).

\section{2: Alternative 5' or 3' Splice Site Choice}

Alternative 5'SS choice is a form of alternative splicing that occurs when U1 snRNP recognizes more than one 5'GU codon as the 5'SS. As a result, an exon may be lengthened or shortened depending on the locations of the alternative 5'GU codons. For instance, if U1 snRNP recognizes and binds to a 5'SS that is located farther downstream than the canonical 5'SS, the nucleotides between the original and the alternative sites would then be included as part of the exon, lengthening the exonic sequence (Figure 3b, upper panel). If the alternative 5'SS were located upstream of the usual 5'SS, the exonic sequence would be truncated (Figure 3b, lower panel). Alternative 3'SS choice is analogous to alternative 5'SS selection, except that recognition of the alternative 3' splice sites is by U2 snRNP (Figure 3c).

Utilization of alternative splice sites can sometimes produce proteins with completely different functions. One example of the effects alternative 5'SS selection can have on the proteins that are eventually expressed is the $B c l-x$ gene. The transcript of the $B c l-x$ gene 


\section{a) "Normal" Condition}

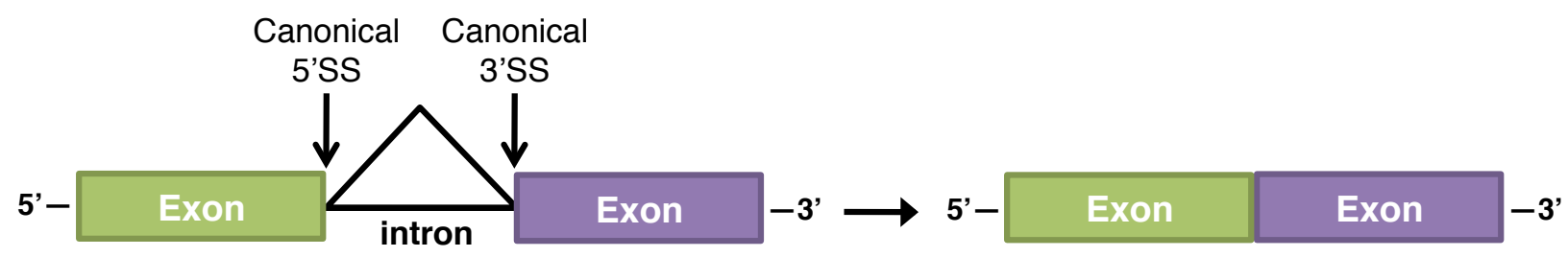

b) Alternative 5' splice site choice

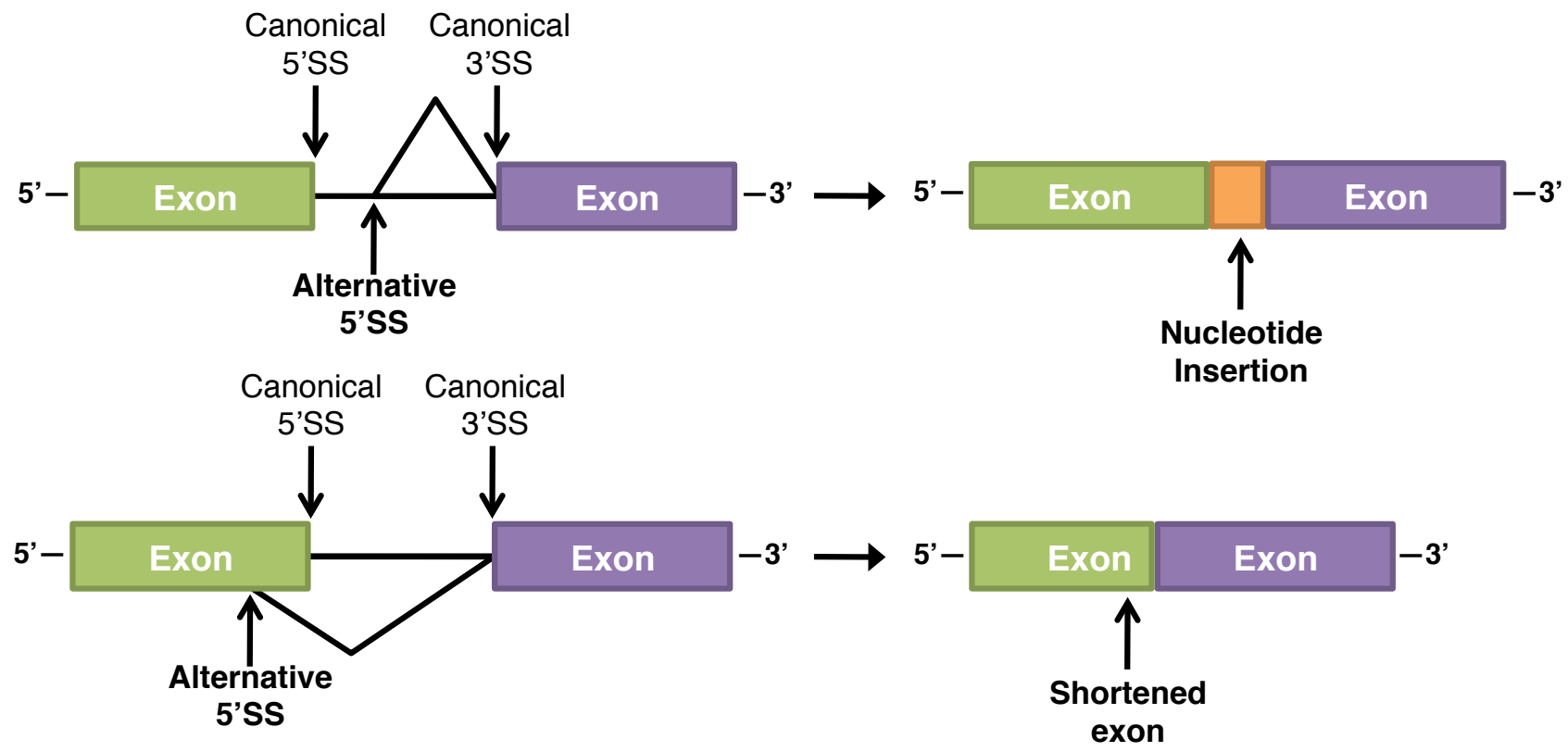

Figure 3: Types of Alternative Splicing

Alternative splicing events generally fall into one of four categories depicted above. Colored boxes indicate coding sequences and black lines depict introns. a) "Normal" splicing reaction in which the spliceosome recognizes both the 5'SS and the 3'SS, excises the intron, and joins the flanking exons. b) Alternative 5'SS choice, in which the spliceosome recognizes a downstream 5'SS (upper), introducing additional coding nucleotides at the 3' end of the 5' exon, or an upstream 5'SS (lower) that results in a truncated 5' exon. 
c) Alternative 3' splice site choice

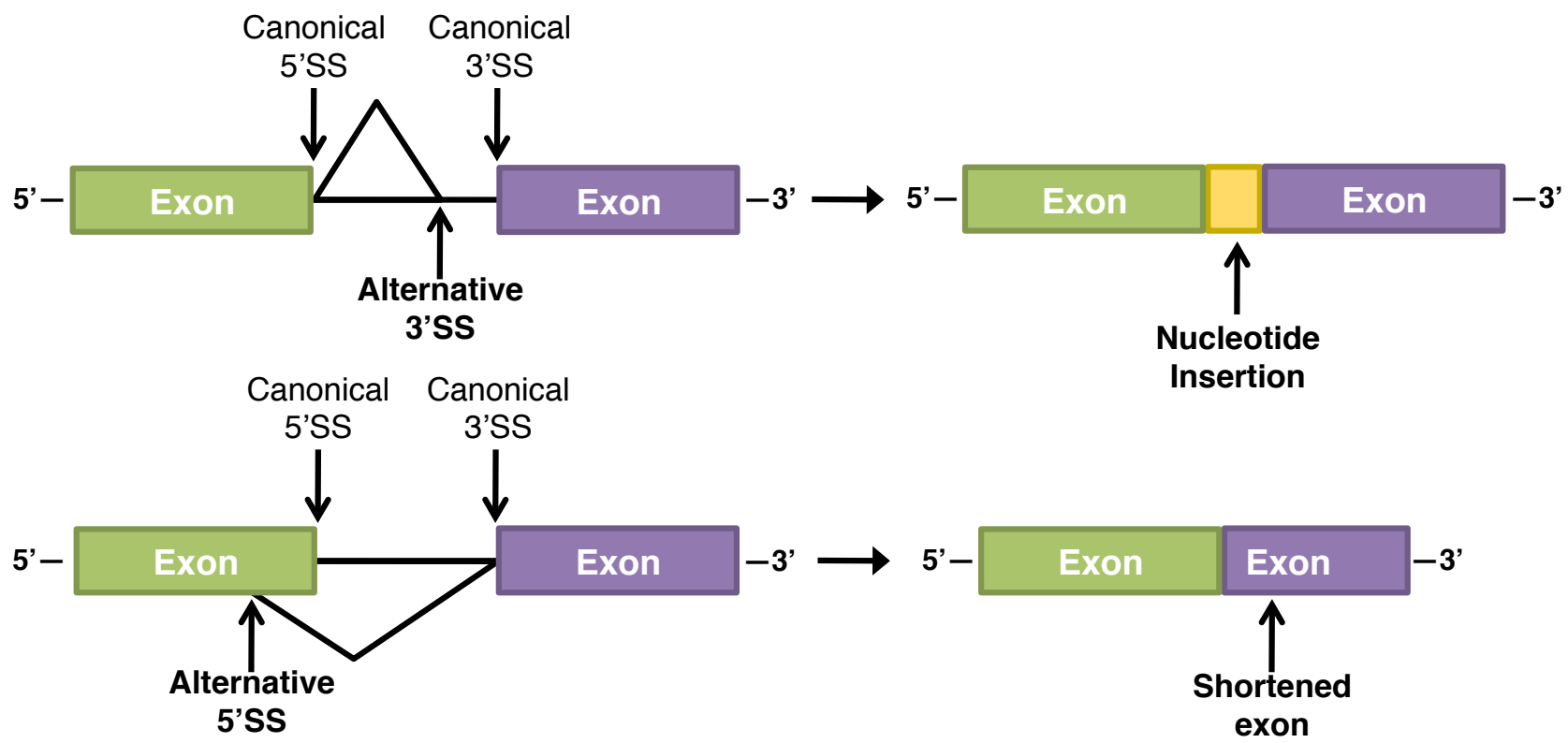

d) Alternative skipping / inclusion of exons

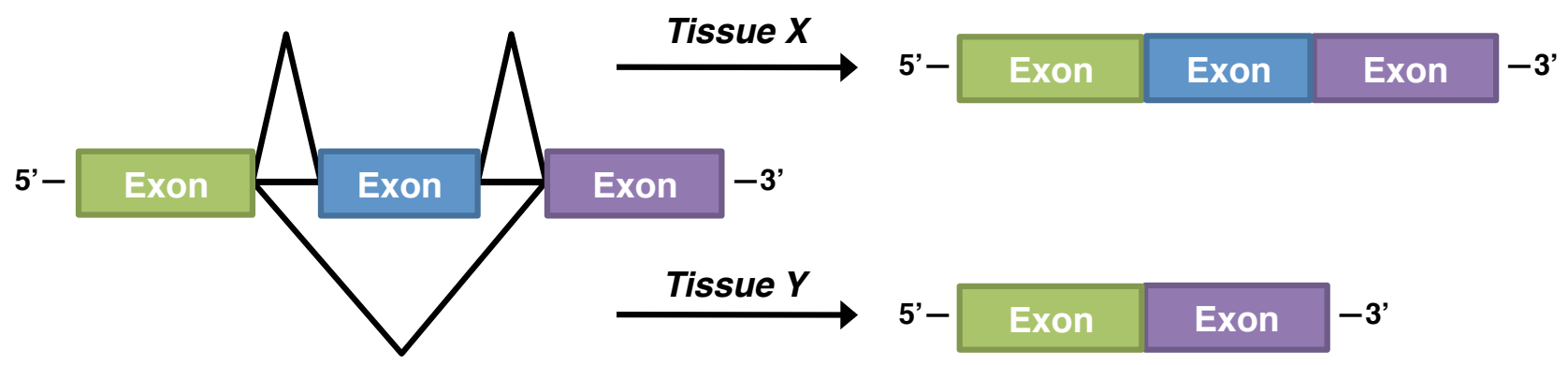

e) Intron retention

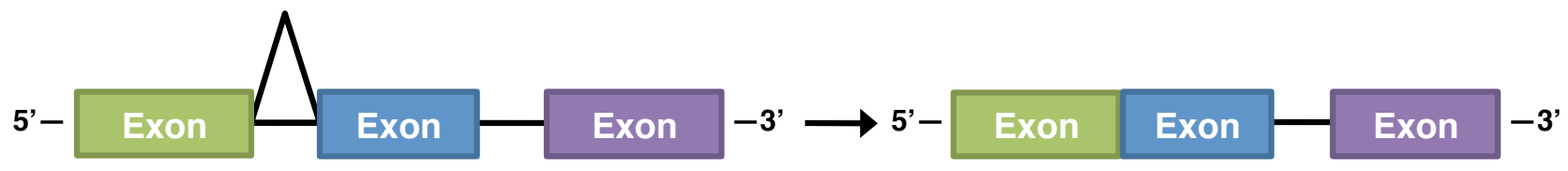

c) Alternative 3'SS choice, in which the spliceosome recognizes an upstream 3'SS (upper), resulting in addition of coding nucleotides at the 5' end of the 3' exon, or an upstream 5'SS (lower) that results in a truncated 3' exon. d) Alternative skipping/inclusion of exons can occur when a certain exon is only included under certain conditions. The blue exon is properly spliced and included in the final mRNA transcript in tissue $X$ (upper), but the exon is not included in tissue $Y$ (lower). e) Intron retention happens when the spliceosome fails to excise an intron for some reason, allowing the intron to stay in the final transcript. 
contains two 5' splice sites that change the length of the second exon [47]. Use of the distal 5'SS produces a transcript that codes for $B c l-x S$, while use of the proximal 5'SS increases the length of exon 2 by $189 \mathrm{nt}$ and codes for a protein called $B c /-x L$. In this regard, choice of 5'SS determines which of two functionally distinct proteins are eventually produced: either the proapoptotic $B c l-x S$ or the anti-apoptotic $B C l-x L$. The decision to utilize one splice site versus another is dependent on a combination of several cell-, tissue-, and environmentally-specific factors. Again, the complexity of regulation is exemplified by the case of $\mathrm{BCl}-\mathrm{x}$. Alternative splicing of $B c l-X$ is tightly regulated by cell-cycle progression [48], which by extension is dependent on the cell type/tissue in which it is to be expressed, which in turn determines the expression profiles of the splicing factors present as well as the signaling pathways that govern their activities $[49,50]$.

Another example of alternative splicing by alternative splice site utilization is caused by a genetic mutation and occurs in a gene called $B B S 8 . B B S 8$ is a core component of a large protein complex called the BBSome, which is involved protein trafficking in ciliated cells throughout the body [51]. In 2010, a mutation was discovered in which the 3'SS of BBS8 exon $2 a$ is changed from $A G$ to $G G$ [52]. It is important to note here that $B B S 8$ is ubiquitously expressed, but transcripts containing exon 2a are found exclusively in retinal photoreceptor cells [52]. In photoreceptor cells, the AG>GG mutation at the BBS8 exon 2a 3'SS forces the splicing machinery to use an alternative 3'SS that is located $7 \mathrm{nt}$ downstream [53]. The clinical manifestation of alternative 3'SS usage here is a photoreceptor-specific defect called retinitis pigmentosa, which eventually leads to photoreceptor death and loss of vision. The authors of a 2014 study found that the use of this alternate 3'SS causes a truncated exon 2a to be included in the final transcript, which they hypothesize introduces a premature termination codon and subsequently, loss of the BBS8 protein [53]. 
The frequency of alternative splicing events in higher eukaryotes that fall into the alternative 5' and 3' splice site selection categories is around $8 \%$ and $18 \%$, respectively [54]. Several factors can influence the recognition of alternative 5' or 3' splice sites, including the length of the surrounding exons, presence or absence of splicing regulatory elements, and presence or absence of certain trans-acting splicing proteins and snRNPs [55-58].

\section{3: Cassette Exon Skipping/Inclusion}

The most common form of alternative splicing, cassette exon skipping/inclusion is thought to account for around $40 \%$ of alternative splicing events in higher eukaryotes [54]. In this type of splicing, a cassette exon is included in some situations while it is excluded in others (Figure 3d). Like other forms of alternative splicing, inclusion or exclusion of particular cassettes exons is dependent on the combinatorial effects of several regulatory factors in the context in which the exons are found. Using the previously-discussed example of $B B S 8$ alternative splicing, transcripts containing exon $2 a$ are exclusive to photoreceptors, while exon $2 \mathrm{a}$ is skipped in all other cell types [53]. As such, BBS8 exon 2a can be classified as a cassette exon because it is present in one cell type but not others. This is an example of how alternative splicing of a cassette exon can allow a single mRNA to code for multiple proteins with tissue-specific functions.

The cardiac troponin-t $(\operatorname{Tn} T)$ gene is an example of alternative splicing that is regulated developmentally and by development-specific factors. The default splicing program in adult cardiomyocytes is to skip exon 5 , with more than $95 \%$ of $T n T$ mRNA missing the exon. In embryonic hearts, however, more than $90 \%$ of $T n T$ mRNAs include exon 5 in the final transcript [59]. The result of this alternative splicing event is that embryonic cardiac $\operatorname{TnT}$

proteins include a 10-amino acid region that is absent from the adult $T n T$ protein [60]. The 
protein produced from the mRNA including exon 5 confers increased calcium sensitivity in developing cardiac myofibrils, thereby increasing ATPase activity and aiding in their maturation [61]. Often, there are multiple instances of cassette exon skipping/inclusion within a single multi-exon transcript, which can confer certain properties to the protein depending on the combination of exons that are included in the fully-processed mRNA. Furthermore, the decision to include certain cassette exons and exclude others is, like other forms of alternative splicing, heavily reliant on the presence of specific splicing regulatory factors that are present in the developmental and/or tissue-specific context in which the mRNA is to be expressed.

\section{4: Intron Retention}

Recent studies estimate that intron retention accounts for approximately $45-50 \%$ of alternative splicing events in higher eukaryotes [62], yet it remains one of the least understood forms of alternative splicing [54, 62]. As the name implies, this type of splicing occurs when an intron is not excised from the pre-mRNA and is retained in the mature transcript (Figure 3e). As discussed briefly in section 1.5 above, retained introns frequently introduce premature termination codons into the processed mRNA that target the transcript for degradation by the NMD surveillance pathway [63-65]. Traditionally viewed mainly as a consequence of aberrant splicing, recent studies have provided evidence that intron retention may actually represent a regulated, pre-translational mechanism of control over gene expression [65-67].

Work in our laboratory has been focused on understanding the role of intron retention in the regulation of hepatic gene expression [67-70]. Expression of glucose-6-phosphate dehydrogenase (G6PD), an integral enzyme involved in de novo lipogenesis and protection 
from oxidative stress, is regulated by changes in nutrient availability in liver [71]. During starvation, abundance of G6PD mRNA is decreased by more than $70 \%$, while refeeding starved animals for $24 \mathrm{~h}$ causes a 15-fold increase in abundance of G6PD mRNA [72]. Additional experiments showed that the these nutrient-induced fluctuations in G6PD mRNA accumulation were the result of changes in the rate of splicing of the primary transcript [69, 72, 73]. Specifically, a splicing regulatory element located within exon 12 was found to be responsible for mediating the effects of nutrients on the splicing of G6PD mRNA [67, 70, 74, 75]. Using in vitro splicing assays, Szeszel-Fedorowicz et. al. found that spliceosome assembly (and thus, splicing itself) was inhibited by the presence of exon 12, even in in vitro splicing substrates where exon 12 was substituted for other readily-spliced exons elsewhere in the transcript [67]. Furthermore, the same study demonstrated that several hnRNPs and SR proteins (splicing silencers and splicing enhancers, respectively) bound to exon 12 . These results suggested the presence of both ESS and ESE elements within exon 12 that influences splicing efficiency via differential binding of splicing enhancer and splicing silencer proteins [67]. Importantly, these findings suggested that intron retention may be an important mechanism of regulation of metabolic gene expression in the liver, and served as a major turning point in the direction of our laboratory's research.

\section{REGULATION OF SPLICING}

\section{1: Introduction}

There are several proteins that exert control over the splicing process in addition to the "core" proteins and snRNPs of the spliceosome. The two main families of trans-acting splicing factors are the hnRNPs and the SR proteins [22]. In general, SR proteins function as splicing 
enhancers, while hnRNPs are thought to act as splicing silencers $[76,77]$. These proteins exert their functions in ways that are dependent on both the tissue- and developmentallyspecific contexts in which they are expressed [78-87]. Extracellular signaling molecules, such as growth factors, hormones, and nutrients, can affect the splicing process by altering the expression, activity and/or intracellular location of both hnRNPs and SR proteins [88-92]. Since their initial discovery, a diverse array of hnRNP and SR protein functions have been described, including roles in chromatin remodeling and transcription, mRNA splicing, nuclear export, mRNA stability, and translation [92-97]. SR proteins and hnRNPs are ubiquitously expressed and highly conserved; thus supporting an essential role of these splicing regulatory proteins in eukaryotic cells $[90,93,98]$.

\section{2: hnRNPs}

The hnRNP family of RNA-binding proteins is composed of more than 20 structurallydistinct members [77]. Often described as "splicing silencers," more than half of all hnRNPs are known to negatively impact the recognition of splice sites by the core splicing proteins and snRNPs [99]. This silencing can occur either by physically masking splice sites and/or directly antagonizing the binding of splicing enhancer proteins [22, 74].

The domain structure of the individual hnRNPs is diverse. Every hnRNP contains one or more RNA recognition motifs (RRMs) or K Homology $(\mathrm{KH})$ domains, through which the protein interacts with target sequences [77]. Some hnRNPs contain additional auxiliary domains, including Arg-Gly-Gly tripeptide repeat boxes, Ser-Arg-Gly-Tyr domains, prolinerich, and glycine-rich domains [100]. Finally, some hnRNPs also contain nuclear export signals [101], nuclear retention signals [102], and nuclear shuttling domains [103]. 
It is important to note that hnRNPs do not function exclusively as splicing silencers. As discussed in section 1.6, the location of their binding sites (intronic vs. exonic) is sometimes the determining factor in this regard. In that example, hnRNP $\mathrm{H}$ has a dual function as a splicing silencer and a splicing enhancer, depending whether the target sequence-a guanine-rich element-is located within an exon or an intron, respectively [39, 40].

More than half of the known hnRNP family members have documented functions in RNA splicing. Our laboratory has focused particularly on hnRNP $\mathrm{K}$ and its role in regulating the splicing of G6PD. As discussed in section 2.4, exon 12 of the G6PD mRNA transcript contains cis-acting sequences that are bound by different splicing proteins that ultimately affect the efficiency of exon 12 splicing [67]. One of these splicing regulatory elements functions as an ESS, and that ESS can be bound by hnRNPs K, L, and/or A2/B1 $[67,74,75]$. Since the abundance of spliced G6PD mRNA in liver is significantly decreased during starvation [69], we hypothesized that the binding of these hnRNPs may be regulated by nutrient status. Indeed, of the three hnRNPs identified, expression and binding of only hnRNP $\mathrm{K}$ was affected [74]. Specifically, we observed a 3-fold increase in hnRNP K protein expression in the livers of starved mice versus mice that were starved for $18 \mathrm{~h}$ and refed for $18 \mathrm{~h}$, while there was no significant change in expression of hnRNP L or A2/B1 [74]. Furthermore, through the use of both overexpression and knockdown approaches, and the use of a technique known as RNA Immunoprecipitation (RIP), we determined that binding of hnRNP K occurs in intact cells and that its binding is regulated by starvation [74]. In starved livers, hnRNP K binding to G6PD exon 12 is increased 12.5-fold relative to refed livers, which was accompanied by a $60 \%$ decrease in the abundance of G6PD mRNA [74]. These studies were the first demonstration that the expression and splicing function of an hnRNP is regulated by nutrient status. 


\section{3: SR Proteins}

Where hnRNPs are generally classified as splicing silencers, the serine/arginine rich (SR) proteins are a family of splicing factors that generally function as enhancers of splicing [93]. The canonical SR protein family members include SRSFs 1-12, previously known as SF2/ASF, SC35, SRp20, SRp75, SRp40, SRp55, 9G8, SRp46, SRp30c, SRp38, p54, and SRrp35, respectively (Figure 4) [86, 104]. SR proteins are predominantly located in the nucleus and are generally concentrated within subnuclear compartments called speckles [105]. Structurally, SR proteins contain one or two RNA recognition motifs (RRM) at the Nterminus and a C-terminal arginine/serine (RS) dipeptide repeat domain [93].

The N-terminal RRMs are critically important for splice site selection and substrate specificity of SR proteins $[89,106]$, and can also influence nucleocytoplasmic localization [107]. The RRM interacts with nascent mRNA transcripts directly by binding specific sequences within exons and introns. The cis-acting regulatory sequences to which SR proteins bind are highly degenerate, but optimal binding sequences have been described for a few SR proteins using SELEX, a computational technique, and in vitro experiments such as crosslinking and immunoprecipitation (CLIP) [108, 109]. The latter identifies sequences that are more likely to be physiologically relevant.

Five of the SR proteins (SRSF1, SRSF4, SRSF5, SRSF6 and SRSF9) contain two RRMs while the remaining seven (SRSF2, SRSF3, SRSF7, SRSF8, SRSF10, SRSF11 and SRSF12) have only one (Figure 4). In SR proteins containing two RRMs, the second RRM is sometimes referred to as a 'RRM homology domain' because it generally does not match the binding consensus sequence and is not thought to contact the RNA directly [90]. This is not to say that the second RRM is dispensable-on the contrary; 5' splice site selection in adenoviral E1A pre-mRNA was altered in cells expressing a mutant SRSF1 protein lacking its 
second RRM [89]. In addition to direct binding to target mRNAs, RRMs are involved in the regulation of SR protein distribution within the cell. For example, arginine methylation in the RRM of SRSF2 is critical for nuclear localization. Blocking this methylation results in aberrant accumulation of SRSF2 in the cytoplasm where it is unable to participate in splicing of target mRNAs [107].

The RS domain lies downstream of the RRM(s) with serine/arginine dipeptide repeats accounting for more than $40 \%$ of the amino acid residues in the C-terminal region [104]. This domain acts as a nuclear localization signal by interacting specifically with transportin-SR, a member of the $\beta$-karyopherin family which mediates the nuclear import of SR proteins [86].

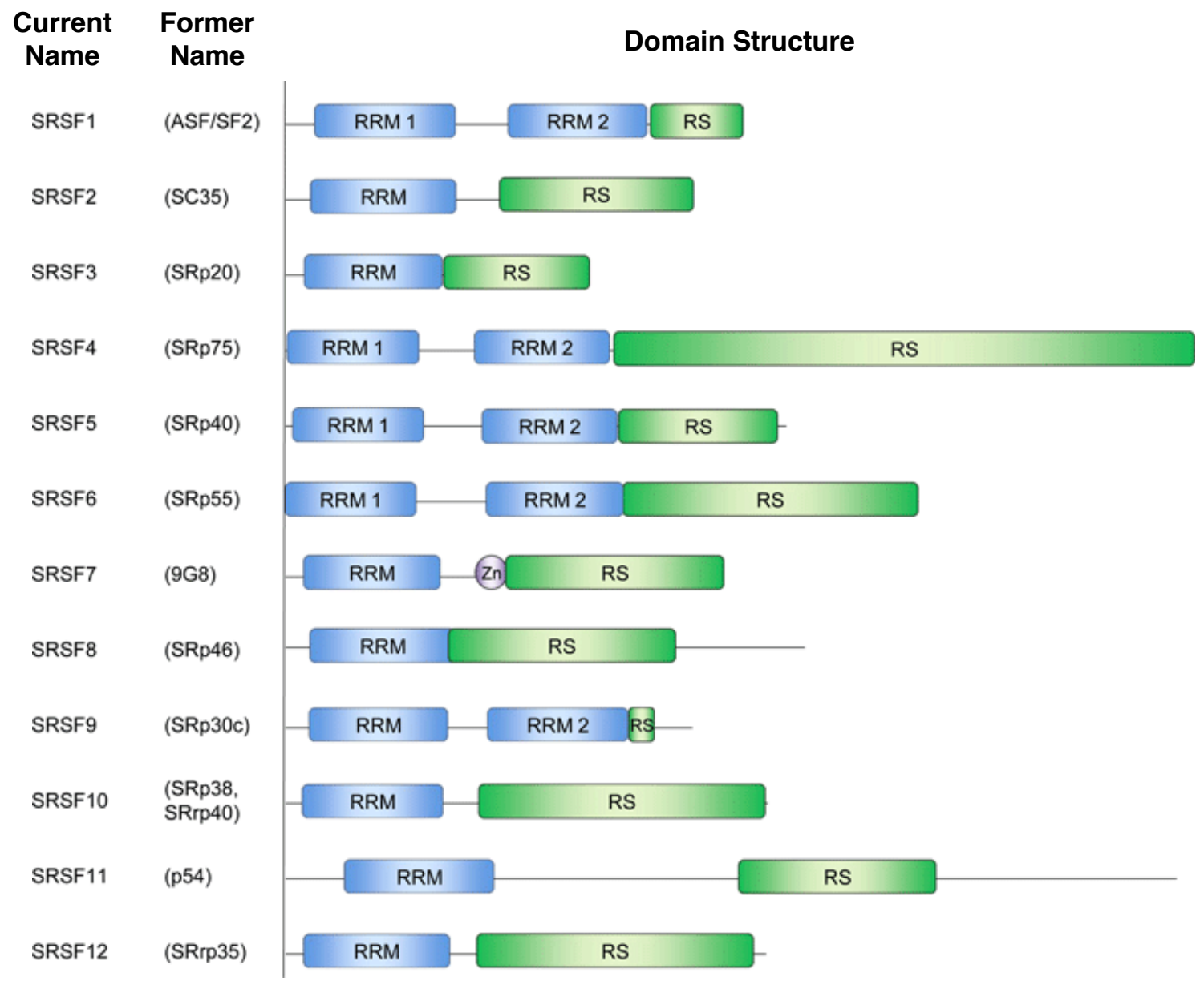

Figure 4: The SR protein family

Name and domain structures of the 12 SR proteins. Figure adapted from Twyffels et. al., 2011. $R R M$ : RNA recognition motif, $R S$ : arginine/serine dipeptide repeat domain, Zn: Zinc-finger domain. 
RS domains can be phosphorylated on serine residues by a several protein kinases, including SR protein kinases and cdc2- like kinases [110]. Phosphorylation of the RS domain has been shown to affect the splicing activity, amount, and distribution of SR proteins within the nucleus and/or the cell $[85,86,89,91,110-118]$.

\section{4: Regulation of SR proteins}

The most well-characterized mechanism of SR protein regulation is the reversible post-translational serine phosphorylation within the RS domain. Phosphorylation of the RS domain is known to be a key regulator of SR protein function at nearly every stage of splicing by altering the binding properties of SR proteins to both spliceosomal components as well as target mRNA transcripts $[119,120]$. In order for SR proteins to be released from speckles and allowed to travel to sites of active splicing, their RS domains must be phosphorylated [91, 121]. The converse is true as well-in order for SR proteins to return to speckles following completed splicing, they must be dephosphorylated [122]. A handful of kinases have been shown to directly phosphorylate SR proteins in the nucleus, including the cdc2-like kinases, topoisomerase I, and Akt [91, 123, 124], while dephosphorylation is known to be facilitated by protein phosphatase 1 (PP1) [122].

The phosphorylation state of SR proteins is also known to affect their ability to interact with spliceosomal proteins in both early and later events in spliceosome assembly and splicing catalysis $[119,125]$. Some studies suggest that SR proteins increase the efficiency of splicing by acting as molecular 'bridges' to enhance the juxtaposition of the 5' and 3' splice sites for lariat formation $[126,127]$. It has also been proposed that SR proteins become dephosphorylated prior to splicing catalysis, thereby weakening protein-protein interactions with snRNPs and possibly weakening the interaction between the U1 snRNP and the 5' splice 
site to allow the splicing reaction to proceed $[119,128]$. Phosphorylation of SRSF1 has been shown to enhance its interaction with the U1 snRNP during the initial stages of spliceosome assembly; thereby facilitating the recruitment of U1 to the 5' splice site [116]. Interestingly, in vitro splicing assays performed in the presence of a protein phosphatase showed that while spliceosomes continued to form, SR proteins were not incorporated and the splicing reaction did not proceed to the first catalytic step [125]. This study suggests that dephosphorylation of SR proteins following spliceosome assembly is required for the splicing reaction to proceed.

In addition to changes in nuclear localization, the phosphorylation status of some SR proteins is known to affect nucleocytoplasmic localization as well. SRSF1, SRSF3, SRSF4, SRSF6, SRSF7 and SRSF10 are known to shuttle between the nucleus and the cytoplasm $[89,129,130]$. , it was thought that the dephosphorylation that occurs in the RS domain toward the end of the splicing was also required for SR protein nuclear export. However, in studies where the RS domain from SRSF1 was fused to a reporter protein, it was found that the RS domain alone was not sufficient to promote nuclear export of the reporter protein [129]. Shuttling SR proteins also interact with the TAP/NFX1 receptor to aid in mRNA export, however; hypophosphorylated SR proteins cannot bind TAP [131]. This study noted that the interaction of RS domain with TAP was heavily dependent on the arginine residues in that domain and that serine phosphorylation probably blocked this interaction. It is possible that negatively charged phosphoserine residues would interact with the positively charged arginine residues to block their interaction with TAP [130] and preventing the SR protein from aiding mRNA export.

In the cytoplasm, phosphorylation of the RS domain was found to facilitate the interaction of SR proteins with transportin-SR, thus allowing for their nuclear import via nuclear pore complexes following translation [115]. The SR protein-specific kinases SRPK1 
and SRPK2 are distributed throughout both the nucleus and the cytoplasm and directly phosphorylate cytoplasmic SR proteins $[114,132]$. In addition to facilitating the interaction of SR proteins with nuclear import machinery, cytoplasmic phosphorylation has also been shown to affect their function in translation initiation and elongation [116]. For example, in sucrose gradient sedimentation of cytosolic extracts, dephosphorylated SRSF1 was found in polysome-containing fractions while hyperphosphorylated SRSF1 was not-suggesting that phosphorylation status is also critical for non-splicing cytoplasmic functions of SR proteins $[116,130]$

\section{5: SRSF3 \& Regulation of G6PD mRNA Splicing}

SRSF3 is the smallest of the 12 SR proteins, at 164 amino acids. It contains one $\mathrm{N}$ terminal RNA recognition motif (RRM) and an arginine-serine (RS) dipeptide repeat domain spanning approximately 60 amino acids at the C-terminus. SRSF3 is one of the SR proteins that is known to shuttle between the nucleus and the cytoplasm to participate in non-splicing functions, including mRNA transport and cap-dependent translation [86]. In mouse models, SRSF3 knockout embryos die prior to blastocyst formation, thus, while structurally similar to other SR proteins, SRSF3 is essential and functionally non-redundant [133]. Furthermore, conditional knockout of SRSF3 in mouse liver interferes with hepatocyte development and negatively affects hepatic lipogenic and cholesterol biosynthetic pathways [82]. SRSF3 is also known to play critical roles in the production of viral particles from several families, including picoronaviridae, retroviridae, adenoviridae, papillomaviridae, and herpesviridae [134-139].

The specific cellular functions of SRSF3 are not as extensively characterized as some other SR proteins, with only a handful of regulatory targets having been identified in vitro-its own transcript included [140-143]. SRSF3 expression is known to be tightly controlled by an 
auto-regulatory mechanism $[143,144]$. Increased SRSF3 protein expression promotes the inclusion of exon 4 in the SRSF3 mRNA transcript. SRSF3 exon 4 is known as a "poison exon" because inclusion of this exon creates an in-frame premature termination codon in the transcript, ultimately decreasing the amount of functional SRSF3 protein in the cell [143].

The central results guiding our research have been the observations that splicing of the G6PD mRNA is regulated by the nutritional status of the animal [71]. As the rate-limiting enzyme in the pentose-phosphate pathway, G6PD is responsible for the production of reducing equivalents in the form of $\mathrm{NADPH}+\mathrm{H}^{+}$for de novo lipogenesis and protection from oxidative stress [71]. Previous studies in our laboratory have demonstrated that changes in the accumulation of G6PD mRNA in response to dietary status could not be attributed to changes in the rate of transcription or changes in mRNA stability $[72,73,145]$; demonstrating that G6PD mRNA is regulated post-transcriptionally. Further experiments demonstrated that these changes in G6PD mRNA abundance are the result of changes in splicing of the primary transcript $[69,72,75]$. Thus, G6PD represents an excellent model system to study mechanisms of nutrient-regulated splicing events.

Our laboratory has demonstrated that both SRSF3 phosphorylation and amount are increased in liver in response to insulin, and this effect is diminished following addition of the polyunsaturated fatty acid, arachidonic acid $[68,70]$. In addition, we have shown that arachidonic acid inhibits insulin-stimulated G6PD mRNA accumulation by stimulating the p38 MAPK pathway, which inhibits insulin signal transduction [68]. This is because activation of p38 MAPK stimulates Ser ${ }^{307}$ phosphorylation of IRS-1, which prevents activation of PI3K, thus blocking the activation of downstream effectors, including Akt. Since SRSF3 contains a consensus sequence for Akt phosphorylation in its RS domain and we showed that SRSF3 phosphorylation is increased upon insulin stimulation [70], we believed that SRSF3 could be a 
target of Akt or one of its effectors. A paper published in Molecular Cell in 2012 by the Fu group at the University of San Diego describes the Akt-SRPK-SR axis as a major regulator of alternative splicing in the nucleus through the epidermal growth factor (EGF) signal transduction pathway [146]. SRPKs are known to phosphorylate several SR proteins and regulate their activity $[111,112,114,132,136,146]$. When cells expressing an E1A splicing reporter were treated with EGF, major splicing changes were induced in a PI3K/Aktdependent manner. Intriguingly, EGF-induced splicing changes were not observed when SRPK1 was knocked down using RNA interference, but inhibition of mTOR had no effect on EGF-induced splicing. This suggests that regulation of SRPK1 by PI3K/Akt activity is a major driver of splicing changes and is thus likely to be the signal transduction pathway that regulates SRSF3 phosphorylation in response to insulin.

\section{HYPOTHESIS}

The objective of this dissertation was to determine whether the activity of SRSF3 changes in response to nutrients and also confirm that SRSF3 is the protein that mediates the increase in splicing of G6PD that occurs in response to refeeding and insulin treatment. The kind of alternative splicing observed in the G6PD gene, known as intron retention, is of particular importance, as we hypothesize that regulation of gene expression by intron retention is not unique to G6PD. Alternative splicing is regulated in a development and tissue-specific pattern, and in response to extracellular stimuli such as hormones and growth factors [43]. Thus, our hypothesis is that nutrients regulate splicing of hepatic genes by altering SR protein activity, and, that changes in the activity of SRSF3 in particular are significant for altering the expression of multiple genes in liver. 


\section{REFERENCES}

1. Crick, F., Central dogma of molecular biology. Nature, 1970. 227(5258): p. 561-3.

2. Moteki, S. and D. Price, Functional Coupling of Capping and Transcription of mRNA. Molecular Cell, 2002. 10(3): p. 599-609.

3. Beelman, C.A. and R. Parker, Degradation of mRNA in eukaryotes. Cell, 1995. 81(2): p. $179-83$.

4. Kim, H.J., et al., mRNA capping enzyme activity is coupled to an early transcription elongation. Mol Cell Biol, 2004. 24(14): p. 6184-93.

5. Proudfoot, N.J., A. Furger, and M.J. Dye, Integrating mRNA processing with transcription. Cell, 2002. 108(4): p. 501-12.

6. Kyburz, A., et al., Direct interactions between subunits of CPSF and the U2 snRNP contribute to the coupling of pre-mRNA 3' end processing and splicing. Mol Cell, 2006. 23(2): p. 195-205.

7. Wahle, E., A novel poly(A)-binding protein acts as a specificity factor in the second phase of messenger RNA polyadenylation. Cell, 1991. 66(4): p. 759-68.

8. Krause, S., et al., Immunodetection of poly $(A)$ binding protein II in the cell nucleus. Exp Cell Res, 1994. 214(1): p. 75-82.

9. Moore, M.J. and N.J. Proudfoot, Pre-mRNA processing reaches back to transcription and ahead to translation. Cell, 2009. 136(4): p. 688-700.

10. Proudfoot, N., New perspectives on connecting messenger RNA $3^{\prime}$ end formation to transcription. Curr Opin Cell Biol, 2004. 16(3): p. 272-8.

11. Proudfoot, N.J., How RNA polymerase II terminates transcription in higher eukaryotes. Trends Biochem Sci, 1989. 14(3): p. 105-10. 
12. Barabino, S.M. and W. Keller, Last but not least: regulated poly $(A)$ tail formation. Cell, 1999. 99(1): p. 9-11.

13. Atkins, J.F., R.F. Gesteland, and T. Cech, RNA worlds : from life's origins to diversity in gene regulation. 2011, Cold Spring Harbor, N.Y.: Cold Spring Harbor Laboratory Press. xv, $361 \mathrm{p}$.

14. Reed, R., Initial splice-site recognition and pairing during pre-mRNA splicing. Curr Opin Genet Dev, 1996. 6(2): p. 215-20.

15. Konarska, M.M. and P.A. Sharp, Interactions between small nuclear ribonucleoprotein particles in formation of spliceosomes. Cell, 1987. 49(6): p. 763-74.

16. Berget, S.M., Exon recognition in vertebrate splicing. J Biol Chem, 1995. 270(6): p. 2411-4.

17. Matera, A.G. and Z. Wang, A day in the life of the spliceosome. Nat Rev Mol Cell Biol, 2014. 15(2): p. 108-21.

18. Wahl, M.C., C.L. Will, and R. Luhrmann, The spliceosome: design principles of a dynamic RNP machine. Cell, 2009. 136(4): p. 701-18.

19. Cho, S., et al., Interaction between the RNA binding domains of Ser-Arg splicing factor 1 and U1-70K snRNP protein determines early spliceosome assembly. Proc Natl Acad Sci U S A, 2011. 108(20): p. 8233-8.

20. Sakharkar, M.K., V.T. Chow, and P. Kangueane, Distributions of exons and introns in the human genome. In Silico Biol, 2004. 4(4): p. 387-93.

21. Stamm, S., et al., A sequence compilation and comparison of exons that are alternatively spliced in neurons. Nucleic Acids Res, 1994. 22(9): p. 1515-26.

22. Black, D.L., Mechanisms of alternative pre-messenger RNA splicing. Annu Rev Biochem, 2003. 72: p. 291-336. 
23. De Conti, L., M. Baralle, and E. Buratti, Exon and intron definition in pre-mRNA splicing. Wiley Interdiscip Rev RNA, 2013. 4(1): p. 49-60.

24. Fourmann, J.B., et al., Dissection of the factor requirements for spliceosome disassembly and the elucidation of its dissociation products using a purified splicing system. Genes Dev, 2013. 27(4): p. 413-28.

25. Le Hir, H., et al., The spliceosome deposits multiple proteins 20-24 nucleotides upstream of mRNA exon-exon junctions. EMBO J, 2000. 19(24): p. 6860-9.

26. Moore, M.J., From birth to death: the complex lives of eukaryotic mRNAs. Science, 2005. 309(5740): p. 1514-8.

27. Stutz, F. and E. Izaurralde, The interplay of nuclear mRNP assembly, mRNA surveillance and export. Trends Cell Biol, 2003. 13(6): p. 319-27.

28. Dostie, J. and G. Dreyfuss, Translation is required to remove Y14 from mRNAs in the cytoplasm. Curr Biol, 2002. 12(13): p. 1060-7.

29. Le Hir, H., et al., The exon-exon junction complex provides a binding platform for factors involved in mRNA export and nonsense-mediated mRNA decay. EMBO J, 2001. 20(17): p. 4987-97.

30. Neu-Yilik, G. and A.E. Kulozik, NMD: multitasking between mRNA surveillance and modulation of gene expression. Adv Genet, 2008. 62: p. 185-243.

31. Hentze, M.W. and A.E. Kulozik, A perfect message: RNA surveillance and nonsensemediated decay. Cell, 1999. 96(3): p. 307-10.

32. Li, S. and M.F. Wilkinson, Nonsense surveillance in lymphocytes? Immunity, 1998. 8(2): p. 135-41. 
33. Nagy, E. and L.E. Maquat, A rule for termination-codon position within introncontaining genes: when nonsense affects RNA abundance. Trends Biochem Sci, 1998. 23(6): p. 198-9.

34. Behm-Ansmant, I. and E. Izaurralde, Quality control of gene expression: a stepwise assembly pathway for the surveillance complex that triggers nonsense-mediated mRNA decay. Genes Dev, 2006. 20(4): p. 391-8.

35. Goren, A., et al., Comparative analysis identifies exonic splicing regulatory sequences-The complex definition of enhancers and silencers. Mol Cell, 2006. 22(6): p. 769-81.

36. Sanford, J.R., J. Ellis, and J.F. Caceres, Multiple roles of arginine/serine-rich splicing factors in RNA processing. Biochem Soc Trans, 2005. 33(Pt 3): p. 443-6.

37. Erkelenz, S., et al., Position-dependent splicing activation and repression by $S R$ and hnRNP proteins rely on common mechanisms. RNA, 2013. 19(1): p. 96-102.

38. Prostko, C.R., R.S. Fritz, and R.F. Kletzien, Nutritional regulation of hepatic glucose-6phosphate dehydrogenase. Transient activation of transcription. Biochem J, 1989. 258(1): p. 295-9.

39. McCullough, A.J. and S.M. Berget, G triplets located throughout a class of small vertebrate introns enforce intron borders and regulate splice site selection. Mol Cell Biol, 1997. 17(8): p. 4562-71.

40. Chou, M.Y., et al., hnRNP H is a component of a splicing enhancer complex that activates a c-src alternative exon in neuronal cells. Mol Cell Biol, 1999. 19(1): p. 69-77.

41. Matlin, A.J., F. Clark, and C.W. Smith, Understanding alternative splicing: towards a cellular code. Nat Rev Mol Cell Biol, 2005. 6(5): p. 386-98.

42. Sanford, J.R. and J.F. Caceres, Pre-mRNA splicing: life at the centre of the central dogma. J Cell Sci, 2004. 117(Pt 26): p. 6261-3. 
43. Ravi, S., R.J. Schilder, and S.R. Kimball, Role of precursor mRNA splicing in nutrientinduced alterations in gene expression and metabolism. J Nutr, 2015. 145(5): p. 841-6.

44. Clark, F. and T.A. Thanaraj, Categorization and characterization of transcriptconfirmed constitutively and alternatively spliced introns and exons from human. Hum Mol Genet, 2002. 11(4): p. 451-64.

45. Wang, E.T., et al., Alternative isoform regulation in human tissue transcriptomes. Nature, 2008. 456(7221): p. 470-6.

46. Nilsen, T.W. and B.R. Graveley, Expansion of the eukaryotic proteome by alternative splicing. Nature, 2010. 463(7280): p. 457-463.

47. Boise, L.H., et al., $b c l-x$, a bcl-2-related gene that functions as a dominant regulator of apoptotic cell death. Cell, 1993. 74(4): p. 597-608.

48. Moore, M.J., et al., An alternative splicing network links cell-cycle control to apoptosis. Cell, 2010. 142(4): p. 625-36.

49. Cloutier, P., et al., Antagonistic effects of the SRp30c protein and cryptic 5' splice sites on the alternative splicing of the apoptotic regulator Bcl-x. J Biol Chem, 2008. 283(31): p. $21315-24$.

50. Revil, T., et al., Heterogeneous nuclear ribonucleoprotein $\mathrm{K}$ represses the production of pro-apoptotic Bcl-xS splice isoform. J Biol Chem, 2009. 284(32): p. 21458-67.

51. Zaghloul, N.A. and N. Katsanis, Mechanistic insights into Bardet-Biedl syndrome, a model ciliopathy. J Clin Invest, 2009. 119(3): p. 428-37.

52. Riazuddin, S.A., et al., A splice-site mutation in a retina-specific exon of BBS8 causes nonsyndromic retinitis pigmentosa. Am J Hum Genet, 2010. 86(5): p. 805-12.

53. Murphy, D., et al., Alternative Splicing Shapes the Phenotype of a Mutation in BBS8 To Cause Nonsyndromic Retinitis Pigmentosa. Mol Cell Biol, 2015. 35(10): p. 1860-70. 
54. Keren, H., G. Lev-Maor, and G. Ast, Alternative splicing and evolution: diversification, exon definition and function. Nat Rev Genet, 2010. 11(5): p. 345-55.

55. Lund, M. and J. Kjems, Defining a 5' splice site by functional selection in the presence and absence of U1 snRNA 5' end. RNA, 2002. 8(2): p. 166-79.

56. Lev-Maor, G., et al., The birth of an alternatively spliced exon: 3' splice-site selection in Alu exons. Science, 2003. 300(5623): p. 1288-91.

57. Massiello, A., et al., Identification of two RNA cis-elements that function to regulate the 5' splice site selection of Bcl-x pre-mRNA in response to ceramide. J Biol Chem, 2004. 279(16): p. 15799-804.

58. Coelho, M.B. and C.W.J. Smith, Alternative Splice Site Selection, in Alternative premRNA Splicing. 2012, Wiley-VCH Verlag GmbH \& Co. KGaA. p. 93-107.

59. Goo, Y.H. and T.A. Cooper, CUGBP2 directly interacts with U2 17S snRNP components and promotes U2 snRNA binding to cardiac troponin T pre-mRNA. Nucleic Acids Res, 2009. 37(13): p. 4275-86.

60. Cooper, T.A. and C.P. Ordahl, $A$ single cardiac troponin $T$ gene generates embryonic and adult isoforms via developmentally regulated alternate splicing. J Biol Chem, 1985. 260(20): p. $11140-8$.

61. McAuliffe, J.J., L.Z. Gao, and R.J. Solaro, Changes in myofibrillar activation and troponin C Ca2+ binding associated with troponin $T$ isoform switching in developing rabbit heart. Circ Res, 1990. 66(5): p. 1204-16.

62. Braunschweig, U., et al., Widespread intron retention in mammals functionally tunes transcriptomes. Genome Res, 2014. 24(11): p. 1774-86.

63. Muhlemann, O., et al., Precursor RNAs harboring nonsense codons accumulate near the site of transcription. Mol Cell, 2001. 8(1): p. 33-43. 
64. Wang, J., et al., A quality control pathway that down-regulates aberrant T-cell receptor (TCR) transcripts by a mechanism requiring UPF2 and translation. J Biol Chem, 2002. 277(21): p. 18489-93.

65. Wong, J.J., et al., Orchestrated intron retention regulates normal granulocyte differentiation. Cell, 2013. 154(3): p. 583-95.

66. Bicknell, A.A., et al., Introns in UTRs: why we should stop ignoring them. Bioessays, 2012. 34(12): p. 1025-34.

67. Szeszel-Fedorowicz, W., et al., An exonic splicing silencer is involved in the regulated splicing of glucose 6-phosphate dehydrogenase mRNA. J Biol Chem, 2006. 281(45): p. 34146-58.

68. Talukdar, I., W. Szeszel-Fedorowicz, and L.M. Salati, Arachidonic acid inhibits the insulin induction of glucose-6-phosphate dehydrogenase via p38 MAP kinase. J Biol Chem, 2005. 280(49): p. 40660-7.

69. Tao, H., et al., Inhibition of the splicing of glucose-6-phosphate dehydrogenase precursor mRNA by polyunsaturated fatty acids. J Biol Chem, 2002. 277(34): p. $31270-8$.

70. Walsh, C.M., et al., Serine arginine splicing factor 3 is involved in enhanced splicing of glucose-6-phosphate dehydrogenase RNA in response to nutrients and hormones in liver. J Biol Chem, 2013. 288(4): p. 2816-28.

71. Salati, L.M. and B. Amir-Ahmady, Dietary regulation of expression of glucose-6phosphate dehydrogenase. Annu Rev Nutr, 2001. 21: p. 121-40.

72. Amir-Ahmady, B. and L.M. Salati, Regulation of the Processing of Glucose-6phosphate Dehydrogenase mRNA by Nutritional Status. Journal of Biological Chemistry, 2001. 276(13): p. 10514-10523. 
73. Stabile, L.P., et al., Posttranscriptional regulation of glucose-6-phosphate dehydrogenase by dietary polyunsaturated fat. Arch Biochem Biophys, 1996. 332(2): p. 269-79.

74. Cyphert, T.J., et al., Starvation actively inhibits splicing of glucose-6-phosphate dehydrogenase mRNA via a bifunctional ESE/ESS element bound by hnRNP K. Biochim Biophys Acta, 2013. 1829(9): p. 905-15.

75. Griffith, B.N., et al., Identification of $h n R N P s K, L$ and $A 2 / B 1$ as candidate proteins involved in the nutritional regulation of mRNA splicing. Biochim Biophys Acta, 2006. 1759(11-12): p. 552-61.

76. Manley, J.L. and R. Tacke, SR proteins and splicing control. Genes Dev, 1996. 10(13): p. 1569-79.

77. Martinez-Contreras, R., et al., hnRNP proteins and splicing control. Adv Exp Med Biol, 2007. 623: p. 123-47.

78. Das, R., et al., SR proteins function in coupling RNAP II transcription to pre-mRNA splicing. Mol Cell, 2007. 26(6): p. 867-81.

79. Fu, X.D., Specific commitment of different pre-mRNAs to splicing by single SR proteins. Nature, 1993. 365(6441): p. 82-5.

80. Sanford, J.R., et al., A novel role for shuttling SR proteins in mRNA translation. Genes Dev, 2004. 18(7): p. 755-68.

81. Sanford, J.R., et al., Splicing factor SFRS1 recognizes a functionally diverse landscape of RNA transcripts. Genome Res, 2009. 19(3): p. 381-94.

82. Sen, S., H. Jumaa, and N.J. Webster, Splicing factor SRSF3 is crucial for hepatocyte differentiation and metabolic function. Nat Commun, 2013. 4: p. 1336. 
83. Sen, S., I. Talukdar, and N.J. Webster, SRp20 and CUG-BP1 modulate insulin receptor exon 11 alternative splicing. Mol Cell Biol, 2009. 29(3): p. 871-80.

84. Tacke, R. and J.L. Manley, The human splicing factors ASF/SF2 and SC35 possess distinct, functionally significant RNA binding specificities. EMBO J, 1995. 14(14): p. 3540-51.

85. Tacke, R. and J.L. Manley, Determinants of SR protein specificity. Curr Opin Cell Biol, 1999. 11(3): p. 358-62.

86. Twyffels, L., C. Gueydan, and V. Kruys, Shuttling SR proteins: more than splicing factors. FEBS J, 2011. 278(18): p. 3246-55.

87. Wang, J. and J.L. Manley, Overexpression of the SR proteins ASF/SF2 and SC35 influences alternative splicing in vivo in diverse ways. RNA, 1995. 1(3): p. 335-46.

88. Busch, A. and K.J. Hertel, Evolution of SR protein and hnRNP splicing regulatory factors. Wiley Interdisciplinary Reviews: RNA, 2011.

89. Caceres, J.F., et al., Role of the modular domains of SR proteins in subnuclear localization and alternative splicing specificity. J Cell Biol, 1997. 138(2): p. 225-38.

90. Graveley, B.R. and K.J. Hertel, SR Proteins, in eLS. 2001, John Wiley \& Sons, Ltd.

91. Misteli, T., et al., Serine phosphorylation of SR proteins is required for their recruitment to sites of transcription in vivo. J Cell Biol, 1998. 143(2): p. 297-307.

92. Dreyfuss, G., et al., hnRNP proteins and the biogenesis of mRNA. Annu Rev Biochem, 1993. 62: p. 289-321.

93. Long, J.C. and J.F. Caceres, The SR protein family of splicing factors: master regulators of gene expression. Biochem J, 2009. 417(1): p. 15-27.

94. Zhong, X.Y., et al., SR proteins in vertical integration of gene expression from transcription to RNA processing to translation. Mol Cell, 2009. 35(1): p. 1-10. 
95. Lin, S. and X.D. Fu, SR proteins and related factors in alternative splicing. Adv Exp Med Biol, 2007. 623: p. 107-22.

96. Juge, F., et al., The SR protein B52/SRp55 is required for DNA topoisomerase I recruitment to chromatin, mRNA release and transcription shutdown. PLoS Genet, 2010. 6(9): p. e1001124.

97. Pinol-Roma, S. and G. Dreyfuss, hnRNP proteins: localization and transport between the nucleus and the cytoplasm. Trends Cell Biol, 1993. 3(5): p. 151-5.

98. Chaudhury, A., P. Chander, and P.H. Howe, Heterogeneous nuclear ribonucleoproteins (hnRNPs) in cellular processes: Focus on hnRNP E1's multifunctional regulatory roles. RNA, 2010. 16(8): p. 1449-62.

99. Zhu, J., A. Mayeda, and A.R. Krainer, Exon identity established through differential antagonism between exonic splicing silencer-bound hnRNP A1 and enhancer-bound SR proteins. Mol Cell, 2001. 8(6): p. 1351-61.

100. Han, S.P., Y.H. Tang, and R. Smith, Functional diversity of the hnRNPs: past, present and perspectives. Biochem J, 2010. 430(3): p. 379-92.

101. Siomi, H., et al., The pre-mRNA binding K protein contains a novel evolutionarily conserved motif. Nucleic Acids Res, 1993. 21(5): p. 1193-8.

102. Nakielny, S. and G. Dreyfuss, The hnRNP C proteins contain a nuclear retention sequence that can override nuclear export signals. J Cell Biol, 1996. 134(6): p. 136573.

103. Michael, W.M., P.S. Eder, and G. Dreyfuss, The K nuclear shuttling domain: a novel signal for nuclear import and nuclear export in the hnRNP K protein. EMBO J, 1997. 16(12): p. 3587-98. 
104. Manley, J.L. and A.R. Krainer, A rational nomenclature for serine/arginine-rich protein splicing factors (SR proteins). Genes Dev, 2010. 24(11): p. 1073-4.

105. Lamond, A.I. and D.L. Spector, Nuclear speckles: a model for nuclear organelles. Nat Rev Mol Cell Biol, 2003. 4(8): p. 605-12.

106. Edmond, V., et al., Acetylation and phosphorylation of SRSF2 control cell fate decision in response to cisplatin. EMBO J, 2011. 30(3): p. 510-23.

107. Sinha, R., et al., Arginine methylation controls the subcellular localization and functions of the oncoprotein splicing factor SF2/ASF. Mol Cell Biol, 2010. 30(11): p. 2762-74.

108. Kim, S., et al., Specific SR protein-dependent splicing substrates identified through genomic SELEX. Nucleic Acids Res, 2003. 31(7): p. 1955-61.

109. Bradley, T., M.E. Cook, and M. Blanchette, SR proteins control a complex network of RNA-processing events. RNA, 2015. 21(1): p. 75-92.

110. Ghosh, G. and J.A. Adams, Phosphorylation mechanism and structure of serinearginine protein kinases. FEBS Journal, 2011. 278(4): p. 587-597.

111. Aubol, B.E. and J.A. Adams, Applying the brakes to multisite SR protein phosphorylation: substrate-induced effects on the splicing kinase SRPK1. Biochemistry, 2011. 50(32): p. 6888-900.

112. Colwill, K., et al., SRPK1 and Clk/Sty protein kinases show distinct substrate specificities for serine/arginine-rich splicing factors. J Biol Chem, 1996. 271(40): p. 24569-75.

113. Jiang, K., et al., Akt2 Regulation of Cdc2-Like Kinases (Clk/Sty), Serine/Arginine-Rich (SR) Protein Phosphorylation, and Insulin-Induced Alternative Splicing of PKCCEsII Messenger Ribonucleic Acid. Endocrinology, 2009. 150(5): p. 2087-2097. 
114. Koizumi, J., et al., The Subcellular Localization of SF2/ASF Is Regulated by Direct Interaction with SR Protein Kinases (SRPKs). Journal of Biological Chemistry, 1999. 274(16): p. 11125-11131.

115. Lai, M.C., et al., A human importin-beta family protein, transportin-SR2, interacts with the phosphorylated RS domain of SR proteins. J Biol Chem, 2000. 275(11): p. 7950-7.

116. Sanford, J.R., et al., Reversible phosphorylation differentially affects nuclear and cytoplasmic functions of splicing factor 2/alternative splicing factor. Proc Natl Acad Sci U S A, 2005. 102(42): p. 15042-7.

117. Tacke, R., Y. Chen, and J.L. Manley, Sequence-specific RNA binding by an SR protein requires RS domain phosphorylation: creation of an SRp40-specific splicing enhancer. Proc Natl Acad Sci U S A, 1997. 94(4): p. 1148-53.

118. Zhong, X.-Y., et al., Regulation of SR protein phosphorylation and alternative splicing by modulating kinetic interactions of SRPK1 with molecular chaperones. Genes \& Development, 2009. 23(4): p. 482-495.

119. Misteli, T., RNA splicing: What has phosphorylation got to do with it? Curr Biol, 1999. 9(6): p. R198-200.

120. Howard, J.M. and J.R. Sanford, The RNAissance family: SR proteins as multifaceted regulators of gene expression. Wiley Interdiscip Rev RNA, 2015. 6(1): p. 93-110.

121. Jakubauskiene, E., et al., Increased Serine-Arginine (SR) Protein Phosphorylation Changes Pre-mRNA Splicing in Hypoxia. J Biol Chem, 2015. 290(29): p. 18079-89.

122. Misteli, T. and D.L. Spector, Serine/threonine phosphatase 1 modulates the subnuclear distribution of pre-mRNA splicing factors. Molecular Biology of the Cell, 1996. 7(10): p. 1559-72. 
123. Colwill, K., et al., The Clk/Sty protein kinase phosphorylates SR splicing factors and regulates their intranuclear distribution. EMBO J, 1996. 15(2): p. 265-75.

124. Blaustein, M., et al., Concerted regulation of nuclear and cytoplasmic activities of SR proteins by AKT. Nat Struct Mol Biol, 2005. 12(12): p. 1037-44.

125. Mermoud, J.E., P.T. Cohen, and A.I. Lamond, Regulation of mammalian spliceosome assembly by a protein phosphorylation mechanism. EMBO J, 1994. 13(23): p. 567988.

126. Graveley, B.R., Sorting out the complexity of SR protein functions. RNA, 2000. 6(9): $p$. $1197-211$.

127. Hertel, K.J. and T. Maniatis, Serine-arginine (SR)-rich splicing factors have an exonindependent function in pre-mRNA splicing. Proc Natl Acad Sci U S A, 1999. 96(6): p. 2651-5.

128. Aubol, B.E. and J.A. Adams, Recruiting a silent partner for activation of the protein kinase SRPK1. Biochemistry, 2014. 53(28): p. 4625-34.

129. Caceres, J.F., G.R. Screaton, and A.R. Krainer, A specific subset of SR proteins shuttles continuously between the nucleus and the cytoplasm. Genes \& Development, 1998. 12(1): p. 55-66.

130. Twyffels, L., C. Gueydan, and V. Kruys, Shuttling SR proteins: more than splicing factors. FEBS Journal, 2011. 278(18): p. 3246-3255.

131. Huang, Y., et al., SR splicing factors serve as adapter proteins for TAP-dependent mRNA export. Mol Cell, 2003. 11(3): p. 837-43.

132. Wang, H.Y., et al., SRPK2: a differentially expressed SR protein-specific kinase involved in mediating the interaction and localization of pre-mRNA splicing factors in mammalian cells. J Cell Biol, 1998. 140(4): p. 737-50. 
133. Jumaa, H., G. Wei, and P.J. Nielsen, Blastocyst formation is blocked in mouse embryos lacking the splicing factor SRp20. Curr Biol, 1999. 9(16): p. 899-902.

134. Fitzgerald, K.D. and B.L. Semler, Re-localization of cellular protein SRp20 during poliovirus infection: bridging a viral IRES to the host cell translation apparatus. PLoS Pathog, 2011. 7(7): p. e1002127.

135. Verma, D., et al., Epstein-Barr Virus SM protein utilizes cellular splicing factor SRp20 to mediate alternative splicing. J Virol, 2010. 84(22): p. 11781-9.

136. Sciabica, K.S., Q.J. Dai, and R.M. Sandri-Goldin, ICP27 interacts with SRPK1 to mediate HSV splicing inhibition by altering SR protein phosphorylation. EMBO J, 2003. 22(7): p. 1608-19.

137. Escudero-Paunetto, L., et al., SR proteins SRp20 and $9 G 8$ contribute to efficient export of herpes simplex virus 1 mRNAs. Virology, 2010. 401(2): p. 155-64.

138. Wong, R.W., et al., Digoxin suppresses HIV-1 replication by altering viral RNA processing. PLoS Pathog, 2013. 9(3): p. e1003241.

139. Jia, R., et al., Control of the papillomavirus early-to-late switch by differentially expressed SRp20. J Virol, 2009. 83(1): p. 167-80.

140. Liang, H., R.S. Tuan, and P.A. Norton, Overexpression of SR proteins and splice variants modulates chondrogenesis. Exp Cell Res, 2007. 313(8): p. 1509-17.

141. Galiana-Arnoux, D., et al., The CD44 alternative v9 exon contains a splicing enhancer responsive to the SR proteins 9G8, ASF/SF2, and SRp20. J Biol Chem, 2003. 278(35): p. 32943-53.

142. Lim, L.P. and P.A. Sharp, Alternative splicing of the fibronectin EllIB exon depends on specific TGCATG repeats. Mol Cell Biol, 1998. 18(7): p. 3900-6. 
143. Jumaa, H. and P.J. Nielsen, The splicing factor SRp20 modifies splicing of its own $m R N A$ and ASF/SF2 antagonizes this regulation. EMBO J, 1997. 16(16): p. 5077-85.

144. Jumaa, H. and P.J. Nielsen, Regulation of SRp20 exon 4 splicing. Biochim Biophys Acta, 2000. 1494(1-2): p. 137-43.

145. Amir-Ahmady, B. and L.M. Salati, Regulation of the processing of glucose-6phosphate dehydrogenase mRNA by nutritional status. J Biol Chem, 2001. 276(13): p. 10514-23.

146. Zhou, Z., et al., The Akt-SRPK-SR Axis Constitutes a Major Pathway in Transducing EGF Signaling to Regulate Alternative Splicing in the Nucleus. Molecular Cell, 2012. 47(3): p. $422-433$. 


\section{PREFACE}

In 2013, we published the following article in the Journal of Biological Chemistry in which we definitively established SRSF3 as a refeeding-enhanced splicing factor. The project was started by a previous graduate student, Dr. Callee Walsh, and completed by me. My specific contributions range from troubleshooting and running new blots and RT-PCR in figure $1 \mathrm{~d}$ and $1 \mathrm{e}$, to performing the experiments, data collection and analyses in figures 4,5 , and 7 .

The basis of the paper is that changes in the accumulation of glucose-6-phosphate dehydrogenase (G6PD) mRNA are caused by changes in splicing in response to nutrient availability-specifically starvation and refeeding. A splicing regulatory element within exon 12 of the G6PD transcript is required for splicing-related changes in G6PD expression in response to nutrients. When I joined the projected, Dr. Walsh had performed the ChIP experiments that indicated that several serine/arginine-rich (SR) proteins may be binding to the G6PD regulatory element in response to changes in nutrient status.

To determine which specific SR proteins affected the splicing of G6PD mRNA, I used an siRNA knockdown approach. For these experiments, I used HepG2 cells that stably expresses a G6PD splicing reporter construct (Figure 5a). I was able to show that an $80 \%$ decrease in SRSF3 protein (Figure 5b-c), resulted in a $60 \%$ decrease in splicing reporter mRNA (Figure 5d). Conversely, a $70 \%$ depletion of the related protein, SRSF4, did not significantly affect the amount of G6PD mRNA (Figure 5b-d). These experiments helped drive us to focus future experiments on SRSF3 as the primary regulator of G6PD splicing.

To confirm that SRSF3 bound directly to the G6PD regulatory element, I adapted and optimized a technique called RNA immunoprecipitation (RIP) for our laboratory. Using the RIP 
technique in HepG2 cells, I detected binding of both endogenous SRSF3 and a FLAG-tagged SRSF3 to the exon 12 regulatory element (Figs. 3A and B). In addition to this cell line model, I performed SRSF3 RIP on whole liver extracts prepared from starved and refed mice. I was able to demonstrate that SRSF3 binds to the regulatory element in whole liver, and that binding is increased during refeeding and decreased during starvation (Fig. 3C). The increased binding of SRSF3 corresponds to a 7-fold increase in G6PD mRNA abundance (Fig. 3D). Furthermore, in an RNA EMSA using purified SRSF3 recombinant protein, I demonstrated that SRSF3 binds to the G6PD regulatory element in a sequence-specific manner (Figure 4).

These were a novel findings in that binding of SRSF3 (or any other SR protein) had not been demonstrated to be regulated by physiological signals. Thus, we concluded that binding of SRSF3 to the regulatory element mediates the nutrient regulation of G6PD mRNA splicing, at least in part. Importantly, we identified a new intracellular mechanism for nutrient regulation of gene expression. 
Serine Arginine Splicing Factor 3 (SRSF3) is involved in enhanced splicing of glucose-6phosphate dehydrogenase (G6PD) RNA in response to nutrients and hormones in liver*

\section{Callee M. Walsh ${ }^{1}$, Amanda L. Suchanek ${ }^{1}$, Travis J. Cyphert, Alison B. Kohan, Wioletta Szeszel-Fedorowicz, and Lisa M. Salati ${ }^{2 S}$}

Department of Biochemistry, West Virginia University, Morgantown, WV

To whom correspondence should be addressed: Lisa M. Salati; Department of Biochemistry, One Medical Center Drive, PO Box 9142 Health Sciences Center, Morgantown, WV 26506; Phone: (304) 293-7759; Fax: (304) 293-6846; E-mail: Lsalati@ hsc.wvu.edu

Keywords: splicing, SR protein, SRSF3, insulin, fatty acid, glucose-6-phosphate dehydrogenase, alternative splicing, intron retention, exonic splicing enhancer, hepatocytes, chromatin immunoprecipitation, RNA immunoprecipitation, RNA EMSA

Background: Regulation of G6PD expression by nutrients occurs by changes in accumulation of spliced mRNA without changes in transcriptional activity of the gene.

Results: Refeeding enhances SRSF3 binding to G6PD mRNA. Loss of SRSF3 inhibits G6PD expression.

Conclusion: SRSF3 is a target for nutritional regulation of splicing.

Significance: Regulation of RNA splicing is a novel target for nutrient action. 


\section{FOOTNOTES}

* This work was supported by National Institutes of Health Grant DK46897 to LMS and T32 HL090610 to ABK, and a grant from the West Virginia Graduate Student Fellowships in Science, Technology, Engineering and Math (STEM) program to CMW.

${ }^{\mathrm{S}}$ This article contains supplemental Table 1 and Figs. 1-3.

${ }^{1}$ These 2 authors have contributed equally to the conduct of these experiments.

${ }^{2}$ To whom correspondence should be addressed: Department of Biochemistry, One Medical Center Drive, PO Box 9142 Health Sciences Center, Morgantown, WV 26506; Phone: (304) 293-7759; Fax: (304) 293-6846; E-mail: Lsalati@hsc.wvu.edu

${ }^{3}$ Abbreviations: SRSF, serine arginine splicing factor; G6PD, glucose-6-phosphate dehydrogenase; SR, serine-arginine rich; ESE, exonic splicing enhancer; RS domain, arginineserine rich domain; HEX ${ }^{\mathrm{TM}}$, 5'-hexachlorofluorescein; glutathione S-transferase, GST; RIP, RNA immunoprecipitation; PEPCK, phosphoenolpyruvate carboxykinase; Pol II, polymerase II; hnRNP, heterogeneous nuclear ribonucleoprotein; pre-mRNA, precursor-mRNA 


\section{ABSTRACT}

Expression of G6PD is controlled by changes in the degree of splicing of the G6PD mRNA in response to nutrients in the diet. This regulation involves an exonic splicing enhancer $(E S E)^{3}$ in exon 12 of the mRNA. Using the G6PD model, we demonstrate that nutrients and hormones control the activity of SR proteins, a family of splicing co-activators, and thereby regulate the splicing of G6PD mRNA. In primary rat hepatocyte cultures, insulin increased the amount of phosphorylated SR proteins and this effect was counteracted by arachidonic acid. The results of RNA affinity analysis with nuclear extracts from intact liver demonstrated that the SR proteins SRSF3 and SRSF4 bound to the G6PD ESE. Consequently, siRNA-mediated depletion of SRSF3, but not SRSF4, in liver cells inhibited accumulation of both mRNA expressed from a minigene containing exon 12 and the endogenous G6PD mRNA. Consistent with the functional role of SRSF3 in regulating splicing, SRSF3 was observed to bind to the ESE in both intact cells and in animals using RIP analysis. Furthermore, refeeding significantly increased the binding of SRSF3 coincident with increased splicing and expression of G6PD. Together, these data establish that nutritional regulation of SRSF3 activity is involved in the differential splicing of the G6PD transcript in response to nutrients. Nutritional regulation of other SR proteins presents a regulatory mechanism that could cause widespread changes in mRNA splicing. Nutrients are therefore novel regulators of mRNA splicing. 


\section{INTRODUCTION}

Nutritional status is a central regulator of cellular function. At a cellular level, the macronutrients, carbohydrate, amino acids and fatty acids can modulate signal transduction pathways and gene expression. Nutrients can also affect circulating levels of hormones in animals. While key enzymes in the pathways of intermediary metabolism are primary targets for regulation by nutritional status, our current understanding of the molecular details causing this regulation have emphasized transcriptional mechanisms or changes in the kinetic properties of key regulatory enzymes. We have gathered a body of data indicating that alternative RNA splicing is a significant, yet underappreciated mechanism by which nutrients regulate gene expression $(1,2)$.

Alternative splicing is regulated in a developmental and tissue-specific pattern, and in response to extracellular stimuli such as hormones and growth factors $(3,4)$. Cis-acting elements in the RNA and trans-acting factors are key determinants of this process; mutations in these elements or changes in the amount or activity of trans-acting proteins alter the cellular pattern of mRNA and the protein isoforms that they encode. Greater than $90 \%$ of human transcripts appear to undergo alternative splicing resulting in changes in the amount of a protein expressed, production of multiple proteins from a single gene, and the presence or absence of specific domains within a protein (5). Thus the consequences of regulating this process have large implications for cellular fate and metabolism.

Serine-arginine rich (SR) proteins are a family of trans-acting splicing factors that activate splicing and play key roles in the regulation of alternative splicing (6). The canonical SR protein family members (SRSFs 1 to 12 ) are RNA-binding proteins with long, unstructured repeats of alternating serines and arginines forming RS domains in their C-terminus. The members of this family also contain one or two RNA recognition motifs in the N-terminus (6). 
SR proteins play a critical role in the recognition of alternatively included exons as well as constitutive exons that have weak splice sites (6). These proteins enhance the splicing process by binding to exonic splicing enhancers (ESEs) to recruit components of the spliceosome, specifically $\mathrm{U} 1 \mathrm{snRNP}, \mathrm{U} 2 \mathrm{AF}^{35}$, and $\mathrm{U} 2 \mathrm{snRNP}$ to intronic splice sites and/or by inhibiting the binding of negative regulators of splicing $(6,7)$. The serines in the RS domains can be phosphorylated (6). Phosphorylation enhances the ability of the SR protein to recruit components of the spliceosome to splice sites, regulates the intra-cellular and intra-nuclear localization of SR proteins, and enhances their binding of the proteins to RNA (8). SR proteins are known to be substrates of several kinases including SRPK, Clk/Sty and Akt, and as such, are candidates for mediating the impact of extracellular signals upon the splicing process (911). The involvement of hormones and other extracellular signals in regulating splicing factor activity is poorly understood.

G6PD is an enzyme that is integral to the process of de novo lipogenesis in liver and adipose tissue by its generation of reducing equivalents in the form of $\mathrm{NADPH}+\mathrm{H}^{+}(12)$. We previously showed that nutritional status of the animal regulates expression of G6PD by changes in the efficiency by which the primary transcript is spliced and without changes in the transcriptional rate of the gene (13-15). Starvation or consumption of a diet containing polyunsaturated fatty acids reduces the efficiency of intron removal from the primary G6PD transcript (15), subsequently decreasing expression of the enzyme and therefore, fewer reducing equivalents are available for lipogenesis. G6PD precursor-mRNA (pre-mRNA) containing retained introns adjacent to exon 12 accumulates in the nucleus when splicing is inhibited (15). Feeding a high-carbohydrate, low-fat diet to rodents after a short-term fast induces efficient splicing of the mRNA and mature mRNA accumulates in the absence of a change in the accumulation of the primary transcript. The increase in G6PD mRNA results in 
greater expression of the enzyme, and an increase in lipogenic capacity as compared to fasted animals (13). These in vivo feeding experiments are recapitulated in primary rat hepatocytes in culture in which treatment with insulin induces the accumulation of spliced G6PD mRNA, and the polyunsaturated fatty acid, arachidonic acid, attenuates mRNA splicing $(2,15,16)$. A splicing regulatory element in exon 12 of the G6PD transcript mediates the effect of nutrients upon the splicing of this pre-mRNA (2). The absence of transcriptional regulation of this gene by nutrients has made it a useful model for deciphering the molecular mechanisms involved in this posttranscriptional regulation.

We hypothesize that these splicing-related changes involve the differential binding of splicing regulatory proteins to the regulatory element in exon 12 . In this report, we demonstrate that SR proteins are candidates for nutrient regulation of splicing. In this regard, SR protein amount and phosphorylation in rat hepatocytes is increased by insulin, and arachidonic acid inhibits this effect. Within the family of SR proteins, SRSF3 binds to the splicing regulatory element and the binding of SRSF3 to the regulatory element in intact liver decreases during starvation and increases upon refeeding. Loss of SRSF3 decreases accumulation of spliced G6PD mRNA. These data are the first to demonstrate that nutritional status can regulate SR protein activity and introduces a new paradigm by which mRNA splicing can be regulated. 


\section{EXPERIMENTAL PROCEDURES}

All animal experiments were conducted in conformity with the Public Health Service policy on Humane Care and Use of Laboratory Animals, additionally; the Institutional Animal Care and Use Committee of the Division of Laboratory Animal Resources at West Virginia University approved all experimental procedures.

Hepatocyte preparation - Hepatocytes were isolated from male Sprague-Dawley rats (approximately $200 \mathrm{~g}$ ) as previously described (15). Hepatocytes $\left(3 \times 10^{6}\right)$ were plated in collagen-coated $60 \mathrm{~mm}$ dishes containing Hi/Wo/BA medium (Waymouth MB752/l plus $20 \mathrm{mM}$ Hepes, $\mathrm{pH} 7.4,0.5 \mathrm{mM}$ serine, $0.5 \mathrm{mM}$ alanine, $0.2 \% \mathrm{BSA}$ ) plus $5 \%$ newborn calf serum. After $4 \mathrm{~h}$, the cells were washed with serum-free medium and a Matrigel ${ }^{\mathrm{TM}}$ overlay was added $(0.3$ $\mathrm{mg} / \mathrm{ml}$; BD Pharmingen). After $24 \mathrm{~h}$ in culture, the medium was replaced with $\mathrm{Hi} / \mathrm{Wo} / \mathrm{Ba}$ alone or $\mathrm{Hi} / \mathrm{Wo} / \mathrm{Ba}$ containing $80 \mathrm{nM}$ insulin or $80 \mathrm{nM}$ insulin plus $175 \mu \mathrm{M}$ arachidonic acid conjugated to BSA (15) and the medium was replenished every $12 \mathrm{~h}$. The cells were harvested for RNA isolation or extract preparation after $24 \mathrm{~h}$ with these treatments. Nuclear extracts (from 6-12 plates per treatment) were prepared by the method of Dignam et al. (17). The proteins were separated by size using SDS-PAGE and subjected to western analysis as described below.

Nuclear extract preparation from whole liver - Male Sprague-Dawley rats (approximately $200 \mathrm{~g}$ ) were either fasted for $24 \mathrm{~h}$ or fasted for $24 \mathrm{~h}$ and then refed a high-glucose diet (fatfree/high-glucose USB diet, \#1810092, Purina Mills containing 1\% safflower oil as a source of essential fatty acid) for $16 \mathrm{~h}$ prior to euthanasia. Liver nuclei were isolated by the method of Schibler et al. (18) and the nuclear proteins were extracted using the Dignam protocol (17). All buffers contained protease and phosphatase inhibitors ( $1 \mathrm{mM} \mathrm{Na}_{3} \mathrm{VO}_{4}, 1 \mathrm{mM}$ PMSF, $50 \mathrm{mM}$ $\mathrm{NaF}, 1 \mathrm{mM}$ benzamidine, $0.5 \mu \mathrm{g} / \mathrm{ml}$ leupeptin, $2 \mu \mathrm{g} / \mathrm{ml}$ aprotinin, $10 \mathrm{mM} \beta$-glycerophosphate, 
and $0.1 \mathrm{mM} \mathrm{Na}_{2} \mathrm{MoO}_{4}$ ). A portion of the liver was used for measurement of G6PD mRNA abundance.

RNA affinity assay - RNA oligonucleotides corresponding to nucleotides 43-72 and 7993 of exon 12 of G6PD were purchased from IDT. The RNA oligonucleotides were attached to adipic acid dihydrazide-agarose beads (Sigma) as previously described (19). Each RNA-bead complex was mixed with binding buffer containing rat liver nuclear extract ( $250 \mu \mathrm{g}$ protein), 20 mM HEPES, pH 7.4, 9\% glycerol, 70 mM KCl, 0.2 mM EDTA pH 8.0, $1 \mu \mathrm{g} / \mathrm{ml}$ tRNA, $2.5 \mathrm{mM}$ ATP, $2 \mathrm{mM} \mathrm{MgCl}$, and $0.2 \mathrm{mM} \mathrm{DTT}$. The binding reactions were incubated at $30^{\circ} \mathrm{C}$ for $30 \mathrm{~min}$, after which the bead mixtures were placed into columns. Unbound proteins were washed from the RNA-bead complexes with 20 mM HEPES, pH 7.4, 5\% glycerol, $0.2 \mathrm{mM}$ EDTA pH 8.0, and $0.1 \mathrm{mM}$ DTT. The bound proteins were eluted by gravity flow in wash buffer plus $250 \mathrm{mM} \mathrm{KCl}$. The appropriate concentration of KCL for elution was determined empirically. The eluate was desalted and concentrated with Amicon ${ }^{\circledR}$ Ultra centrifugal filter devices $(3,000 \mathrm{MWCO}$, Millipore). The concentrated proteins were separated by size using SDS-PAGE and bound SR proteins were detected by western analysis.

Western analysis - Following PAGE, the proteins were transferred to PVDF membranes (Bio-Rad) and probed with the antibodies indicated in the figure legends. Phosphorylated SR proteins were detected using the supernatant from hybridoma cells (ATCC) expressing the monoclonal antibody mAb104 or a commercially prepared monoclonal antibody $1 \mathrm{H} 4$ (Invitrogen). These 2 antibodies detect SRSFs 1-6. Total SR protein abundance was measured using the monoclonal antibody $16 \mathrm{H} 3$ (Invitrogen; referred to as Pan-SR) that detects SRSF4, SRSF6, SRSF5 and SRSF3, regardless of phosphorylation state. The amount of SRSF3 was also measured using a specific anti-SRSF3 antibody (7B4, Invitrogen). Lamin A/C (Cell Signaling) was used as a loading control. Secondary antibodies were conjugated to 
horseradish peroxidase and the signals were detected using ECL Plus (GE Healthcare), followed by visualization on film and a Typhoon 9410 Imager (GE Healthcare). Signals were quantified with ImageJ (NIH) or ImageQuant TL (Molecular Dynamics), respectively.

RNA isolation and measurement - Total RNA was isolated using TRI Reagent ${ }^{\circledR}$ (Molecular Research Center) and digested with DNase I (Turbo DNA-free, Invitrogen) according to the manufacturers protocol. G6PD and cyclophilin B mRNAs were quantified by real-time RT-PCR (iCYCLER, Bio-Rad) using the QuantiTect SYBR Green kit (Qiagen) and the primers and probes listed in supplemental Table 1. The amount of each mRNA was calculated using a relative standard curve.

Chromatin Immunoprecipitation (ChIP) - Male C57BL/6 mice (6 weeks of age) were fed the high-carbohydrate diet as described above for one week. Mice were starved for $18 \mathrm{~h}$ (starved group) or were starved for $18 \mathrm{~h}$ and refed the high-carbohydrate diet for $12 \mathrm{~h}$ (refed group) prior to euthanasia. The crosslinking reaction and ChIP assay are modifications of existing protocols $(20,21)$. Immediately after euthanasia, the livers were removed and immersed in 4 volumes of phosphate buffered saline containing $1.25 \%$ formaldehyde, $1 \mathrm{mM}$ $\mathrm{Na}_{3} \mathrm{VO}_{4}, 1 \mathrm{mM}$ PMSF, $50 \mathrm{mM} \mathrm{NaF}, 1 \mathrm{mM}$ benzamidine, $0.5 \mu \mathrm{g} / \mathrm{ml}$ leupeptin, $2 \mu \mathrm{g} / \mathrm{ml}$ aprotinin, $10 \mathrm{mM} \beta$-glycerophosphate, $30 \mathrm{mM}$ p-nitrophenyl phosphate, and $0.1 \mathrm{mM} \mathrm{Na}_{2} \mathrm{MoO}_{4}$, minced, and mixed by rotation for $12 \mathrm{~min}$ at room temperature. The crosslinking reaction was stopped with the addition of glycine to a final concentration of $125 \mathrm{mM}$. Samples were homogenized in a Dounce homogenizer ( 8 strokes, loose pestle), incubated $15 \mathrm{~min}$ on ice, and then centrifuged $5 \mathrm{~min}$ at $1,500 \mathrm{xg}$ at $4^{\circ} \mathrm{C}$. The pellets were resuspended in 3 volumes of cell lysis buffer $(5 \mathrm{mM}$ HEPES, pH 8.0, $85 \mathrm{mM} \mathrm{KCl}, 0.5 \% \mathrm{NP} 40,1 \mathrm{mM} \mathrm{Na}_{3} \mathrm{VO}_{4}, 1 \mathrm{mM}$ PMSF, $50 \mathrm{mM} \mathrm{NaF}, 1 \mathrm{mM}$ benzamidine, $0.5 \mu \mathrm{g} / \mathrm{ml}$ leupeptin, $2 \mu \mathrm{g} / \mathrm{ml}$ aprotinin, $10 \mathrm{mM} \beta$-glycerophosphate, $30 \mathrm{mM} \mathrm{p}$ nitrophenyl phosphate, and $0.1 \mathrm{mM} \mathrm{Na} 2 \mathrm{MoO}_{4}$ ), homogenized in a Dounce homogenizer (15 
strokes, loose pestle), incubated on ice for $15 \mathrm{~min}$, and centrifuged at $3500 \mathrm{xg}$ for $5 \mathrm{~min}$ at $4^{\circ} \mathrm{C}$. The pellets were resuspended in 1 volume of nuclear lysis buffer ( $1 \% \mathrm{SDS}, 50 \mathrm{mM}$ Tris$\mathrm{HCl}, \mathrm{pH}$ 8.1, $10 \mathrm{mM}$ EDTA, and protease and phosphatase inhibitors as listed above), homogenized with a Dounce homogenizer (5 strokes, loose pestle), aliquoted into $1 \mathrm{ml}$ volumes, and incubated on ice for $10 \mathrm{~min}$. Samples were sonicated with continuous pulse in 4 x $15 \mathrm{sec}$ bursts. The probe was chilled between each pulse. Samples were then cleared by centrifugation at $19,000 \times \mathrm{g}$ for $10 \mathrm{~min}$. Supernatants were diluted 1:5 with dilution buffer (0.01\% SDS, 1.1\% Triton X-100, 16.7 mM MOPS, pH 7.3, $1.2 \mathrm{mM}$ EDTA, $167 \mathrm{mM} \mathrm{NaCl}, 1 \mathrm{mM}$ $\mathrm{Na}_{3} \mathrm{VO}_{4}, 1 \mathrm{mM}$ PMSF, $50 \mathrm{mM} \mathrm{NaF}, 1 \mathrm{mM}$ benzamidine, $0.5 \mu \mathrm{g} / \mathrm{ml}$ leupeptin, $2 \mu \mathrm{g} / \mathrm{ml}$ aprotinin, $10 \mathrm{mM} \beta$-glycerophosphate, $30 \mathrm{mM}$ p-nitrophenyl phosphate, and $0.1 \mathrm{mM} \mathrm{Na}_{2} \mathrm{MoO}_{4}$ ), and stored at $-80^{\circ} \mathrm{C}$ until use.

For the immunoprecipitation, protein $A / G$ agarose beads (Santa Cruz) were washed five times in dilution buffer and blocked in dilution buffer containing $5 \mathrm{mg} / \mathrm{ml} \mathrm{BSA}$ and $250 \mu \mathrm{g} / \mathrm{ml}$ sonicated salmon sperm DNA. An aliquot from each sample was removed prior to immunoprecipitation to serve as the "input". The remainder of each sample was pre-cleared with blocked agarose beads for $2 \mathrm{~h}$ with rotation and centrifuged. The supernatants were transferred into fresh tubes to which the following antibodies were added (7.5 to $15 \mu \mathrm{g})$ : phosphorylated SR (1H4, Invitrogen), (heterogeneous nuclear ribonucleoprotein) hnRNP M (Invitrogen), or RNA polymerase II (Covance). The "no antibody" control was treated identically except it received no primary antibody. Samples were immunoprecipitated overnight with rotation at $4^{\circ} \mathrm{C}$, after which samples were rotated with blocked beads for $2 \mathrm{~h}$ and washed once with each of the following buffers: low salt buffer (20 mM MOPS, pH 7.3, $150 \mathrm{mM} \mathrm{NaCl}, 2 \mathrm{mM}$ EDTA, $0.1 \%$ SDS, 1\% Triton X-100), high salt buffer (20 mM MOPS, pH 7.3, $500 \mathrm{mM} \mathrm{NaCl}, 2$ mM EDTA, 0.1\% SDS, 1\% Triton-X 100), LiCl buffer (10 mM MOPS, pH 7.3, 1 mM EDTA, 
$0.25 \mathrm{M} \mathrm{LiCl}, 1 \% \mathrm{NP} 40,1 \%$ deoxycholate), and then washed twice with buffer containing $10 \mathrm{mM}$ MOPS, pH 7.3 and $1 \mathrm{mM}$ EDTA. All wash buffers contained protease and phosphatase inhibitors as listed above. The immunoprecipitated proteins were eluted from the agarose beads with $0.1 \mathrm{M} \mathrm{NaHCO}_{3}$ and $1 \%$ SDS. Immunoprecipitated and input samples were reversecrosslinked with $200 \mathrm{mM} \mathrm{NaCl}$ at $65^{\circ} \mathrm{C}$ for $6 \mathrm{~h}$ and digested with proteinase $\mathrm{K}(180 \mu \mathrm{g} / \mathrm{ml})$ for 1 $\mathrm{h}$ at $45^{\circ} \mathrm{C}$ and the DNA purified. Immunoprecipitated DNA was measured using real-time PCR (QuantiTect probe PCR reagents, Qiagen) and the primers and dual-labeled probes (IDT) listed in supplemental Table 1. The amount of DNA that was immunoprecipitated relative to the amount present in total input chromatin was determined with the following formulas: $\Delta \mathrm{Ct}=\mathrm{Ct}$ (input)-Ct(IP), \% total $=2^{\mathrm{Ct}} \times 1.7$, where $1.7 \%$ is the percent that the input chromatin represents of the total chromatin (22). This value was then normalized to the signal found in the "no antibody" control.

RNA EMSA - The RNA EMSA protocol is a modification of existing methods $(23,24)$. Briefly, 100 fmol of a 5'-hexachlorofluorescein (HEX $\left.{ }^{\text {TM }}\right)$ labeled-RNA probe (IDT) corresponding to the G6PD regulatory element (supplemental Table 1) was mixed with $0.27 \mu \mathrm{g}$ of purified recombinant glutathione S-transferase (GST) conjugated-SRSF3 (Abnova) in 1X binding buffer (10 mM Tris pH 7.5, $1 \mathrm{mM} \mathrm{MgCl} 2,100 \mathrm{mM} \mathrm{KCl}, 0.1 \mathrm{mM}$ DTT, $5 \%$ glycerol; ref. 25) plus/minus unlabeled competitor oligonucleotides $(0,1 \mathrm{pmol}, 5 \mathrm{pmol}, 10 \mathrm{pmol}$; supplemental Table 1; IDT) in a total reaction volume of $20 \mu \mathrm{L}$. Reactions were incubated for $30 \mathrm{~min}$ at room temperature and then loaded onto a pre-running $5 \%$ native polyacrylamide gel. The gel was imaged directly on a Typhoon 9410 Imager and the signals quantified using ImageQuant TL software.

SiRNA transfection - HepG2 (human hepatoma) cells stably expressing an RNA splicing reporter (pß-gal ex12-ex13, supplemental Fig. 2) or HeLa cells were used for knockdown of SR 
proteins. One day after plating, HepG2 cells $\left(5 \times 10^{5}\right)$ or HeLa cells $\left(2 \times 10^{5}\right)$ were transfected with $75 \mathrm{nM}$ siRNA pools using TransIT-siQUEST® Transfection Reagent (Mirus) in MEM containing 10\% FBS. SiGENOME SMARTpools (Dharmacon) consisting of 4 siRNA duplexes (supplemental Table 1) were used for each target mRNA. SiGENOME non-targeting siRNA pool \#1 was used as the negative control. Total RNA and whole-cell protein extracts were collected after $48 \mathrm{~h}$. Cell lysates were prepared using RIPA buffer plus phosphatase and protease inhibitors (Thermo Scientific). HeLa cells were first transfected with the siRNA and then 24 later transiently transfected with the splicing reporter ( $p \beta-g a l$ ex12-ex13) using TransIT®-LT1 Transfection Reagent (Mirus) in complete MEM. Total RNA and whole-cell protein extracts were collected after an additional $24 \mathrm{~h}$.

RNA Immunoprecipitation (RIP) - The RIP protocol is a modification of existing methods $(26,27)$. For HepG2 cells, $5 \times 10^{6}$ cells were crosslinked in serum-free DMEM containing $1 \%$ formaldehyde for $10 \mathrm{~min}$ with agitation. The reaction was stopped by the addition of glycine to a final concentration of $125 \mathrm{mM}$ and incubated for $5 \mathrm{~min}$. The cells were washed twice with icecold 1x PBS and collected in ice-cold RIPA++ buffer (1X RIPA Buffer, 1X Halt ${ }^{\mathrm{TM}}$ Protease and Phosphatase Inhibitor Cocktail and 2U/uL SUPERase $\bullet \mathrm{n}^{\mathrm{TM}}{ }^{\mathrm{R} N A s e}$ Inhibitor; Invitrogen), followed by sonication in a Diagenode BioRuptor (two 15-min cycles, medium intensity, $30 \mathrm{sec}$ on/off pulses). The lysates were cleared by centrifugation and the total protein content of the lysates was determined using the Bradford method (Thermo Scientific). Lysate containing 500ug of protein was suspended in IP buffer (1x PBS, 2X Halt ${ }^{\mathrm{TM}}$ Protease and Phosphatase Inhibitor Cocktail and 2U/uL SUPERase $\bullet \mathrm{I}^{\mathrm{TM}} \mathrm{RNAse}$ Inhibitor) and pre-cleared with protein $\mathrm{G}$ Dynabeads at $4^{\circ} \mathrm{C}$ for $2 \mathrm{~h}$ with rotation. A portion of the lysate was saved for the "input" sample. 
In a separate tube, protein G Dynabeads (Invitrogen) were washed twice with 1x PBS+0.02\% Tween 20 (PBS/T). Antibodies (10-20 $\mu \mathrm{g}$, SRSF3, Invitrogen; RNA Polymerase II, Covance; and Normal Mouse IgG, Millipore) were added to the beads and incubated at room temperature for $1 \mathrm{~h}$ with rotation. The antibody-bound beads were washed three times with $\mathrm{PBS} / \mathrm{T}$ and then mixed with the pre-cleared lysates. The bead/lysate mixtures were incubated overnight at $4^{\circ} \mathrm{C}$ with rotation and then washed six times with PBS/T. RNA bound to the immunoprecipitated proteins was released by digestion with proteinase $\mathrm{K}$. The RNA was extracted with $1 \mathrm{~mL}$ of TriReagent and DNA was removed by DNase I digestion. The G6PD splicing regulatory element was detected in the immunoprecipitated RNA by real-time RT-PCR using the primers indicated in supplemental Table 1. Amplification in the absence of RT was used as a control for chromatin contamination.

In experiments with HepG2 cells, the cells were transiently transfected as described above with FLAG®-tagged SRSF3 (gift of Dr. Sandri-Goldin, 28). The cells were crosslinked 48 h after transfection and SRSF3 was immunoprecipitated with anti- FLAG® antibody (SigmaAldrich).

For detection of SRSF3 binding to the regulatory element in whole liver, 8-10 week old male C57BL/6J (The Jackson Laboratory) were fasted for $18 \mathrm{~h}$ or were fasted for $18 \mathrm{~h}$, and then refed a low-fat, high-carbohydrate diet (Basal Mix TD.00235, Harlan Laboratories; supplemented with $1 \%$ safflower oil) for $12 \mathrm{~h}$. The livers were removed, minced with a razor blade and crosslinked in four volumes of 1 PBS containing $1 \%$ formaldehyde for $10 \mathrm{~min}$ at room temperature and with mild agitation. The reaction was stopped with glycine as previously described. Samples were centrifuged for 4 min at $300 \times g$ at $4^{\circ} \mathrm{C}$ to pellet the crosslinked tissue. The pellets were resuspended in two volumes of ice-cold RIPA++ buffer, homogenized 
using a Dounce homogenizer (15 strokes, loose pestle), followed by sonication. All subsequent steps were as described for HepG2 cells.

Statistical Analyses - For Figs. 1 and 2, one-way ANOVA was performed, and, if the overall $p$-value was significant $(p<0.05)$, Dunnett's Multiple Comparison Test was performed to make pairwise comparisons to the insulin treatment (media alone vs. insulin and insulin vs. insulin + arachidonic acid; GraphPad Prism, version 4.0). All other statistical comparisons were made using Student's t-test. 


\section{RESULTS}

Previous work in our laboratory identified three members of the hnRNP family $(\mathrm{K}, \mathrm{L}$, and A2/B1), whose binding to a splicing regulatory element in the G6PD mRNA increased during starvation and occurred coincident with a decrease in exon 12 splicing (19). In contrast to these inhibitory proteins, enhanced splicing during refeeding could involve SR proteins that bind within exons and are splicing enhances. Because SR proteins undergo regulatory phosphorylation, we hypothesized that this family of proteins may also be targets for regulation by nutritional status. We used 3 approaches to test if SR proteins can be intermediates in the nutritional regulation of splicing: (1) measurement of changes in SR protein activity, (2) changes in binding of SR proteins to an ESE in response to changes in nutritional status, and (3) loss-of-function via siRNA-mediated depletion of candidate SR proteins.

Phosphorylation of SR proteins changes with insulin and arachidonic acid treatment-As a first approach to addressing a physiological role for SR proteins in nutrient-regulated splicing, we asked if the hormones and nutrients involved in regulating gene expression could alter SR protein amount or phosphorylation. Primary rat hepatocytes are the most insulin-responsive liver cell model and regulate their metabolism in response to fatty acids in a manner similar to intact liver $(16,29)$. Treatment of primary rat hepatocytes with insulin and a high-glucose medium reflects the humoral milieu of the refed state and these treatments significantly increased the amount of phosphorylated SRSF1, SRSF2, and SRSF3 as detected with the monoclonal antibody, mAb104 that detects a phosphorylated epitope in these proteins (Figs. $1, A$ and $B)$. The increase in phosphorylated SRSF4 and SRSF6 upon treatment with insulin was not significantly different from untreated hepatocytes, possibly due to variation in the response. In contrast, treatment of hepatocytes with the non-esterified fatty acid, arachidonic acid, significantly decreased the amounts of phosphorylated SRSF3 and SRSF5 by $50-80 \%$. 
Equal amounts of protein were used for the analysis as indicated by the control protein, lamin A/C (Fig. 1A). These changes in the amounts of phosphorylated SR proteins by insulin and fatty acids were coincident with changes in the accumulation of G6PD mRNA (Fig. 1C).

Previous work in our laboratory has demonstrated that changes in G6PD mRNA by insulin and arachidonic acid are solely due to changes in splicing of the primary transcript and mediated through a splicing regulatory element in exon 12 of the mRNA $(2,16)$. Thus, changes in the amount of phosphorylated SR proteins may play a role in regulated splicing.

The detected changes in SR protein phosphorylation could reflect either a change in the phosphate content of the proteins or a change in the total amount of the protein. To measure the amounts of total SR proteins we used an antibody $(16 \mathrm{H} 3)$ that detects the SRSF proteins 3 , 4, 5 and 6 regardless of phosphorylation state. Incubation of hepatocytes with insulin resulted in 1.2 fold increase in the abundance of SRSF3 and SRSF6, a 2-fold increase in SRSF5, and little or no change in the amount of SRSF4 (Fig. 1,D and E). The addition of arachidonic acid to the hepatocytes decreased the abundance of SRSF3, SRSF5 and SRSF6 by $50 \%$ or more, but had little or no effect on the abundance of SRSF4. Thus the changes in phosphorylated SR protein amounts are caused in part by changes in the abundance of these proteins within the nucleus.

Refeeding increases the binding of SR proteins to the splicing regulatory element-As a second approach to test if SR proteins are involved in nutrient-regulated splicing, we measured SR protein binding to an ESE in the livers of mice that were starved or refed. Binding was measured using ChIP analysis, which can detect RNA-protein interactions because splicing occurs co-transcriptionally resulting in close proximity between splicing factors bound to the newly transcribed RNA and chromatin $(5,30)$. When subjected to crosslinking agents, the RNAbound splicing factors become covalently attached to the chromatin adjacent to the nascent 
RNA transcript (31). Exon 12 of G6PD mRNA contains a regulatory region that has ESE activity and is involved in mediating the effects of insulin and arachidonic acid on G6PD mRNA splicing (2). As previously demonstrated and diagrammed in Fig. 2A, silenced splicing of the G6PD transcript results in intron retention surrounding exon 12 (15).

Starvation and refeeding cause the greatest changes in G6PD mRNA accumulation (7fold or more, 13). Immunoprecipitation with a monoclonal antibody that recognizes phosphorylated RS domains allowed detection of binding by most members of the SR protein family. Refeeding significantly increased the binding of phosphorylated SR proteins to the splicing regulatory element by 1.8 -fold above the fasting level (Fig. $2 B$ ). To control for potential cross-reactivity between this antibody and the phosphorylated C-terminal domain of RNA polymerase II $(32,33)$, we performed a second immunoprecipitation with an antibody against Pol II. Dietary treatment did not alter the occupancy of Pol II across this region of exon 12 , indicating that an increase in SR protein binding was occurring (Fig. 2B). The lack of change in Pol II occupancy on the G6PD chromatin is consistent with the absence of transcriptional regulation of this gene (14). HnRNP M immunoprecipitation was performed as a negative control because previous experiments have determined that this RNA-binding protein does not bind to the splicing regulatory element in vitro (2). As expected, binding of hnRNP M to the regulatory element was low, and this binding was not altered by the nutritional status of the animal (Fig. 2B). Refeeding did not cause a generalized increase in splicing factor binding to newly transcribed mRNA as SR protein binding to phosphoenolpyruvate carboxykinase (PEPCK) mRNA was not increased by refeeding (Fig. 2C). These data are the first to demonstrate regulation of SR protein binding to an RNA element in the whole animal. SRSF3 and 4 bind to the G6PD ESE-The ChIP assay can not discriminate between SR proteins bound to RNA polymerase II and SR proteins bound to the RNA. Thus, an RNA affinity 
assay was used to verify that SR proteins bind to the RNA and also to determine which SR proteins were bound. RNA oligonucleotides corresponding to the splicing regulatory element (nucleotides 43-72 of G6PD exon 12; Fig. 2A) were attached to agarose beads and used to affinity-purify RNA-binding proteins from rat liver nuclear extracts. An RNA oligonucleotide corresponding to a region of exon 12 outside of the regulatory element (control region; nucleotides 79-93) was used to detect non-specific binding of proteins to RNA, while the beads alone were included to detect background binding (Fig. 3). Liver nuclear extracts contained SRSF3, SRSF4, SRSF5 and SRSF6 (Fig. 3A). The additional bands in upper panel are not unexpected, as the $16 \mathrm{H} 3$ antibody will detect other proteins with SR repeats (33). SRSF3 was detected in a second immunoblot using more nuclear protein, as it is more difficult to detect with this antibody presumably due to its lower abundance in liver compared to other SR proteins. Following pull-down with the splicing regulatory element, a band the size of SRSF4 was detected (Fig. 3B). The band near $37 \mathrm{kDa}$ is not SRSF1 or 2, which migrate at this size, because the $16 \mathrm{H} 3$ antibody does not detect these SR proteins. To verify the identities of the bound proteins, the blots were re-probed with a different antibody $(1 \mathrm{H} 4)$, which detects phosphorylated forms of the SR proteins including SRSF1 and SRSF2. SRSF4 binding was also detected with this antibody but the band near $37 \mathrm{kDa}$ was not detected (data not shown). In addition, SRSF3 binding to the regulatory element was also observed (Fig. $3 C$ ). An SRSF3specific antibody was used for detection to better visualize this protein. Neither SR protein bound to the control RNA oligonucleotide or the beads alone. Thus, SRSF3 and SRSF4 are candidate SR proteins involved in regulating splicing of the G6PD mRNA.

Purified SRSF3 binds to the G6PD regulatory element- RNA EMSA was used to confirm the binding of SRSF3 to the regulatory element. Purified SRSF3 conjugated to GST was incubated with an RNA oligonucleotide representing the 30 nt regulatory element in exon 12 
(Fig. 4,A and B). This complex resulted in a single shifted band (Fig. $4 C$ ). The shifted band was competed to a maximum of $10 \%$ by increasing concentrations of the unlabeled wild type RNA (Fig. 4C). We next tested the ability of the 3 mutated sequences to compete for SRSF3 binding (Fig. 4A). These mutations represent the same sequence changes previously used by us that abrogated the inhibition of splicing by arachidonic acid using splicing reporter constructs in functional assays in rat hepatocytes (2). Mutation of the first $10 \mathrm{nt}$ of this sequence resulted in an RNA that competed for SRSF3 binding as effectively as the wild type sequence. Mutation of the second $10 \mathrm{nt}$ attenuated the competition to only $33 \%$ of the wild type. Mutation of the final $10 \mathrm{nt}$ resulted in little to no competition for SRSF3 binding (70\% at the highest concentration of competitor). Thus, SRSF3 can bind to the splicing regulatory element and appears to bind to the latter half of this element.

SiRNA depletion of SRSF3 decreases G6PD mRNA accumulation-To test the functional significance of SR proteins in regulating splicing of G6PD mRNA in cells, we used a loss-offunction approach. SiRNA-mediated knockdowns of SRSF3 and SRSF4 were performed in a clonal isolate of HepG2 cells stably expressing a G6PD splicing reporter (Fig. 5A). HepG2 cells were used as a liver cell model because they can be transfected with a higher efficiency than primary rat hepatocytes. The splicing reporter, p $\beta$-gal ex12-ex13 (Fig. 5A), contains exon 12, intron 12, exon 13, and the 3' UTR of G6PD ligated to the $\beta$-galactosidase gene. This construct retains splicing regulation by insulin and fatty acid (2) and permits evaluation of SR protein activity involving the exon 12 ESE without interference from regulatory elements in other exons of the G6PD pre-mRNA. Knockdowns of SRSF3 and SRSF4 were $80 \%$ and 70\%, respectively, across 3 separate experiments (Fig. 5,B and $C$ ). In each case, knockdown of the specific SR protein did not result in a significant difference in the expression of other members of the SR protein family or the control proteins lamin A/C (Fig. 5B and data not shown). 
Knockdown to greater than $80 \%$ could be achieved but resulted in decreased expression of the other SR proteins. The $80 \%$ decrease in SRSF3 amount was accompanied by a significant inhibition (60\%) of the expression of the G6PD reporter RNA in the absence of an effect on cyclophilin B mRNA, an endogenous control (Fig. 5D, left 2 graphs). The decrease in G6PD reporter expression represented a decrease in the amount of spliced RNA for this reporter (Fig. $5 D$, bottom right). In addition, a significant decrease was also observed in the expression of the endogenous G6PD mRNA (Fig. 5D, top right). In contrast, depletion of SRSF4 did not inhibit expression of the splicing reporter or the endogenous G6PD gene (Fig. 5D).

To test for cell-type-specific effects of knockdown, we employed a similar strategy in HeLa cells transiently expressing the reporter construct (Fig. $5 A$ ). Depletion of SRSF3 by $80 \%$ or more (Fig. 6,A and $B$ ) resulted in significant decreases in accumulation of RNA from both the transiently transfected splicing reporter and the endogenous G6PD gene (Fig. 6C). Accumulation of the reporter mRNA was inhibited by $44 \%$ and accumulation of mRNA for the endogenous G6PD gene was inhibited by $30 \%$ in comparison to cells transfected with nontargeting (NT) siRNA. These decreases are physiologically significant since the total elimination of G6PD expression causes embryonic lethality (34). Similar to the results with HepG2 cells, knockdown of SRSF4 by $90 \%$ or more (Fig. $6, A$ and $B$ ) did not inhibit accumulation of either the reporter or the endogenous G6PD mRNAs and, in fact, increased expression 1.9-fold compared to NT siRNA (Fig. 6C). As a negative control, siRNA against SRSF6 was used because SRSF6 does not bind to the exon 12 regulatory element (Fig. 3B). SiRNA treatment depleted SRSF6 by $94 \%$ compared to the NT siRNA but this decrease did not inhibit expression of the splicing reporter RNA (Fig. 6). A small decrease in endogenous G6PD expression was observed in these cells upon SRSF6 knockdown, which may reflect a potential interaction with other exons within the G6PD transcript (Fig 6C). As in the HepG2 cell, 
knockdown of each SR protein did not significantly impact the expression of the other SR proteins (Fig. $6 \mathrm{~A}$ and data not shown). Together, these data demonstrate that SRSF3 is involved in splicing of G6PD RNA across multiple cells types.

Refeeding enhances binding of SRSF3 to the splicing regulatory element in vivo - To verify that SRSF3 binds to the splicing regulatory element in intact cells, we used RIP and primers within exon 12 that amplify the region containing the regulatory element. In HepG2 cells, a 6-fold enrichment of SRSF3 binding was observed as compared to immunoprecipitation using the isotype control, IgG (Fig. 7A). To verify that the detected binding was not due to cross-reactivity of the antibody with other SR proteins, HepG2 cells were transiently transfected with SRSF3 containing a FLAG®-tag (Fig. 7B). When RIP was performed with an anti- FLAG® antibody, SRSF3 binding to the splicing regulatory element increased 13- and 7.5-fold compared to immunoprecipitation with IgG in experiments 1 and 2 , respectively (Fig. $7 B)$.

The ability of nutritional status to regulate binding of SRSF3 to the G6PD regulatory element was measured using RIP in livers of mice that were starved or starved and then refed following formaldehyde cross-linking (Fig. 7C). SRSF3 was present in 6-fold greater abundance on the regulatory element in the refed livers as compared to lgG; furthermore, SRSF3 binding was enhanced by 6 -fold or more in refed livers as compared to its binding to the regulatory element in the livers of fasted mice. This dietary paradigm causes a 7-fold or more increase in G6PD mRNA abundance (13) and a similar fold increase in the splicing of exon 12 (15). To the best of our knowledge, these are the first data to demonstrate that nutritional status regulates the presence of an SR protein on a splicing regulatory element and that this binding alters the splicing of the transcript. 


\section{DISCUSSION}

Understanding how nutrients and hormones affect the splicing process introduces a new paradigm for regulating cellular function. Because the majority of genes undergo alternative splicing, this process is central in determining the protein composition of cells. G6PD was the first gene identified in which nutritional status regulates its expression by alternative splicing, specifically, intron retention $(1,2)$. In this report, we demonstrate that SR proteins are targets for the actions of insulin and the polyunsaturated fatty acid arachidonic acid, resulting in changes in their amount and phosphorylation state, and enhanced presence at an ESE within the G6PD gene during active transcription. Of the SR proteins, SRSF3 is playing a specific role in the regulation of G6PD mRNA splicing. A specific role of this protein in nutrient-regulated splicing is supported by the specific binding of SRSF3 to the splicing regulatory element in the G6PD mRNA, the decrease in G6PD RNA splicing upon SRSF3 depletion, and the regulated binding of SRSF3 to the G6PD regulatory element in the livers of starved and refed mice.

SRSF3 functions in both alternative splicing and mRNA transport to the cytosol $(35,36)$.

Our data suggest that SRSF3 is regulating splicing of G6PD mRNA rather than transport because loss of SRSF3 decreased the accumulation of spliced RNA (Figs. 5 and 6). The binding of SR proteins to exon 12 as detected using ChIP (Fig. 2), requires that the proteins be present during active transcription of the RNA and possibly associated with the C-terminal domain of RNA polymerase II. While the ChIP data does not specifically identify which SR proteins are present, the in vitro binding data (Figs. 3 and 4) indicate that only SRSF3 and SRSF4 bind to exon 12.

SRSF3 clearly binds to the regulatory element both in vitro and in intact cells and tissues; however, this element does not contain a canonical SRSF3 binding site. SRSF3 binding sites that have been identified using SELEX or CLIP-seq are CU- and CA-rich 
sequences $(37,38)$. Putative SRSF3 binding sites conforming to these known consensus sequences occur just 5'of the regulatory element and overlapping the 3' end of the element, respectively. Results using RNA EMSA support the binding of SRSF3 to the 3' end of the regulatory element (Fig. 4). While the EMSA data confirm that SRSF3 can bind the regulatory element, in vivo, SRSF3 may be interacting with another RNA-binding protein. The chemical crosslinking used in the RIP analysis permits both protein:RNA and protein:protein crosslinks. UV crosslinking could discriminate between these possibilities but cannot be applied to intact tissue. Nonetheless, sequences are present within G6PD exon 12 and/or the regulatory element that support SRSF3 recruitment.

Most remarkable is the large change in SRSF3 binding with refeeding. This is the first demonstration of regulated binding of SR proteins to mRNA in response to nutritional status. While both SRSF3 and SRSF4 bound to the splicing regulatory element in vitro, only the binding of SRSF3 was relevant for increased splicing of G6PD mRNA. In this regard, knockdown of SRSF4 failed to decrease expression of G6PD mRNA (Figs. 5 and 6). This does not completely rule out involvement of SRSF4 as its loss could have been compensated for by the closely related SRSF6 (39); however, knockdown of SRSF6 did not decrease G6PD RNA accumulation. The involvement of SRSF3 in the nutritional regulation of splicing is further supported by the regulation of SRSF3 phosphorylation and amount in response to insulin and arachidonic acid. Upon phosphorylation, SR proteins move from the cytoplasm to the nucleus, and from sites of concentration within the nucleus, called speckles, to nascent RNA at transcription sites (8). In addition, phosphorylation enhances binding to RNA regulatory elements and protein-protein interactions $(40,41)$. SR proteins bound to the G6PD regulatory element during transcription were phosphorylated based on their detection with a phosphorylation-specific antibody in ChIP analysis (Fig. 2). The increase in binding of SR 
proteins during refeeding is in contrast with our earlier data demonstrating an opposite pattern of binding by proteins that silence splicing. In this regard, members of the hnRNP family of splicing silencing proteins, $K, \mathrm{~L}$ and $\mathrm{A} 2 / \mathrm{B} 1$, bind to the G6PD regulatory element in the starved state, and this binding is decreased by refeeding (19). Competition between SRSF3 and splicing silencers has been demonstrated in regulation of the alternative splicing of the insulin receptor (36). A similar competition for binding may be part of the regulatory mechanism controlling G6PD splicing in response to nutrients and nutritional status.

External stimuli and hormones can exert an effect upon splicing by increasing or decreasing the expression of splicing factors and/or by changing their activity $(9,10,42,43)$. A considerable body of literature supports a broad role of insulin in alternative splicing. Results from splicing-sensitive microarrays performed with insect cells indicated that greater than $\mathbf{1 5 0}$ genes undergo alternative splicing in response to insulin treatment (44). In mammalian cells, insulin regulates alternative splicing of $\operatorname{PKC} \beta \| \mathrm{II}(45,46)$. The effect of insulin upon alternative splicing of PKC $\beta I$ I is coincident with an increase in the phosphorylation of SRSF5 (11). In hepatocytes in culture, insulin increases G6PD mRNA and increases SR protein phosphorylation, including phosphorylation of SRSF3 (Fig. 1). The coincident increase in G6PD splicing and SRSF3 binding with refeeding likely involves a regulatory role for insulin because insulin is required for the diet-mediated increases in G6PD expression (1).

Previous evidence from our lab demonstrates that the increase in splicing of G6PD that we observe with insulin treatment is due to an increase in its signaling through the PI3K pathway; whereas arachidonic acid treatment attenuates insulin signaling by inhibiting PI3K activity (47). Arachidonic acid decreases the amount of phosphorylated SR proteins and causes a coincident decrease in the accumulation of G6PD mRNA (Fig. 1). The presence of a consensus sequence for Akt phosphorylation in the RS domain of SRSF3 suggests that this 
protein can be a target of insulin action directly. Insulin action could also be indirect via stimulation of the SR protein kinase, Clk/Sty; which is known to phosphorylated upon insulin treatment (10). Consistent with this concept, the phosphorylation-specific antibody used in these studies detects phosphorylations by multiple kinases including Clk/Sty. Identifying the relevant kinases involved in SR protein phosphorylation in intact liver and primary rat hepatocyte cultures is the subject of ongoing investigations in the laboratory. The decrease in phosphorylation of SR proteins with arachidonic acid treatment may be the result of reduced insulin signal transduction, or alternatively, may be due to an increase in phosphatase activity. The bioactive lipid, ceramide, reduces phosphorylation of SR proteins via activation of protein phosphatase 1 (48), and arachidonic acid has been shown to induce ceramide production from sphingomyelin in HL-60 cells (49).

The finding that insulin, arachidonic acid and nutritional status regulate the phosphorylation and/or amount of the SR proteins has consequences beyond the regulation of G6PD expression. SR proteins enhance the splicing of multiple genes; thus, nutritional status could impact the splicing of many transcripts in addition to G6PD. In this regard, splicing of the insulin receptor mRNA is also regulated by SRSF3 and the resulting isoform switch may be involved in liver development and/or liver regeneration (36). Increased SRSF3 abundance in tumors and cancer cells, and identification of multiple SRSF3 targets in differentiating neural cells highlight a much broader role of this protein in organisms (50).

In summary, these data indicate that nutrients and nutritional status induce changes in the activity of SR proteins, which impacts the splicing of a metabolic gene. Nutrient-regulated splicing provides another layer of control over gene expression beyond the well-described effects upon transcription (29). We hypothesize that the RNA transcripts of other metabolic genes are regulated by nutrients at the step of splicing. Fully understanding the process by 
which our metabolic organs regulate splicing in response to nutrients and hormones will provide a new paradigm for nutrient regulation of cellular function.

\section{ACKNOWLEDGEMENTS}

We thank Dr. Roz Sandri-Goldin (University of California, Irvine) for the gift of the plasmid expressing the FLAG®-tagged SRSF3. We thank Peter Stoilov for advice and critically reading the manuscript. We also thank Matthew Myers for his assistance with the conduct of experiments in figure 4. 


\section{REFERENCES}

1. Salati, L. M., Szeszel-Fedorowicz, W., Tao, H., Gibson, M. A., Amir-Ahmady, B., Stabile, L. P., and Hodge, D. L. (2004) Nutritional regulation of mRNA processing. $J$ Nutr 134, 2437S-2443S

2. Szeszel-Fedorowicz, W., Talukdar, I., Griffith, B. N., Walsh, C. M., and Salati, L. M. (2006) An exonic splicing silencer is involved in the regulated splicing of glucose 6phosphate dehydrogenase mRNA. J Biol Chem 281, 34146-34158

3. Kalsotra, A., and Cooper, T. A. (2011) Functional consequences of developmentally regulated alternative splicing. Nat Rev Genet 12, 715-729

4. Stamm, S. (2008) Regulation of alternative splicing by reversible protein phosphorylation. J Biol Chem 283, 1223-1227

5. Pan, Q., Shai, O., Lee, L. J., Frey, B. J., and Blencowe, B. J. (2008) Deep surveying of alternative splicing complexity in the human transcriptome by high-throughput sequencing. Nat Genet 40, 1413-1415

6. Long, J. C., and Caceres, J. F. (2009) The SR protein family of splicing factors: master regulators of gene expression. Biochem J 417, 15-27

7. Zhu, J., Mayeda, A., and Krainer, A. R. (2001) Exon identity established through differential antagonism between exonic splicing silencer-bound hnRNP A1 and enhancer-bound SR proteins. Mol Cell 8, 1351-1361

8. Lin, S., and Fu, X. D. (2007) SR proteins and related factors in alternative splicing. Adv Exp Med Biol 623, 107-122

9. Blaustein, M., Pelisch, F., Tanos, T., Munoz, M. J., Wengier, D., Quadrana, L., Sanford, J. R., Muschietti, J. P., Kornblihtt, A. R., Caceres, J. F., Coso, O. A., and Srebrow, A. 
(2005) Concerted regulation of nuclear and cytoplasmic activities of SR proteins by AKT. Nat Struct Mol Biol 12, 1037-1044

10. Jiang, K., Patel, N. A., Watson, J. E., Apostolatos, H., Kleiman, E., Hanson, O., Hagiwara, M., and Cooper, D. R. (2009) Akt2 regulation of Cdc2-like kinases (Clk/Sty), serine/arginine-rich (SR) protein phosphorylation, and insulin-induced alternative splicing of PKCbetall messenger ribonucleic acid. Endocrinology 150, 2087-2097

11. Patel, N. A., Kaneko, S., Apostolatos, H. S., Bae, S. S., Watson, J. E., Davidowitz, K., Chappell, D. S., Birnbaum, M. J., Cheng, J. Q., and Cooper, D. R. (2005) Molecular and genetic studies imply Akt-mediated signaling promotes protein kinase Cbetall alternative splicing via phosphorylation of serine/arginine-rich splicing factor SRp40. J Biol Chem 280, 14302-14309

12. Rognstad, R. (1979) Rate-limiting steps in metabolic pathways. J Biol Chem 254, 18751878

13. Amir-Ahmady, B., and Salati, L. M. (2001) Regulation of the processing of glucose-6phosphate dehydrogenase mRNA by nutritional status. J Biol Chem 276, 10514-10523

14. Stabile, L. P., Hodge, D. L., Klautky, S. A., and Salati, L. M. (1996) Posttranscriptional regulation of glucose-6-phosphate dehydrogenase by dietary polyunsaturated fat. Arch Biochem Biophys 332, 269-279

15. Tao, H., Szeszel-Fedorowicz, W., Amir-Ahmady, B., Gibson, M. A., Stabile, L. P., and Salati, L. M. (2002) Inhibition of the splicing of glucose-6-phosphate dehydrogenase precursor mRNA by polyunsaturated fatty acids. J Biol Chem 277, 31270-31278

16. Stabile, L. P., Klautky, S. A., Minor, S. M., and Salati, L. M. (1998) Polyunsaturated fatty acids inhibit the expression of the glucose-6-phosphate dehydrogenase gene in 
primary rat hepatocytes by a nuclear posttranscriptional mechanism. J Lipid Res 39, 1951-1963

17. Dignam, J. D., Lebovitz, R. M., and Roeder, R. G. (1983) Accurate transcription initiation by RNA polymerase II in a soluble extract from isolated mammalian nuclei. Nucleic Acids Res 11, 1475-1489

18. Schibler, U., Hagenbuchle, O., Wellauer, P. K., and Pittet, A. C. (1983) Two promoters of different strengths control the transcription of the mouse alpha-amylase gene Amy1a in the parotid gland and the liver. Cell 33, 501-508

19. Griffith, B. N., Walsh, C. M., Szeszel-Fedorowicz, W., Timperman, A. T., and Salati, L. M. (2006) Identification of hnRNPs K, L and A2/B1 as candidate proteins involved in the nutritional regulation of mRNA splicing. Biochim Biophys Acta 1759, 552-561

20. Friedman, J. R., Larris, B., Le, P. P., Peiris, T. H., Arsenlis, A., Schug, J., Tobias, J. W., Kaestner, K. H., and Greenbaum, L. E. (2004) Orthogonal analysis of C/EBPbeta targets in vivo during liver proliferation. Proc Natl Acad Sci U S A 101, 12986-12991

21. Yin, L., Wang, Y., Dridi, S., Vinson, C., and Hillgartner, F. B. (2005) Role of CCAAT/enhancer-binding protein, histone acetylation, and coactivator recruitment in the regulation of malic enzyme transcription by thyroid hormone. Mol Cell Endocrinol 245, 43-52

22. Frank, S. R., Schroeder, M., Fernandez, P., Taubert, S., and Amati, B. (2001) Binding of c-Myc to chromatin mediates mitogen-induced acetylation of histone $\mathrm{H} 4$ and gene activation. Genes Dev 15, 2069-2082

23. Hellman, L. M., and Fried, M. G. (2007) Electrophoretic mobility shift assay (EMSA) for detecting protein-nucleic acid interactions. Nat Protoc 2, 1849-1861 
24. Motta-Mena, L. B., Smith, S. A., Mallory, M. J., Jackson, J., Wang, J., and Lynch, K. W. (2011) A disease-associated polymorphism alters splicing of the human CD45 phosphatase gene by disrupting combinatorial repression by heterogeneous nuclear ribonucleoproteins (hnRNPs). J Biol Chem 286, 20043-20053

25. Setzer, D. R. (1999) Measuring equilibrium and kinetic constants using gel retardation assays. Methods Mol Biol 118, 115-128

26. Selth, L. A., Gilbert, C., and Svejstrup, J. Q. (2009) RNA immunoprecipitation to determine RNA-protein associations in vivo. Cold Spring Harb Protoc 2009, pdb prot5234

27. Ule, J., Jensen, K., Mele, A., and Darnell, R. B. (2005) CLIP: a method for identifying protein-RNA interaction sites in living cells. Methods 37, 376-386

28. Sciabica, K. S., Dai, Q. J., and Sandri-Goldin, R. M. (2003) ICP27 interacts with SRPK1 to mediate HSV splicing inhibition by altering SR protein phosphorylation. EMBO J 22, $1608-1619$

29. Hillgartner, F. B., Salati, L. M., and Goodridge, A. G. (1995) Physiological and molecular mechanisms involved in nutritional regulation of fatty acid synthesis. Physiol $\operatorname{Rev} 75,47-76$

30. Kornblihtt, A. R. (2007) Coupling transcription and alternative splicing. Adv Exp Med Biol 623, 175-189

31. Listerman, I., Sapra, A. K., and Neugebauer, K. M. (2006) Cotranscriptional coupling of splicing factor recruitment and precursor messenger RNA splicing in mammalian cells. Nat Struct Mol Biol 13, 815-822 
32. Doyle, O., Corden, J. L., Murphy, C., and Gall, J. G. (2002) The distribution of RNA polymerase II largest subunit (RPB1) in the Xenopus germinal vesicle. J Struct Biol $140,154-166$

33. Neugebauer, K. M., Stolk, J. A., and Roth, M. B. (1995) A conserved epitope on a subset of SR proteins defines a larger family of Pre-mRNA splicing factors. J Cell Biol 129, 899-908

34. Longo, L., Vanegas, O. C., Patel, M., Rosti, V., Li, H., Waka, J., Merghoub, T., Pandolfi, P. P., Notaro, R., Manova, K., and Luzzatto, L. (2002) Maternally transmitted severe glucose 6-phosphate dehydrogenase deficiency is an embryonic lethal. $E M B O \mathrm{~J} \mathbf{2 1}$, 4229-4239

35. Huang, Y., and Steitz, J. A. (2001) Splicing factors SRp20 and 9G8 promote the nucleocytoplasmic export of mRNA. Mol Cell 7, 899-905

36. Sen, S., Talukdar, I., and Webster, N. J. (2009) SRp20 and CUG-BP1 modulate insulin receptor exon 11 alternative splicing. Mol Cell Biol 29, 871-880

37. Anko, M. L., Muller-McNicoll, M., Brandl, H., Curk, T., Gorup, C., Henry, I., Ule, J., and Neugebauer, K. M. (2012) The RNA-binding landscapes of two SR proteins reveal unique functions and binding to diverse RNA classes. Genome Biol 13, R17

38. Schaal, T. D., and Maniatis, T. (1999) Selection and characterization of pre-mRNA splicing enhancers: identification of novel SR protein-specific enhancer sequences. Mol Cell Biol 19, 1705-1719

39. Longman, D., Johnstone, I. L., and Caceres, J. F. (2000) Functional characterization of SR and SR-related genes in Caenorhabditis elegans. EMBO J 19, 1625-1637 
40. Xiao, S. H., and Manley, J. L. (1997) Phosphorylation of the ASF/SF2 RS domain affects both protein-protein and protein-RNA interactions and is necessary for splicing. Genes Dev 11, 334-344

41. Yeakley, J. M., Tronchere, H., Olesen, J., Dyck, J. A., Wang, H. Y., and Fu, X. D. (1999) Phosphorylation regulates in vivo interaction and molecular targeting of serine/arginine-rich pre-mRNA splicing factors. J Cell Biol 145, 447-455

42. Massiello, A., Roesser, J. R., and Chalfant, C. E. (2006) SAP155 Binds to ceramideresponsive RNA cis-element 1 and regulates the alternative 5' splice site selection of Bcl-x pre-mRNA. FASEB J 20, 1680-1682

43. Sumanasekera, C., Watt, D. S., and Stamm, S. (2008) Substances that can change alternative splice-site selection. Biochem Soc Trans 36, 483-490

44. Hartmann, B., Castelo, R., Blanchette, M., Boue, S., Rio, D. C., and Valcarcel, J. (2009) Global analysis of alternative splicing regulation by insulin and wingless signaling in Drosophila cells. Genome Biol 10, R11

45. Apostolatos, A., Song, S., Acosta, S., Peart, M., Watson, J. E., Bickford, P., Cooper, D. R., and Patel, N. A. (2012) Insulin promotes neuronal survival via the alternatively spliced protein kinase Cdeltall isoform. J Biol Chem 287, 9299-9310

46. Chalfant, C. E., Watson, J. E., Bisnauth, L. D., Kang, J. B., Patel, N., Obeid, L. M., Eichler, D. C., and Cooper, D. R. (1998) Insulin regulates protein kinase Cbetall expression through enhanced exon inclusion in L6 skeletal muscle cells. A novel mechanism of insulin- and insulin-like growth factor-i-induced 5 ' splice site selection. $J$ Biol Chem 273, 910-916 
47. Talukdar, I., Szeszel-Fedorowicz, W., and Salati, L. M. (2005) Arachidonic acid inhibits the insulin induction of glucose-6-phosphate dehydrogenase via p38 MAP kinase. $J$ Biol Chem 280, 40660-40667

48. Jenkins, G. M., Cowart, L. A., Signorelli, P., Pettus, B. J., Chalfant, C. E., and Hannun, Y. A. (2002) Acute activation of de novo sphingolipid biosynthesis upon heat shock causes an accumulation of ceramide and subsequent dephosphorylation of SR proteins. J Biol Chem 277, 42572-42578

49. Jayadev, S., Linardic, C. M., and Hannun, Y. A. (1994) Identification of arachidonic acid as a mediator of sphingomyelin hydrolysis in response to tumor necrosis factor alpha. J Biol Chem 269, 5757-5763

50. Anko, M. L., Morales, L., Henry, I., Beyer, A., and Neugebauer, K. M. (2010) Global analysis reveals SRp20- and SRp75-specific mRNPs in cycling and neural cells. Nat Struct Mol Biol 17, 962-970 


\section{FIGURE LEGENDS}

Figure 1. Insulin and arachidonic acid regulate the amount of phosphorylated SR proteins in the nuclei of primary rat hepatocytes. Primary rat hepatocytes were incubated in high glucose (27.5 mM) medium alone (NA), insulin (I; $80 \mathrm{mM})$, or insulin plus arachidonic acid (I+AA; 175 $\mu \mathrm{M})$. After $24 \mathrm{~h}$, nuclear extract was prepared and analyzed by western blotting. (A) A representative immunoblot using an antibody against phosphorylated SR proteins (mAB104) and an antibody against lamin $A / C$ is shown. The identities of the SR proteins are listed on the left side of the gel. The "<" symbol indicates the dye front of the gel. (B) Quantitation of the immunoblot data from $n=4$ independent hepatocyte isolations. The amounts of the phosphorylated SR proteins in each treatment were measured by densitometry. (C) Total RNA was isolated from the hepatocytes after $24 \mathrm{~h}$ with the indicated treatments and the amount of G6PD mRNA was measured by real-time RT-PCR. The value for the amount of G6PD mRNA with insulin treatment $(1.5 \pm 0.3 ; n=3)$ was set at 1 and the values for the NA and I+AA treatments are expressed relative to the insulin treatment. (D) Representative immunoblots using antibodies against total SR proteins (16H3; to detect SRSF4, SRSF5 and SRSF6 and SRSF3), or lamin A/C are shown. A second panel shows SRSF4 at a lighter exposure. The identities of the detected proteins are listed on the right side of the gels. (E) Quantitation of the immunoblot data from $n=3$ independent experiments. The amounts of the SR proteins in each treatment were measured by densitometry. Columns with different letters are significantly different $(p<0.05)$.

Figure 2. Refeeding increases the binding of phosphorylated SR proteins to the regulatory element of G6PD exon 12 in vivo. ChIP was performed in livers of mice that were starved for 
$16 \mathrm{~h}$ or starved and then refed a high-carbohydrate diet for $12 \mathrm{~h}$. (A) Scheme of the G6PD RNA containing the regulatory element and observed changes in splicing. The arrows denote the location of the primers. The forward primer ends 6 nt upstream of the 3' splice site of intron 11 and the reverse primer starts 3 nt downstream of the 5 ' splice site in intron 12. (B) Immunoprecipitation used antibodies that detect: phosphorylated-SR protein (phospho-SR), hnRNP M, and RNA polymerase II (Pol II). The phospho-SR antibody (1H4) detects SRSF1, SRSF2, SRSF3, SRSF4, SRSF5, and SRSF6. The immunoprecipitated DNA was measured using real-time PCR and primers adjacent to exon $12(\mathrm{~A})$. The amount of DNA detected is expressed relative to the input chromatin as described in experimental procedures. The "*" symbol indicates $p<0.05, n=3$ mice. $(C)$ Detection of PEPCK in the immunoprecipitated samples. PEPCK DNA was measured using real-time PCR and primers to exon 2 ( $n=2-3$ mice).

Figure 3. SRSF3 and SRSF4 bind to the splicing regulatory element. An RNA affinity assay was performed using liver nuclear extracts ( $250 \mu \mathrm{g}$ of protein) and RNA oligonucleotide-bead complexes representing the regulatory element (nucleotides 43-72 of exon 12 of G6PD), a control region (nucleotides $79-93$ of exon 12 of G6PD), or with the beads alone as a control for non-specific binding to the beads. (A) The nuclear extracts used in the affinity assay were analyzed by western analysis with an antibody $(16 \mathrm{H} 3, \mathrm{Pan} S \mathrm{~S})$ that detects most SR proteins independent of phosphorylation state. Due to its lower abundance in liver nuclear extracts, SRSF3 was detected in a second blot using more nuclear protein. Proteins eluted from the bead/oligonucleotide complexes were detected by western analysis using (B) the Pan SR antibody or $(\mathrm{C})$ an antibody against SRSF3. The protein marker $(\mathrm{kDa})$ is indicated to the left of each set of gels. *Refers to a non-specific band that was not detected when the blot was 
re-probed with the phosphorylated SR protein-specific antibody (1H4; not shown). Each RNA affinity assay is a representative of $n=3$ experiments performed using two independent nuclear extract preparations; all experiments showed the same results.

Figure 4. Purified SRSF3 binds to the regulatory element in exon 12. RNA EMSA was performed using a fluorescently labeled probe representing the regulatory element in exon 12. (A) The sequence of the regulatory element and the mutant competitors (Mut1, Mut2, and Mut3). The box denotes the mutated sequence. (B) Purified recombinant GST-tagged SRSF3 (270 ng) visualized by western analysis using an SRSF3 specific antibody. The GST tag shifts the apparent molecular weight to $55 \mathrm{kD}$. (C) EMSA. Each reaction contained $270 \mathrm{ng}$ of SRSF3 and $100 \mathrm{fmol}$ probe. Competitions used 10X, 50X and 100X molar excess of competitor RNA. Wt, refers to the unlabeled probe.

Figure 5. SiRNA-mediated depletion of SRSF3 reduces the splicing of a G6PD reporter and the endogenous G6PD mRNA. HepG2 cells stably expressing a splicing reporter were transiently transfected with siRNA pools directed against SRSF3, SRSF4, or with a nontargeting control siRNA (NT) for $48 \mathrm{~h}$ after which the cells were harvested for isolation of total RNA and preparation of whole-cell protein lysate. (A) Schematic representation of the splicing reporter, $p \beta$-gal ex12-ex13. The construct contains mouse genomic DNA encompassing exon 12 (nucleotides 37-93), intron 12, and exon 13 of the G6PD gene ligated to $\beta$-galactosidase and under the transcriptional control of a CMV promoter. (B) Representative western blot of whole-cell lysate probed with antibodies against phosphorylated SR proteins (top panels), SRSF3 (middle panels) and lamin A/C (bottom panels). (C) Quantitation of SRSF3 and SRSF4 knockdown for $\mathrm{n}=3$ separate siRNA experiments in HepG2 cells. The "*" indicates a 
significant difference $(p<0.05)$. (D) Total RNA isolated from HepG2 cells following knockdown was used to measure the amounts of RNA for the splicing reporter ( $p \beta$-gal ex12-ex13), the endogenous G6PD transcript, cyclophilin B, and the amount of spliced reporter RNA (spliced RNA). The "*" symbol indicates a significant difference $(p<0.05), n=3$ independent knockdown experiments.

Figure 6. SiRNA-mediated depletion of SRSF3 reduces splicing of G6PD in HeLa cells. HeLa cells were transiently transfected with the splicing reporter (Fig. 5A) and siRNA against SRSF3, SRSF4, SRSF5, and the non-targeting control. (A) Whole cell lysates were prepared from HeLa cells $24 \mathrm{~h}$ after transfection. Western analysis was performed with antibodies against multiple SR proteins (mAB104), SRSF3, and lamin A/C. A representative blot is shown. (B) Quantitation of SR protein knockdown for $n=5$ (SRSF3) and $n=4$ (SRSF4 and SRSF6) separate experiments. (C) The expression of the transiently transfected G6PD reporter ( $p \beta$-gal ex12-ex13), endogenous G6PD gene, and cyclophillin B gene in each of these experiments was measured by real-time RT-PCR. The "*” indicates a significant difference $(p<0.05)$.

Figure 7. SRSF3 specifically binds to the splicing regulatory element in vivo and refeeding enhances the binding of SRSF3 to the splicing regulatory element in mouse liver. (A) HepG2 cells were crosslinked with formaldehyde and SRSF3 was immunoprecipitated from the lysates. G6PD RNA bound to SRSF3 was detected using primers, which amplify the region between nucleotides 11 to 85 in exon 12 (the regulatory element spans nucleotides 43 to 72 ). Immunoprecipitation with IgG was used as control for non-specific binding to antibody. The amount of RNA detected is expressed relative to the input RNA in each sample. The data are 
mean \pm SE of $n=4$ separate immunoprecipitations. (B) HepG2 cells were transiently transfected with FLAG®-tagged SRSF3. The cells were chemically-crosslinked 48h after transfection and SRSF3 was immunoprecipitated with anti- FLAG® antibody. The results of 2 separate experiments are shown. (C) Mice were either starved for $18 \mathrm{~h}$ or starved for $18 \mathrm{~h}$ and refed a high-carbohydrate diet for $12 \mathrm{~h}$. RIP was performed on the chemically-crosslinked livers using an antibody against SRSF3 or IgG. G6PD RNA immunoprecipitated with SRSF3 or IgG was detected with real-time RT-PCR and primers to the region of exon 12 (nucleotides 18 to 89 ) that contains the splicing regulatory element (nucleotides 43 to 72 ). The amount of detected RNA is expressed relative to the input RNA in each sample. The data are the mean \pm SE of $n=3$ mice per group. (D) Total RNA was isolated from a portion of each liver used in the RIP analysis. G6PD mRNA was measured using real time RT-PCR as previously described. Each bar in the mean \pm SE of $n=3$ mice. The "*” indicates a significant difference $(p<0.05)$. 


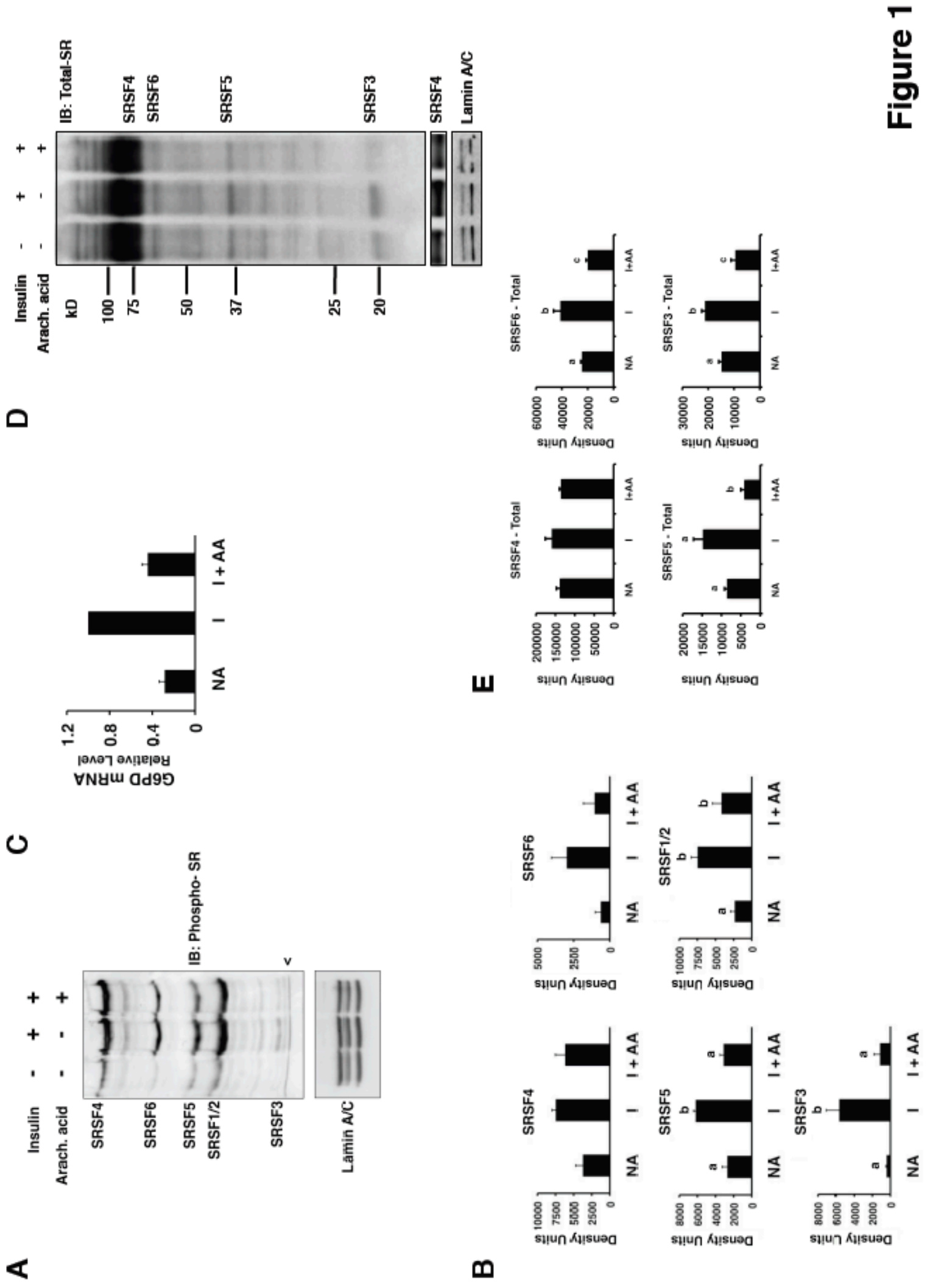



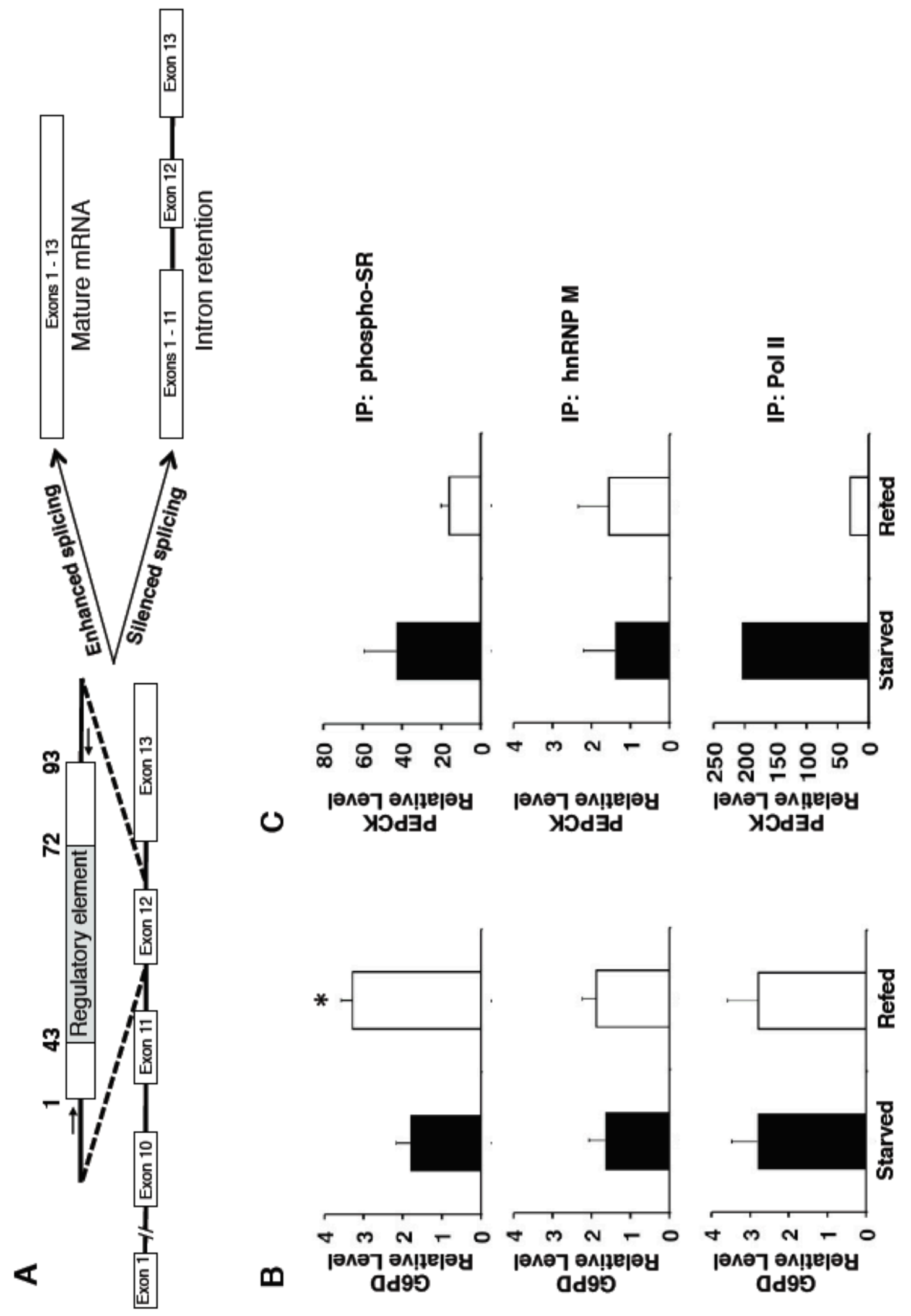


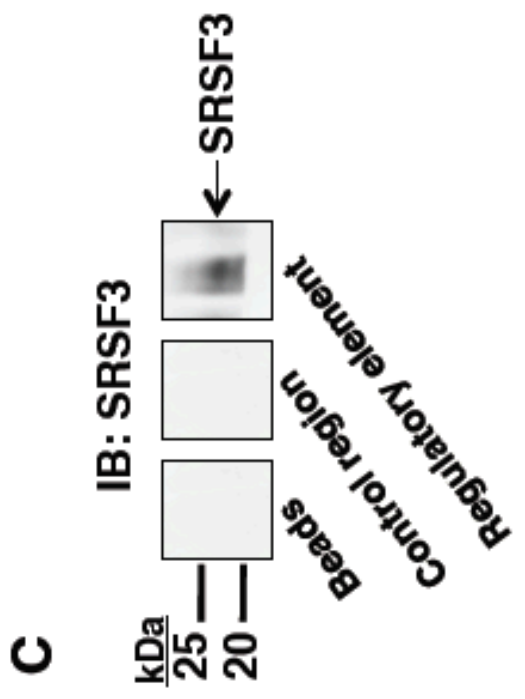

볼

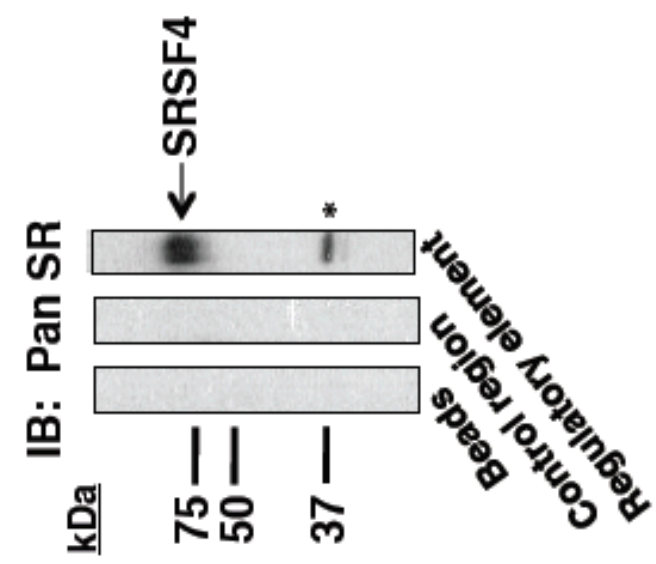

$m$

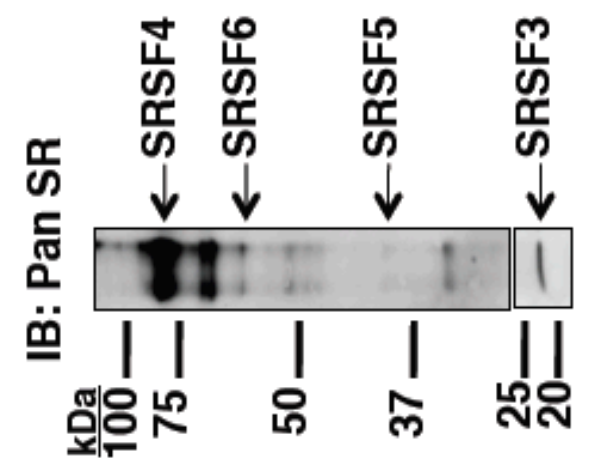

4 

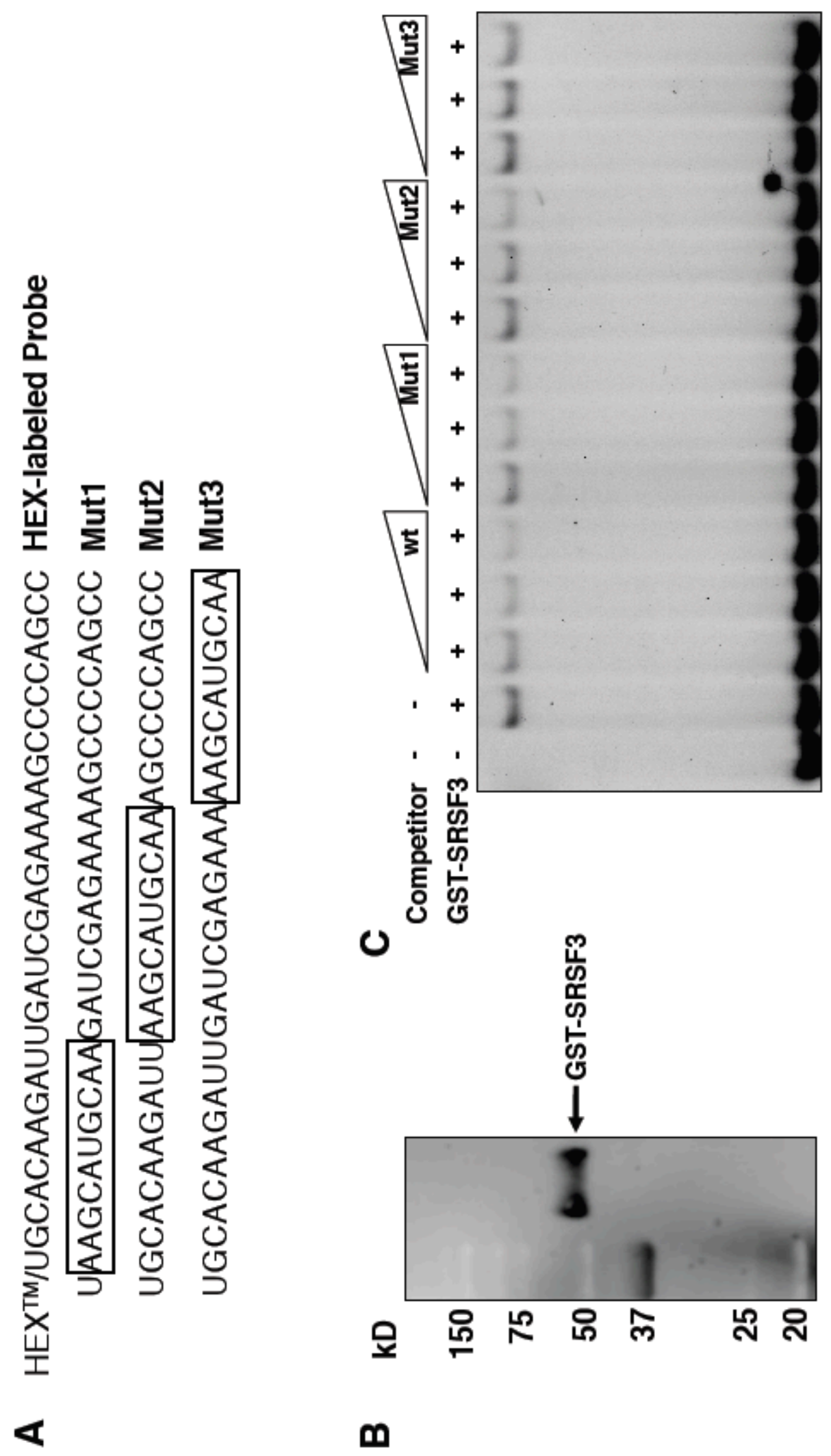

+
일
은 


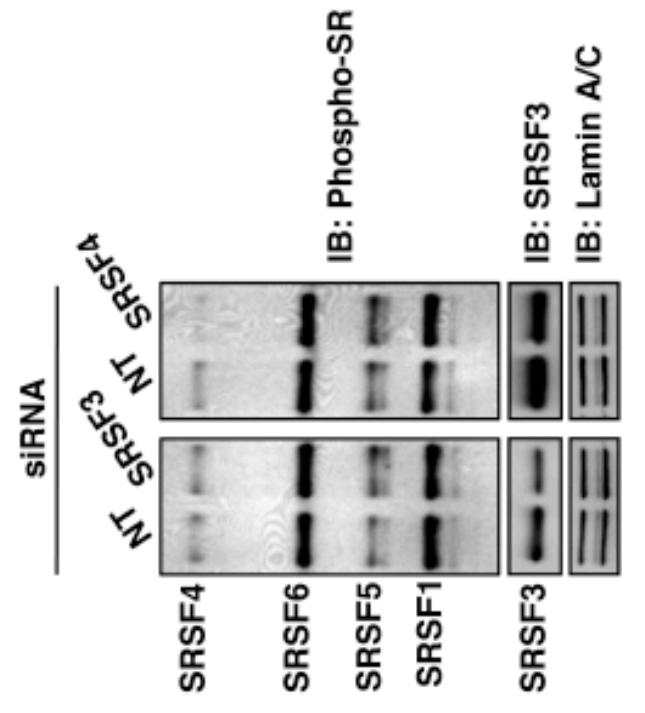

๓

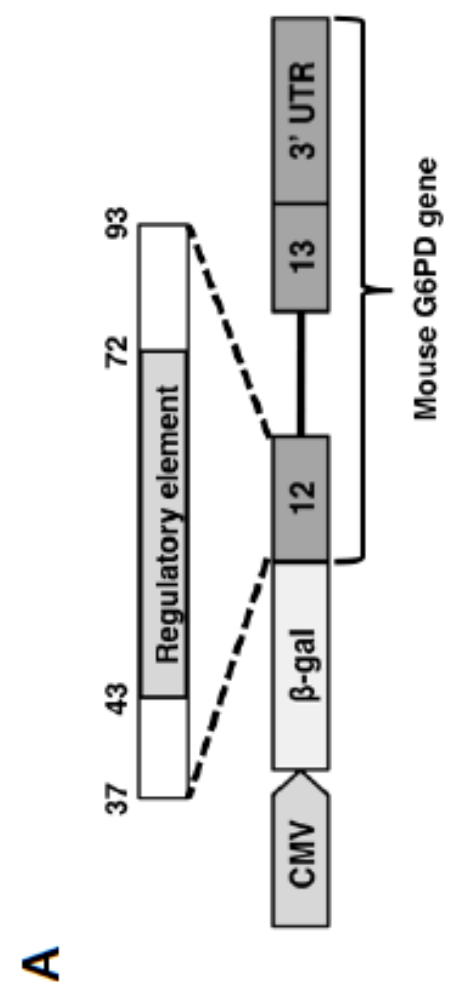

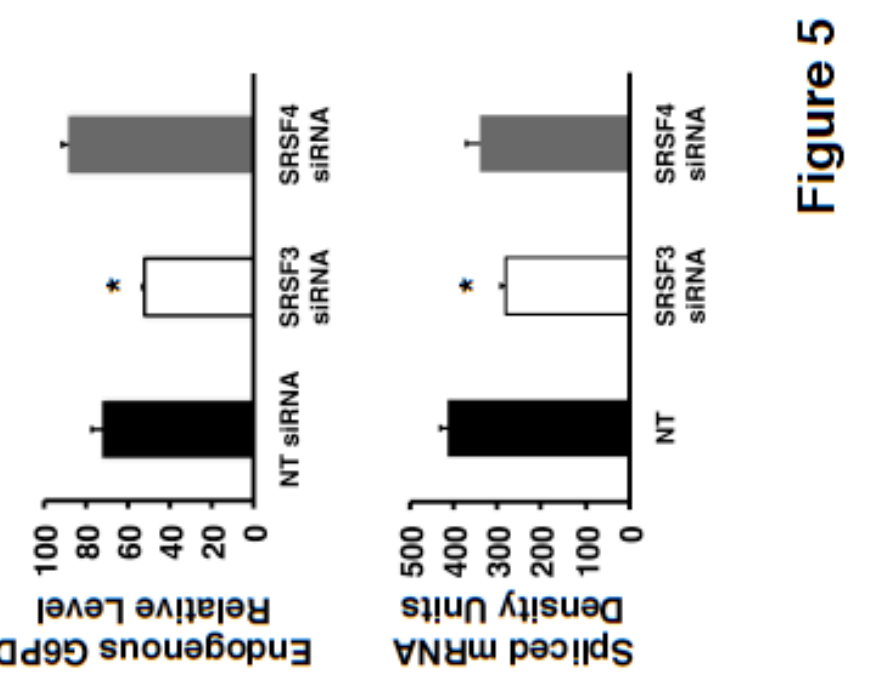

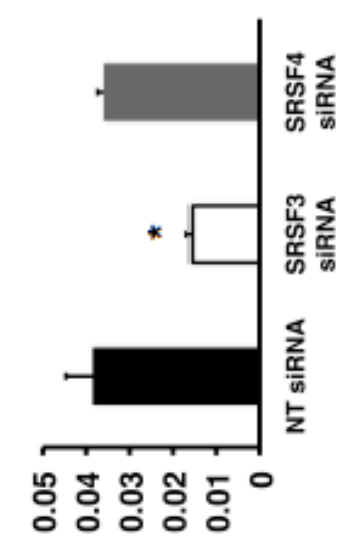

юөләך әк!џејөу

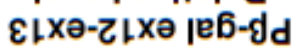

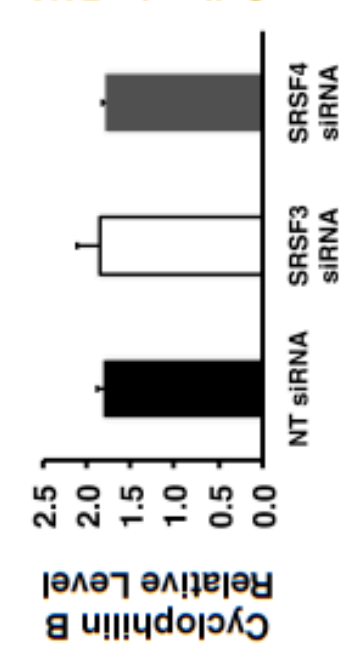




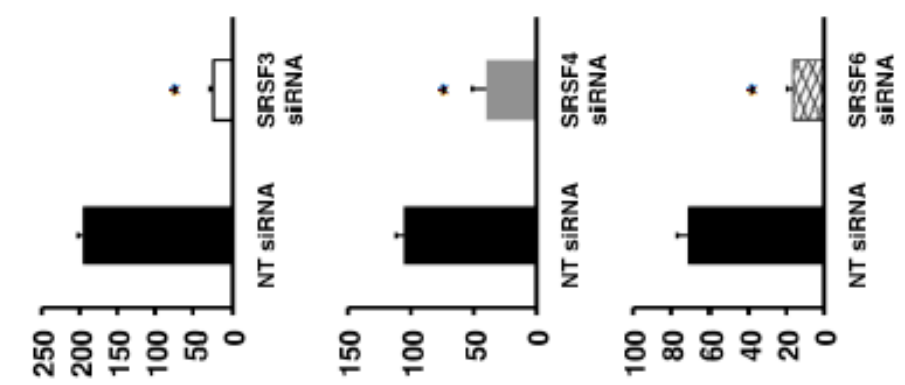

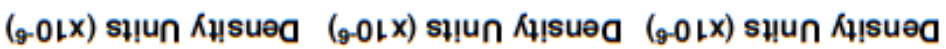
$\overline{\varepsilon\lrcorner S \mathrm{US}}$ tySUS

$\overline{9 J S Y S}$

$\boldsymbol{m}$

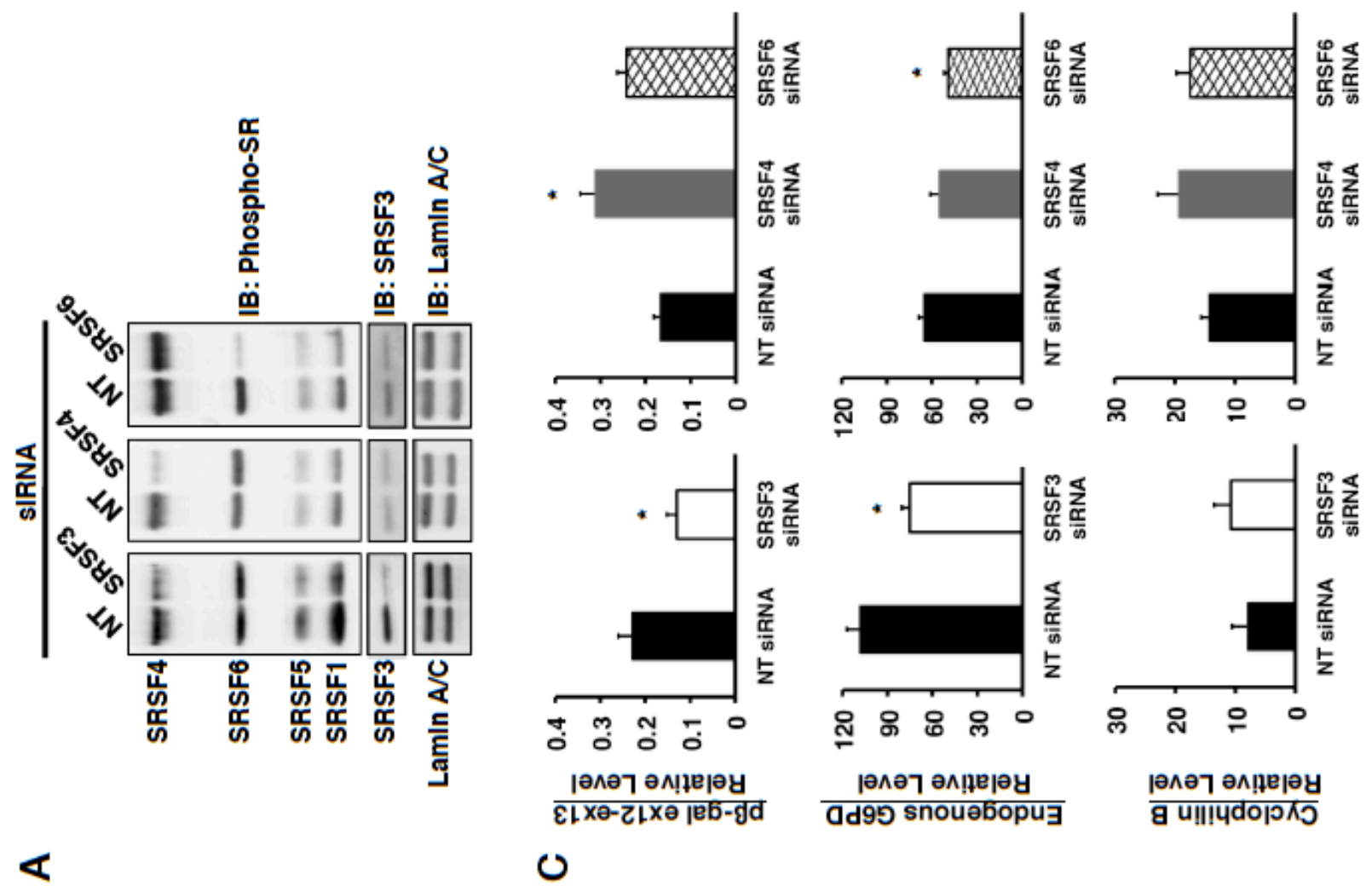




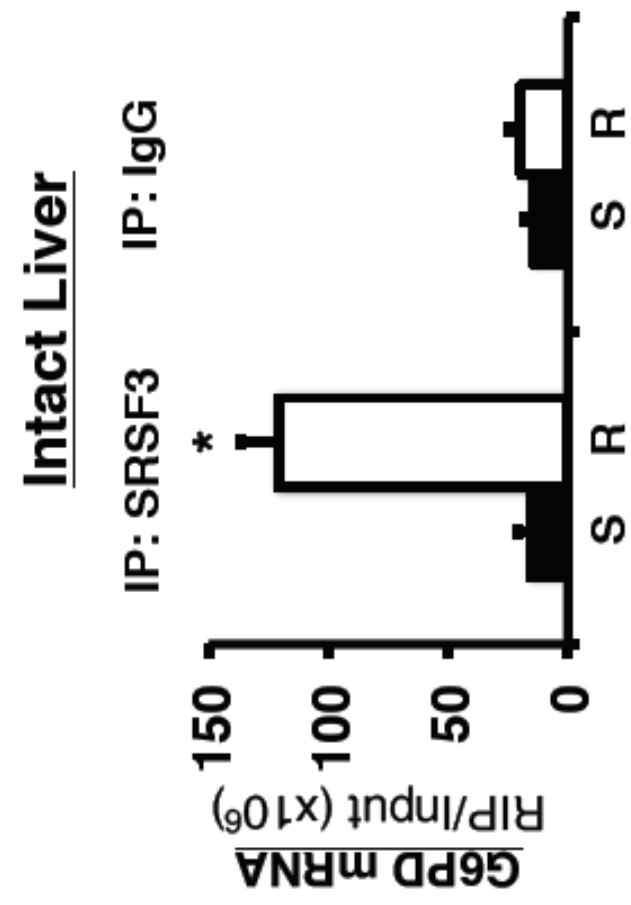

0

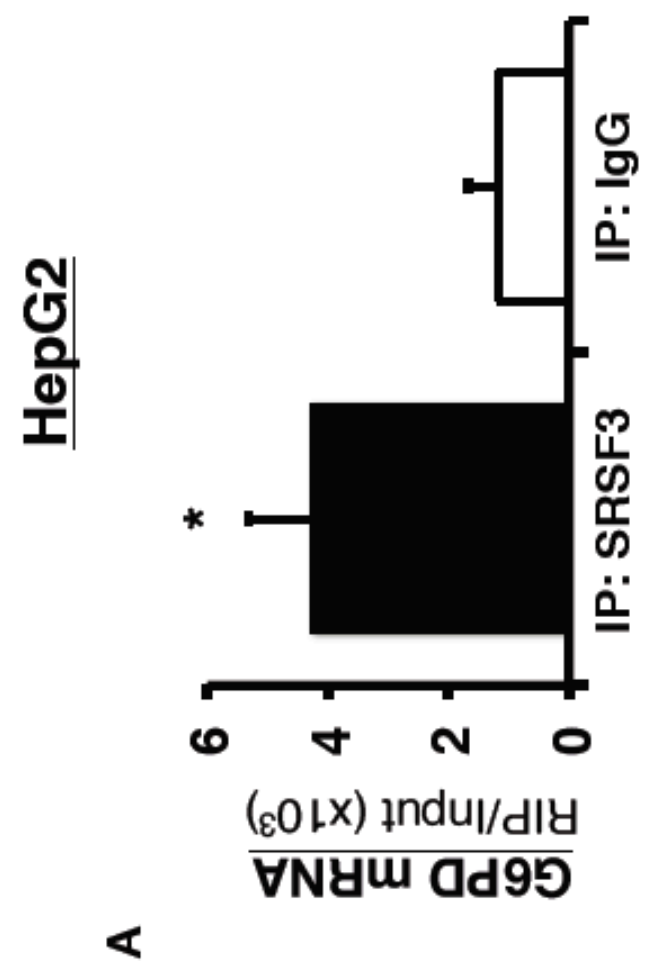

口

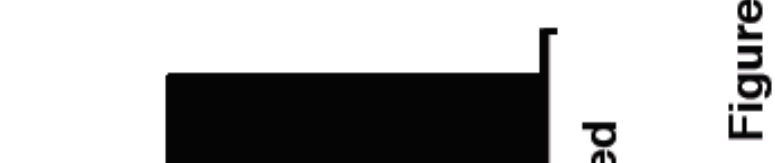

ํํำ
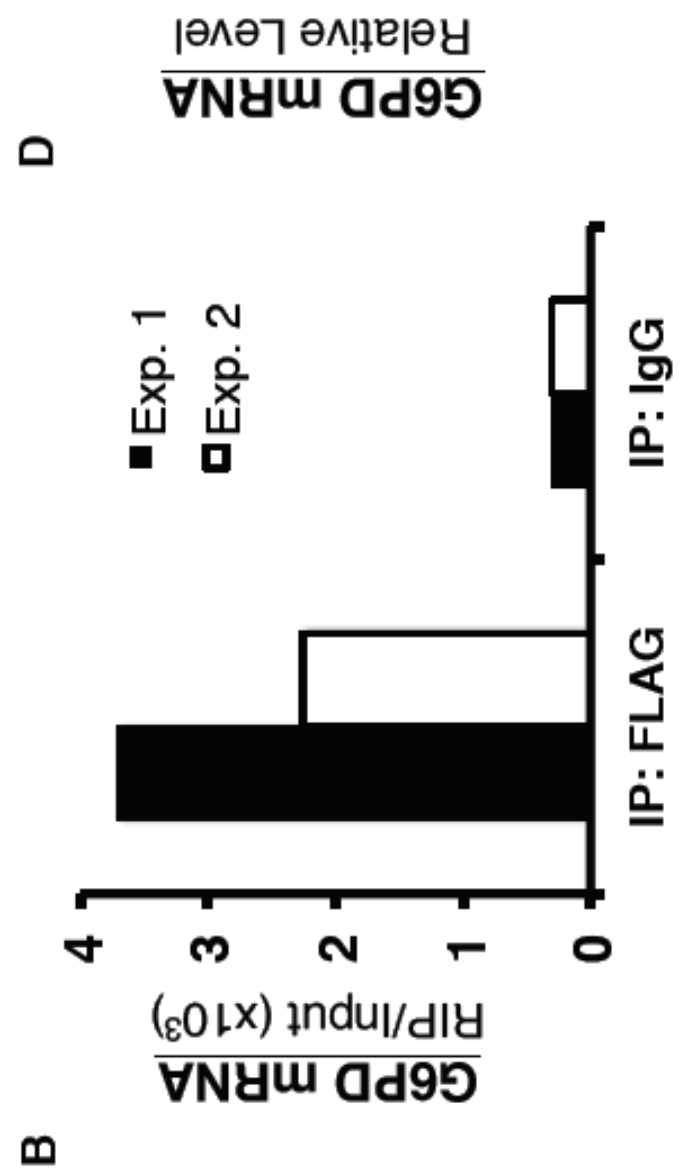


\title{
CHAPTER 3 - PAPER PUBLISHED IN THE JOURNAL PLASMID, 2015
}

\section{REPRINTED WITH PERMISSION FROM THE ELSEVIER PUBLICATION GROUP}

\begin{abstract}
Construction and evaluation of an adenoviral vector for the liver-specific expression of the serine/arginine-rich splicing factor, SRSF3
\end{abstract}

Amanda L. Suchanek ${ }^{\S}$ and Lisa M. Salati ${ }^{\S \#}$

$\S^{\S}$ Department of Biochemistry, West Virginia University School of Medicine, Morgantown, WV 26506

\#To whom correspondence should be addressed:

Department of Biochemistry

WVU Health Sciences Center, PO Box 9142

Morgantown, WV 26506

Phone: (304) 293-7759

Email: Lsalati@hsc.wvu.edu 


\section{Abstract}

Serine/arginine-rich splicing factor-3 (SRSF3), alternatively known as SRp20, is a member of the highly-conserved SR protein family of mRNA splicing factors. SRSF3 generally functions as an enhancer of mRNA splicing by binding to transcripts in a sequence-specific manner to both recruit and stabilize the binding of spliceosomal components to the mRNA. In liver, expression of SRSF3 is relatively low and its activity is increased in response to insulin and feeding a high carbohydrate diet. We sought to over-express SRSF3 in primary rat hepatocytes to identify regulatory targets. A standard adenoviral shuttle vector system containing an epitope-tagged SRSF3 under the transcriptional control of the CMV promoter could not be used to produce infectious adenoviral particles. SRSF3 over-expression in the packaging cell line prevented the production of infectious adenovirus particles by interfering with the viral splicing program. To circumvent this issue, SRSF3 expression from the shuttle vector was blocked by placing its expression under the control of the liver-specific albumin promoter. In this system, the FLAG-SRSF3 transgene is only expressed in the target cells (hepatocytes) but not in the packaging cell line. An additional benefit of the albumin promoter is that expression of the transgene does not require the addition of hormones or antibiotics to drive SRSF3 expression in the hepatocytes. Robust expression of FLAG-SRSF3 protein is detected in both HepG2 cells and primary rat hepatocytes infected with adenovirus prepared from this new shuttle vector. Furthermore, abundances of several known and suspected mRNA targets of SRSF3 action are increased in response to over-expression using this virus. This report details the construction of the albumin promoter-driven adenoviral shuttle vector, termed pmAlbAd5-FLAG.SRSF3, that can be used to generate functional adenovirus to express FLAG-SRSF3 specifically in liver. This vector would be suitable for over-expression 
of other splicing factors that could inhibit virus production. In addition, this vector would allow only liver-specific expression of other cargo genes when used in a whole-animal paradigm.

\section{Keywords}

SRSF3, adenovirus, liver-specific over-expression, liver, mRNA splicing, hepatocytes

\section{Abbreviations}

SRSF3, serine/arginine-rich splicing factor-3; SR protein, serine/arginine-rich protein; RRM, RNA recognition motif; RS domain, arginine-serine repeat domain; G6PD, glucose-6phosphate dehydrogenase; $\mathrm{MOI}$, multiplicity of infection; AAV, adeno-associated virus; SREBP-1, sterol regulatory element-binding protein 1; ME1, malic enzyme 1; NF-kB, nuclear factor $\mathrm{k}$-light chain enhancer of activated B cells; GusB, $\beta$ - glucuronidase. 


\section{Introduction}

Efficient transfection of target cells both in vitro and in vivo is highly desirable to address the function of specific proteins in the most physiological context. In this regard, adenoviral vectors are an extremely valuable tool for the study of gene expression in a wide variety of tissues [1-4]. Tail vein injection of adenoviral constructs can efficiently deliver a gene of interest to the liver, with measureable expression of the transgene observed as early as $24 \mathrm{~h}$ after injection -in stark contrast to the months required to generate transgenic animals. In both liver and hepatocytes in primary culture, the efficiency of target cell infection is greater than $95 \%$ [5]. In addition to their exceptionally high tropism, adenoviral vectors also have a relatively high packaging capacity in comparison to other virus-based gene delivery systems. Thus, adaptation of adenoviral delivery systems to permit delivery of a wide spectrum of transgenes is highly desirable.

Our laboratory has reported that expression and activity of serine/arginine-rich splicing factor-3 (SRSF3), an important pre-mRNA splicing enhancer protein, is regulated by nutrients in liver [6]. In order to better understand and define the role of SRSF3 on splicing patterns and gene expression in the liver in response to nutrients, we sought to express a $\mathrm{N}$-terminal FLAG-tagged SRSF3 protein in primary rat hepatocytes using adenovirus.

The serine/arginine rich (SR) proteins are a family of trans-acting splicing factors that generally act as enhancers of splicing [7]. The canonical SR protein family members include SRSFs 1-12, previously known as SF2/ASF, SC35, SRp20, SRp75, SRp40, SRp55, 9G8, SRp46, SRp30c, SRp38, p54, and SRrp35, respectively [8]. These proteins regulate both constitutive and alternative splicing in both a tissue- and developmentally-specific manner [918]. Extracellular signaling molecules, such as dietary fat, hormones, and growth factors can affect the splicing process by altering the expression, activity and/or intracellular distribution 
of SR proteins [19-24]. With more than $95 \%$ of human transcripts now thought to undergo alternative splicing [25], deciphering the regulatory mechanisms governing SR protein function is critical to our understanding of the role of RNA splicing in regulating physiological processes.

SRSF3 is the smallest of the 12 SR proteins, at 164 amino acids. It contains one Nterminal RNA recognition motif (RRM) and an arginine-serine (RS) dipeptide repeat domain spanning approximately 60 amino acids at the C-terminus. SR proteins are concentrated in nuclear compartments called speckles, but a few-SRSF3 included-shuttle between the nucleus and the cytoplasm to participate in non-splicing functions including mRNA transport and cap-dependent translation [17]. In mouse models, SRSF3 knockout embryos die prior to blastocyst formation, thus, while structurally similar to other SR proteins, SRSF3 is essential and functionally non-redundant [26]. Furthermore, conditional knockout of SRSF3 in mouse liver interferes with hepatocyte development and negatively affects hepatic lipogenic and cholesterol biosynthetic pathways [27]. SRSF3 is also known to play critical roles in the production of viral particles from several families, including picoronaviridae, retroviridae, adenoviridae, papillomaviridae, and herpesviridae [28-33].

The specific cellular functions of SRSF3 are not as extensively characterized as some other SR proteins, with only a handful of regulatory targets having been identified in vitro-its own transcript included [34-37]. In liver, changes in pathway activity due to lack of SRSF3 expression have not identified direct targets of this protein's splicing regulatory activity. Our laboratory recently demonstrated that splicing of the glucose-6-phosphate dehydrogenase (G6PD) mRNA is enhanced by SRSF3 in liver [6]. Because SRSF3 directly binds to G6PD mRNA in a sequence-specific manner, and SRSF3 phosphorylation and binding is increased 
during feeding, we sought to develop a system in which to over-express SRSF3 in primary hepatocytes and identify additional direct targets of its action.

Hepatocytes in primary culture recapitulate liver-specific traits better than cell lines or hepatomas; however, disadvantages to their use include their poor transfection efficiency and relatively short lifespan in culture ( $<5$ days) [38]. Adenoviral infection provides a mechanism to express proteins in $>95 \%$ of hepatocytes in as little as $24 \mathrm{~h}$ after infection, and can also be used in intact animals for liver-specific gene expression. This report details the construction of an adenoviral expression vector that 1) can be used to produce functional SRSF3 adenovirus in HEK-293T cells, and 2) can be used to express SRSF3 in primary rat hepatocytes. Importantly, expression of SRSF3 in this vector is driven by a tissue-specific promoter and standard adenovirus production protocols can be used to generate functional adenovirus stocks. 


\section{Materials \& Methods}

\subsection{Construction of FLAG-SRSF3 shuttle vectors}

Unless otherwise specified, all enzymes were purchased from New England Biolabs (Ipswich, MA).

The SRSF3-FLAG adenoviral shuttle vector was prepared using the RapAd® CMV Adenoviral Expression System (Cell Biolabs, San Diego, CA). We used an N-terminal FLAGtagged SRSF3 cDNA (a kind gift from Dr. Rozanne Sandri-Goldin, University of CaliforniaIrvine, [30]) in a pCS2 vector backbone. To begin to excise the FLAG-SRSF3 cDNA from the pCS2.FLAG-SRSF3 plasmid, the DNA was linearized by partial digestion with $\mathrm{Xhol}$, due to a second Xhol site in the coding region of SRSF3 (Fig. 1a). The pacAd5 CMV K-N pA vector was digested with Xbal. The ends of both Xhol-digested pCS2.FLAG-SRSF3 DNA and Xbaldigested pacAd5 CMV K-N pA DNA were blunted using DNA Polymerase I, Large (Klenow) Fragment. Both DNA fragments were then digested with BamHI to remove the FLAG-SRSF3 insert in the case of the pCS2.FLAG-SRSF3 vector and to provide the complementary sticky end in the pacAd5 CMV K-N pA vector and the products separated on a 1\% agarose gel. The 903bp FLAG-SRSF3 insert and 6.2kb pacAd5 CMV K-N pA vector bands were excised from the gel and the DNA purified. The excised vector DNA was treated with Antarctic Phosphatase. Overnight ligations were done at ambient room temperature using an 8:1 ratio of insert to vector DNA. The identity of the constructed vector was verified by DNA sequencing and analytical restriction digest and is henceforth referred to as pacAd5-CMVFLAG.SRSF3 (Fig. 1a).

A second vector was constructed that contains FLAG-SRSF3 downstream of the liverspecific albumin promoter. The pLIVE vector (Mirrus Bio, Madison, WI) contains a mouse albumin promoter downstream of the mouse a-fetoprotein enhancer element followed by a 
multiple-cloning site (MCS). FLAG-SRSF3 was excised from the pCS2 vector backbone as described above. The resulting FLAG-SRSF3 insert was ligated into pLIVE vector that was also cut with BamHI and Xhol. The resulting vector is referred to as pLIVE.FLAG-SRSF3 (Fig. 1b).

Final construction of the adenoviral vector required the removal of the CMV promoter from the pacAd5 CMV K-N pA shuttle vector by restriction digest with Sbfl and NotI (Fig. 1C). The digested products were separated on a $1 \%$ agarose gel and the $5.6 \mathrm{~kb}$ fragment containing the pacAd5 vector DNA without the CMV promoter was excised and purified (Fig. 1c). DNA containing the containing the mouse albumin promoter, a-fetoprotein enhancer and FLAG-SRSF3 was produced by amplification of the bracketed region of pLIVE.FLAG-SRSF3 (Fig. 1c) using PCR. The Gibson Assembly® kit was used to ligate the 2.3kb PCR product and the pacAd5 shuttle vector cut with Sbfl and Notl (Fig. 1c). The new shuttle vector is henceforth referred to as pmAlbAd5-FLAG.SRSF3. To prepare the empty vector control (henceforth referred to as "control vector"), the PCR primers used to amplify the bracketed region indicated in Fig. 1c were also used to amplify the pLIVE parent plasmid lacking the SRSF3-FLAG cDNA. The 1.4kb PCR product was ligated into the pacAd5 shuttle vector as above. The identities of the pmAlbAd5-FLAG.SRSF3 and control vectors were verified by DNA sequencing and analytical restriction digest.

\subsection{Production of recombinant FLAG-SRSF3 adenovirus in HEK-293T cells}

Production of recombinant adenovirus was an adaptation of the RapAd® CMV Adenoviral Expression System manufacturer's instructions. Approximately $2 \times 10^{6}$ HEK-293T cells were plated in a $60 \mathrm{~mm}$ tissue culture dish in $3 \mathrm{~mL}$ OptiMEM® (Life Sciences, Carlsbad, CA) supplemented with $4 \%$ fetal bovine serum and without antibiotics. Cells were allowed to 
attach to the plate overnight in a $37^{\circ} \mathrm{C}+5 \% \mathrm{CO}_{2}$ incubator. The shuttle vectors (pacAd5CMV-FLAG.SRSF3, empty pacAd5-CMV-pA, pacAd5-CMV.GFP (a shuttle vector containing GFP provided as part of the RapAd® system), pmAlbAd5-FLAG.SRSF3, and empty pmAlbAd5) and the pacAd5 9.2-100 Ad backbone vector (Cell Biolabs) were linearized with Pacl. The DNA was purified by phenol extraction followed by ethanol precipitation and was resuspended in sterile water.

HEK-293T cells were transfected with $5 \mu \mathrm{g}$ of linearized DNA. The appropriate shuttle vector $(4 \mu \mathrm{g})$ and pacAd5 9.2-100 $(1 \mu \mathrm{g})$ were combined in a $400 \mu \mathrm{L}$-volume of serum-free OptiMEM®. FuGENE® 6 Transfection Reagent ( $9 \mu \mathrm{L}$, Promega, Madison, WI) was added to the DNA and mixed. Complex formation was allowed to proceed for 40 min at room temperature. The HEK-293T cell medium was aspirated and replaced with $2.5 \mathrm{~mL}$ fresh OptiMEM® supplemented with $4 \%$ fetal bovine serum, without antibiotics. The DNAFUGENE® 6 complexes were then added drop-wise to each plate of cells and incubated for $24 \mathrm{~h}$ at $37^{\circ} \mathrm{C}+5 \% \mathrm{CO}_{2}$.

The following day, the media/transfection reagent was aspirated from the HEK-293T cell plates and replaced with $3 \mathrm{~mL}$ complete medium (OptiMEM®) supplemented with $4 \%$ fetal bovine serum and antibiotic/antimycotic). One $\mathrm{mL}$ of additional fresh media was added on day 7 and on day 10 post-transfection. Crude viral lysates were harvested on day 12 posttransfection. Following amplification, virus was purified (ViraBind ${ }^{\mathrm{TM}}$ Adenovirus Purification Kit, Cell Biolabs) and titered (QuickTiter ${ }^{\mathrm{TM}}$ Adenovirus Titer ELISA Kit, Cell Biolabs). Adenovirus produced using the pmAlbAd5-FLAG.SRSF3 shuttle vector DNA is referred to as "albumin FLAG-SRSF3 adenovirus," and adenovirus produced using the pmAlbAd5 empty vector DNA is referred to as "control adenovirus." 


\subsection{Adenoviral infection of HepG2 cells}

One day prior to infection, $5 \times 10^{6}$ HepG2, human hepatoma cells (ATCC, Manassas, VA) were seeded in $100 \mathrm{~mm}$ tissue culture dishes in $10 \mathrm{~mL}$ serum-free Minimum Essential Media (MEM, Gibco®). The next day, the medium was aspirated, replaced with fresh MEM containing adenovirus at the titers indicated in the figure legends and incubated at $37^{\circ} \mathrm{C}+5 \%$ $\mathrm{CO}_{2}$. After $2 \mathrm{~h}$, the infection medium was removed and replaced with $10 \mathrm{~mL}$ complete medium (MEM supplemented with 10\% fetal bovine serum and antibiotic/antimycotic). Wholecell extracts and RNA were collected $48 \mathrm{~h}$ later. This procedure is defined as one experiment; replicate experiments represent the same procedure but performed on different days.

\subsection{Adenoviral infection of primary rat hepatocytes}

All animal experiments were conducted in conformity with the Public Health Service policy on Human Care and Use of Laboratory Animals. Additionally, the Institutional Animal Care and Use Committee of the Division of Laboratory Animal Resources at West Virginia University approved all experimental procedures.

Male Sprague-Dawley rats (180-200 g, Harlan Laboratories, Indianapolis, IN) were fed a standard chow diet (Harlan Teklad). Rats were starved for $18 \mathrm{~h}$ prior to hepatocyte isolation. Hepatocyte isolation and incubation in primary culture was as previously described [39].

Two different infection protocols were tested. In the first, following a $2 \mathrm{~h}$ attachment period at $37^{\circ} \mathrm{C}+5 \% \mathrm{CO}_{2}$, the hepatocytes were washed twice with $1 \times$ HiWoBa medium to remove the newborn calf serum used for cell attachment, and then $1.5 \mathrm{~mL}$ serum-free $1 \mathrm{x}$ HiWoBa was added. Purified adenovirus was added dropwise to each plate at the multiplicities of infection (MOIs) indicated in the figure legends and incubated at $37^{\circ} \mathrm{C}+5 \%$ 
$\mathrm{CO}_{2}$. After a $2 \mathrm{~h}$ infection period, the virus-containing media was removed and replaced with a Matrigel overlay $(0.3 \mathrm{mg} / \mathrm{mL})$ in $1 \times$ HiWoBa and incubated overnight. The next morning, the cells were washed twice with $1 \mathrm{x}$ HiWoBa medium, and then incubated in $1 \mathrm{x}$ HiWoBa medium containing $80 \mathrm{nM}$ insulin for $48 \mathrm{~h}$. Medium was replenished at $24 \mathrm{~h}$, and total RNA and wholecell extracts were collected $48 \mathrm{~h}$ after the addition of insulin.

In the second protocol, a $4 \mathrm{~h}$ cell attachment period was used. After the $4 \mathrm{~h}$ attachment, cells were washed twice, purified adenovirus was added dropwise to each plate as described above, and incubated for an additional $6 \mathrm{~h}$. The second protocol matched the first from this point forward. 


\section{Results \& Discussion}

To discover new targets of SRSF3 in liver, we adopted the approach of adenoviral over-expression to produce tagged SRSF3. Using standard techniques, FLAG-SRSF3 was sub-cloned into an adenoviral shuttle vector (pacAd5) and the linearized vector was transfected into HEK-293T cells. Unexpectedly, HEK-293T cells transfected with pacAd5CMV-FLAG.SRSF3 (Fig. 1a) failed to produce virus, while cells transfected with control vector (pacAd5 CMV K-N pA or pacAd5-CMV.GFP) produced virus with titers of $7.0 \times 10^{9}$ and $5.5 \mathrm{x}$ $10^{10} \mathrm{ifu} / \mathrm{mL}$, respectively. Increasing the ratio of shuttle vector DNA to backbone from 3:1 to 2:1 also did not yield measurable viral titers. To determine if the problem was transfection efficiency or viral production, Western analysis was performed on cell lysates using two different ratios of shuttle vector to backbone. In both cases, FLAG-SRSF3 was detected (Fig. 2a, lanes 2 and 3) and its abundance was greater than 1.5-fold over endogenous SRSF3 as measured with the endogenous SRSF3 antibody. This indicated that transfection was occurring and FLAG-SRSF3 was robustly expressed in HEK-293T cells. Thus, the lack of virus production was not due to problems with transfection efficiency.

The expression of adenovirus genes is tightly regulated by changes in pre-mRNA splicing patterns [40]. Distinct mRNA species accumulate at specific times during adenovirus infection depending on viral splice-site selection. Splice-site switching is a temporally regulated process that occurs when the viral splicing program switches from producing transcripts that encode the early viral proteins-those involved in suppression of the host immune response and replication of the viral genome-to transcripts that encode the late viral proteins - proteins involved in packaging of the newly-produced virus [40, 41]. To accomplish this, the viral splicing machinery favors proximal splice sites during early infection, and distal splice sites at later time points. As a result, the viral transcription units produce alternatively 
spliced RNA transcripts that are translated into functionally distinct proteins [42]. Previous studies have shown that splice-site selection is significantly influenced by the balance of active SR proteins present in host cells [41]. Over-expression of the SR protein, SRSF1, in HEK-293T cells impairs virus production by inhibiting the proximal-to-distal splice site switch in the early viral E1A and E1B transcription units, resulting in decreased accumulation of mRNA necessary for late viral protein (L1) expression [41]. These studies, coupled with the over-expression of CMV-driven SRSF3 in HEK-293T cells in our hands (Fig. 2a, lanes 2 and 3), are consistent with the hypothesis that SRSF3 may be inhibiting production of functional adenovirus.

We considered several alternative approaches to circumvent these issues. The first was to use a lentivirus expressing a myc-tagged SRSF3. We were able to produce this lentivirus in HEK-293T cells, but primary rat hepatocytes are exceptionally resistant to lentiviral infection $([38,43]$ and data not shown). Another potential alternative we considered was to use an adeno-associated vector (AAV), but AAV systems also require splice-site switching [44] and thus we would likely encounter the same issues.

Potentially, functional adenovirus would be produced if we blocked SRSF3 overexpression in the HEK-293T packaging cells. To accomplish this, we constructed an adenovirus shuttle vector in which the CMV promoter is replaced with a liver-specific promoter (Fig. 1c). In this vector (pmAlbAd5-FLAG.SRSF3), expression of FLAG-SRSF3 is driven by the mouse albumin promoter downstream of the mouse a-fetoprotein enhancer. HEK-293T cells transfected with this vector do not express FLAG-SRSF3 (Fig. 2b, lane 1). Adenovirus was produced at titers ranging from $1.1 \times 10^{10}-2.9 \times 10^{10} \mathrm{ifu} / \mathrm{mL}$ for both pmAlbAd5-FLAG.SRSF3 (albumin FLAG-SRSF3 adenovirus) and pmAlbAd5 only (control adenovirus). 
To ensure that our newly-produced adenovirus would express FLAG-SRSF3 in liver cells, we first infected a human hepatoma cell line, HepG2. FLAG-SRSF3 was robustly expressed in the HepG2 cells and its expression was increased 3-fold over endogenous SRSF3 (Fig. 3, lane 1, top panel). Cells infected with control adenovirus or uninfected cells expressed only the endogenous SRSF3 (Fig. 3, lanes 2 and 3, top and middle panels).

We then infected primary rat hepatocytes with albumin FLAG-SRSF3 or control adenoviruses at MOIs of 5, 10, and 15. In hepatocytes infected with albumin FLAG-SRSF3 adenovirus, we observed a dose-dependent increase in FLAG-SRSF3 protein (Figs. 4a and b). The increase in expression was only $15 \%$ and $30 \%$ more with MOls of 10 and 15 , respectively, as compared to an MOI of 5 (Fig. 4b, left graph). Expression of FLAG-SRSF3 was not detected in the uninfected cells or in cells infected with the control adenovirus (Fig. 4a, right panel, and Fig. 4b, left graph). Expression of endogenous SRSF3 decreased approximately $20 \%$ in hepatocytes infected with albumin FLAG-SRSF3 adenovirus at an MOI of 5 , but expression decreased with increasing $\mathrm{MOI}$ to an amount similar to cells that did not receive virus or received the control virus (Fig. $4 a$ and $b$, right panel). Total SRSF3 mRNA abundance reflects both the endogenous mRNA and FLAG-SRSF3 mRNA.

SRSF3 expression is known to be tightly controlled by an auto-regulatory mechanism $[37,45]$. Increased SRSF3 protein expression promotes the inclusion of exon 4 in the SRSF3 mRNA transcript. SRSF3 exon 4 is known as a "poison exon" because inclusion of this exon creates an in-frame premature termination codon in the transcript, ultimately decreasing the amount of functional SRSF3 protein in the cell [37]. Since the pmAlbAd5-FLAG.SRSF3 shuttle vector contains SRSF3 cDNA lacking exon 4, it is not affected by this negative feedback mechanism. Expression of the FLAG-SRSF3 protein from the albumin FLAGSRSF3 adenovirus is likely triggering the natural negative feedback loop, thus promoting 
exon 4 inclusion in the endogenous SRSF3 mRNA and causing the decrease in protein abundance observed in Figure 4.

SRSF3 regulates the splicing and expression of several genes involved in intermediary metabolism $[6,14,27]$. We next measured the activity of the FLAG-SRSF3 at enhancing expression of these targets. SRSF3 binds to exon 12 of the G6PD transcript, enhancing splicing of this exon and causing an increase in the abundance of G6PD mRNA [6]. Overexpression of SRSF3 by the albumin FLAG-SRSF3 adenovirus in primary rat hepatocytes increased G6PD mRNA abundance 2.2-fold over both uninfected and control adenovirus infected cells (Fig. 5a).

In mice with a liver-specific SRSF3 knockout, several pathways of intermediary metabolism were found to be disrupted in liver, including both glucose production and glucose deposition, and decreases in lipogenesis and cholesterol biosynthesis [14, 27].

In this regard, SREBP-1 is a well-described transcription factor that enhances hepatic lipid metabolism [46], and we measured total SREBP-1 mRNA to determine if SRSF3 overexpression affected the expression of SREBP-1 mRNA. Interestingly, we found that SREBP-1 mRNA was increased approximately 2.5 -fold in albumin FLAG-SRSF3 adenovirus-infected hepatocytes relative to uninfected and control adenovirus-infected hepatocytes (Fig. 5b). The PCR primers used to measure SREBP-1 mRNA do not distinguish between SREBP-1a and SREBP-1c isoforms, but the SREBP-1a isoform is much less abundant in hepatocytes [46]. In addition, we measured the abundance of Malic Enzyme (ME1) mRNA, an enzyme that represents an important link between glucose and lipid metabolic pathways in liver. We found that the amount of ME1 mRNA was increased 1.6-fold in hepatocytes infected with albumin FLAG-SRSF3 adenovirus as compared to uninfected and control infected cells (Fig. 5c). The effect of SRSF3 over-expression via the albumin FLAG-SRSF3 adenovirus was specific to 
distinct transcripts, as the amounts of Nuclear Factor k-Light Chain Enhancer of Activated B Cells (NF-kB) and $\beta$-glucuronidase (GusB) mRNA did not change (Figs. 5d-e). Taken together, our results are consistent with the decreased lipogenesis in the livers of SRSF3 knockout mice and are supportive of the role of SRSF3 in regulating hepatic lipid metabolism. 


\section{Conclusions}

The primary goal of our laboratory has been to understand the mechanisms by which nutritional cues regulate gene expression in liver at the level of mRNA processingspecifically mRNA splicing. We discovered that the splicing activity of SRSF3 is regulated by nutrients and hormones in liver [6], thus we sought to identify additional targets of SRSF3regulated splicing. Adenovirus is an effective tool to express transgenes in hard-to-transfect cells, such as primary rat hepatocytes. Expression of the splicing factor, SRSF3, challenged the use of this system because the splicing factor abrogated the production of functional viral particles by the packaging cells (HEK-293T). Use of the liver-specific promoter from the albumin gene circumvented this problem because this promoter is inactive in HEK-293T cells (Fig. 2b), but is active in both primary hepatocytes and hepatoma cells (Figs. 3 and 4). The albumin promoter has a further advantage in the target cells in that it does not require the addition of exogenous compounds to drive transgene expression in the liver. Inducible promoter systems often require addition of hormones and/or antibiotics to the culture media that could have undesirable pleiotropic effects in primary hepatocytes, confounding the interpretation of changes in target gene expression. We have confirmed that the FLAGSRSF3 protein expressed by this adenovirus is active in the hepatocytes (Figs. 4 and 5). In this regard, over-expression of SRSF3 increased the abundance of transcripts for 3 key proteins in hepatic lipid metabolism: G6PD, SREBP1, and ME1 mRNA (Figs. 5a-c), while expression of two unrelated transcripts, NF-kB and GusB, remained unchanged (Figs. 5d-e). The adenoviral vector described herein is, to our knowledge, the first to enable overexpression of SRSF3 in primary hepatocytes, and thus represents a novel tool to study the activity of SRSF3 and its critical role in the regulation of hepatic metabolic pathways. 


\section{Acknowledgements}

This work was supported by NIH/NIDDK grant DK46897 (to L. M. S.). The authors wish to thank the current and former members of the F.B. Hillgartner, P. Stoilov, and R. Leonardi laboratories in the West Virginia University Department of Biochemistry for their helpful suggestions, reagents, and criticism. We also thank Dr. Rozanne Sandri-Goldin, University of California-Irvine for the pCS2.FLAG-SRSF3 plasmid and the Genomics Core Facility at West Virginia University for DNA sequencing. 


\section{REFERENCES}

1. Rosenfeld, M.A., et al., Adenovirus-mediated transfer of a recombinant alpha 1antitrypsin gene to the lung epithelium in vivo. Science, 1991. 252(5004): p. 431-4.

2. Alemany, R., K. Suzuki, and D.T. Curiel, Blood clearance rates of adenovirus type 5 in mice. J Gen Virol, 2000. 81(Pt 11): p. 2605-9.

3. Sen, A., et al., Terminally differentiated neonatal rat myocardial cells proliferate and maintain specific differentiated functions following expression of SV40 large T antigen. J Biol Chem, 1988. 263(35): p. 19132-6.

4. Gomez-Foix, A.M., et al., Adenovirus-mediated transfer of the muscle glycogen phosphorylase gene into hepatocytes confers altered regulation of glycogen metabolism. J Biol Chem, 1992. 267(35): p. 25129-34.

5. Schiedner, G., et al., Genomic DNA transfer with a high-capacity adenovirus vector results in improved in vivo gene expression and decreased toxicity. Nat Genet, 1998. 18(2): p. 180-3.

6. Walsh, C.M., et al., Serine arginine splicing factor 3 is involved in enhanced splicing of glucose-6-phosphate dehydrogenase RNA in response to nutrients and hormones in liver. J Biol Chem, 2013. 288(4): p. 2816-28.

7. Long, J.C. and J.F. Caceres, The SR protein family of splicing factors: master regulators of gene expression. Biochem J, 2009. 417(1): p. 15-27.

8. Manley, J.L. and A.R. Krainer, A rational nomenclature for serine/arginine-rich protein splicing factors (SR proteins). Genes Dev. 24(11): p. 1073-4.

9. Das, R., et al., SR proteins function in coupling RNAP II transcription to pre-mRNA splicing. Mol Cell, 2007. 26(6): p. 867-81. 
10. Fu, X.D., Specific commitment of different pre-mRNAs to splicing by single $S R$ proteins. Nature, 1993. 365(6441): p. 82-5.

11. Sanford, J.R., et al., A novel role for shuttling SR proteins in mRNA translation. Genes Dev, 2004. 18(7): p. 755-68.

12. Sanford, J.R., et al., Splicing factor SFRS1 recognizes a functionally diverse landscape of RNA transcripts. Genome Res, 2009. 19(3): p. 381-94.

13. Sen, S., H. Jumaa, and N.J. Webster, Splicing factor SRSF3 is crucial for hepatocyte differentiation and metabolic function. Nat Commun, 2013. 4: p. 1336.

14. Sen, S., I. Talukdar, and N.J. Webster, SRp20 and CUG-BP1 modulate insulin receptor exon 11 alternative splicing. Mol Cell Biol, 2009. 29(3): p. 871-80.

15. Tacke, R. and J.L. Manley, The human splicing factors ASF/SF2 and SC35 possess distinct, functionally significant RNA binding specificities. EMBO J, 1995. 14(14): p. 3540-51.

16. Tacke, R. and J.L. Manley, Determinants of SR protein specificity. Curr Opin Cell Biol, 1999. 11(3): p. 358-62.

17. Twyffels, L., C. Gueydan, and V. Kruys, Shuttling SR proteins: more than splicing factors. FEBS Journal. 278(18): p. 3246-3255.

18. Wang, J. and J.L. Manley, Overexpression of the SR proteins ASF/SF2 and SC35 influences alternative splicing in vivo in diverse ways. RNA, 1995. 1(3): p. 335-46.

19. Busch, A. and K.J. Hertel, Evolution of SR protein and hnRNP splicing regulatory factors. Wiley Interdisciplinary Reviews: RNA, 2011.

20. Caceres, J.F., et al., Role of the modular domains of SR proteins in subnuclear localization and alternative splicing specificity. J Cell Biol, 1997. 138(2): p. 225-38.

21. Graveley, B.R. and K.J. Hertel, SR Proteins, in eLS. 2001, John Wiley \& Sons, Ltd. 
22. Misteli, T., et al., Serine phosphorylation of SR proteins is required for their recruitment to sites of transcription in vivo. J Cell Biol, 1998. 143(2): p. 297-307.

23. Blaustein, M., et al., Concerted regulation of nuclear and cytoplasmic activities of SR proteins by AKT. Nat Struct Mol Biol, 2005. 12(12): p. 1037-44.

24. Stamm, S., Regulation of alternative splicing by reversible protein phosphorylation. J Biol Chem, 2008. 283(3): p. 1223-7.

25. Tejedor, J.R. and J. Valcarcel, Gene regulation: Breaking the second genetic code. Nature, 2010. 465(7294): p. 45-46.

26. Jumaa, H., G. Wei, and P.J. Nielsen, Blastocyst formation is blocked in mouse embryos lacking the splicing factor SRp20. Curr Biol, 1999. 9(16): p. 899-902.

27. Sen, S., H. Jumaa, and N.J.G. Webster, Splicing factor SRSF3 is crucial for hepatocyte differentiation and metabolic function. Nat Commun. 4: p. 1336.

28. Fitzgerald, K.D. and B.L. Semler, Re-localization of cellular protein SRp20 during poliovirus infection: bridging a viral IRES to the host cell translation apparatus. PLoS Pathog. 7(7): p. e1002127.

29. Verma, D., et al., Epstein-Barr Virus SM protein utilizes cellular splicing factor SRp20 to mediate alternative splicing. J Virol. 84(22): p. 11781-9.

30. Sciabica, K.S., Q.J. Dai, and R.M. Sandri-Goldin, ICP27 interacts with SRPK1 to mediate HSV splicing inhibition by altering SR protein phosphorylation. EMBO J, 2003. 22(7): p. 1608-19.

31. Escudero-Paunetto, L., et al., SR proteins SRp20 and $9 G 8$ contribute to efficient export of herpes simplex virus 1 mRNAs. Virology, 2010. 401(2): p. 155-64.

32. Wong, R.W., et al., Digoxin suppresses HIV-1 replication by altering viral RNA processing. PLoS Pathog, 2013. 9(3): p. e1003241. 
33. Jia, R., et al., Control of the papillomavirus early-to-late switch by differentially expressed SRp20. J Virol, 2009. 83(1): p. 167-80.

34. Liang, H., R.S. Tuan, and P.A. Norton, Overexpression of SR proteins and splice variants modulates chondrogenesis. Exp Cell Res, 2007. 313(8): p. 1509-17.

35. Galiana-Arnoux, D., et al., The CD44 alternative v9 exon contains a splicing enhancer responsive to the SR proteins 9G8, ASF/SF2, and SRp20. J Biol Chem, 2003. 278(35): p. 32943-53.

36. Lim, L.P. and P.A. Sharp, Alternative splicing of the fibronectin EllIB exon depends on specific TGCATG repeats. Mol Cell Biol, 1998. 18(7): p. 3900-6.

37. Jumaa, H. and P.J. Nielsen, The splicing factor SRp20 modifies splicing of its own $m R N A$ and ASF/SF2 antagonizes this regulation. EMBO J, 1997. 16(16): p. 5077-85.

38. Gao, S., et al., Ex vivo gene delivery to hepatocytes: techniques, challenges, and underlying mechanisms. Ann Biomed Eng, 2012. 40(9): p. 1851-61.

39. Tao, $\mathrm{H} .$, et al., Inhibition of the splicing of glucose-6-phosphate dehydrogenase precursor mRNA by polyunsaturated fatty acids. J Biol Chem, 2002. 277(34): p. 31270-8.

40. Imperiale, M.J., G. Akusjnarvi, and K.N. Leppard, Post-transcriptional control of adenovirus gene expression. Curr Top Microbiol Immunol, 1995. 199 ( Pt 2): p. 13971.

41. Molin, M. and G. Akusjarvi, Overexpression of essential splicing factor ASF/SF2 blocks the temporal shift in adenovirus pre-mRNA splicing and reduces virus progeny formation. J Virol, 2000. 74(19): p. 9002-9. 
42. Molin, M., et al., Unscheduled expression of capsid protein IIla results in defects in adenovirus major late mRNA and protein expression. Virus Res, 2002. 83(1-2): p. 197206.

43. Seppen, J., et al., Lentiviral vectors for efficient transduction of isolated primary quiescent hepatocytes. J Hepatol, 2002. 36(4): p. 459-65.

44. Mouw, M.B. and D.J. Pintel, Adeno-associated virus RNAs appear in a temporal order and their splicing is stimulated during coinfection with adenovirus. J Virol, 2000. 74(21): p. 9878-88.

45. Jumaa, H. and P.J. Nielsen, Regulation of SRp20 exon 4 splicing. Biochim Biophys Acta, 2000. 1494(1-2): p. 137-43.

46. Horton, J.D., J.L. Goldstein, and M.S. Brown, SREBPs: activators of the complete program of cholesterol and fatty acid synthesis in the liver. J Clin Invest, 2002. 109(9): p. $1125-31$. 


\section{FIGURE LEGENDS}

FIGURE 1: Cloning schemes used to construct adenoviral shuttle vectors
A) Construction of pacAd5-CMV-FLAG.SRSF3, B) Construction of pLIVE-FLAG.SRSF3, and
C) Construction of pmAlbAd5-FLAG.SRSF3. Note: drawings are not to scale.

FIGURE 2: CMV-driven FLAG-SRSF3 is highly expressed in HEK293T packaging cells while albumin-driven FLAG-SRSF3 is not
A) Western blot of HEK-293T transfected with either a 3:1 (lane 2) or 2:1 (lane 3) ratio of pacAd5-CMV-FLAG.SRSF3 shuttle vector DNA to pacAd5 9.2-100 backbone DNA, or transfection reagent alone (lane 4). Whole-cell extracts were collected $48 \mathrm{~h}$ after transfection. Lysate (10 $\mu$ g protein) was loaded onto 12\% SDS-PAGE gels for Western analysis and probed sequentially with anti-FLAG M2 (1:2000), followed by anti-SRSF3 (1:5000) primary antibodies. B) HEK-293T cells were transfected with pmAlbAd5-FLAG.SRSF3 vector DNA (lane 2), pmAlbAd5 control vector DNA (lane 3), or transfection reagent alone (lane 4). Whole-cell extracts were collected $48 \mathrm{~h}$ after transfection. Lysate (10 $\mu \mathrm{g}$ protein) was loaded onto a $12 \%$ SDS-PAGE gel for Western analysis and probed with the same antibodies as in A).

FIGURE 3: FLAG-SRSF3 is expressed in HepG2 cells infected with albumin FLAG-

\section{SRSF3 adenovirus}

Representative Western blots of $n=2$ separate experiments. HepG2 cells were infected with albumin FLAG-SRSF3 adenovirus (lane 1), control (empty vector) adenovirus (lane 2), or no virus (lane 3 ) for $2 \mathrm{~h}$ at a $\mathrm{MOI}$ of 10 . Whole-cell extracts were collected $48 \mathrm{~h}$ after infection. 
Lysate (10 $\mu$ g protein) was loaded onto 12\% SDS-PAGE gels for Western analysis.

Membranes were probed with the following antibodies: top panel: anti-SRSF3 (1:5000), middle panel: anti-FLAG (1:2000), lower panel: anti- $\beta$-tubulin (1:4000).

FIGURE 4: FLAG-SRSF3 is detected in primary rat hepatocytes infected with albumin

\section{FLAG-SRSF3 adenovirus}

Primary hepatocytes were infected with albumin FLAG-SRSF3 adenovirus, control (empty vector) adenovirus, or no virus for $4 \mathrm{~h}$ at MOls of 5,10 , or 15 . Whole-cell extracts and total

RNA were collected $48 \mathrm{~h}$ after infection. $A$ ) Representative Western blots of $\mathrm{n}=3$ separate experiments. Lysate (10 $\mu \mathrm{g}$ protein) was loaded onto 12\% SDS-PAGE gels for Western analysis and probed sequentially with anti-SRSF3 (1:5000) followed by anti-FLAG M2 (1:2000), and anti- $\beta$-tubulin (1:4000) primary antibodies. B) Densitometric quantitation of $n=$ 3 experiments represented in $4 A$. The ${ }^{*}$ asterisk indicates $p<0.05$. Error bars indicate $+/-$ SEM.

\section{FIGURE 5: Overexpression of FLAG-SRSF3 regulates mRNA abundance of mRNA} targets.

Primary rat hepatocytes were infected with albumin FLAG-SRSF3 adenovirus, control (empty vector) adenovirus, or no virus for $4 \mathrm{~h}$ at a MOI of 10 and total RNA was collected $48 \mathrm{~h}$ later. $A-E)$ The mRNA abundance of each transcript was measured using real-time PCR. Fold changes were calculated using the $\Delta \Delta \mathrm{C}_{\mathrm{t}}$ method with cyclophilin as the endogenous control and expressed relative to the No Virus control. The ${ }^{*}$ asterisk indicates $p<0.05$ and error bars represent SEM of $n=3$ separate experiments. 
FIGURE 1
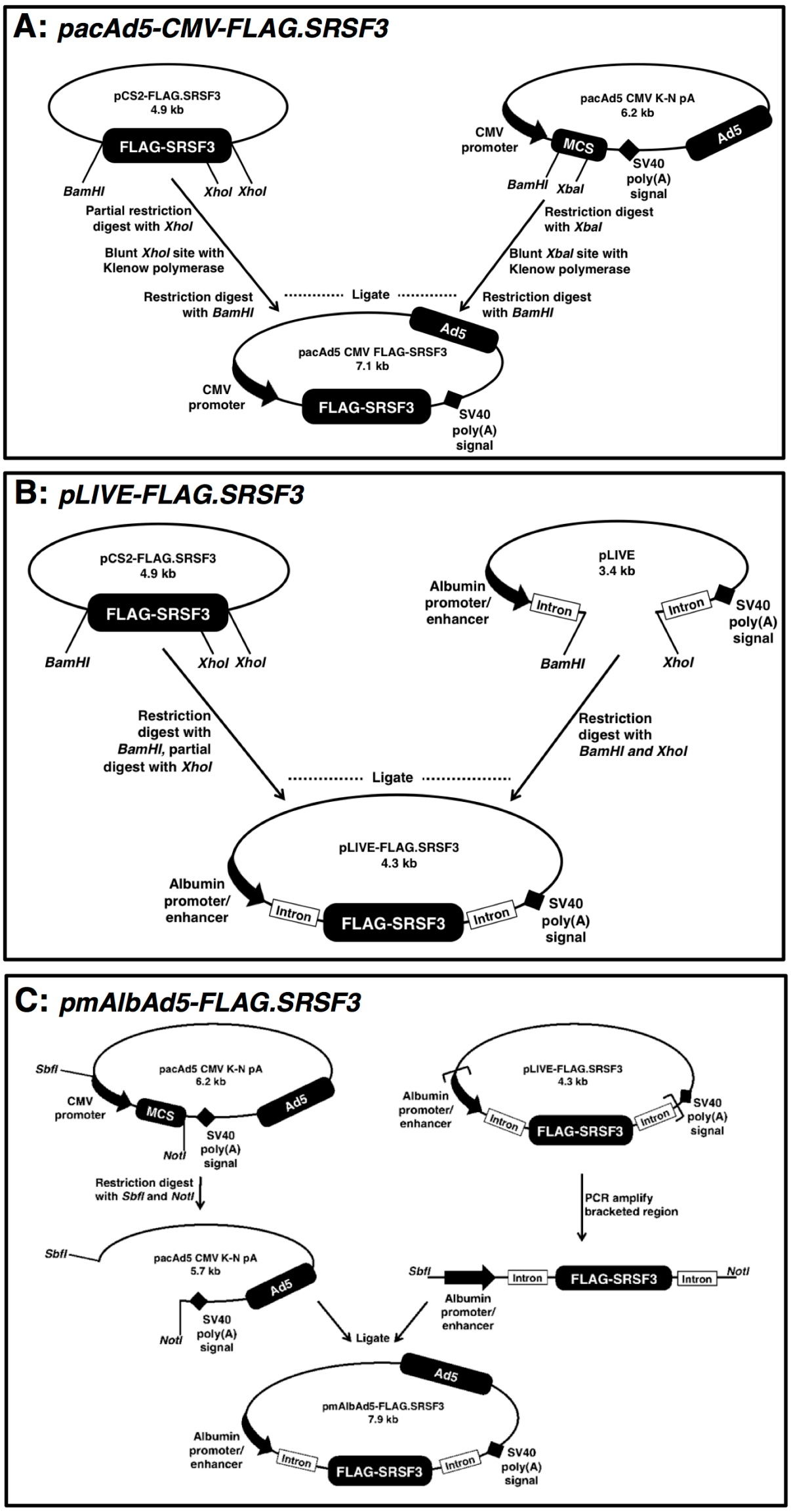


\section{FIGURE 2}
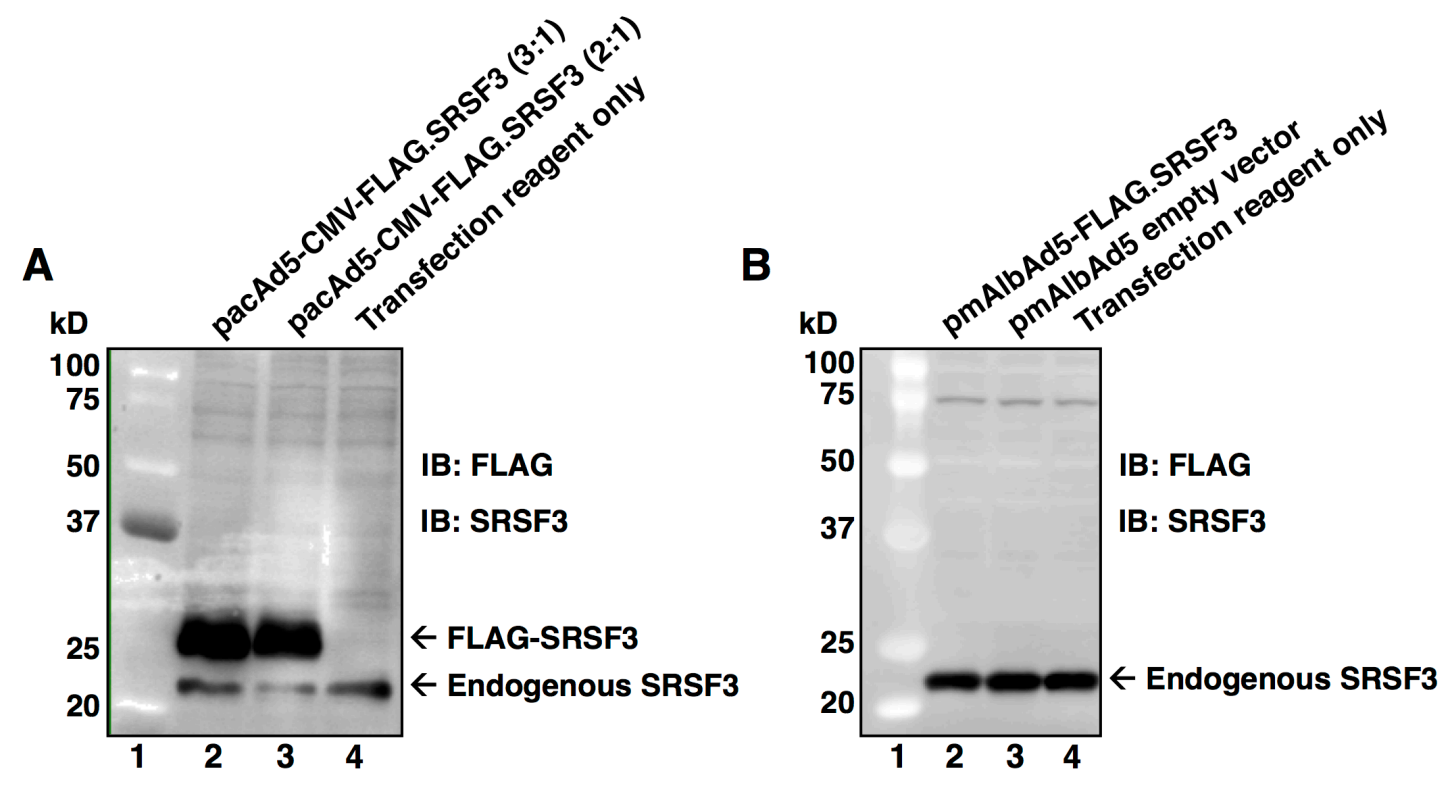

\section{FIGURE 3}

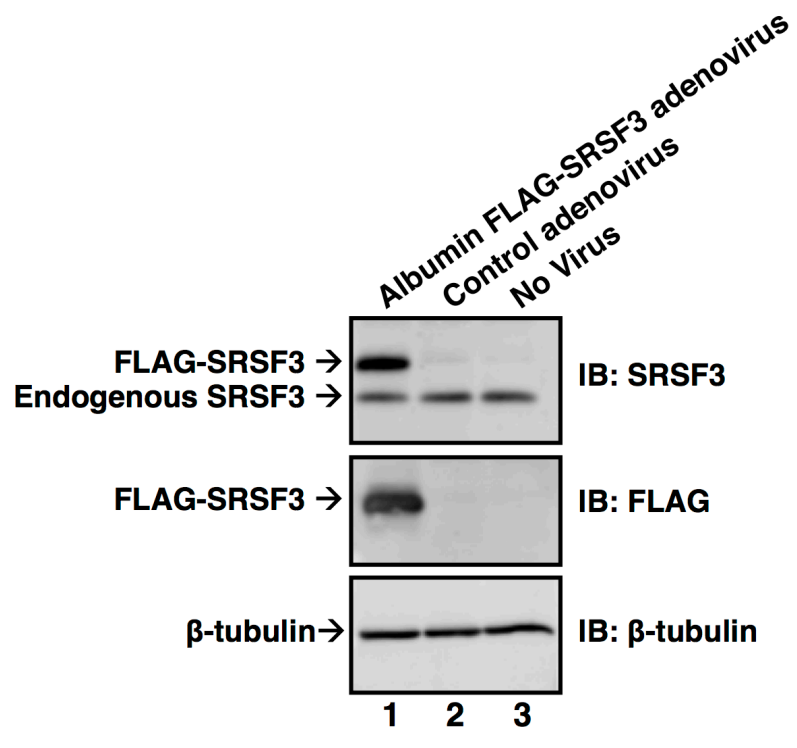


FIGURE 4

A Albumin FLAG-SRSF3 Control
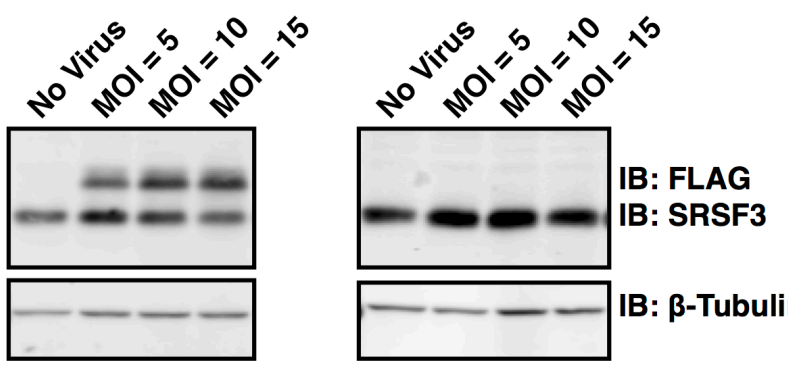

IB: $\beta$-Tubulin

B
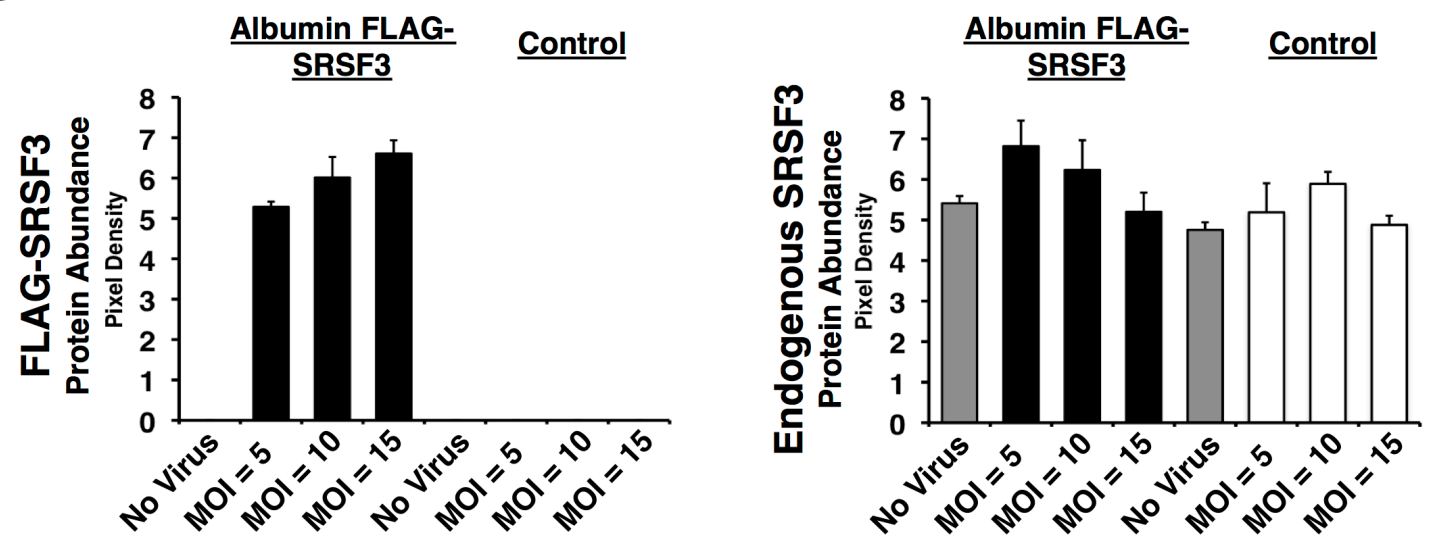
FIGURE 5

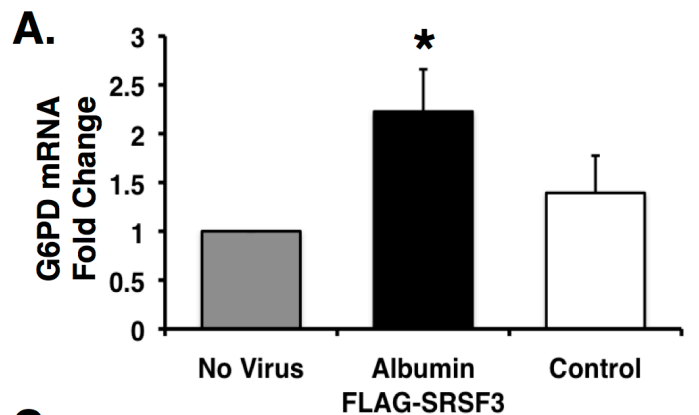

C.
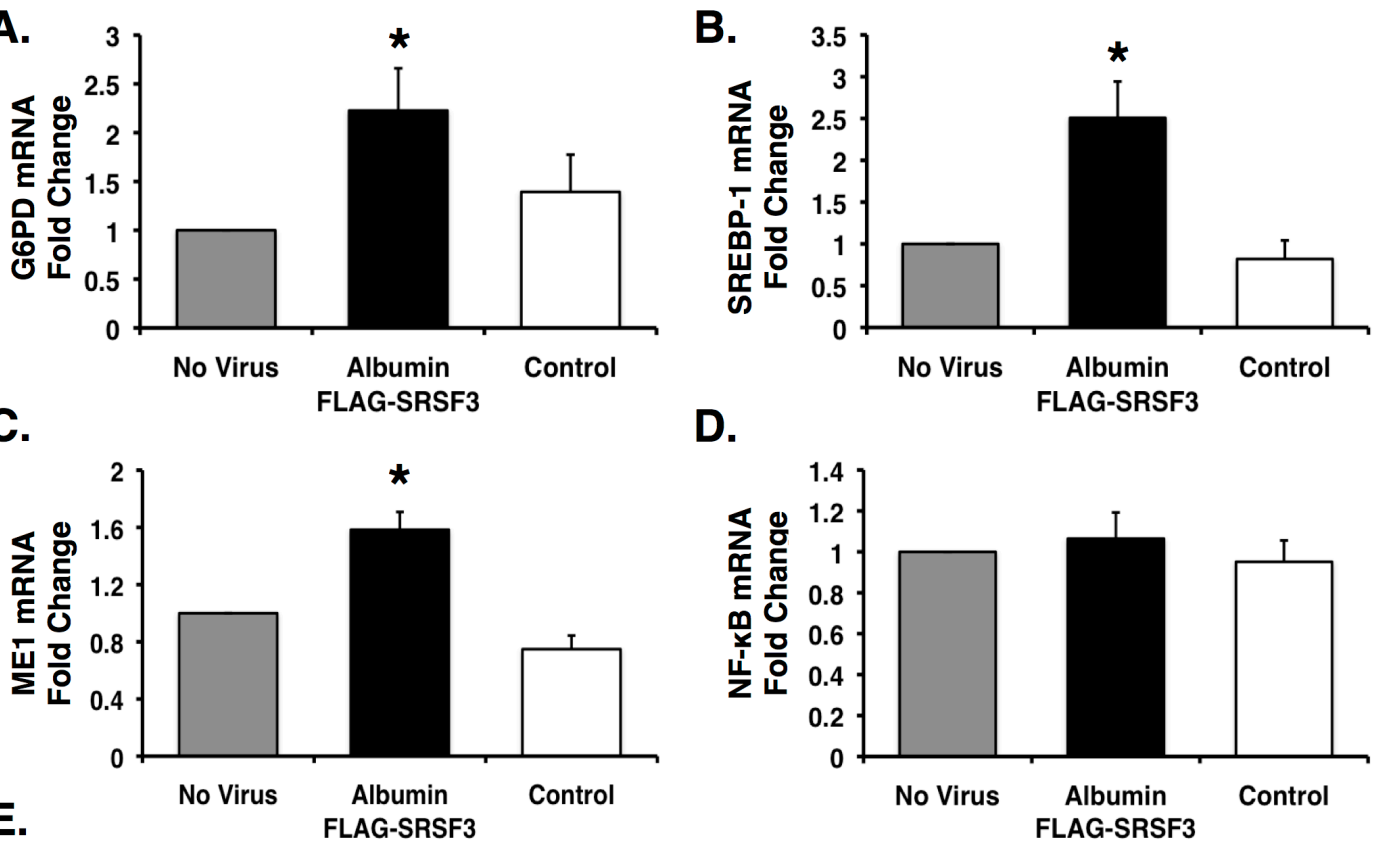

D.

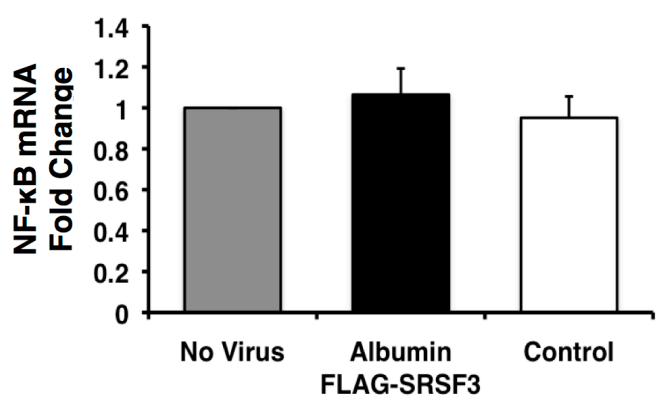




\section{CHAPTER 4:}

\section{INTRON RETENTION IS NOT A MAJOR REGULATOR OF HEPATIC GENE EXPRESSION}

\section{Introduction}

At the cellular level, fat, vitamins, proteins, and other bioactive molecules in the food we eat can alter signal transduction pathways and influence patterns of gene expression. Of the steps in gene expression that can be altered by nutrients, transcription is most often studied. Post-transcriptional steps can be equally important targets for regulation by dietary constituents. Notably, dietary fat has been shown to affect the rate and efficiency of RNA splicing [1, 2], thereby affecting gene expression; however few genes have been identified that undergo this type of regulation [3].

Changes in dietary status can result in major metabolic consequences. In the starved state, pathways that promote utilization of stored energy are activated, including glycogenolysis and lipolysis. These processes allow for the release of glucose and fatty acids from liver and adipose tissue, respectively, in order to provide energy substrates for the body. Following a meal, the release of insulin generally shuts down energy-mobilizing pathways and stimulates glycogen synthesis and lipogenesis to replenish the depleted stores. Dietary composition can exert profound control over the enzymes controlling these metabolic pathways. For example, dietary carbohydrates stimulate the activity of lipogenic enzymes involved in converting the carbon substrates into fatty acids; whereas polyunsaturated fatty acids inhibit lipogenic enzymes [4]. These enzymes include acetyl-CoA carboxylase, fatty acid synthase, malic enzyme, ATP-citrate lyase, and glucose-6-phosphate dehydrogenase (G6PD). Nutritional status affects the rate of transcription of all members of the lipogenic enzyme family-except for G6PD [2, 4]. 
As the rate-limiting enzyme in the pentose-phosphate pathway, G6PD is responsible for the production of reducing equivalents in the form of $\mathrm{NADPH}+\mathrm{H}^{+}$for de novo lipogenesis and protection against oxidative stress [5]. Previous studies in our laboratory have demonstrated that changes in the accumulation of G6PD mRNA in response to dietary status could not be attributed to changes in the rate of transcription or changes in mRNA stability [68]; suggesting that expression of G6PD mRNA is regulated post-transcriptionally at the level of splicing. A splicing regulatory element was later identified within exon 12 of the G6PD mRNA that is responsible for mediating the effects of starvation and refeeding on splicing of the nascent transcript [9]. During starvation, the introns surrounding exon 12 are retained, resulting in decreased expression of the enzyme. This observation led us to question whether nutrient status could function as an additional layer of control over gene expression through its effects on alternative splicing patterns. Regulation at the level of splicing could potentially allow the cell to up- or down-regulate expression of genes more rapidly in response to changes in nutrient availability. Alternative splicing via intron retention is an attractive mechanism for this type of regulation. Unlike alternative exon inclusion that alters protein identity, intron retention will lead to degradation of the transcript and thereby decreased production of the protein encoded by that transcript. Our discovery that splicing of G6PD mRNA - particularly intron retention - is regulated by starvation and refeeding in liver led us to hypothesize that this mechanism of regulation is shared by other genes. To extend this paradigm, we performed RNA sequencing ("RNA-seq") on livers from mice that had been starved or starved and then refed. Starvation and refeeding were chosen because these are two unambiguous dietary conditions in which we have observed the largest changes in abundance of G6PD mRNA $[5,7,10]$. The goal of these experiments is to identify additional 
genes that exhibit changes in intron retention in response to starvation and refeeding in a manner similar to G6PD.

\section{Experimental Procedures}

All animal experiments were conducted in conformity with the Public Health Service policy on Humane Care and Use of Laboratory Animals, additionally; the Institutional Animal Care and Use Committee of the Division of Laboratory Animal Resources at West Virginia University approved all experimental procedures.

Collection of total RNA from whole liver : 8-10 week old male C57BL/6J (The Jackson Laboratory, Bar Harbor, ME; 5 mice per condition) were fed a low fat, high carbohydrate diet (Basal Mix TD.00235, Harlan Laboratories, Madison, WI; supplemented with 1\% safflower oil) for one week. Mice in the starved group were fasted for $18 \mathrm{~h}$ prior to liver collection. Mice in the refed group were fasted for $18 \mathrm{~h}$, then refed the low fat, high carbohydrate diet for $24 \mathrm{~h}$. Immediately prior to collection, mice were anesthetized with $3 \%$ isoflurane in oxygen from a precision vaporizer. The livers were cleared via retrograde perfusion with a calcium-free buffered saline solution (0.14 M NaCl, 6.7 mM KCl, 0.02 M HEPES pH 7.4, $25 \mathrm{mM}$ glucose, $250 \mathrm{mM}$ EGTA). For each mouse, one section of the frontal lobe was immediately collected in TriReagent ${ }^{\circledR}$ (ThermoFisher Scientific, Waltham, MA) and homogenized using a PolyTron mechanical tissue disruptor. Another section was snap-frozen in liquid nitrogen for future analysis.

RNA preparation and assessment: Total RNA was extracted from liver and TriReagent ${ }^{\circledR}$ homogenate according to the manufacturers protocol with slight modifications as indicated in Appendix section II b. RNA was treated with DNAse-I following extraction using the TurboDNAse kit (Ambion) as described in Appendix section II c. The quality of the 
RNA was verified by gel electrophoresis and RNA Integrity values (RINs) were determined by Agilent BioAnalyzer analysis (Figures $1 \mathrm{~b}-\mathrm{c}$ ). RIN values range from 1 (completely degraded) to 10 (intact), and RNA-seq requires that RIN values be $>8$ [11]. The RIN values of our samples ranged from 8.9-9.3, indicating that all of our RNA samples were of superior quality. We submitted 8 of our samples (4 starved, 4 refed) to the Genomics Core Facility at Marshall University for sequencing. We requested the ribosomal RNA depletion method for library preparation and 120 million quality reads per sample (100 nt paired-end reads; multiplex 3 samples per lane).

G6PD, FASN, SCD-1, PEPCK, and RPL32 mRNAs were quantified by real-time RTPCR (iCycler, Bio-Rad) using the QuantiTect SYBR Green kit (Qiagen). The amount of each mRNA was calculated using a relative standard curve.

\section{Results}

\section{Validation of overall gene expression changes by real-time PCR}

To confirm that the dietary conditions used in our experiment resulted in changes in overall gene expression that are historically caused by starvation and refeeding, we used real-time PCR to quantify several transcripts. We measured mRNA abundance of G6PD, fatty acid synthase (FASN), phosphoenolpyruvate carboxykinase (PEPCK), and stearoyle-CoA desaturase 1 (SCD1). We found that during refeeding, G6PD mRNA was increased between 8-9-fold (Figure 2a), FASN was increased approximately 10-fold (Figure 2b), SCD-1 was increased 4-5-fold (Figure 2c), and PEPCK was decreased by approximately 50\% (Figure 2d). Other studies have found that in response to refeeding, abundance of G6PD mRNA was increased by 12-15-fold [7], abundance of FASN and SCD-1 mRNA were increased more than 6-fold and 2-fold, respectively [12], and PEPCK mRNA expression decreased by $70 \%$ or 
more [12]. Thus, our results are within the expected changes in gene expression in response to starvation and refeeding and thus our animals were in the expected metabolic state at the time of liver collection.

\section{Analysis of RNA-seq data}

Computational and statistical analyses of the raw RNA-seq data yielded information on both overall gene expression changes and alternative splicing events that occurred in response to starvation and refeeding. We focused our initial studies on the genes that appeared to retain introns differentially between these two dietary states.

Candidate genes were identified as having 1) a false discovery rate (FDR, the probability that the detected event is a false positive [13]) of less than $10 \%$, and 2) a significant $p$ value $(p<0.05)$. Of the hits meeting these criteria, we kept only the events in which the inclusion level difference (IncLD) was greater than 15\%-meaning that the introns meeting the FDR and $p$ value criteria were only counted as candidates if the difference in intron retention frequency between starvation and refeeding differed by $15 \%$ or more. Due to errors in the UNIX code that was used to generate the initial data output, we did not apply the FDR parameter until subsequent re-analyses when those errors were fixed.

In total, our analysis identified more than 50,000 potentially retained introns. The application of our initial statistical parameters (where FDR was not considered) yielded 205 introns that appeared to be retained differentially in response to starvation or refeeding. Much to our surprise, when we included only the hits with a FDR $<10 \%$, this list dwindled to 20 introns (Table 1). Because our library preparation was not "stranded," the possibility that any of these 20 hits were the result of overlapping transcription off the opposite strand could not be ruled out without further validation. To accomplish this, we designed strand-specific 
primers for reverse transcription, so that only the strand of interest would be converted to cDNA. We then designed PCR primer sets that would allow us to measure both spliced and unspliced products from the correct strand using real-time PCR.

In total, we performed strand-specific RT-PCR on 30 potential hits. Four of these hits remained when the FDR was included as a parameter (genes denoted "§" in Table 1). From these experiments, we were able to determine that most of the hits were false discoveries because the apparent retained introns were in fact transcription off the opposite strand. The remaining targets simply failed to validate. Taken together, our results suggest that the majority of the 50,000 introns identified in our initial analysis appear to be unregulated intron retention events. Thus, our conclusion is that intron retention does not appear to be a widespread mechanism for regulating gene expression in response to starvation and refeeding.

\section{Discussion}

The primary focus of this study was to identify additional hepatic genes that were regulated by intron retention in response to starvation and refeeding because of our previous observation that G6PD mRNA is regulated in this manner. We have concluded that, based on our current data set, intron retention is not a widespread phenomenon in hepatic genes. Yet, the possibility remains that other forms of alternative splicing could be involved in the regulation of hepatic gene expression. To this end, we did obtain data on other alternative splicing events that occur in response to starvation and refeeding, including exon skipping (Table 2), mutually exclusive exons (Table 3), alternate 5'- and 3'-splice site usage (Table 4). We also collected data looking at overall gene expression changes under these conditions 
(Table 5). All of these results were obtained using the same statistical parameters to identify significant events as were used in the intron retention data set.

Upon examination of the additional datasets, we found that most of the genes identified as undergoing the other forms of alternative splicing shown in Tables 2-5 encoded isolated proteins involved in wide variety of cellular functions. In other cases, the predicted changes in splicing between starvation and refeeding were too small to be physiologically relevant. The overall expression data reveals a mostly-expected gene expression profile with respect to the metabolic processes that are active during refeeding, such as glycolysis, lipogenesis and cholesterol biosynthesis. Unexpectedly, we found that several genes associated with inflammatory pathway activation were increased during starvation, suggesting that the starvation conditions may have been too severe. Suspicions with respect to the choice of experimental conditions as will be discussed below suggest that further validation of the these alternative splicing events would not be worthwhile.

While our data set may not have supported the hypothesis that intron retention is a widespread nutrient-regulated mechanism of gene expression control in liver, per se, adjustment of certain elements of our experimental design for future experiments could potentially yield more definitive results. For instance, our animal room is on a standard $12 \mathrm{~h}$ light/dark cycle, with the dark cycle beginning at $6: 00 \mathrm{pm}$. The mice in this experiment were starved for $18 \mathrm{~h}$ beginning at 12:00 am, 6 hours into the dark cycle. After the $18 \mathrm{~h}$ starvation period, mice in the refed group were supplied with low fat, high carbohydrate diet at 6:00 pm, and the livers collected at 6:00 pm the following day ( $24 \mathrm{~h}$ later). Mice are nocturnal and are typically active and feeding during the dark cycle. Since we collected the livers of the refed animals at the beginning of their typical feeding cycle $(6: 00 \mathrm{pm})$, the livers of these mice may have more closely resembled the starved livers because they were not actively feeding in the 
hours leading up to collection. This is further supported by our real-time PCR results in figure 1. In comparison to previous studies by ours and other laboratories, the expression changes we observed in response to refeeding were not quite as robust, suggesting that the mice in our studies may not be in the optimal "refed" state. Adjusting the refeeding period of the mice from $24 \mathrm{~h}$ to $12 \mathrm{~h}$ or $36 \mathrm{~h}$ would put collection of the livers at the end of the active feeding cycle and alleviate this potential issue in future experiments.

It is estimated that between $20-30 \%$ of retained introns contain premature termination codons that could target these transcripts for degradation $[14,15]$. That we collected total cellular mRNA from the livers of the mice in our experiments, together with the potential loss of intron-retaining mRNAs due to degradation, suggests that our samples may not accurately reflect the frequency of intron retention that occurs in response to starvation and refeeding. The implication here being that the intron-retaining mRNAs remaining were so diluted in the total cellular mRNA (nuclear + cytoplasmic pools) that we were unable to adequately detect them. To increase the likelihood of detecting intron retention events, collection of only the chromatin-associated mRNA would be a more appropriate choice for future RNA-seq analyses. Isolation of the chromatin-associated mRNA fraction would allow us to specifically evaluate mRNA in the nucleus that is in the process of being transcribed-when splicing is actively taking place. The mRNA pool of each sample would not include as many of the fullyprocessed nuclear mRNAs or those transcripts that have been exported to the cytosol, thus enriching the sample for nascent transcripts and increasing the probability of detecting intron retention events [16].

Finally, regardless of timing or RNA sample preparation method, all future RNA-seq runs should be stranded. Stranded runs will allow us to determine which strand of the cDNA a particular hit is coming from, therefore allowing us to exclude hits that may in fact be the 
result of transcription of another gene off the opposite strand. Additionally, it would make the data validation process significantly more efficient because the additional cost and time required to perform strand-specific RT-PCR would be eliminated.

In conclusion, adjusting the timing of collection of the refed samples and isolating only the chromatin-associated nuclear mRNA fractions could dramatically improve the quantity of data garnered from future RNA-seq experiments. There are still important questions that warrant further studies with regard to the regulation of alternative splicing in response to nutrient availability. 


\section{REFERENCES}

1. Talukdar, I., W. Szeszel-Fedorowicz, and L.M. Salati, Arachidonic acid inhibits the insulin induction of glucose-6-phosphate dehydrogenase via p38 MAP kinase. J Biol Chem, 2005. 280(49): p. 40660-7.

2. Tao, H., et al., Inhibition of the splicing of glucose-6-phosphate dehydrogenase precursor mRNA by polyunsaturated fatty acids. J Biol Chem, 2002. 277(34): p. 31270-8.

3. Ravi, S., R.J. Schilder, and S.R. Kimball, Role of precursor mRNA splicing in nutrientinduced alterations in gene expression and metabolism. J Nutr, 2015. 145(5): p. 841-6.

4. Salati, L.M., et al., Nutritional regulation of mRNA processing. J Nutr, 2004. 134(9): p. 2437S-2443S.

5. Salati, L.M. and B. Amir-Ahmady, Dietary regulation of expression of glucose-6phosphate dehydrogenase. Annu Rev Nutr, 2001. 21: p. 121-40.

6. Amir-Ahmady, B. and L.M. Salati, Regulation of the processing of glucose-6phosphate dehydrogenase mRNA by nutritional status. J Biol Chem, 2001. 276(13): p. 10514-23.

7. Stabile, L.P., et al., Posttranscriptional regulation of glucose-6-phosphate dehydrogenase by dietary polyunsaturated fat. Arch Biochem Biophys, 1996. 332(2): p. 269-79.

8. Amir-Ahmady, B. and L.M. Salati, Regulation of the Processing of Glucose-6phosphate Dehydrogenase mRNA by Nutritional Status. Journal of Biological Chemistry, 2001. 276(13): p. 10514-10523. 
9. Szeszel-Fedorowicz, W., et al., An exonic splicing silencer is involved in the regulated splicing of glucose 6-phosphate dehydrogenase mRNA. J Biol Chem, 2006. 281(45): p. $34146-58$.

10. Hodge, D.L. and L.M. Salati, Nutritional regulation of the glucose-6-phosphate dehydrogenase gene is mediated by a nuclear posttranscriptional mechanism. Arch Biochem Biophys, 1997. 348(2): p. 303-12.

11. Schroeder, A., et al., The RIN: an RNA integrity number for assigning integrity values to RNA measurements. BMC Mol Biol, 2006. 7: p. 3.

12. Liang, G., et al., Diminished hepatic response to fasting/refeeding and liver $X$ receptor agonists in mice with selective deficiency of sterol regulatory element-binding protein1c. J Biol Chem, 2002. 277(11): p. 9520-8.

13. Reiner, A., D. Yekutieli, and Y. Benjamini, Identifying differentially expressed genes using false discovery rate controlling procedures. Bioinformatics, 2003. 19(3): p. 36875.

14. Lewis, B.P., R.E. Green, and S.E. Brenner, Evidence for the widespread coupling of alternative splicing and nonsense-mediated mRNA decay in humans. Proc Natl Acad Sci U S A, 2003. 100(1): p. 189-92.

15. Braunschweig, U., et al., Widespread intron retention in mammals functionally tunes transcriptomes. Genome Res, 2014. 24(11): p. 1774-86.

16. Pandya-Jones, A. and D.L. Black, Co-transcriptional splicing of constitutive and alternative exons. RNA, 2009. 15(10): p. 1896-908. 


\section{FIGURE 1}

A. Experimental outline for RNA-Seq

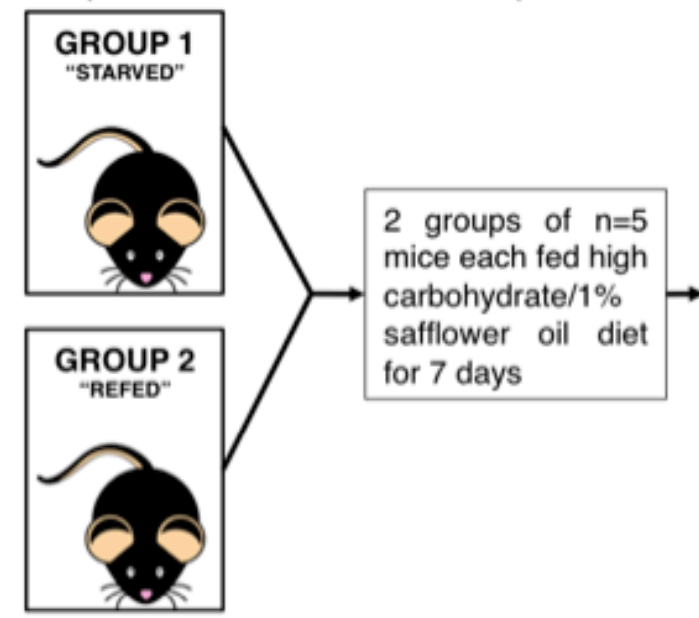

B. TBE/agarose gel to assess quality of total RNA collected from whole mouse liver

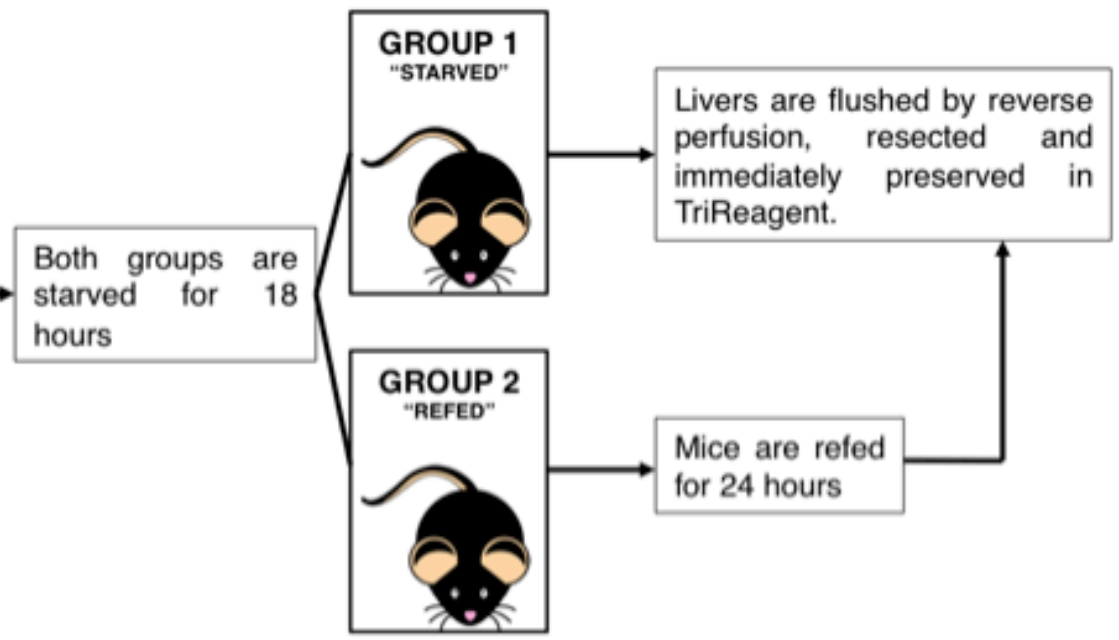

Livers are flushed by reverse perfusion, resected and immediately preserved in Reagent.

C. Representative BioAnalyzer traces of total RNA collected from whole mouse liver.

Starved mice Refed mice 28sm

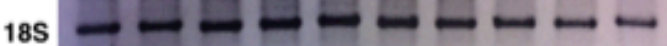
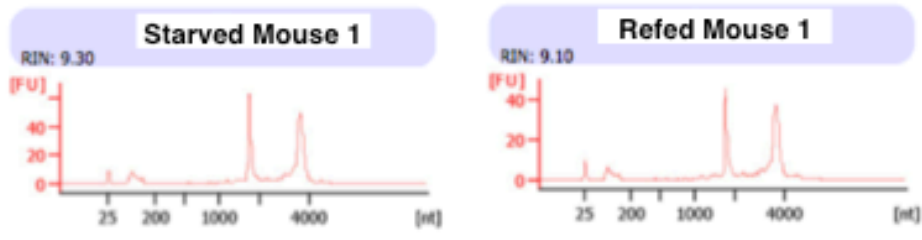

FIGURE 2

a.)

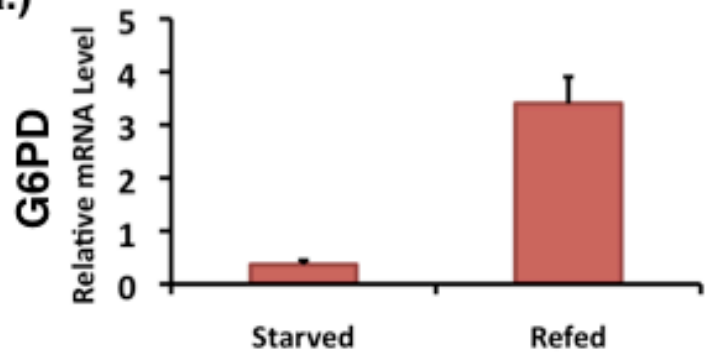

c.)

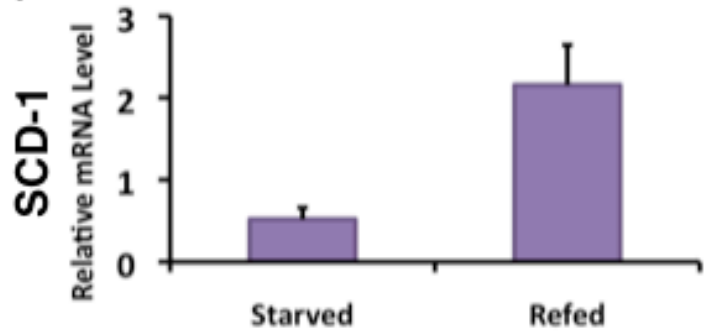

b.)

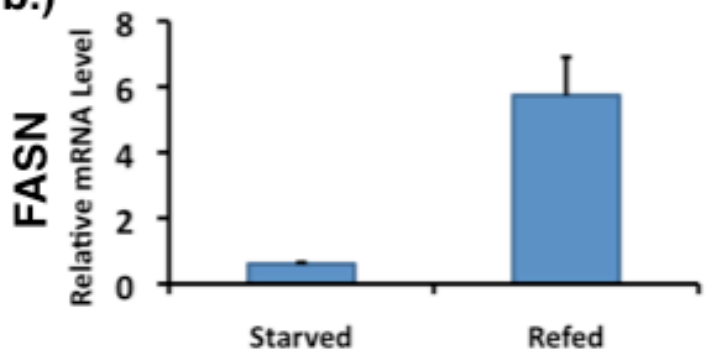

d.)

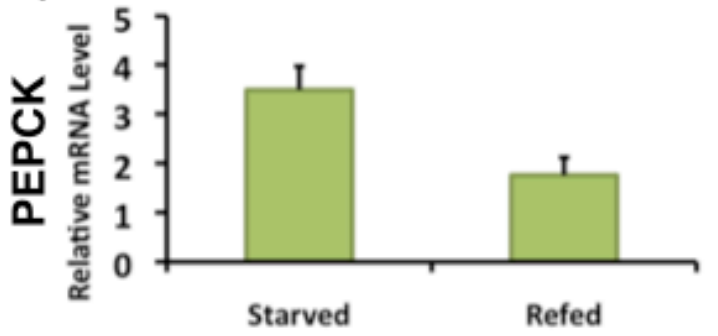

qRT-PCR analysis of genes known to respond to starvation and refeeding. Values were calculated using a relative standard curve and normalized to the endogenous control gene, RPL32. Error bars represent +/- SEM. 


\section{Table 1: Intron Retention Events}

\section{a. Listed by significance}

\begin{tabular}{|c|c|c|c|c|c|c|}
\hline $\begin{array}{c}\text { Gene } \\
\text { Symbol }\end{array}$ & Gene Name & $\begin{array}{c}\text { Genomic Location } \\
\text { of Intron }\end{array}$ & Strand & $p$ value & FDR & Frequency of Retention \\
\hline Ero1lb & ERO1-like beta & chr13:12685786-12687964 & + & $1.11 \mathrm{E}-16$ & $8.02 \mathrm{E}-12$ & $10 \%$ increase during starvation \\
\hline Afmid & arylformamidase & chr11:117693890-117696080 & + & $6.44 \mathrm{E}-15$ & $3.72 \mathrm{E}-10$ & $14 \%$ increase during starvation \\
\hline Gss & glutathione synthetase & chr2:155407769-155413177 & - & $5.89 \mathrm{E}-11$ & $1.89 \mathrm{E}-06$ & $16 \%$ increase during refeeding \\
\hline Uvrag & UV radiation resistance associated gene & chr7:106236685-106247821 & - & 2.42E-09 & 0.0000583 & $12 \%$ increase during refeeding \\
\hline Gas5 & growth arrest specific 5 & chr1:162966375-162966679 & + & $8.64 \mathrm{E}-08$ & 0.0015807 & $14 \%$ increase during refeeding \\
\hline Gas5 & growth arrest specific 5 & chr1:162966983-162967254 & + & 1.86E-07 & 0.002989 & $17 \%$ increase during refeeding \\
\hline Gas5 & growth arrest specific 5 & chr1:162966710-162966950 & + & 5.97E-07 & 0.0075087 & $14 \%$ increase during refeeding \\
\hline Aldh5a1 ${ }^{\S}$ & aldhehyde dehydrogenase family 5 , subfamily $\mathrm{A} 1$ & chr13:25019493-25029119 & - & $6.45 \mathrm{E}-07$ & 0.0077681 & $25 \%$ increase during refeeding \\
\hline Col27a1 & collagen, type XXVII, alpha 1 & chr4:62926713-62933554 & + & $1.61 \mathrm{E}-06$ & 0.0186537 & $15 \%$ increase during refeeding \\
\hline Gss & glutathione synthetase & chr2:155398737-155398830 & - & 1.96E-06 & 0.0217404 & $19 \%$ increase during refeeding \\
\hline Gas5 & growth arrest specific 5 & chr1:162967295-162967418 & + & 2.47E-06 & 0.0264169 & $17 \%$ increase during refeeding \\
\hline$M e 1^{\S}$ & malic enzyme 1, NADP(+)-dependent, cytosolic & chr9:86548325-86571792 & - & $2.79 \mathrm{E}-06$ & 0.0288239 & $20 \%$ increase during starvation \\
\hline Ctdsp2 & CTD small phosphatase 2 & chr10:126429693-126430063 & + & $4.28 \mathrm{E}-06$ & 0.0374014 & $18 \%$ increase during refeeding \\
\hline Dopey $1^{\S}$ & dopey family member 1 & chr9:86442463-86443609 & + & 0.0000044 & 0.0374014 & $21 \%$ increase during refeeding \\
\hline Prodh2 & proline dehydrogenase (oxidase) 2 & chr7:31279543-31291239 & + & 5.83E-06 & 0.0468325 & $15 \%$ increase during refeeding \\
\hline Stab2 & stabilin 2 & chr10:86354395-86356748 & - & 8.73E-06 & 0.0664094 & $13 \%$ increase during starvation \\
\hline Slc29a1 & lute carrier family $29, \mathrm{~m}$ & chr17:45729210-45732430 & - & $9.84 \mathrm{E}-06$ & 0.0711188 & $13 \%$ increase during refeeding \\
\hline Sqle & squalene epoxidase & chr15:59161382-59162279 & + & $9.73 \mathrm{E}-06$ & 0.0711188 & $25 \%$ increase during starvation \\
\hline Gss & glutathione synthetase & chr2:155398911-155403257 & - & 0.0000114 & 0.0753977 & $23 \%$ increase during refeeding \\
\hline Ppp6r2 & protein phosphatase 6 , regulatory subunit 2 & chr15:89105948-89107685 & + & 0.0000108 & 0.0753977 & $18 \%$ increase during refeeding \\
\hline
\end{tabular}

\section{b. Listed alphabetically}

\begin{tabular}{|c|c|c|c|c|c|c|}
\hline $\begin{array}{c}\text { Gene } \\
\text { Symbol }\end{array}$ & Gene Name & $\begin{array}{c}\text { Genomic Location } \\
\text { of Intron }\end{array}$ & Strand & $p$ value & FDR & Frequency of Retention \\
\hline Afmid & arylformamidase & chr11:117693890-117696080 & + & $6.44 \mathrm{E}-15$ & $3.72 \mathrm{E}-10$ & $14 \%$ increase during starvation \\
\hline Aldh5a1 $^{\S}$ & aldhehyde dehydrogenase family 5 , subfamily $\mathrm{A} 1$ & chr13:25019493-25029119 & - & $6.45 \mathrm{E}-07$ & 0.0077681 & $25 \%$ increase during refeeding \\
\hline Col27a1 & collagen, type XXVII, alpha 1 & chr4:62926713-62933554 & + & $1.61 \mathrm{E}-06$ & 0.0186537 & $15 \%$ increase during refeeding \\
\hline Ctdsp2 & CTD small phosphatase 2 & chr10:126429693-126430063 & + & $4.28 \mathrm{E}-06$ & 0.0374014 & $18 \%$ increase during refeeding \\
\hline Dopey $1^{\S}$ & dopey family member 1 & chr9:86442463-86443609 & + & 0.0000044 & 0.0374014 & $21 \%$ increase during refeeding \\
\hline Ero1lb & ERO1-like beta & chr13:12685786-12687964 & + & $1.11 \mathrm{E}-16$ & $8.02 E-12$ & $10 \%$ increase during starvation \\
\hline Gas5 & growth arrest specific 5 & chr1:162966375-162966679 & + & 8.64E-08 & 0.0015807 & $14 \%$ increase during refeeding \\
\hline Gas5 & growth arrest specific 5 & chr1:162966983-162967254 & + & $1.86 \mathrm{E}-07$ & 0.002989 & $17 \%$ increase during refeeding \\
\hline Gas5 & growth arrest specific 5 & chr1:162966710-162966950 & + & 5.97E-07 & 0.0075087 & $14 \%$ increase during refeeding \\
\hline Gas5 & growth arrest specific 5 & chr1:162967295-162967418 & + & 2.47E-06 & 0.0264169 & $17 \%$ increase during refeeding \\
\hline Gss & glutathione synthetase & chr2:155407769-155413177 & - & $5.89 \mathrm{E}-11$ & $1.89 \mathrm{E}-06$ & $16 \%$ increase during refeeding \\
\hline Gss & glutathione synthetase & chr2:155398737-155398830 & - & $1.96 \mathrm{E}-06$ & 0.0217404 & $19 \%$ increase during refeeding \\
\hline Gss & glutathione synthetase & chr2:155398911-155403257 & - & 0.0000114 & 0.0753977 & $23 \%$ increase during refeeding \\
\hline $\mathrm{Me1}^{\S}$ & malic enzyme 1, NADP(+)-dependent, cytosolic & chr9:86548325-86571792 & - & $2.79 \mathrm{E}-06$ & 0.0288239 & $20 \%$ increase during starvation \\
\hline Ppp6r2 & protein phosphatase 6 , regulatory subunit 2 & chr15:89105948-89107685 & + & 0.0000108 & 0.0753977 & $18 \%$ increase during refeeding \\
\hline Prodh2 & proline dehydrogenase (oxidase) 2 & chr7:31279543-31291239 & + & $5.83 \mathrm{E}-06$ & 0.0468325 & $15 \%$ increase during refeeding \\
\hline Slc29a1 & solute carrier family 29, member 1 & chr17:45729210-45732430 & - & 9.84E-06 & 0.0711188 & $13 \%$ increase during refeeding \\
\hline Sqle $^{\S}$ & squalene epoxidase & chr15:59161382-59162279 & + & 9.73E-06 & 0.0711188 & $25 \%$ increase during starvation \\
\hline Stab2 & stabilin 2 & chr10:86354395-86356748 & - & 8.73E-06 & 0.0664094 & $13 \%$ increase during starvation \\
\hline Uvrag & UV radiation resistance associated gene & chr7:106236685-106247821 & - & 2.42E-09 & 0.0000583 & $12 \%$ increase during refeeding \\
\hline
\end{tabular}




\section{Table 2: Exon Skipping Events}

\section{a. Listed by significance}

\begin{tabular}{|c|c|c|c|c|c|}
\hline Gene Name & $\begin{array}{c}\text { Genomic Location } \\
\text { of Exon }\end{array}$ & Strand & $p$ value & FDR & Frequency of Inclusion \\
\hline Itih4 & chr14:31709956-31711042 & + & $3.3 E-12$ & 1.98E-08 & $11 \%$ increase during starvation \\
\hline Cyp3a25 & chr5:146804612-146806440 & - & $5.35 \mathrm{E}-10$ & 0.00000229 & $13 \%$ increase during refeeding \\
\hline Acaca & chr11:83995776-84008939 & + & $8.86 \mathrm{E}-10$ & 0.00000295 & $19 \%$ increase during starvation \\
\hline Huwe1 & chrX:148292456-148294709 & + & 1.09E-07 & 0.00018238 & $12 \%$ increase during refeeding \\
\hline Tm7sf2 & chr19:6067022-6067668 & - & 3.02E-07 & 0.000431301 & $10 \%$ increase during starvation \\
\hline D19Wsu162e & chr19:46673783-46718842 & + & 0.00000076 & 0.00073683 & $16 \%$ increase during refeeding \\
\hline Lpin1 & chr12:16575325-16580465 & - & 0.00000125 & 0.001134135 & $19 \%$ increase during starvation \\
\hline Pex5 & chr6:124353925-124354647 & - & 0.00000163 & 0.001393399 & $11 \%$ increase during refeeding \\
\hline Hnrnpc & chr14:52704066-52723563 & - & 0.00000238 & 0.001782823 & $20 \%$ increase during starvation \\
\hline Itch & chr2:154964008-154989128 & + & 0.00000278 & 0.001948704 & $14 \%$ increase during refeeding \\
\hline Prpf40a & chr2:53016951-53018591 & - & 0.00000323 & 0.002155621 & $11 \%$ increase during starvation \\
\hline Rtn3 & chr19:7509628-7557428 & - & 0.00000417 & 0.002608786 & $13 \%$ increase during starvation \\
\hline Mbnl1 & chr3:60425380-60429045 & + & 0.00000455 & 0.002788029 & $15 \%$ increase during starvation \\
\hline Tmem56 & chr3:120938682-120979905 & - & 0.0000123 & 0.006576559 & $33 \%$ increase during refeeding \\
\hline Apol7a & chr15:77226747-77229367 & - & 0.0000188 & 0.009075003 & $12 \%$ increase during starvation \\
\hline Tra2a & chr6:49202514-49213836 & - & 0.0000245 & 0.011469045 & $27 \%$ increase during starvation \\
\hline Ehhadh & chr16:21775584-21787779 & - & 0.0000723 & 0.026440466 & $11 \%$ increase during starvation \\
\hline Mbnl1 & chr3:60417442-60419541 & + & 0.0000731 & 0.026440466 & $11 \%$ increase during starvation \\
\hline Ctnnd1 & chr2:84462239-84490562 & - & 0.0000907 & 0.03200511 & $14 \%$ increase during starvation \\
\hline Sec22c & chr9:121604860-121614041 & - & 0.00011677 & 0.038083625 & $40 \%$ increase during starvation \\
\hline Dock6 & chr9:21629974-21634657 & - & 0.00012428 & 0.039252765 & $47 \%$ increase during starvation \\
\hline Rbm7 & chr9:48302286-48303259 & - & 0.0001594 & 0.046432076 & $11 \%$ increase during starvation \\
\hline Oma1 & chr4:102998005-103003909 & + & 0.00018758 & 0.054116009 & $12 \%$ increase during refeeding \\
\hline Mbnl1 & chr3:60419695-60426067 & + & 0.00027537 & 0.07122671 & $12 \%$ increase during starvation \\
\hline MII3 & chr5:24830039-24835058 & - & 0.00029526 & 0.073824331 & $18 \%$ increase during starvation \\
\hline Snrnp70 & chr7:52636188-52639173 & - & 0.00029456 & 0.073824331 & $13 \%$ increase during starvation \\
\hline Cdc14b & chr13:64297983-64306578 & - & 0.00035092 & 0.082258325 & $28 \%$ increase during refeeding \\
\hline 2510006D16Rik & chr4:129278379-129279113 & + & 0.00037619 & 0.086162457 & $15 \%$ increase during starvation \\
\hline Eif4h & chr5:135097956-135101235 & - & 0.00040116 & 0.091185016 & $12 \%$ increase during starvation \\
\hline
\end{tabular}




\section{Table 2: Exon Skipping Events}

\section{b. Listed alphabetically}

\begin{tabular}{|c|c|c|c|c|c|}
\hline Gene Name & $\begin{array}{c}\text { Genomic Location } \\
\text { of Exon }\end{array}$ & Strand & $p$ value & FDR & Frequency of Inclusion \\
\hline 2510006D16Rik & chr4:129278379-129279113 & + & 0.00037619 & 0.086162457 & $15 \%$ increase during starvation \\
\hline Acaca & chr11:83995776-84008939 & + & $8.86 \mathrm{E}-10$ & 0.00000295 & $19 \%$ increase during starvation \\
\hline Apol7a & chr15:77226747-77229367 & - & 0.0000188 & 0.009075003 & $12 \%$ increase during starvation \\
\hline Cdc14b & chr13:64297983-64306578 & - & 0.00035092 & 0.082258325 & $28 \%$ increase during refeeding \\
\hline Ctnnd1 & chr2:84462239-84490562 & - & 0.0000907 & 0.03200511 & $14 \%$ increase during starvation \\
\hline Cyp3a25 & chr5:146804612-146806440 & - & $5.35 \mathrm{E}-10$ & 0.00000229 & $13 \%$ increase during refeeding \\
\hline D19Wsu162e & chr19:46673783-46718842 & + & 0.00000076 & 0.00073683 & $16 \%$ increase during refeeding \\
\hline Dock6 & chr9:21629974-21634657 & - & 0.00012428 & 0.039252765 & $47 \%$ increase during starvation \\
\hline Ehhadh & chr16:21775584-21787779 & - & 0.0000723 & 0.026440466 & $11 \%$ increase during starvation \\
\hline Eif4h & chr5:135097956-135101235 & - & 0.00040116 & 0.091185016 & $12 \%$ increase during starvation \\
\hline Hnrnpc & chr14:52704066-52723563 & - & 0.00000238 & 0.001782823 & $20 \%$ increase during starvation \\
\hline Huwe1 & chrX:148292456-148294709 & + & $1.09 \mathrm{E}-07$ & 0.00018238 & $12 \%$ increase during refeeding \\
\hline Itch & chr2:154964008-154989128 & + & 0.00000278 & 0.001948704 & $14 \%$ increase during refeeding \\
\hline Itih4 & chr14:31709956-31711042 & + & $3.3 \mathrm{E}-12$ & 1.98E-08 & $11 \%$ increase during starvation \\
\hline Lpin1 & chr12:16575325-16580465 & - & 0.00000125 & 0.001134135 & $19 \%$ increase during starvation \\
\hline Mbnl1 & chr3:60425380-60429045 & + & 0.00000455 & 0.002788029 & $15 \%$ increase during starvation \\
\hline Mbnl1 & chr3:60417442-60419541 & + & 0.0000731 & 0.026440466 & $11 \%$ increase during starvation \\
\hline Mbnl1 & chr3:60419695-60426067 & + & 0.00027537 & 0.07122671 & $12 \%$ increase during starvation \\
\hline MII3 & chr5:24830039-24835058 & - & 0.00029526 & 0.073824331 & $18 \%$ increase during starvation \\
\hline Oma1 & chr4:102998005-103003909 & + & 0.00018758 & 0.054116009 & $12 \%$ increase during refeeding \\
\hline Pex5 & chr6:124353925-124354647 & - & 0.00000163 & 0.001393399 & $11 \%$ increase during refeeding \\
\hline Prpf40a & chr2:53016951-53018591 & - & 0.00000323 & 0.002155621 & $11 \%$ increase during starvation \\
\hline Rbm7 & chr9:48302286-48303259 & - & 0.0001594 & 0.046432076 & $11 \%$ increase during starvation \\
\hline Rtn3 & chr19:7509628-7557428 & - & 0.00000417 & 0.002608786 & $13 \%$ increase during starvation \\
\hline Sec22c & chr9:121604860-121614041 & - & 0.00011677 & 0.038083625 & $40 \%$ increase during starvation \\
\hline Snrnp70 & chr7:52636188-52639173 & - & 0.00029456 & 0.073824331 & $13 \%$ increase during starvation \\
\hline Tm7sf2 & chr19:6067022-6067668 & - & 3.02E-07 & 0.000431301 & $10 \%$ increase during starvation \\
\hline Tmem56 & chr3:120938682-120979905 & - & 0.0000123 & 0.006576559 & $33 \%$ increase during refeeding \\
\hline Tra2a & chr6:49202514-49213836 & - & 0.0000245 & 0.011469045 & $27 \%$ increase during starvation \\
\hline
\end{tabular}




\section{Table 3: Mutually Exclusive Exons}

\section{a. Listed by significance}

\begin{tabular}{|c|c|c|c|c|c|}
\hline Gene Name & $\begin{array}{c}\text { Genomic Location } \\
\text { of Exon }\end{array}$ & Strand & $\boldsymbol{p}$ value & FDR & Frequency of Inclusion \\
\hline Fgfr3 & chr5:34074765-34072983 & + & $5.49 \mathrm{E}-07$ & 0.000623875 & $16 \%$ increase during refeeding \\
\hline Xpo4 & chr14:58267255-58249169 & - & $5.75 \mathrm{E}-06$ & 0.00392477 & $19 \%$ increase during starvation \\
\hline Dock6 & chr9:21633917-21629974 & - & $1.68 \mathrm{E}-05$ & 0.005742965 & $45 \%$ increase during refeeding \\
\hline Setx & chr2:28982393-28981069 & + & 0.000326 & 0.047088463 & $15 \%$ increase during refeeding \\
\hline Zc3h14 & chr12:99990987-99985368 & + & 0.000443 & 0.053974618 & $10 \%$ increase during refeeding \\
\hline Invs & chr4:48397976-48320721 & + & 0.000487 & 0.056392627 & $16 \%$ increase during starvation \\
\hline AK085322 & chr16:86799252-86770642 & - & 0.000704 & 0.070611119 & $15 \%$ increase during refeeding \\
\hline Itch & chr2:154983509-154959321 & + & 0.000784 & 0.076366394 & $14 \%$ increase during starvation \\
\hline Tpst1 & chr5:130610328-130590536 & + & 0.00085 & 0.080488769 & $11 \%$ increase during refeeding \\
\hline Arsg & chr11:109419264-109405142 & + & 0.000954 & 0.087917388 & $12 \%$ increase during refeeding \\
\hline
\end{tabular}

\section{b. Listed alphabetically}

\begin{tabular}{|c|c|c|c|c|c|}
\hline Gene Name & $\begin{array}{c}\text { Genomic Location } \\
\text { of Exon }\end{array}$ & Strand & $\boldsymbol{p}$ value & FDR & Frequency of Inclusion \\
\hline AK085322 & chr16:86799252-86770642 & - & 0.000704 & 0.070611119 & $15 \%$ increase during refeeding \\
\hline Arsg & chr11:109419264-109405142 & + & 0.000954 & 0.087917388 & $12 \%$ increase during refeeding \\
\hline Dock6 & chr9:21633917-21629974 & - & $1.68 \mathrm{E}-05$ & 0.005742965 & $45 \%$ increase during refeeding \\
\hline Fgfr3 & chr5:34074765-34072983 & + & $5.49 \mathrm{E}-07$ & 0.000623875 & $16 \%$ increase during refeeding \\
\hline Invs & chr4:48397976-48320721 & + & 0.000487 & 0.056392627 & $16 \%$ increase during starvation \\
\hline Itch & chr2:154983509-154959321 & + & 0.000784 & 0.076366394 & $14 \%$ increase during starvation \\
\hline Setx & chr2:28982393-28981069 & + & 0.000326 & 0.047088463 & $15 \%$ increase during refeeding \\
\hline Tpst1 & chr5:130610328-130590536 & + & 0.00085 & 0.080488769 & $11 \%$ increase during refeeding \\
\hline Xpo4 & chr14:58267255-58249169 & - & $5.75 \mathrm{E}-06$ & 0.00392477 & $19 \%$ increase during starvation \\
\hline Zc3h14 & chr12:99990987-99985368 & + & 0.000443 & 0.053974618 & $10 \%$ increase during refeeding \\
\hline
\end{tabular}




\section{Table 4: Alternate 3'-splice site usage}

\section{a. Listed by significance}

\begin{tabular}{|c|c|c|c|c|c|}
\hline Gene Name & Genomic Location & Strand & $\boldsymbol{p}$ value & FDR & $\begin{array}{c}\text { Frequency of } \\
\text { Alternate 3'SS Usage }\end{array}$ \\
\hline Itih4 & chr14:31711042-31709956 & + & $6.131 \mathrm{E}-12$ & $1.65 \mathrm{E}-07$ & $14 \%$ increase during starvation \\
\hline Srp19 & chr18:34493946-34491479 & + & $1.254 \mathrm{E}-08$ & 0.000168 & $10 \%$ increase during starvation \\
\hline D19Wsu162e & chr19:46718842-46673783 & + & $4.242 \mathrm{E}-08$ & 0.000456 & $15 \%$ increase during refeeding \\
\hline Eif4a2 & chr16:23110057-23108924 & + & $6.939 \mathrm{E}-07$ & 0.004144 & $10 \%$ increase during refeeding \\
\hline Picalm & chr7:97344164-97340263 & + & $5.563 \mathrm{E}-06$ & 0.023003 & $16 \%$ increase during starvation \\
\hline Gm608 & chr16:44200726-44199134 & + & $1.128 \mathrm{E}-05$ & 0.040418 & $11 \%$ increase during starvation \\
\hline Camk1d & chr2:5366190-5486826 & - & $1.664 \mathrm{E}-05$ & 0.052624 & $26 \%$ increase during starvation \\
\hline Slc17a2 & chr13:23909389-23907142 & + & $2.162 \mathrm{E}-05$ & 0.058111 & $15 \%$ increase during refeeding \\
\hline Itga7 & chr10:128395340-128391127 & + & $2.463 \mathrm{E}-05$ & 0.060176 & $26 \%$ increase during refeeding \\
\hline Slc35b1 & chr11:95247799-95247218 & + & $3.345 \mathrm{E}-05$ & 0.074912 & $10 \%$ increase during refeeding \\
\hline Gss & chr2:155404523-155407769 & - & $4.829 \mathrm{E}-05$ & 0.088095 & $28 \%$ increase during refeeding \\
\hline Nfix & chr8:87251866-87296214 & - & $4.57 \mathrm{E}-05$ & 0.088095 & $10 \%$ increase during refeeding \\
\hline
\end{tabular}

\section{b. Listed alphabetically}

\begin{tabular}{|c|c|c|c|c|c|}
\hline Gene Name & Genomic Location & Strand & p value & FDR & $\begin{array}{c}\text { Frequency of } \\
\text { Alternate 3'SS Usage }\end{array}$ \\
\hline Camk1d & chr2:5366190-5486826 & - & $1.664 \mathrm{E}-05$ & 0.052624 & $26 \%$ increase during starvation \\
\hline D19Wsu162e & chr19:46718842-46673783 & + & $4.242 \mathrm{E}-08$ & 0.000456 & $15 \%$ increase during refeeding \\
\hline Eif4a2 & chr16:23110057-23108924 & + & $6.939 \mathrm{E}-07$ & 0.004144 & $10 \%$ increase during refeeding \\
\hline Gm608 & chr16:44200726-44199134 & + & $1.128 \mathrm{E}-05$ & 0.040418 & $11 \%$ increase during starvation \\
\hline Gss & chr2:155404523-155407769 & - & $4.829 \mathrm{E}-05$ & 0.088095 & $28 \%$ increase during refeeding \\
\hline Itga7 & chr10:128395340-128391127 & + & $2.463 \mathrm{E}-05$ & 0.060176 & $26 \%$ increase during refeeding \\
\hline Itih4 & chr14:31711042-31709956 & + & $6.131 \mathrm{E}-12$ & $1.65 \mathrm{E}-07$ & $14 \%$ increase during starvation \\
\hline Nfix & chr8:87251866-87296214 & - & $4.57 \mathrm{E}-05$ & 0.088095 & $10 \%$ increase during refeeding \\
\hline Picalm & chr7:97344164-97340263 & + & $5.563 \mathrm{E}-06$ & 0.023003 & $16 \%$ increase during starvation \\
\hline Slc17a2 & chr13:23909389-23907142 & + & $2.162 \mathrm{E}-05$ & 0.058111 & $15 \%$ increase during refeeding \\
\hline Slc35b1 & chr11:95247799-95247218 & + & $3.345 \mathrm{E}-05$ & 0.074912 & $10 \%$ increase during refeeding \\
\hline Srp19 & chr18:34493946-34491479 & + & $1.254 \mathrm{E}-08$ & 0.000168 & $10 \%$ increase during starvation \\
\hline
\end{tabular}




\section{Table 5: Alternate 5'-splice site usage}

\section{a. Listed by significance}

\begin{tabular}{|c|c|c|c|c|c|}
\hline Gene Name & Genomic Location & Strand & $p$ value & FDR & $\begin{array}{c}\text { Frequency of } \\
\text { Alternate 5'SS Usage }\end{array}$ \\
\hline Afmid & chr11:117693890-117696166 & + & $6.994 \mathrm{E}-14$ & 4.52E-09 & $13 \%$ increase during refeeding \\
\hline Colec12 & chr18:9720969-9840359 & + & $2.111 \mathrm{E}-07$ & 0.001949 & $13 \%$ increase during refeeding \\
\hline Eif4a2 & chr16:23112530-23114205 & + & 8.988E-07 & 0.004102 & $28 \%$ increase during refeeding \\
\hline Tm7sf2 & chr19:6067668-6067022 & - & 1.049E-06 & 0.004121 & $17 \%$ increase during starvation \\
\hline Epb4.1 & chr4:131513557-131491782 & - & $1.278 \mathrm{E}-06$ & 0.004588 & $21 \%$ increase during refeeding \\
\hline Dido1 & chr2:180426568-180424360 & - & 1.366E-06 & 0.004647 & $13 \%$ increase during starvation \\
\hline 4833439L19Rik & chr13:54665875-54663232 & - & 3.639E-06 & 0.0084 & $13 \%$ increase during starvation \\
\hline Rtn3 & chr19:7530789-7509628 & - & 4.04E-06 & 0.009004 & $11 \%$ increase during starvation \\
\hline Fam53a & chr5:33950979-33950876 & - & $5.735 \mathrm{E}-06$ & 0.011233 & $11 \%$ increase during starvation \\
\hline Prodh2 & chr7:31279543-31291320 & + & $5.606 \mathrm{E}-06$ & 0.011233 & $15 \%$ increase during refeeding \\
\hline Lpin1 & chr12:16580465-16575325 & - & 6.386E-06 & 0.01214 & $22 \%$ increase during starvation \\
\hline Cyp2c70 & chr19:40 & - & 7.502E-06 & 0.013855 & $10 \%$ increase during refeeding \\
\hline Lpin1 & chr12:16596576-16587710 & - & 1.566E-05 & 0.026633 & $10 \%$ increase during starvation \\
\hline Dag1 & chr9:108 & - & $2.595 \mathrm{E}-05$ & 0.040912 & se during starvation \\
\hline Tmem56 & chr3:120983370-120938682 & - & $2.578 \mathrm{E}-05$ & 0.040912 & $33 \%$ increase during refeeding \\
\hline Hnrnpc & chr14:52717689-52704066 & - & 3.472E-05 & 0.052184 & $11 \%$ increase during starvation \\
\hline Rsrc2 & chr5:124199421-124195120 & - & 3.68E-05 & 0.054066 & $10 \%$ increase during starvation \\
\hline Sec31a & chr5:100818086-100815222 & - & 4.872E-05 & 0.067009 & $11 \%$ increase during starvation \\
\hline Bcar3 & chr3:122158010-122207973 & + & 5.99E-05 & 0.075917 & $11 \%$ increase during refeeding \\
\hline Atp13a3 & chr16:30322957-30315987 & - & $6.45 \mathrm{E}-05$ & 0.077201 & $10 \%$ increase during starvation \\
\hline Tra2a & chr6:49214051-49202514 & - & 6.342E-05 & 0.077201 & $20 \%$ increase during starvation \\
\hline Cyp3a25 & chr5:146806440-146804612 & - & 6.672E-05 & 0.078406 & $13 \%$ increase during refeeding \\
\hline
\end{tabular}

\section{b. Listed alphabetically}

\begin{tabular}{|c|c|c|c|c|c|}
\hline Gene Name & Genomic Location & Strand & $p$ value & FDR & $\begin{array}{c}\text { Frequency of } \\
\text { Alternate 5'SS Usage }\end{array}$ \\
\hline 4833439L19Rik & chr13:54665875-54663232 & - & 3.639E-06 & 0.0084 & $13 \%$ increase during starvation \\
\hline Afmid & chr11:117693890-117696166 & + & 6.994E-14 & 4.52E-09 & $13 \%$ increase during refeeding \\
\hline Atp13a3 & chr16:30322957-30315987 & - & $6.45 \mathrm{E}-05$ & 0.077201 & $10 \%$ increase during starvation \\
\hline Bcar3 & chr3:122158010-122207973 & + & 5.99E-05 & 0.075917 & $11 \%$ increase during refeeding \\
\hline Colec12 & chr18:9720969-9840359 & + & $2.111 \mathrm{E}-07$ & 0.001949 & $13 \%$ increase during refeeding \\
\hline Cyp2c70 & chr19:40261776-40261584 & - & 7.502E-06 & 0.013855 & $10 \%$ increase during refeeding \\
\hline Cyp3a25 & chr5:146806440-146804612 & - & 6.672E-05 & 0.078406 & $13 \%$ increase during refeeding \\
\hline Dag1 & chr9:108165955-108155488 & - & $2.595 \mathrm{E}-05$ & 0.040912 & $15 \%$ increase during starvation \\
\hline Dido1 & chr2:180426568-180424360 & - & 1.366E-06 & 0.004647 & $13 \%$ increase during starvation \\
\hline Eif4a2 & chr16:23112530-23114205 & + & 8.988E-07 & 0.004102 & $28 \%$ increase during refeeding \\
\hline Epb4.1 & chr4:131513557-131491782 & - & 1.278E-06 & 0.004588 & $21 \%$ increase during refeeding \\
\hline Fam53a & chr5:33950979-33950876 & - & 5.735E-06 & 0.011233 & $11 \%$ increase during starvation \\
\hline Hnrnpc & chr14:52717689-52704066 & - & 3.472E-05 & 0.052184 & $11 \%$ increase during starvation \\
\hline Lpin1 & chr12:16580465-16575325 & - & 6.386E-06 & 0.01214 & $22 \%$ increase during starvation \\
\hline Lpin1 & chr12:16596576-16587710 & - & 1.566E-05 & 0.026633 & $10 \%$ increase during starvation \\
\hline Prodh2 & chr7:31279543-31291320 & + & 5.606E-06 & 0.011233 & $15 \%$ increase during refeeding \\
\hline Rsrc2 & chr5:124199421-124195120 & - & 3.68E-05 & 0.054066 & $10 \%$ increase during starvation \\
\hline Rtn3 & chr19:7530789-7509628 & - & $4.04 \mathrm{E}-06$ & 0.009004 & $11 \%$ increase during starvation \\
\hline Sec31a & chr5:100818086-100815222 & - & 4.872E-05 & 0.067009 & $11 \%$ increase during starvation \\
\hline Tm7sf2 & chr19:6067668-6067022 & - & 1.049E-06 & 0.004121 & $17 \%$ increase during starvation \\
\hline Tmem56 & chr3:120983370-120938682 & - & $2.578 \mathrm{E}-05$ & 0.040912 & $33 \%$ increase during refeeding \\
\hline Tra2a & chr6:49214051-49202514 & - & 6.342E-05 & 0.077201 & $20 \%$ increase during starvation \\
\hline
\end{tabular}




\section{Table 6: Overall Expression Changes}

\section{a. Listed by significance}

\begin{tabular}{|c|c|c|c|c|c|}
\hline Gene Name & $\log \mathrm{FC}$ & $\log C P M$ & $p$ value & FDR & Absolute Fold Change \\
\hline Pnpla5 & -9.77 & 2.25 & $7.44 \mathrm{E}-105$ & $9.87 \mathrm{E}-101$ & 872.63 fold increase during refeeding \\
\hline Chrna4 & -6.24 & 3.92 & $4.74 \mathrm{E}-103$ & 3.14E-99 & 75.56 fold increase during refeeding \\
\hline Pnpla3 & -5.77 & 5.66 & $1.02 \mathrm{E}-102$ & 4.53E-99 & 54.70 fold increase during refeeding \\
\hline $\mathrm{H} 19$ & 6.16 & 2.87 & $8.31 \mathrm{E}-85$ & $2.76 \mathrm{E}-81$ & 71.28 fold increase during starvation \\
\hline Cyp4a14 & 4.57 & 9.01 & $7.08 \mathrm{E}-82$ & $1.88 \mathrm{E}-78$ & 23.80 fold increase during starvation \\
\hline Fasn & -4.80 & 11.39 & $9.72 \mathrm{E}-81$ & $2.15 \mathrm{E}-77$ & 27.78 fold increase during refeeding \\
\hline Fdps & -4.62 & 8.71 & $1.58 \mathrm{E}-75$ & 3.00E-72 & 24.67 fold increase during refeeding \\
\hline Cyp4a10 & 3.99 & 9.34 & 1.69E-65 & $2.80 \mathrm{E}-62$ & 15.89 fold increase during starvation \\
\hline Sqle & -4.20 & 6.54 & $3.48 E-64$ & $5.13 E-61$ & 18.35 fold increase during refeeding \\
\hline Gpnmb & 4.61 & 3.32 & $2.93 E-62$ & 3.89E-59 & 24.41 fold increase during starvation \\
\hline Vmn2r3 & 7.07 & 0.78 & $4.18 \mathrm{E}-60$ & $5.04 \mathrm{E}-57$ & 134.38 fold increase during starvation \\
\hline Elovl6 & -3.93 & 9.52 & $5.99 \mathrm{E}-60$ & $6.63 \mathrm{E}-57$ & 15.23 fold increase during refeeding \\
\hline Cyp4a31 & 4.36 & 2.92 & 2.99E-59 & $3.06 \mathrm{E}-56$ & 20.59 fold increase during starvation \\
\hline Mvd & -3.99 & 4.25 & 2.87E-56 & $2.72 E-53$ & 15.84 fold increase during refeeding \\
\hline Nsdhl & -3.86 & 5.93 & $5.31 \mathrm{E}-56$ & 4.70E-53 & 14.56 fold increase during refeeding \\
\hline Gm6484 & -3.54 & 6.14 & 3.05E-55 & $2.53 E-52$ & 11.64 fold increase during refeeding \\
\hline Mt2 & 3.80 & 5.43 & 1.74E-53 & $1.28 \mathrm{E}-50$ & 13.89 fold increase during starvation \\
\hline Pcsk9 & -3.70 & 5.78 & 1.67E-53 & $1.28 \mathrm{E}-50$ & 12.99 fold increase during refeeding \\
\hline Fabp5 & -3.52 & 8.05 & 3.69E-51 & $2.58 \mathrm{E}-48$ & 11.45 fold increase during refeeding \\
\hline Nupr1 & 4.15 & 2.69 & $4.88 \mathrm{E}-50$ & $3.24 \mathrm{E}-47$ & 17.78 fold increase during starvation \\
\hline Pdzk1ip1 & -3.71 & 3.01 & $1.18 \mathrm{E}-48$ & $7.48 \mathrm{E}-46$ & 13.09 fold increase during refeeding \\
\hline Acot1 & 3.42 & 5.04 & $1.35 \mathrm{E}-48$ & 8.17E-46 & 10.72 fold increase during starvation \\
\hline A4gnt & -10.36 & -0.32 & $8.03 E-48$ & $4.58 \mathrm{E}-45$ & 1313.34 fold increase during refeeding \\
\hline Cyp51 & -3.53 & 7.62 & $8.28 E-48$ & $4.58 \mathrm{E}-45$ & 11.55 fold increase during refeeding \\
\hline Idi1 & -3.54 & 7.62 & $1.05 \mathrm{E}-47$ & $5.58 \mathrm{E}-45$ & 11.66 fold increase during refeeding \\
\hline Rdh11 & -3.47 & 5.98 & $2.82 E-47$ & $1.44 \mathrm{E}-44$ & 11.06 fold increase during refeeding \\
\hline Acss2 & -3.44 & 9.13 & $1.66 \mathrm{E}-46$ & $8.17 E-44$ & 10.84 fold increase during refeeding \\
\hline Acly & -3.35 & 9.76 & $3.08 E-46$ & $1.46 \mathrm{E}-43$ & 10.20 fold increase during refeeding \\
\hline Me1 & -3.26 & 8.30 & $1.88 \mathrm{E}-43$ & $8.62 E-41$ & 9.59 fold increase during refeeding \\
\hline Acot3 & 3.31 & 3.40 & $1.01 \mathrm{E}-42$ & $4.48 \mathrm{E}-40$ & 9.90 fold increase during starvation \\
\hline Cyp2a4 & 3.38 & 5.30 & $3.20 \mathrm{E}-40$ & 1.37E-37 & 10.41 fold increase during starvation \\
\hline Acaca & -3.02 & 9.58 & $5.18 \mathrm{E}-40$ & $2.15 \mathrm{E}-37$ & 8.10 fold increase during refeeding \\
\hline Gale & -3.10 & 4.08 & 9.77E-40 & 3.93E-37 & 8.58 fold increase during refeeding \\
\hline Acacb & -3.12 & 8.29 & $2.18 \mathrm{E}-39$ & $8.25 \mathrm{E}-37$ & 8.70 fold increase during refeeding \\
\hline Fmr1nb & -5.05 & -0.15 & 2.13E-39 & $8.25 E-37$ & 33.12 fold increase during refeeding \\
\hline Cyp2b9 & 4.01 & 0.99 & $2.28 \mathrm{E}-39$ & 8.39E-37 & 16.13 fold increase during starvation \\
\hline Slc2a4 & -3.14 & 2.03 & 3.92E-38 & 1.40E-35 & 8.82 fold increase during refeeding \\
\hline Pmvk & -3.03 & 5.71 & $4.08 E-38$ & 1.43E-35 & 8.18 fold increase during refeeding \\
\hline Mt1 & 3.08 & 6.33 & 5.39E-38 & 1.84E-35 & 8.47 fold increase during starvation \\
\hline Mid1ip1 & -2.92 & 5.72 & $6.49 E-38$ & $2.15 E-35$ & 7.57 fold increase during refeeding \\
\hline Sc4mol & -3.02 & 7.54 & $8.92 E-37$ & $2.89 \mathrm{E}-34$ & 8.10 fold increase during refeeding \\
\hline Srebf1 & -2.84 & 8.56 & $2.47 E-36$ & $7.80 \mathrm{E}-34$ & 7.14 fold increase during refeeding \\
\hline Hen3 & -3.01 & 4.67 & $2.60 \mathrm{E}-36$ & $8.02 E-34$ & 8.08 fold increase during refeeding \\
\hline $\mathrm{Hmgcr}$ & -2.91 & 8.18 & $2.19 \mathrm{E}-35$ & $6.61 \mathrm{E}-33$ & 7.54 fold increase during refeeding \\
\hline Wfdc3 & 4.87 & -0.09 & $2.68 \mathrm{E}-35$ & $7.90 \mathrm{E}-33$ & 29.14 fold increase during starvation \\
\hline LSS & -2.88 & 6.36 & 1.14E-34 & $3.30 \mathrm{E}-32$ & 7.36 fold increase during refeeding \\
\hline
\end{tabular}




\section{Table 6: Overall Expression Changes (continued)}

\section{a. Listed by significance}

\begin{tabular}{|c|c|c|c|c|c|}
\hline Gene Name & $\log \mathrm{FC}$ & $\log C P M$ & $p$ value & FDR & Absolute Fold Change \\
\hline Pstpip2 & -2.84 & 4.09 & 1.36E-33 & 3.84E-31 & 7.14 fold increase during refeeding \\
\hline Aqp8 & -2.70 & 5.62 & $1.80 \mathrm{E}-32$ & 4.99E-30 & 6.51 fold increase during refeeding \\
\hline Cabp2 & -4.41 & -0.29 & 3.61E-32 & 9.77E-30 & 21.29 fold increase during refeeding \\
\hline Adam11 & 3.32 & 1.30 & $9.82 E-32$ & $2.61 \mathrm{E}-29$ & 10.00 fold increase during starvation \\
\hline Krt23 & 3.18 & 1.77 & 1.27E-31 & 3.30E-29 & 9.07 fold increase during starvation \\
\hline Pklr & -2.70 & 8.77 & 9.53E-31 & $2.43 \mathrm{E}-28$ & 6.48 fold increase during refeeding \\
\hline Cdkn1a & 2.77 & 3.40 & $1.65 \mathrm{E}-30$ & $4.12 \mathrm{E}-28$ & 6.81 fold increase during starvation \\
\hline Thrsp & -2.70 & 10.44 & $2.00 \mathrm{E}-30$ & $4.91 \mathrm{E}-28$ & 6.48 fold increase during refeeding \\
\hline Aacs & -2.58 & 7.51 & $7.22 \mathrm{E}-30$ & $1.74 \mathrm{E}-27$ & 5.98 fold increase during refeeding \\
\hline Sgsm1 & -2.84 & 1.90 & $9.76 \mathrm{E}-30$ & $2.31 \mathrm{E}-27$ & 7.17 fold increase during refeeding \\
\hline Dntt & -2.69 & 2.44 & $1.54 \mathrm{E}-29$ & $3.60 \mathrm{E}-27$ & 6.47 fold increase during refeeding \\
\hline G6pdx & -2.52 & 4.89 & $2.15 E-29$ & $4.93 E-27$ & 5.72 fold increase during refeeding \\
\hline Dhcr7 & -2.52 & 5.10 & $6.62 \mathrm{E}-28$ & $1.49 \mathrm{E}-25$ & 5.74 fold increase during refeeding \\
\hline Ces2b & -3.00 & 0.75 & 7.59E-28 & $1.68 \mathrm{E}-25$ & 8.02 fold increase during refeeding \\
\hline Gm4952 & 2.43 & 5.77 & 8.93E-28 & $1.94 \mathrm{E}-25$ & 5.38 fold increase during starvation \\
\hline Pitx3 & 3.08 & 0.39 & 2.17E-27 & 4.64E-25 & 8.44 fold increase during starvation \\
\hline Insig1 & -2.52 & 8.62 & $4.70 \mathrm{E}-27$ & 9.91E-25 & 5.72 fold increase during refeeding \\
\hline Cyp2b10 & 2.71 & 2.60 & $5.44 \mathrm{E}-27$ & 1.13E-24 & 6.55 fold increase during starvation \\
\hline Ly6d & 3.16 & 1.11 & $7.44 \mathrm{E}-27$ & $1.52 \mathrm{E}-24$ & 8.94 fold increase during starvation \\
\hline Trib3 & 2.78 & 2.55 & $1.30 \mathrm{E}-26$ & $2.61 \mathrm{E}-24$ & 6.86 fold increase during starvation \\
\hline Acat2 & -2.32 & 6.66 & $5.83 E-24$ & $1.15 \mathrm{E}-21$ & 5.00 fold increase during refeeding \\
\hline Acot2 & 2.54 & 2.80 & $8.45 E-24$ & $1.65 \mathrm{E}-21$ & 5.80 fold increase during starvation \\
\hline Vnn1 & 2.20 & 5.92 & $1.04 \mathrm{E}-23$ & $1.99 \mathrm{E}-21$ & 4.60 fold increase during starvation \\
\hline Scd1 & -2.25 & 14.50 & $1.68 \mathrm{E}-23$ & $3.18 \mathrm{E}-21$ & 4.75 fold increase during refeeding \\
\hline Cyp4a32 & 2.22 & 4.13 & $2.26 \mathrm{E}-23$ & $4.22 E-21$ & 4.65 fold increase during starvation \\
\hline Mvk & -2.33 & 4.45 & $2.63 E-23$ & $4.84 \mathrm{E}-21$ & 5.03 fold increase during refeeding \\
\hline Pgd & -2.22 & 4.70 & $3.08 \mathrm{E}-23$ & $5.61 \mathrm{E}-21$ & 4.65 fold increase during refeeding \\
\hline Psrc1 & 4.09 & -0.78 & $4.22 \mathrm{E}-23$ & $7.56 \mathrm{E}-21$ & 17.03 fold increase during starvation \\
\hline Fmo3 & 2.23 & 2.26 & 2.85E-22 & $4.98 \mathrm{E}-20$ & 4.69 fold increase during starvation \\
\hline Myh6 & 3.84 & -0.75 & $2.84 \mathrm{E}-22$ & $4.98 \mathrm{E}-20$ & 14.34 fold increase during starvation \\
\hline Moxd1 & 2.60 & 1.00 & $6.31 E-22$ & $1.09 \mathrm{E}-19$ & 6.08 fold increase during starvation \\
\hline D7Ertd143e & 2.04 & 3.15 & $2.89 \mathrm{E}-21$ & $4.92 \mathrm{E}-19$ & 4.12 fold increase during starvation \\
\hline Mfsd7c & 2.34 & 2.19 & $6.57 \mathrm{E}-21$ & $1.09 \mathrm{E}-18$ & 5.07 fold increase during starvation \\
\hline Osbpl3 & 2.35 & 2.98 & $6.55 E-21$ & $1.09 \mathrm{E}-18$ & 5.11 fold increase during starvation \\
\hline Areg & 4.31 & -1.13 & $6.85 E-21$ & $1.12 \mathrm{E}-18$ & 19.85 fold increase during starvation \\
\hline Eda2r & 2.57 & 1.46 & $2.64 \mathrm{E}-20$ & $4.27 \mathrm{E}-18$ & 5.92 fold increase during starvation \\
\hline Agpat9 & 2.18 & 3.32 & $2.86 \mathrm{E}-20$ & 4.57E-18 & 4.53 fold increase during starvation \\
\hline Fam198a & -2.16 & 2.46 & 4.99E-20 & $7.88 \mathrm{E}-18$ & 4.48 fold increase during refeeding \\
\hline Hapln1 & -2.54 & 0.11 & $1.22 \mathrm{E}-19$ & $1.90 \mathrm{E}-17$ & 5.80 fold increase during refeeding \\
\hline Elovl3 & 1.81 & 6.27 & $2.14 \mathrm{E}-19$ & $3.30 \mathrm{E}-17$ & 3.52 fold increase during starvation \\
\hline Nrg1 & 2.47 & 1.37 & $3.68 \mathrm{E}-19$ & $5.62 \mathrm{E}-17$ & 5.53 fold increase during starvation \\
\hline Fgf21 & 2.41 & 1.99 & 4.30E-19 & $6.48 \mathrm{E}-17$ & 5.32 fold increase during starvation \\
\hline NIrp12 & 1.86 & 5.94 & $6.39 \mathrm{E}-19$ & $9.52 \mathrm{E}-17$ & 3.63 fold increase during starvation \\
\hline Rwdd2a & -2.34 & 1.13 & $6.52 \mathrm{E}-19$ & $9.61 \mathrm{E}-17$ & 5.05 fold increase during refeeding \\
\hline Cd209f & -2.77 & -0.25 & $6.83 \mathrm{E}-19$ & 9.97E-17 & 6.81 fold increase during refeeding \\
\hline Rab30 & 2.03 & 2.88 & $7.40 \mathrm{E}-19$ & $1.07 \mathrm{E}-16$ & 4.08 fold increase during starvation \\
\hline
\end{tabular}




\section{Table 6: Overall Expression Changes (continued)}

\section{a. Listed by significance}

\begin{tabular}{|c|c|c|c|c|c|}
\hline Gene Name & $\log F C$ & $\log C P M$ & $p$ value & FDR & Absolute Fold Change \\
\hline SEPT5 & -2.54 & -0.18 & $1.04 \mathrm{E}-18$ & $1.49 \mathrm{E}-16$ & 5.84 fold increase during refeeding \\
\hline Adcy 10 & -3.23 & -1.01 & $1.43 \mathrm{E}-18$ & $2.02 E-16$ & 9.39 fold increase during refeeding \\
\hline Stard4 & -1.98 & 5.46 & $4.08 \mathrm{E}-18$ & $5.70 \mathrm{E}-16$ & 3.94 fold increase during refeeding \\
\hline Gpam & -1.92 & 8.68 & 5.07E-18 & $7.01 \mathrm{E}-16$ & 3.78 fold increase during refeeding \\
\hline Camk1d & -1.92 & 6.03 & $5.35 \mathrm{E}-18$ & $7.32 \mathrm{E}-16$ & 3.79 fold increase during refeeding \\
\hline Cidec & 2.10 & 2.84 & $6.47 \mathrm{E}-18$ & $8.76 \mathrm{E}-16$ & 4.30 fold increase during starvation \\
\hline Hsd17b6 & 2.00 & 6.49 & $1.38 \mathrm{E}-17$ & $1.84 \mathrm{E}-15$ & 4.01 fold increase during starvation \\
\hline Lgals4 & 1.98 & 2.91 & $2.24 \mathrm{E}-17$ & $2.97 \mathrm{E}-15$ & 3.94 fold increase during starvation \\
\hline Egr1 & 2.25 & 2.05 & $2.52 \mathrm{E}-17$ & $3.31 \mathrm{E}-15$ & 4.76 fold increase during starvation \\
\hline Gstm2 & -1.79 & 5.61 & $2.92 \mathrm{E}-17$ & $3.80 \mathrm{E}-15$ & 3.47 fold increase during refeeding \\
\hline Mmp12 & 2.26 & 2.70 & $3.91 \mathrm{E}-17$ & $5.04 \mathrm{E}-15$ & 4.80 fold increase during starvation \\
\hline Sc5d & -1.86 & 8.50 & $4.39 \mathrm{E}-17$ & $5.60 \mathrm{E}-15$ & 3.64 fold increase during refeeding \\
\hline Gck & -1.85 & 8.08 & $5.98 \mathrm{E}-17$ & $7.56 \mathrm{E}-15$ & 3.62 fold increase during refeeding \\
\hline Ubd & 2.41 & 0.93 & $8.12 \mathrm{E}-17$ & $1.02 E-14$ & 5.33 fold increase during starvation \\
\hline Fdft1 & -1.85 & 6.88 & $3.56 \mathrm{E}-16$ & $4.42 E-14$ & 3.59 fold increase during refeeding \\
\hline Sorcs2 & -2.60 & -0.40 & $3.71 \mathrm{E}-16$ & $4.56 \mathrm{E}-14$ & 6.08 fold increase during refeeding \\
\hline Serpina5 & -2.11 & 0.94 & $4.93 \mathrm{E}-16$ & $6.00 \mathrm{E}-14$ & 4.31 fold increase during refeeding \\
\hline Arl5c & -1.93 & 1.44 & $5.16 \mathrm{E}-16$ & $6.23 E-14$ & 3.81 fold increase during refeeding \\
\hline Cerkl & 2.24 & 0.02 & $9.90 \mathrm{E}-16$ & $1.18 \mathrm{E}-13$ & 4.73 fold increase during starvation \\
\hline Ugt1a5 & -1.80 & 4.93 & $1.43 \mathrm{E}-15$ & $1.70 \mathrm{E}-13$ & 3.48 fold increase during refeeding \\
\hline Acaa1b & 1.75 & 9.03 & $1.67 \mathrm{E}-15$ & $1.96 \mathrm{E}-13$ & 3.36 fold increase during starvation \\
\hline Ehhadh & 1.78 & 8.48 & $1.78 \mathrm{E}-15$ & $2.08 \mathrm{E}-13$ & 3.44 fold increase during starvation \\
\hline Khk & -1.80 & 8.60 & $1.81 \mathrm{E}-15$ & $2.09 \mathrm{E}-13$ & 3.48 fold increase during refeeding \\
\hline Insig2 & 1.65 & 7.25 & $1.88 \mathrm{E}-15$ & $2.15 \mathrm{E}-13$ & 3.14 fold increase during starvation \\
\hline Trem2 & 2.31 & 1.01 & $2.03 E-15$ & $2.31 \mathrm{E}-13$ & 4.96 fold increase during starvation \\
\hline Acmsd & -1.66 & 5.50 & $2.40 \mathrm{E}-15$ & $2.70 \mathrm{E}-13$ & 3.16 fold increase during refeeding \\
\hline Slc27a1 & 1.78 & 2.90 & $2.62 \mathrm{E}-15$ & $2.92 E-13$ & 3.43 fold increase during starvation \\
\hline Gpi1 & -1.73 & 7.86 & $2.92 \mathrm{E}-15$ & $3.23 E-13$ & 3.32 fold increase during refeeding \\
\hline Arntl & 1.72 & 2.62 & $3.26 \mathrm{E}-15$ & $3.57 \mathrm{E}-13$ & 3.31 fold increase during starvation \\
\hline Lbp & 1.70 & 5.65 & $3.79 \mathrm{E}-15$ & $4.12 \mathrm{E}-13$ & 3.25 fold increase during starvation \\
\hline Rnd2 & -1.93 & 1.54 & $3.95 \mathrm{E}-15$ & $4.26 \mathrm{E}-13$ & 3.81 fold increase during refeeding \\
\hline $\operatorname{lgfbp} 1$ & 1.65 & 6.77 & $4.45 \mathrm{E}-15$ & $4.76 \mathrm{E}-13$ & 3.14 fold increase during starvation \\
\hline Clec7a & 2.00 & 4.14 & $5.34 \mathrm{E}-15$ & $5.67 \mathrm{E}-13$ & 4.00 fold increase during starvation \\
\hline Akr1b7 & 2.51 & 0.16 & $6.99 \mathrm{E}-15$ & $7.37 \mathrm{E}-13$ & 5.69 fold increase during starvation \\
\hline Slc17a4 & -1.68 & 5.25 & $7.34 \mathrm{E}-15$ & $7.67 \mathrm{E}-13$ & 3.20 fold increase during refeeding \\
\hline Prss8 & -1.75 & 3.06 & $8.62 \mathrm{E}-15$ & $8.94 \mathrm{E}-13$ & 3.37 fold increase during refeeding \\
\hline Gadd45g & 1.59 & 4.95 & $1.00 \mathrm{E}-14$ & $1.03 \mathrm{E}-12$ & 3.00 fold increase during starvation \\
\hline Rab11fip4 & 1.94 & 1.35 & 1.63E-14 & $1.65 \mathrm{E}-12$ & 3.84 fold increase during starvation \\
\hline Tnnc1 & -2.22 & -0.16 & $1.62 \mathrm{E}-14$ & $1.65 \mathrm{E}-12$ & 4.67 fold increase during refeeding \\
\hline Atp2b2 & -1.73 & 1.66 & $2.08 \mathrm{E}-14$ & $2.09 \mathrm{E}-12$ & 3.31 fold increase during refeeding \\
\hline Hapln4 & -1.83 & 1.43 & $2.15 \mathrm{E}-14$ & $2.15 \mathrm{E}-12$ & 3.55 fold increase during refeeding \\
\hline Slc22a5 & 1.65 & 4.81 & $2.64 \mathrm{E}-14$ & $2.61 \mathrm{E}-12$ & 3.13 fold increase during starvation \\
\hline Gucy2c & 2.35 & 0.02 & $2.70 \mathrm{E}-14$ & $2.63 \mathrm{E}-12$ & 5.08 fold increase during starvation \\
\hline Snhg11 & -1.91 & 1.39 & $2.69 \mathrm{E}-14$ & $2.63 E-12$ & 3.76 fold increase during refeeding \\
\hline Hes6 & -1.71 & 6.40 & $3.02 \mathrm{E}-14$ & $2.92 \mathrm{E}-12$ & 3.28 fold increase during refeeding \\
\hline Gabbr2 & -1.67 & 4.59 & $3.13 \mathrm{E}-14$ & $3.01 \mathrm{E}-12$ & 3.18 fold increase during refeeding \\
\hline
\end{tabular}




\section{Table 6: Overall Expression Changes (continued)}

\section{a. Listed by significance}

\begin{tabular}{|c|c|c|c|c|c|}
\hline Gene Name & $\log F C$ & $\log C P M$ & $p$ value & FDR & Absolute Fold Change \\
\hline Ttc25 & -2.53 & -0.86 & $3.16 \mathrm{E}-14$ & $3.02 \mathrm{E}-12$ & 5.77 fold increase during refeeding \\
\hline Ccdc69 & -2.24 & -0.25 & 3.33E-14 & $3.16 \mathrm{E}-12$ & 4.73 fold increase during refeeding \\
\hline Fam81a & 2.06 & 0.79 & $5.84 \mathrm{E}-14$ & $5.50 \mathrm{E}-12$ & 4.17 fold increase during starvation \\
\hline Aldoc & -1.70 & 3.93 & $1.02 \mathrm{E}-13$ & $9.50 \mathrm{E}-12$ & 3.25 fold increase during refeeding \\
\hline Fam107a & 1.55 & 3.96 & $1.32 \mathrm{E}-13$ & $1.22 \mathrm{E}-11$ & 2.92 fold increase during starvation \\
\hline Eln & 1.95 & 1.67 & $1.45 \mathrm{E}-13$ & $1.33 \mathrm{E}-11$ & 3.87 fold increase during starvation \\
\hline Fbxo32 & 1.78 & 2.17 & $1.77 \mathrm{E}-13$ & $1.62 \mathrm{E}-11$ & 3.43 fold increase during starvation \\
\hline Hsbp1I1 & -1.76 & 1.48 & $2.75 \mathrm{E}-13$ & $2.50 \mathrm{E}-11$ & 3.39 fold increase during refeeding \\
\hline C330021F23Rik & -1.98 & 0.14 & $3.63 E-13$ & $3.28 \mathrm{E}-11$ & 3.94 fold increase during refeeding \\
\hline $\mathrm{Nfe} 2$ & -2.15 & -0.35 & $4.01 \mathrm{E}-13$ & $3.57 \mathrm{E}-11$ & 4.43 fold increase during refeeding \\
\hline Slc10a2 & -1.58 & 4.17 & $4.01 \mathrm{E}-13$ & $3.57 \mathrm{E}-11$ & 2.99 fold increase during refeeding \\
\hline Cd83 & 2.00 & 1.06 & $4.31 \mathrm{E}-13$ & $3.81 \mathrm{E}-11$ & 4.00 fold increase during starvation \\
\hline Sec61g & -1.56 & 5.98 & $4.36 \mathrm{E}-13$ & $3.83 E-11$ & 2.96 fold increase during refeeding \\
\hline Psat1 & -1.47 & 2.29 & $4.56 \mathrm{E}-13$ & $3.98 \mathrm{E}-11$ & 2.76 fold increase during refeeding \\
\hline 1600016N20Rik & -1.85 & 0.68 & $4.70 \mathrm{E}-13$ & 4.07E-11 & 3.60 fold increase during refeeding \\
\hline Tcp11l2 & 1.53 & 4.10 & $5.10 \mathrm{E}-13$ & $4.40 \mathrm{E}-11$ & 2.89 fold increase during starvation \\
\hline Fabp7 & 1.92 & 1.00 & $5.33 \mathrm{E}-13$ & $4.57 \mathrm{E}-11$ & 3.80 fold increase during starvation \\
\hline Mmp2 & 1.81 & 3.01 & $5.58 \mathrm{E}-13$ & 4.75E-11 & 3.52 fold increase during starvation \\
\hline Lrg1 & 1.51 & 7.28 & $6.78 \mathrm{E}-13$ & $5.73 \mathrm{E}-11$ & 2.84 fold increase during starvation \\
\hline Sec61b & -1.55 & 5.84 & $7.48 \mathrm{E}-13$ & $6.28 \mathrm{E}-11$ & 2.93 fold increase during refeeding \\
\hline Fras1 & -1.63 & 3.75 & $7.68 \mathrm{E}-13$ & $6.41 \mathrm{E}-11$ & 3.09 fold increase during refeeding \\
\hline Cyp2a22 & 1.78 & 2.79 & 9.35E-13 & $7.75 \mathrm{E}-11$ & 3.45 fold increase during starvation \\
\hline Rec8 & -1.89 & 0.50 & $9.82 \mathrm{E}-13$ & $8.10 \mathrm{E}-11$ & 3.70 fold increase during refeeding \\
\hline Fam89a & 1.86 & 0.39 & $9.90 \mathrm{E}-13$ & $8.11 \mathrm{E}-11$ & 3.63 fold increase during starvation \\
\hline Fads3 & -1.53 & 4.72 & $1.61 \mathrm{E}-12$ & $1.30 \mathrm{E}-10$ & 2.89 fold increase during refeeding \\
\hline $\mathrm{Rfx} 4$ & 1.86 & -0.04 & $1.61 \mathrm{E}-12$ & $1.30 \mathrm{E}-10$ & 3.62 fold increase during starvation \\
\hline Dak & -1.59 & 7.84 & $2.20 \mathrm{E}-12$ & 1.77E-10 & 3.00 fold increase during refeeding \\
\hline Sucnr1 & -1.60 & 4.44 & $2.32 \mathrm{E}-12$ & $1.86 \mathrm{E}-10$ & 3.03 fold increase during refeeding \\
\hline Sh3pxd2b & 1.80 & 2.12 & $2.52 \mathrm{E}-12$ & $2.00 \mathrm{E}-10$ & 3.47 fold increase during starvation \\
\hline Utp14b & -1.54 & 4.26 & $3.96 \mathrm{E}-12$ & $3.13 \mathrm{E}-10$ & 2.90 fold increase during refeeding \\
\hline Afmid & 1.48 & 7.10 & $4.23 \mathrm{E}-12$ & $3.33 \mathrm{E}-10$ & 2.79 fold increase during starvation \\
\hline Osbp2 & 2.46 & -0.86 & $4.28 \mathrm{E}-12$ & $3.34 \mathrm{E}-10$ & 5.51 fold increase during starvation \\
\hline Echdc1 & -1.54 & 4.43 & $5.96 \mathrm{E}-12$ & $4.62 \mathrm{E}-10$ & 2.92 fold increase during refeeding \\
\hline Cyp3a59 & 1.63 & 4.77 & $7.08 \mathrm{E}-12$ & $5.46 \mathrm{E}-10$ & 3.09 fold increase during starvation \\
\hline Mycn & -1.69 & 1.68 & $7.49 \mathrm{E}-12$ & $5.75 \mathrm{E}-10$ & 3.22 fold increase during refeeding \\
\hline Gnat2 & -1.71 & 0.33 & $7.56 \mathrm{E}-12$ & $5.77 \mathrm{E}-10$ & 3.28 fold increase during refeeding \\
\hline Slc27a2 & 1.46 & 10.55 & $1.05 \mathrm{E}-11$ & $7.95 \mathrm{E}-10$ & 2.74 fold increase during starvation \\
\hline Scube1 & 1.93 & 0.59 & $1.07 \mathrm{E}-11$ & $8.04 \mathrm{E}-10$ & 3.81 fold increase during starvation \\
\hline Dbi & -1.44 & 8.59 & $1.41 \mathrm{E}-11$ & 1.06E-09 & 2.71 fold increase during refeeding \\
\hline Ccdc120 & 1.96 & 0.47 & $1.42 \mathrm{E}-11$ & 1.06E-09 & 3.89 fold increase during starvation \\
\hline Tkt & -1.49 & 7.40 & $1.50 \mathrm{E}-11$ & $1.11 \mathrm{E}-09$ & 2.81 fold increase during refeeding \\
\hline Pmm1 & 1.84 & 0.74 & $1.63 \mathrm{E}-11$ & 1.20E-09 & 3.58 fold increase during starvation \\
\hline Arrdc3 & -1.42 & 7.23 & $1.76 \mathrm{E}-11$ & 1.29E-09 & 2.67 fold increase during refeeding \\
\hline Slc7a4 & -1.62 & 0.90 & $2.19 \mathrm{E}-11$ & 1.60E-09 & 3.07 fold increase during refeeding \\
\hline Slc25a34 & 1.49 & 3.01 & $2.89 \mathrm{E}-11$ & 2.10E-09 & 2.80 fold increase during starvation \\
\hline 0610007P14Rik & -1.51 & 5.33 & $3.28 \mathrm{E}-11$ & 2.36E-09 & 2.85 fold increase during refeeding \\
\hline
\end{tabular}




\section{Table 6: Overall Expression Changes (continued)}

\section{a. Listed by significance}

\begin{tabular}{|c|c|c|c|c|c|}
\hline Gene Name & $\log F C$ & $\log C P M$ & $p$ value & FDR & Absolute Fold Change \\
\hline Pla2g12a & 1.53 & 3.74 & $3.51 \mathrm{E}-11$ & 2.52E-09 & 2.88 fold increase during starvation \\
\hline Afp & -1.55 & 1.26 & $4.12 \mathrm{E}-11$ & 2.94E-09 & 2.94 fold increase during refeeding \\
\hline Cd300lf & 1.77 & 0.41 & $4.48 \mathrm{E}-11$ & 3.18E-09 & 3.42 fold increase during starvation \\
\hline Lpl & 1.66 & 4.64 & 4.54E-11 & 3.20E-09 & 3.16 fold increase during starvation \\
\hline Aqp7 & 2.72 & -1.19 & $4.92 \mathrm{E}-11$ & 3.45E-09 & 6.60 fold increase during starvation \\
\hline $\mathrm{Pla} 2 \mathrm{~g} 7$ & 1.55 & 2.86 & $5.15 \mathrm{E}-11$ & 3.59E-09 & 2.94 fold increase during starvation \\
\hline Slc22a28 & -1.58 & 2.56 & $5.17 \mathrm{E}-11$ & 3.59E-09 & 2.99 fold increase during refeeding \\
\hline Fkbp11 & -1.40 & 3.85 & $5.34 \mathrm{E}-11$ & 3.69E-09 & 2.63 fold increase during refeeding \\
\hline Nr1i3 & 1.36 & 7.05 & $5.46 \mathrm{E}-11$ & 3.76E-09 & 2.56 fold increase during starvation \\
\hline 4930452B06Rik & -1.45 & 2.22 & $8.87 \mathrm{E}-11$ & $6.03 E-09$ & 2.74 fold increase during refeeding \\
\hline Lamb3 & 1.42 & 2.24 & $8.85 \mathrm{E}-11$ & 6.03E-09 & 2.68 fold increase during starvation \\
\hline Sell & 1.70 & 0.69 & $8.90 \mathrm{E}-11$ & 6.03E-09 & 3.26 fold increase during starvation \\
\hline Acsl3 & -1.46 & 5.00 & $8.98 \mathrm{E}-11$ & 6.05E-09 & 2.75 fold increase during refeeding \\
\hline Gstm6 & -1.42 & 6.18 & $1.09 \mathrm{E}-10$ & 7.33E-09 & 2.68 fold increase during refeeding \\
\hline 3930402G23Rik & 2.21 & -1.07 & $1.15 \mathrm{E}-10$ & 7.66E-09 & 4.64 fold increase during starvation \\
\hline Hsd17b7 & -1.50 & 6.45 & $1.26 \mathrm{E}-10$ & 8.35E-09 & 2.82 fold increase during refeeding \\
\hline Cd300lb & 1.80 & 0.68 & $1.44 \mathrm{E}-10$ & 9.52E-09 & 3.48 fold increase during starvation \\
\hline Klk1b4 & -1.48 & 1.61 & $1.51 \mathrm{E}-10$ & 9.92E-09 & 2.79 fold increase during refeeding \\
\hline Acsl5 & -1.39 & 9.12 & $1.52 \mathrm{E}-10$ & 9.94E-09 & 2.63 fold increase during refeeding \\
\hline Cyp1b1 & 1.70 & 0.99 & $1.77 \mathrm{E}-10$ & $1.15 \mathrm{E}-08$ & 3.24 fold increase during starvation \\
\hline N28178 & 2.07 & -0.63 & $1.97 \mathrm{E}-10$ & 1.27E-08 & 4.20 fold increase during starvation \\
\hline Gpr110 & -1.45 & 4.25 & $2.06 \mathrm{E}-10$ & 1.32E-08 & 2.73 fold increase during refeeding \\
\hline Haus7 & -1.40 & 3.42 & $2.06 \mathrm{E}-10$ & $1.32 \mathrm{E}-08$ & 2.64 fold increase during refeeding \\
\hline Cux2 & 1.75 & -0.22 & $2.10 \mathrm{E}-10$ & 1.34E-08 & 3.37 fold increase during starvation \\
\hline Eci2 & 1.36 & 7.83 & $2.60 \mathrm{E}-10$ & 1.63E-08 & 2.56 fold increase during starvation \\
\hline Gm6614 & 1.75 & 0.38 & $2.60 \mathrm{E}-10$ & $1.63 \mathrm{E}-08$ & 3.35 fold increase during starvation \\
\hline Por & 1.34 & 9.06 & $2.69 \mathrm{E}-10$ & 1.69E-08 & 2.54 fold increase during starvation \\
\hline Ttbk1 & -1.76 & 0.42 & $2.97 \mathrm{E}-10$ & $1.85 \mathrm{E}-08$ & 3.38 fold increase during refeeding \\
\hline Acot11 & -1.50 & 3.66 & $3.83 E-10$ & 2.36E-08 & 2.83 fold increase during refeeding \\
\hline Cyp3a11 & 1.47 & 10.61 & $3.96 \mathrm{E}-10$ & $2.44 \mathrm{E}-08$ & 2.78 fold increase during starvation \\
\hline Rasal1 & 2.20 & -0.93 & $4.28 \mathrm{E}-10$ & $2.62 \mathrm{E}-08$ & 4.60 fold increase during starvation \\
\hline Ihh & -1.42 & 1.51 & $4.43 \mathrm{E}-10$ & $2.70 \mathrm{E}-08$ & 2.68 fold increase during refeeding \\
\hline Fads1 & -1.35 & 9.19 & $4.49 \mathrm{E}-10$ & $2.72 \mathrm{E}-08$ & 2.55 fold increase during refeeding \\
\hline Hspa1a & -1.45 & 2.07 & $4.54 \mathrm{E}-10$ & $2.74 \mathrm{E}-08$ & 2.74 fold increase during refeeding \\
\hline Akr1c18 & -1.69 & -0.11 & $4.91 \mathrm{E}-10$ & 2.95E-08 & 3.23 fold increase during refeeding \\
\hline Cxcr4 & 1.71 & 1.14 & $5.67 \mathrm{E}-10$ & 3.39E-08 & 3.28 fold increase during starvation \\
\hline Fam187b & -1.36 & 2.49 & $6.11 \mathrm{E}-10$ & $3.62 \mathrm{E}-08$ & 2.56 fold increase during refeeding \\
\hline Adamts15 & 1.46 & 1.64 & $6.92 E-10$ & 4.08E-08 & 2.75 fold increase during starvation \\
\hline Aldob & -1.37 & 13.03 & $7.21 \mathrm{E}-10$ & $4.23 E-08$ & 2.58 fold increase during refeeding \\
\hline Gdf15 & 1.76 & 1.33 & $7.38 \mathrm{E}-10$ & 4.32E-08 & 3.38 fold increase during starvation \\
\hline Gbe1 & -1.36 & 7.94 & $8.85 \mathrm{E}-10$ & $5.15 \mathrm{E}-08$ & 2.57 fold increase during refeeding \\
\hline A330069E16Rik & -1.74 & -0.48 & $9.10 \mathrm{E}-10$ & 5.27E-08 & 3.35 fold increase during refeeding \\
\hline Odf3b & 1.44 & 1.53 & $9.58 \mathrm{E}-10$ & 5.53E-08 & 2.72 fold increase during starvation \\
\hline Clec2h & 1.36 & 2.70 & 1.22E-09 & 7.00E-08 & 2.57 fold increase during starvation \\
\hline Gnat1 & -1.35 & 2.35 & 1.27E-09 & 7.27E-08 & 2.56 fold increase during refeeding \\
\hline Frat1 & 1.33 & 2.44 & $1.28 \mathrm{E}-09$ & $7.28 \mathrm{E}-08$ & 2.52 fold increase during starvation \\
\hline
\end{tabular}




\section{Table 6: Overall Expression Changes (continued)}

\section{a. Listed by significance}

\begin{tabular}{|c|c|c|c|c|c|}
\hline Gene Name & $\log F C$ & $\log C P M$ & $p$ value & FDR & Absolute Fold Change \\
\hline 9330182L06Rik & -1.82 & -0.47 & 1.37E-09 & 7.76E-08 & 3.54 fold increase during refeeding \\
\hline Cd63 & 1.64 & 1.73 & 1.47E-09 & 8.30E-08 & 3.13 fold increase during starvation \\
\hline Cyb5r3 & -1.33 & 8.86 & 2.14E-09 & $1.20 \mathrm{E}-07$ & 2.51 fold increase during refeeding \\
\hline Col15a1 & -1.32 & 2.45 & 2.22E-09 & 1.23E-07 & 2.50 fold increase during refeeding \\
\hline Islr & 1.53 & 1.74 & 2.22E-09 & $1.23 \mathrm{E}-07$ & 2.88 fold increase during starvation \\
\hline Ttn & 1.47 & 2.36 & $2.21 \mathrm{E}-09$ & 1.23E-07 & 2.76 fold increase during starvation \\
\hline Aldh1l2 & -1.54 & 0.18 & 2.30E-09 & 1.27E-07 & 2.91 fold increase during refeeding \\
\hline Acpp & -1.44 & 3.19 & 2.39E-09 & $1.31 \mathrm{E}-07$ & 2.71 fold increase during refeeding \\
\hline Rap1gap2 & 1.54 & 1.63 & 2.49E-09 & 1.36E-07 & 2.91 fold increase during starvation \\
\hline Htra3 & 1.60 & 1.35 & 2.70E-09 & 1.47E-07 & 3.03 fold increase during starvation \\
\hline Ldlr & -1.35 & 7.39 & 3.05E-09 & 1.65E-07 & 2.55 fold increase during refeeding \\
\hline Mmp27 & 1.58 & 0.67 & 3.15E-09 & $1.70 \mathrm{E}-07$ & 3.00 fold increase during starvation \\
\hline Lhx6 & -1.39 & 2.05 & 3.17E-09 & $1.70 \mathrm{E}-07$ & 2.62 fold increase during refeeding \\
\hline Rsad2 & 1.37 & 3.50 & 3.36E-09 & 1.79E-07 & 2.59 fold increase during starvation \\
\hline Ralgps1 & -1.35 & 2.04 & 3.97E-09 & $2.11 \mathrm{E}-07$ & 2.55 fold increase during refeeding \\
\hline Prokr1 & 2.27 & -1.34 & 4.02E-09 & $2.13 \mathrm{E}-07$ & 4.82 fold increase during starvation \\
\hline Uap1I1 & 1.51 & 3.14 & 4.25E-09 & $2.24 \mathrm{E}-07$ & 2.85 fold increase during starvation \\
\hline Sema3g & 2.00 & -0.92 & 6.91E-09 & $3.58 \mathrm{E}-07$ & 4.01 fold increase during starvation \\
\hline Gm6907 & 1.40 & 1.06 & 9.78E-09 & 4.97E-07 & 2.64 fold increase during starvation \\
\hline Adamts12 & 1.60 & 0.95 & $1.24 \mathrm{E}-08$ & $6.20 \mathrm{E}-07$ & 3.02 fold increase during starvation \\
\hline E2f2 & -1.35 & 0.23 & $1.50 \mathrm{E}-08$ & $7.46 \mathrm{E}-07$ & 2.54 fold increase during refeeding \\
\hline 9530026P05Rik & 1.46 & 0.91 & $1.52 \mathrm{E}-08$ & $7.50 \mathrm{E}-07$ & 2.76 fold increase during starvation \\
\hline Serpinb1a & 1.71 & -0.41 & $1.80 \mathrm{E}-08$ & $8.81 \mathrm{E}-07$ & 3.28 fold increase during starvation \\
\hline II1rn & 1.60 & 0.91 & $1.82 \mathrm{E}-08$ & $8.86 \mathrm{E}-07$ & 3.03 fold increase during starvation \\
\hline Slc41a3 & 1.40 & 2.65 & 1.85E-08 & 8.95E-07 & 2.64 fold increase during starvation \\
\hline Themis & 1.69 & -0.74 & 2.08E-08 & 9.99E-07 & 3.23 fold increase during starvation \\
\hline Wdr52 & 1.95 & -1.06 & 3.20E-08 & $1.51 \mathrm{E}-06$ & 3.86 fold increase during starvation \\
\hline Gpr35 & 1.45 & 0.74 & 3.52E-08 & 1.64E-06 & 2.74 fold increase during starvation \\
\hline Gdpd1 & 1.49 & 0.82 & 3.86E-08 & $1.79 \mathrm{E}-06$ & 2.82 fold increase during starvation \\
\hline BC057022 & 1.40 & 0.24 & 4.00E-08 & 1.83E-06 & 2.64 fold increase during starvation \\
\hline $\operatorname{ltgax}$ & 1.48 & 1.85 & 4.17E-08 & 1.90E-06 & 2.80 fold increase during starvation \\
\hline Frat2 & 1.41 & 0.59 & 4.53E-08 & $2.04 \mathrm{E}-06$ & 2.65 fold increase during starvation \\
\hline Dkk3 & 1.42 & 1.86 & 6.33E-08 & $2.77 E-06$ & 2.68 fold increase during starvation \\
\hline Naip1 & 1.36 & 0.71 & 8.64E-08 & 3.71E-06 & 2.56 fold increase during starvation \\
\hline $\mathrm{Bcl} 2 \mathrm{a} 1 \mathrm{~b}$ & 1.56 & 0.14 & 9.09E-08 & $3.88 \mathrm{E}-06$ & 2.95 fold increase during starvation \\
\hline Thbs2 & 1.32 & 3.27 & 9.37E-08 & 3.98E-06 & 2.50 fold increase during starvation \\
\hline Rgs1 & 1.37 & 1.21 & $1.05 \mathrm{E}-07$ & 4.43E-06 & 2.58 fold increase during starvation \\
\hline Ptpro & 1.50 & -0.13 & $1.21 \mathrm{E}-07$ & 5.03E-06 & 2.83 fold increase during starvation \\
\hline B4galnt3 & -1.40 & 0.69 & 1.62E-07 & $6.61 \mathrm{E}-06$ & 2.63 fold increase during refeeding \\
\hline Neto2 & 1.41 & 0.85 & 1.66E-07 & $6.72 \mathrm{E}-06$ & 2.66 fold increase during starvation \\
\hline Gldn & -1.39 & -0.24 & 1.89E-07 & 7.58E-06 & 2.62 fold increase during refeeding \\
\hline Myom3 & 1.39 & 0.05 & 1.93E-07 & 7.70E-06 & 2.61 fold increase during starvation \\
\hline $\mathrm{IITr}$ & 1.56 & -0.15 & $2.24 \mathrm{E}-07$ & 8.86E-06 & 2.95 fold increase during starvation \\
\hline Arhgap22 & 1.68 & -0.45 & $2.38 \mathrm{E}-07$ & 9.38E-06 & 3.21 fold increase during starvation \\
\hline Ube2c & 1.59 & -0.77 & 3.98E-07 & $1.52 \mathrm{E}-05$ & 3.02 fold increase during starvation \\
\hline Dnahc17 & -1.41 & -0.08 & $4.40 \mathrm{E}-07$ & 1.64E-05 & 2.65 fold increase during refeeding \\
\hline
\end{tabular}




\section{Table 6: Overall Expression Changes (continued)}

\section{a. Listed by significance}

\begin{tabular}{|c|c|c|c|c|c|}
\hline Gene Name & $\log F C$ & $\log C P M$ & $p$ value & FDR & Absolute Fold Change \\
\hline Icosl & 1.32 & 0.85 & 4.73E-07 & 1.75E-05 & 2.50 fold increase during starvation \\
\hline Snurf & -1.53 & -0.83 & 4.73E-07 & $1.75 \mathrm{E}-05$ & 2.88 fold increase during refeeding \\
\hline Adam8 & 1.60 & -0.07 & 4.75E-07 & 1.75E-05 & 3.03 fold increase during starvation \\
\hline $\mathrm{H} 2-\mathrm{BI}$ & -1.44 & -0.39 & $5.98 \mathrm{E}-07$ & $2.16 \mathrm{E}-05$ & 2.71 fold increase during refeeding \\
\hline Btla & 1.33 & 0.82 & $6.43 \mathrm{E}-07$ & $2.30 \mathrm{E}-05$ & 2.52 fold increase during starvation \\
\hline Amz1 & 1.41 & 0.22 & $6.50 \mathrm{E}-07$ & $2.32 \mathrm{E}-05$ & 2.66 fold increase during starvation \\
\hline Tctex1d4 & 1.38 & -0.63 & 8.84E-07 & 3.09E-05 & 2.60 fold increase during starvation \\
\hline Slc13a5 & 1.38 & 0.45 & $1.00 \mathrm{E}-06$ & $3.45 \mathrm{E}-05$ & 2.61 fold increase during starvation \\
\hline Spink3 & 1.74 & -0.72 & $1.04 \mathrm{E}-06$ & $3.55 \mathrm{E}-05$ & 3.35 fold increase during starvation \\
\hline Prrg4 & 1.40 & 0.54 & $1.09 \mathrm{E}-06$ & $3.70 \mathrm{E}-05$ & 2.63 fold increase during starvation \\
\hline Bmper & 1.51 & -0.01 & 1.17E-06 & 3.96E-05 & 2.85 fold increase during starvation \\
\hline Cilp & 1.56 & -0.61 & $1.43 \mathrm{E}-06$ & 4.71E-05 & 2.94 fold increase during starvation \\
\hline Rad51I1 & 1.48 & 0.13 & 1.47E-06 & 4.82E-05 & 2.78 fold increase during starvation \\
\hline Odz4 & 1.50 & -0.23 & $1.55 \mathrm{E}-06$ & 4.99E-05 & 2.83 fold increase during starvation \\
\hline Magi2 & 1.85 & -1.41 & $1.70 \mathrm{E}-06$ & $5.46 \mathrm{E}-05$ & 3.60 fold increase during starvation \\
\hline Elovl7 & 1.43 & 0.49 & $1.86 \mathrm{E}-06$ & 5.93E-05 & 2.70 fold increase during starvation \\
\hline Cd19 & 1.86 & -1.40 & $2.13 \mathrm{E}-06$ & $6.72 \mathrm{E}-05$ & 3.62 fold increase during starvation \\
\hline Shc2 & 1.55 & -0.22 & $2.26 \mathrm{E}-06$ & 7.09E-05 & 2.92 fold increase during starvation \\
\hline Thy1 & 1.33 & -0.54 & $2.80 \mathrm{E}-06$ & $8.60 \mathrm{E}-05$ & 2.52 fold increase during starvation \\
\hline Phlda3 & 1.34 & 0.93 & $2.90 \mathrm{E}-06$ & 8.83E-05 & 2.54 fold increase during starvation \\
\hline Cxcl11 & -1.37 & -0.64 & $3.13 \mathrm{E}-06$ & 9.49E-05 & 2.59 fold increase during refeeding \\
\hline Esco2 & 1.43 & 0.02 & $3.18 \mathrm{E}-06$ & 9.61E-05 & 2.70 fold increase during starvation \\
\hline Greb1 & -1.37 & -0.81 & $3.25 \mathrm{E}-06$ & $9.80 \mathrm{E}-05$ & 2.59 fold increase during refeeding \\
\hline Galnt3 & 1.35 & -0.39 & $7.58 \mathrm{E}-06$ & 0.0002098 & 2.56 fold increase during starvation \\
\hline Serpina3h & 1.39 & -0.81 & $8.58 \mathrm{E}-06$ & 0.0002329 & 2.63 fold increase during starvation \\
\hline Lrrc15 & -1.42 & -0.70 & $1.00 \mathrm{E}-05$ & 0.0002683 & 2.67 fold increase during refeeding \\
\hline 4933406I18Rik & -1.45 & -1.40 & $1.65 \mathrm{E}-05$ & 0.0004127 & 2.73 fold increase during refeeding \\
\hline Ccr7 & 1.45 & -1.21 & 1.67E-05 & 0.0004177 & 2.74 fold increase during starvation \\
\hline Matk & 1.38 & -0.68 & $2.39 \mathrm{E}-05$ & 0.0005674 & 2.60 fold increase during starvation \\
\hline Ace2 & 1.49 & -1.22 & 2.44E-05 & 0.000577 & 2.81 fold increase during starvation \\
\hline Slit1 & 1.35 & -1.20 & $2.44 \mathrm{E}-05$ & 0.000577 & 2.55 fold increase during starvation \\
\hline Cbr3 & 1.34 & -0.38 & $2.59 \mathrm{E}-05$ & 0.0006103 & 2.52 fold increase during starvation \\
\hline Sdr16c5 & -1.36 & -0.60 & 2.93E-05 & 0.0006774 & 2.57 fold increase during refeeding \\
\hline Mcoln2 & 1.51 & -1.26 & $2.98 \mathrm{E}-05$ & 0.0006856 & 2.86 fold increase during starvation \\
\hline Sdk1 & 1.45 & -0.80 & 3.43E-05 & 0.0007778 & 2.72 fold increase during starvation \\
\hline Fam40b & 1.36 & -0.91 & $3.65 E-05$ & 0.0008238 & 2.57 fold increase during starvation \\
\hline Gipc2 & 1.56 & -1.34 & 3.70E-05 & 0.0008338 & 2.95 fold increase during starvation \\
\hline Pappa & 1.35 & -0.52 & $6.78 \mathrm{E}-05$ & 0.0013936 & 2.55 fold increase during starvation \\
\hline Myo7b & 1.39 & -1.00 & 7.34E-05 & 0.001497 & 2.62 fold increase during starvation \\
\hline Arl11 & 1.47 & -1.01 & 9.95E-05 & 0.0019429 & 2.77 fold increase during starvation \\
\hline Ccdc46 & 1.40 & -1.32 & 0.000134 & 0.0024903 & 2.64 fold increase during starvation \\
\hline Slc27a6 & 1.33 & -1.37 & 0.0001412 & 0.0025888 & 2.51 fold increase during starvation \\
\hline
\end{tabular}




\section{Table 6: Overall Expression Changes}

\section{b. Listed alphabetically}

\begin{tabular}{|c|c|c|c|c|c|c|}
\hline Gene Name & $\log \mathrm{FC}$ & $\log \mathrm{CPM}$ & $p$ value & FDR & \multicolumn{2}{|r|}{ Absolute Fold Change } \\
\hline 0610007P14Rik & -1.51 & 5.33 & $3.28 \mathrm{E}-11$ & $2.36 \mathrm{E}-09$ & 2.85 & fold increase during refeeding \\
\hline 1600016N20Rik & -1.85 & 0.68 & $4.70 \mathrm{E}-13$ & $4.07 \mathrm{E}-11$ & 3.60 & fold increase during refeeding \\
\hline 3930402G23Rik & 2.21 & -1.07 & $1.15 \mathrm{E}-10$ & $7.66 \mathrm{E}-09$ & 4.64 & fold increase during starvation \\
\hline 4930452B06Rik & -1.45 & 2.22 & $8.87 \mathrm{E}-11$ & $6.03 \mathrm{E}-09$ & 2.74 & fold increase during refeeding \\
\hline 4933406l18Rik & -1.45 & -1.40 & $1.65 \mathrm{E}-05$ & 0.0004127 & 2.73 & fold increase during refeeding \\
\hline 9330182L06Rik & -1.82 & -0.47 & 1.37E-09 & 7.76E-08 & 3.54 & fold increase during refeeding \\
\hline 9530026P05Rik & 1.46 & 0.91 & $1.52 \mathrm{E}-08$ & $7.50 \mathrm{E}-07$ & 2.76 & fold increase during starvation \\
\hline E16Rik & -1.74 & -0.48 & $9.10 \mathrm{E}-10$ & $5.27 \mathrm{E}-08$ & 3.35 & fold increase during $r$ \\
\hline A4gnt & -10.36 & -0.32 & $8.03 \mathrm{E}-48$ & $4.58 \mathrm{E}-45$ & 1313.34 & fold increase during refeeding \\
\hline Aacs & -2.58 & 7.51 & $7.22 \mathrm{E}-30$ & $1.74 \mathrm{E}-27$ & 5.98 & fold increase during refeeding \\
\hline Acaa1b & 1.75 & 9.03 & $1.67 \mathrm{E}-15$ & $1.96 \mathrm{E}-13$ & 3.36 & fold increase during starvation \\
\hline Acaca & -3.02 & 9.58 & $5.18 \mathrm{E}-40$ & $2.15 \mathrm{E}-37$ & 8.10 & fold increase during refeeding \\
\hline Acacb & -3.12 & 8.29 & $2.18 \mathrm{E}-39$ & $8.25 \mathrm{E}-37$ & 8.70 & fold increase during refeeding \\
\hline Acat2 & -2.32 & 6.66 & $5.83 \mathrm{E}-24$ & $1.15 \mathrm{E}-21$ & 5.00 & fold increase during refeeding \\
\hline Ace2 & 1.49 & -1.22 & $2.44 \mathrm{E}-05$ & 0.000577 & 2.81 & fold increase during starvation \\
\hline Acly & -3.35 & 9.76 & $3.08 \mathrm{E}-46$ & $1.46 \mathrm{E}-43$ & 10.20 & fold increase during refeeding \\
\hline Acmsd & -1.66 & 5.50 & $2.40 \mathrm{E}-15$ & $2.70 \mathrm{E}-13$ & 3.16 & fold increase during refeeding \\
\hline Acot1 & 3.42 & 5.04 & $1.35 \mathrm{E}-48$ & $8.17 \mathrm{E}-46$ & 10.72 & fold increase during starvation \\
\hline Acot11 & -1.50 & 3.66 & 3.8 & 2.36E-08 & 2.83 & ng refeeding \\
\hline $\mathrm{Ac}$ & 2.54 & 2.80 & 8.45 & $1.65 \mathrm{E}-21$ & 5.80 & ng starvation \\
\hline Acot3 & 3.31 & 3.40 & $1.01 \mathrm{E}-42$ & $4.48 \mathrm{E}-40$ & 9.90 & vation \\
\hline Acpp & -1.44 & 3.19 & 2.39E-09 & $1.31 \mathrm{E}-07$ & 2.71 & ing refeeding \\
\hline Acsl3 & -1.46 & 5.00 & 8.9 & 6.05E-09 & 2.75 & se during refeeding \\
\hline Acsl5 & -1.39 & 9.12 & $1.52 \mathrm{E}-10$ & $9.94 \mathrm{E}-09$ & 2.63 & fold increase during refeeding \\
\hline Acss2 & -3.44 & 9.13 & $1.66 \mathrm{E}-46$ & $8.17 \mathrm{E}-44$ & 10.84 & fold increase during refeeding \\
\hline Adam11 & 3.32 & 1.30 & $9.82 \mathrm{E}-32$ & $2.61 \mathrm{E}-29$ & 10.00 & fold increase during starvation \\
\hline Adam8 & 1.60 & -0.07 & $4.75 \mathrm{E}-07$ & $1.75 \mathrm{E}-05$ & 3.03 & fold increase during starvation \\
\hline Adamts12 & 1.60 & 0.95 & $1.24 \mathrm{E}-08$ & $6.20 \mathrm{E}-07$ & 3.02 & fold increase during starvation \\
\hline Adamts15 & 1.46 & 1.64 & $6.92 \mathrm{E}-10$ & $4.08 \mathrm{E}-08$ & 2.75 & fold increase during starvation \\
\hline Adcy10 & -3.23 & -1 . & $1.43 \mathrm{E}-18$ & $2.02 \mathrm{E}-16$ & 9.39 & fold increase \\
\hline id & & 7.10 & & $3.33 \mathrm{E}-10$ & 2.79 & vation \\
\hline Afp & -1.55 & 1.26 & 4.1 & $2.94 \mathrm{E}-09$ & 2.94 & during refeeding \\
\hline Agpat9 & 2.18 & 3.32 & -20 & $4.57 \mathrm{E}-18$ & 4.53 & during starvation \\
\hline Akr1b7 & 2.5 & 0.16 & 6.95 & $7.37 \mathrm{E}-13$ & 5.69 & ring starvation \\
\hline Akr1c18 & -1.69 & -0.11 & $4.91 \mathrm{E}-10$ & $2.95 \mathrm{E}-08$ & 3.23 & fold increase during refeeding \\
\hline Aldh112 & -1.54 & 0.18 & $2.30 \mathrm{E}-09$ & $1.27 \mathrm{E}-07$ & 2.91 & fold increase during refeeding \\
\hline Aldob & -1.37 & 13.03 & $7.21 \mathrm{E}-10$ & $4.23 \mathrm{E}-08$ & 2.58 & fold increase during refeeding \\
\hline Aldoc & -1.70 & 3.93 & $1.02 \mathrm{E}-13$ & $9.50 \mathrm{E}-12$ & 3.25 & fold increase during refeeding \\
\hline Amz1 & 1.41 & 0.22 & $6.50 \mathrm{E}-07$ & $2.32 \mathrm{E}-05$ & 2.66 & fold increase during starvation \\
\hline Aqp7 & 2.72 & -1.19 & $4.92 \mathrm{E}-11$ & $3.45 \mathrm{E}-09$ & 6.60 & fold increase during starvation \\
\hline Aqp8 & -2.70 & 5.62 & $1.80 \mathrm{E}-32$ & $4.99 \mathrm{E}-30$ & 6.51 & fold increase during refeeding \\
\hline Areg & 4.31 & -1.13 & $6.85 \mathrm{E}-21$ & $1.12 \mathrm{E}-18$ & 19.85 & fold increase during starvation \\
\hline Arhgap22 & 1.68 & -0.45 & $2.38 \mathrm{E}-07$ & $9.38 \mathrm{E}-06$ & 3.21 & fold increase during starvation \\
\hline Arl11 & 1.47 & -1.01 & $9.95 \mathrm{E}-05$ & 0.0019429 & 2.77 & fold increase during starvation \\
\hline Arl5c & -1.93 & 1.44 & $5.16 \mathrm{E}-16$ & $6.23 \mathrm{E}-14$ & 3.81 & fold increase during refeeding \\
\hline Arntl & 1.72 & 2.62 & $3.26 \mathrm{E}-15$ & $3.57 \mathrm{E}-13$ & 3.31 & fold increase during starvatio \\
\hline
\end{tabular}




\section{Table 6: Overall Expression Changes (continued)}

\section{b. Listed alphabetically}

\begin{tabular}{|c|c|c|c|c|c|c|}
\hline Gene Name & $\log F C$ & $\log C P M$ & $p$ value & FDR & \multicolumn{2}{|c|}{ Absolute Fold Change } \\
\hline Arrdc3 & -1.42 & 7.23 & $1.76 \mathrm{E}-11$ & 1.29E-09 & 2.67 & fold increase during refeeding \\
\hline Atp2b2 & -1.73 & 1.66 & $2.08 \mathrm{E}-14$ & $2.09 \mathrm{E}-12$ & 3.31 & fold increase during refeeding \\
\hline B4galnt3 & -1.40 & 0.69 & $1.62 \mathrm{E}-07$ & $6.61 \mathrm{E}-06$ & 2.63 & fold increase during refeeding \\
\hline BC057022 & 1.40 & 0.24 & 4.00E-08 & 1.83E-06 & 2.64 & fold increase during starvation \\
\hline $\mathrm{Bcl} 2 \mathrm{a} 1 \mathrm{~b}$ & 1.56 & 0.14 & 9.09E-08 & 3.88E-06 & 2.95 & fold increase during starvation \\
\hline Bmper & 1.51 & -0.01 & 1.17E-06 & 3.96E-05 & 2.85 & fold increase during starvation \\
\hline Btla & 1.33 & 0.82 & $6.43 E-07$ & $2.30 \mathrm{E}-05$ & 2.52 & fold increase during starvation \\
\hline C330021F23Rik & -1.98 & 0.14 & $3.63 E-13$ & $3.28 \mathrm{E}-11$ & 3.94 & fold increase during refeeding \\
\hline Cabp2 & -4.41 & -0.29 & $3.61 \mathrm{E}-32$ & 9.77E-30 & 21.29 & fold increase during refeeding \\
\hline Camk1d & -1.92 & 6.03 & 5.35E-18 & $7.32 \mathrm{E}-16$ & 3.79 & fold increase during refeeding \\
\hline Cbr3 & 1.34 & -0.38 & 2.59E-05 & 0.0006103 & 2.52 & fold increase during starvation \\
\hline Ccdc120 & 1.96 & 0.47 & $1.42 \mathrm{E}-11$ & 1.06E-09 & 3.89 & fold increase during starvation \\
\hline Ccdc46 & 1.40 & -1.32 & 0.000134 & 0.0024903 & 2.64 & fold increase during starvation \\
\hline Ccdc69 & -2.24 & -0.25 & 3.33E-14 & $3.16 \mathrm{E}-12$ & 4.73 & fold increase during refeeding \\
\hline Ccr7 & 1.45 & -1.21 & 1.67E-05 & 0.0004177 & 2.74 & fold increase during starvation \\
\hline Cd19 & 1.86 & -1.40 & $2.13 E-06$ & 6.72E-05 & 3.62 & fold increase during starvation \\
\hline Cd209f & -2.77 & -0.25 & $6.83 E-19$ & 9.97E-17 & 6.81 & fold increase during refeeding \\
\hline Cd300lb & 1.80 & 0.68 & $1.44 \mathrm{E}-10$ & 9.52E-09 & 3.48 & fold increase during starvation \\
\hline Cd300If & 1.77 & 0.41 & $4.48 \mathrm{E}-11$ & 3.18E-09 & 3.42 & fold increase during starvation \\
\hline Cd63 & 1.64 & 1.73 & 1.47E-09 & $8.30 \mathrm{E}-08$ & 3.13 & fold increase during starvation \\
\hline Cd83 & 2.00 & 1.06 & $4.31 \mathrm{E}-13$ & $3.81 \mathrm{E}-11$ & 4.00 & fold increase during starvation \\
\hline Cdkn1a & 2.77 & 3.40 & $1.65 \mathrm{E}-30$ & $4.12 \mathrm{E}-28$ & 6.81 & fold increase during starvation \\
\hline Cerkl & 2.24 & 0.02 & 9.90E-16 & $1.18 \mathrm{E}-13$ & 4.73 & fold increase during starvation \\
\hline Ces2b & -3.00 & 0.75 & $7.59 E-28$ & 1.68E-25 & 8.02 & fold increase during refeeding \\
\hline Chrna4 & -6.24 & 3.92 & 4.74E-103 & 3.14E-99 & 75.56 & fold increase during refeeding \\
\hline Cidec & 2.10 & 2.84 & $6.47 \mathrm{E}-18$ & $8.76 \mathrm{E}-16$ & 4.30 & fold increase during starvation \\
\hline Cilp & 1.56 & -0.61 & 1.43E-06 & 4.71E-05 & 2.94 & fold increase during starvation \\
\hline Clec2h & 1.36 & 2.70 & 1.22E-09 & $7.00 \mathrm{E}-08$ & 2.57 & fold increase during starvation \\
\hline Clec7a & 2.00 & 4.14 & $5.34 \mathrm{E}-15$ & $5.67 \mathrm{E}-13$ & 4.00 & fold increase during starvation \\
\hline Col15a1 & -1.32 & 2.45 & 2.22E-09 & 1.23E-07 & 2.50 & fold increase during refeeding \\
\hline Cux2 & 1.75 & -0.22 & $2.10 \mathrm{E}-10$ & 1.34E-08 & 3.37 & fold increase during starvation \\
\hline Cxcl11 & -1.37 & -0.64 & 3.13E-06 & 9.49E-05 & 2.59 & fold increase during refeeding \\
\hline Cxcr4 & 1.71 & 1.14 & $5.67 \mathrm{E}-10$ & 3.39E-08 & 3.28 & fold increase during starvation \\
\hline Cyb5r3 & -1.33 & 8.86 & 2.14E-09 & $1.20 \mathrm{E}-07$ & 2.51 & fold increase during refeeding \\
\hline Cyp1b1 & 1.70 & 0.99 & $1.77 \mathrm{E}-10$ & 1.15E-08 & 3.24 & fold increase during starvation \\
\hline Сyp2a22 & 1.78 & 2.79 & 9.35E-13 & $7.75 \mathrm{E}-11$ & 3.45 & fold increase during starvation \\
\hline Сур2a4 & 3.38 & 5.30 & $3.20 \mathrm{E}-40$ & 1.37E-37 & 10.41 & fold increase during starvation \\
\hline Cyp2b10 & 2.71 & 2.60 & $5.44 \mathrm{E}-27$ & 1.13E-24 & 6.55 & fold increase during starvation \\
\hline Сyp2b9 & 4.01 & 0.99 & $2.28 \mathrm{E}-39$ & 8.39E-37 & 16.13 & fold increase during starvation \\
\hline Cyp3a11 & 1.47 & 10.61 & $3.96 \mathrm{E}-10$ & $2.44 \mathrm{E}-08$ & 2.78 & fold increase during starvation \\
\hline Cyp3a59 & 1.63 & 4.77 & $7.08 \mathrm{E}-12$ & $5.46 \mathrm{E}-10$ & 3.09 & fold increase during starvation \\
\hline Cyp4a10 & 3.99 & 9.34 & 1.69E-65 & $2.80 \mathrm{E}-62$ & 15.89 & fold increase during starvation \\
\hline Cyp4a14 & 4.57 & 9.01 & $7.08 E-82$ & $1.88 \mathrm{E}-78$ & 23.80 & fold increase during starvation \\
\hline Cyp4a31 & 4.36 & 2.92 & 2.99E-59 & $3.06 \mathrm{E}-56$ & 20.59 & fold increase during starvation \\
\hline Cyp4a32 & 2.22 & 4.13 & $2.26 \mathrm{E}-23$ & $4.22 \mathrm{E}-21$ & 4.65 & fold increase during starvation \\
\hline Cyp51 & -3.53 & 7.62 & $8.28 \mathrm{E}-48$ & $4.58 \mathrm{E}-45$ & 11.55 & fold increase during refeeding \\
\hline
\end{tabular}




\section{Table 6: Overall Expression Changes (continued)}

\section{b. Listed alphabetically}

\begin{tabular}{|c|c|c|c|c|c|c|}
\hline Gene Name & $\log F C$ & $\log C P M$ & $p$ value & FDR & \multicolumn{2}{|c|}{ Absolute Fold Change } \\
\hline D7Ertd143e & 2.04 & 3.15 & 2.89E-21 & 4.92E-19 & 4.12 & fold increase during starvation \\
\hline Dak & -1.59 & 7.84 & $2.20 \mathrm{E}-12$ & $1.77 \mathrm{E}-10$ & 3.00 & fold increase during refeeding \\
\hline Dbi & -1.44 & 8.59 & $1.41 \mathrm{E}-11$ & 1.06E-09 & 2.71 & fold increase during refeeding \\
\hline Dhcr7 & -2.52 & 5.10 & $6.62 \mathrm{E}-28$ & $1.49 \mathrm{E}-25$ & 5.74 & fold increase during refeeding \\
\hline Dkk3 & 1.42 & 1.86 & 6.33E-08 & $2.77 E-06$ & 2.68 & fold increase during starvation \\
\hline Dnahc17 & -1.41 & -0.08 & $4.40 \mathrm{E}-07$ & 1.64E-05 & 2.65 & fold increase during refeeding \\
\hline Dntt & -2.69 & 2.44 & 1.54E-29 & $3.60 \mathrm{E}-27$ & 6.47 & fold increase during refeeding \\
\hline E2f2 & -1.35 & 0.23 & 1.50E-08 & $7.46 \mathrm{E}-07$ & 2.54 & fold increase during refeeding \\
\hline Echdc1 & -1.54 & 4.43 & $5.96 \mathrm{E}-12$ & $4.62 \mathrm{E}-10$ & 2.92 & fold increase during refeeding \\
\hline Eci2 & 1.36 & 7.83 & $2.60 \mathrm{E}-10$ & 1.63E-08 & 2.56 & fold increase during starvation \\
\hline Eda2r & 2.57 & 1.46 & $2.64 \mathrm{E}-20$ & $4.27 \mathrm{E}-18$ & 5.92 & fold increase during starvation \\
\hline Egr1 & 2.25 & 2.05 & $2.52 \mathrm{E}-17$ & $3.31 \mathrm{E}-15$ & 4.76 & fold increase during starvation \\
\hline Ehhadh & 1.78 & 8.48 & $1.78 \mathrm{E}-15$ & $2.08 \mathrm{E}-13$ & 3.44 & fold increase during starvation \\
\hline Eln & 1.95 & 1.67 & $1.45 \mathrm{E}-13$ & $1.33 \mathrm{E}-11$ & 3.87 & fold increase during starvation \\
\hline Elovl3 & 1.81 & 6.27 & $2.14 \mathrm{E}-19$ & $3.30 \mathrm{E}-17$ & 3.52 & fold increase during starvation \\
\hline Elovi6 & -3.93 & 9.52 & 5.99E-60 & $6.63 E-57$ & 15.23 & fold increase during refeeding \\
\hline Elovl7 & 1.43 & 0.49 & $1.86 \mathrm{E}-06$ & 5.93E-05 & 2.70 & fold increase during starvation \\
\hline Esco2 & 1.43 & 0.02 & $3.18 E-06$ & 9.61E-05 & 2.70 & fold increase during starvation \\
\hline Fabp5 & -3.52 & 8.05 & 3.69E-51 & $2.58 \mathrm{E}-48$ & 11.45 & fold increase during refeeding \\
\hline Fabp7 & 1.92 & 1.00 & $5.33 E-13$ & 4.57E-11 & 3.80 & fold increase during starvation \\
\hline Fads1 & -1.35 & 9.19 & $4.49 \mathrm{E}-10$ & $2.72 \mathrm{E}-08$ & 2.55 & fold increase during refeeding \\
\hline Fads3 & -1.53 & 4.72 & $1.61 \mathrm{E}-12$ & $1.30 \mathrm{E}-10$ & 2.89 & fold increase during refeeding \\
\hline Fam107a & 1.55 & 3.96 & $1.32 \mathrm{E}-13$ & $1.22 \mathrm{E}-11$ & 2.92 & fold increase during starvation \\
\hline Fam187b & -1.36 & 2.49 & $6.11 \mathrm{E}-10$ & $3.62 \mathrm{E}-08$ & 2.56 & fold increase during refeeding \\
\hline Fam198a & -2.16 & 2.46 & 4.99E-20 & $7.88 \mathrm{E}-18$ & 4.48 & fold increase during refeeding \\
\hline Fam40b & 1.36 & -0.91 & 3.65E-05 & 0.0008238 & 2.57 & fold increase during starvation \\
\hline Fam81a & 2.06 & 0.79 & $5.84 \mathrm{E}-14$ & $5.50 \mathrm{E}-12$ & 4.17 & fold increase during starvation \\
\hline Fam89a & 1.86 & 0.39 & $9.90 \mathrm{E}-13$ & $8.11 \mathrm{E}-11$ & 3.63 & fold increase during starvation \\
\hline Fasn & -4.80 & 11.39 & 9.72E-81 & $2.15 \mathrm{E}-77$ & 27.78 & fold increase during refeeding \\
\hline $\mathrm{Fbx032}$ & 1.78 & 2.17 & $1.77 \mathrm{E}-13$ & $1.62 \mathrm{E}-11$ & 3.43 & fold increase during starvation \\
\hline Fdft1 & -1.85 & 6.88 & $3.56 \mathrm{E}-16$ & $4.42 \mathrm{E}-14$ & 3.59 & fold increase during refeeding \\
\hline Fdps & -4.62 & 8.71 & $1.58 \mathrm{E}-75$ & 3.00E-72 & 24.67 & fold increase during refeeding \\
\hline Fgf21 & 2.41 & 1.99 & $4.30 \mathrm{E}-19$ & $6.48 \mathrm{E}-17$ & 5.32 & fold increase during starvation \\
\hline Fkbp11 & -1.40 & 3.85 & $5.34 \mathrm{E}-11$ & 3.69E-09 & 2.63 & fold increase during refeeding \\
\hline Fmo3 & 2.23 & 2.26 & $2.85 E-22$ & $4.98 \mathrm{E}-20$ & 4.69 & fold increase during starvation \\
\hline Fmr1nb & -5.05 & -0.15 & $2.13 E-39$ & $8.25 E-37$ & 33.12 & fold increase during refeeding \\
\hline Fras1 & -1.63 & 3.75 & $7.68 \mathrm{E}-13$ & $6.41 \mathrm{E}-11$ & 3.09 & fold increase during refeeding \\
\hline Frat1 & 1.33 & 2.44 & 1.28E-09 & $7.28 \mathrm{E}-08$ & 2.52 & fold increase during starvation \\
\hline Frat2 & 1.41 & 0.59 & 4.53E-08 & 2.04E-06 & 2.65 & fold increase during starvation \\
\hline G6pdx & -2.52 & 4.89 & $2.15 E-29$ & 4.93E-27 & 5.72 & fold increase during refeeding \\
\hline Gabbr2 & -1.67 & 4.59 & $3.13 E-14$ & $3.01 \mathrm{E}-12$ & 3.18 & fold increase during refeeding \\
\hline Gadd45g & 1.59 & 4.95 & $1.00 \mathrm{E}-14$ & $1.03 E-12$ & 3.00 & fold increase during starvation \\
\hline Gale & -3.10 & 4.08 & $9.77 \mathrm{E}-40$ & 3.93E-37 & 8.58 & fold increase during refeeding \\
\hline Galnt3 & 1.35 & -0.39 & $7.58 \mathrm{E}-06$ & 0.0002098 & 2.56 & fold increase during starvation \\
\hline Gbe1 & -1.36 & 7.94 & $8.85 E-10$ & $5.15 \mathrm{E}-08$ & 2.57 & fold increase during refeeding \\
\hline Gck & -1.85 & 8.08 & $5.98 \mathrm{E}-17$ & $7.56 \mathrm{E}-15$ & 3.62 & fold increase during refeeding \\
\hline
\end{tabular}




\section{Table 6: Overall Expression Changes (continued)}

\section{b. Listed alphabetically}

\begin{tabular}{|c|c|c|c|c|c|c|}
\hline Gene Name & $\log F C$ & $\log C P M$ & $p$ value & FDR & \multicolumn{2}{|c|}{ Absolute Fold Change } \\
\hline Gdf15 & 1.76 & 1.33 & $7.38 \mathrm{E}-10$ & 4.32E-08 & 3.38 & fold increase during starvation \\
\hline Gdpd1 & 1.49 & 0.82 & 3.86E-08 & 1.79E-06 & 2.82 & fold increase during starvation \\
\hline Gipc2 & 1.56 & -1.34 & 3.70E-05 & 0.0008338 & 2.95 & fold increase during starvation \\
\hline Gldn & -1.39 & -0.24 & 1.89E-07 & 7.58E-06 & 2.62 & fold increase during refeeding \\
\hline Gm4952 & 2.43 & 5.77 & 8.93E-28 & $1.94 \mathrm{E}-25$ & 5.38 & fold increase during starvation \\
\hline Gm6484 & -3.54 & 6.14 & 3.05E-55 & $2.53 \mathrm{E}-52$ & 11.64 & fold increase during refeeding \\
\hline Gm6614 & 1.75 & 0.38 & $2.60 \mathrm{E}-10$ & 1.63E-08 & 3.35 & fold increase during starvation \\
\hline Gm6907 & 1.40 & 1.06 & 9.78E-09 & 4.97E-07 & 2.64 & fold increase during starvation \\
\hline Gnat1 & -1.35 & 2.35 & 1.27E-09 & 7.27E-08 & 2.56 & fold increase during refeeding \\
\hline Gnat2 & -1.71 & 0.33 & $7.56 \mathrm{E}-12$ & 5.77E-10 & 3.28 & fold increase during refeeding \\
\hline Gpam & -1.92 & 8.68 & $5.07 \mathrm{E}-18$ & $7.01 \mathrm{E}-16$ & 3.78 & fold increase during refeeding \\
\hline Gpi1 & -1.73 & 7.86 & $2.92 \mathrm{E}-15$ & $3.23 \mathrm{E}-13$ & 3.32 & fold increase during refeeding \\
\hline Gpnmb & 4.61 & 3.32 & 2.93E-62 & 3.89E-59 & 24.41 & fold increase during starvation \\
\hline Gpr110 & -1.45 & 4.25 & $2.06 \mathrm{E}-10$ & 1.32E-08 & 2.73 & fold increase during refeeding \\
\hline Gpr35 & 1.45 & 0.74 & 3.52E-08 & 1.64E-06 & 2.74 & fold increase during starvation \\
\hline Greb1 & -1.37 & -0.81 & $3.25 E-06$ & 9.80E-05 & 2.59 & fold increase during refeeding \\
\hline Gstm2 & -1.79 & 5.61 & $2.92 \mathrm{E}-17$ & $3.80 \mathrm{E}-15$ & 3.47 & fold increase during refeeding \\
\hline Gstm6 & -1.42 & 6.18 & $1.09 \mathrm{E}-10$ & 7.33E-09 & 2.68 & fold increase during refeeding \\
\hline Gucy2c & 2.35 & 0.02 & $2.70 \mathrm{E}-14$ & $2.63 \mathrm{E}-12$ & 5.08 & fold increase during starvation \\
\hline $\mathrm{H} 19$ & 6.16 & 2.87 & $8.31 \mathrm{E}-85$ & $2.76 \mathrm{E}-81$ & 71.28 & fold increase during starvation \\
\hline $\mathrm{H} 2-\mathrm{BI}$ & -1.44 & -0.39 & $5.98 \mathrm{E}-07$ & $2.16 \mathrm{E}-05$ & 2.71 & fold increase during refeeding \\
\hline Hapln1 & -2.54 & 0.11 & $1.22 \mathrm{E}-19$ & $1.90 \mathrm{E}-17$ & 5.80 & fold increase during refeeding \\
\hline Hapln4 & -1.83 & 1.43 & $2.15 \mathrm{E}-14$ & $2.15 \mathrm{E}-12$ & 3.55 & fold increase during refeeding \\
\hline Haus7 & -1.40 & 3.42 & $2.06 \mathrm{E}-10$ & 1.32E-08 & 2.64 & fold increase during refeeding \\
\hline Hen3 & -3.01 & 4.67 & $2.60 \mathrm{E}-36$ & 8.02E-34 & 8.08 & fold increase during refeeding \\
\hline Hes6 & -1.71 & 6.40 & $3.02 \mathrm{E}-14$ & $2.92 \mathrm{E}-12$ & 3.28 & fold increase during refeeding \\
\hline $\mathrm{Hmgcr}$ & -2.91 & 8.18 & $2.19 \mathrm{E}-35$ & $6.61 \mathrm{E}-33$ & 7.54 & fold increase during refeeding \\
\hline Hsbp1I1 & -1.76 & 1.48 & $2.75 \mathrm{E}-13$ & $2.50 \mathrm{E}-11$ & 3.39 & fold increase during refeeding \\
\hline Hsd17b6 & 2.00 & 6.49 & $1.38 \mathrm{E}-17$ & $1.84 \mathrm{E}-15$ & 4.01 & fold increase during starvation \\
\hline Hsd17b7 & -1.50 & 6.45 & $1.26 \mathrm{E}-10$ & 8.35E-09 & 2.82 & fold increase during refeeding \\
\hline Hspa1a & -1.45 & 2.07 & $4.54 \mathrm{E}-10$ & $2.74 \mathrm{E}-08$ & 2.74 & fold increase during refeeding \\
\hline Htra3 & 1.60 & 1.35 & 2.70E-09 & 1.47E-07 & 3.03 & fold increase during starvation \\
\hline Icosl & 1.32 & 0.85 & 4.73E-07 & 1.75E-05 & 2.50 & fold increase during starvation \\
\hline Idi1 & -3.54 & 7.62 & $1.05 E-47$ & $5.58 \mathrm{E}-45$ & 11.66 & fold increase during refeeding \\
\hline $\operatorname{lgfbp1}$ & 1.65 & 6.77 & $4.45 \mathrm{E}-15$ & $4.76 \mathrm{E}-13$ & 3.14 & fold increase during starvation \\
\hline Ihh & -1.42 & 1.51 & $4.43 E-10$ & $2.70 \mathrm{E}-08$ & 2.68 & fold increase during refeeding \\
\hline II1rn & 1.60 & 0.91 & 1.82E-08 & 8.86E-07 & 3.03 & fold increase during starvation \\
\hline$\| 7 \mathrm{r}$ & 1.56 & -0.15 & $2.24 \mathrm{E}-07$ & 8.86E-06 & 2.95 & fold increase during starvation \\
\hline Insig1 & -2.52 & 8.62 & $4.70 \mathrm{E}-27$ & 9.91E-25 & 5.72 & fold increase during refeeding \\
\hline Insig2 & 1.65 & 7.25 & $1.88 \mathrm{E}-15$ & $2.15 \mathrm{E}-13$ & 3.14 & fold increase during starvation \\
\hline Islr & 1.53 & 1.74 & 2.22E-09 & 1.23E-07 & 2.88 & fold increase during starvation \\
\hline Itgax & 1.48 & 1.85 & 4.17E-08 & 1.90E-06 & 2.80 & fold increase during starvation \\
\hline Khk & -1.80 & 8.60 & $1.81 \mathrm{E}-15$ & $2.09 \mathrm{E}-13$ & 3.48 & fold increase during refeeding \\
\hline Klk1b4 & -1.48 & 1.61 & $1.51 \mathrm{E}-10$ & 9.92E-09 & 2.79 & fold increase during refeeding \\
\hline Krt23 & 3.18 & 1.77 & $1.27 \mathrm{E}-31$ & $3.30 \mathrm{E}-29$ & 9.07 & fold increase during starvation \\
\hline Lamb3 & 1.42 & 2.24 & $8.85 E-11$ & 6.03E-09 & 2.68 & fold increase during starvation \\
\hline
\end{tabular}




\section{Table 6: Overall Expression Changes (continued)}

\section{b. Listed alphabetically}

\begin{tabular}{|c|c|c|c|c|c|c|}
\hline Gene Name & $\log F C$ & $\log C P M$ & $p$ value & FDR & \multicolumn{2}{|c|}{ Absolute Fold Change } \\
\hline Lbp & 1.70 & 5.65 & $3.79 E-15$ & $4.12 \mathrm{E}-13$ & 3.25 & fold increase during starvation \\
\hline Ldlr & -1.35 & 7.39 & 3.05E-09 & $1.65 \mathrm{E}-07$ & 2.55 & fold increase during refeeding \\
\hline Lgals4 & 1.98 & 2.91 & $2.24 \mathrm{E}-17$ & $2.97 \mathrm{E}-15$ & 3.94 & fold increase during starvation \\
\hline Lhx6 & -1.39 & 2.05 & 3.17E-09 & 1.70E-07 & 2.62 & fold increase during refeeding \\
\hline Lpl & 1.66 & 4.64 & $4.54 \mathrm{E}-11$ & 3.20E-09 & 3.16 & fold increase during starvation \\
\hline Lrg1 & 1.51 & 7.28 & $6.78 \mathrm{E}-13$ & $5.73 E-11$ & 2.84 & fold increase during starvation \\
\hline Lrrc15 & -1.42 & -0.70 & $1.00 \mathrm{E}-05$ & 0.0002683 & 2.67 & fold increase during refeeding \\
\hline Lss & -2.88 & 6.36 & $1.14 \mathrm{E}-34$ & $3.30 \mathrm{E}-32$ & 7.36 & fold increase during refeeding \\
\hline Ly6d & 3.16 & 1.11 & $7.44 \mathrm{E}-27$ & $1.52 \mathrm{E}-24$ & 8.94 & fold increase during starvation \\
\hline Magi2 & 1.85 & -1.41 & $1.70 \mathrm{E}-06$ & $5.46 \mathrm{E}-05$ & 3.60 & fold increase during starvation \\
\hline Matk & 1.38 & -0.68 & 2.39E-05 & 0.0005674 & 2.60 & fold increase during starvation \\
\hline Mcoln2 & 1.51 & -1.26 & 2.98E-05 & 0.0006856 & 2.86 & fold increase during starvation \\
\hline Me1 & -3.26 & 8.30 & $1.88 \mathrm{E}-43$ & $8.62 \mathrm{E}-41$ & 9.59 & fold increase during refeeding \\
\hline Mfsd7c & 2.34 & 2.19 & $6.57 \mathrm{E}-21$ & 1.09E-18 & 5.07 & fold increase during starvation \\
\hline Mid1ip1 & -2.92 & 5.72 & $6.49 E-38$ & $2.15 \mathrm{E}-35$ & 7.57 & fold increase during refeeding \\
\hline Mmp12 & 2.26 & 2.70 & $3.91 \mathrm{E}-17$ & $5.04 \mathrm{E}-15$ & 4.80 & fold increase during starvation \\
\hline Mmp2 & 1.81 & 3.01 & $5.58 \mathrm{E}-13$ & $4.75 \mathrm{E}-11$ & 3.52 & fold increase during starvation \\
\hline Mmp27 & 1.58 & 0.67 & $3.15 \mathrm{E}-09$ & $1.70 \mathrm{E}-07$ & 3.00 & fold increase during starvation \\
\hline Moxd1 & 2.60 & 1.00 & $6.31 \mathrm{E}-22$ & 1.09E-19 & 6.08 & fold increase during starvation \\
\hline Mt1 & 3.08 & 6.33 & $5.39 \mathrm{E}-38$ & $1.84 \mathrm{E}-35$ & 8.47 & fold increase during starvation \\
\hline Mt2 & 3.80 & 5.43 & $1.74 \mathrm{E}-53$ & $1.28 \mathrm{E}-50$ & 13.89 & fold increase during starvation \\
\hline Mvd & -3.99 & 4.25 & $2.87 E-56$ & $2.72 \mathrm{E}-53$ & 15.84 & fold increase during refeeding \\
\hline Mvk & -2.33 & 4.45 & $2.63 E-23$ & 4.84E-21 & 5.03 & fold increase during refeeding \\
\hline Mycn & -1.69 & 1.68 & $7.49 \mathrm{E}-12$ & $5.75 \mathrm{E}-10$ & 3.22 & fold increase during refeeding \\
\hline Myh6 & 3.84 & -0.75 & $2.84 \mathrm{E}-22$ & 4.98E-20 & 14.34 & fold increase during starvation \\
\hline Myo7b & 1.39 & -1.00 & 7.34E-05 & 0.001497 & 2.62 & fold increase during starvation \\
\hline Myom3 & 1.39 & 0.05 & 1.93E-07 & 7.70E-06 & 2.61 & fold increase during starvation \\
\hline N28178 & 2.07 & -0.63 & $1.97 \mathrm{E}-10$ & 1.27E-08 & 4.20 & fold increase during starvation \\
\hline Naip1 & 1.36 & 0.71 & 8.64E-08 & 3.71E-06 & 2.56 & fold increase during starvation \\
\hline Neto2 & 1.41 & 0.85 & 1.66E-07 & $6.72 \mathrm{E}-06$ & 2.66 & fold increase during starvation \\
\hline $\mathrm{Nfe} 2$ & -2.15 & -0.35 & $4.01 \mathrm{E}-13$ & $3.57 \mathrm{E}-11$ & 4.43 & fold increase during refeeding \\
\hline Nlrp12 & 1.86 & 5.94 & 6.39E-19 & $9.52 \mathrm{E}-17$ & 3.63 & fold increase during starvation \\
\hline Nr1i3 & 1.36 & 7.05 & $5.46 \mathrm{E}-11$ & 3.76E-09 & 2.56 & fold increase during starvation \\
\hline Nrg1 & 2.47 & 1.37 & 3.68E-19 & $5.62 \mathrm{E}-17$ & 5.53 & fold increase during starvation \\
\hline Nsdhl & -3.86 & 5.93 & $5.31 \mathrm{E}-56$ & 4.70E-53 & 14.56 & fold increase during refeeding \\
\hline Nupr1 & 4.15 & 2.69 & $4.88 \mathrm{E}-50$ & $3.24 \mathrm{E}-47$ & 17.78 & fold increase during starvation \\
\hline Odf3b & 1.44 & 1.53 & $9.58 \mathrm{E}-10$ & 5.53E-08 & 2.72 & fold increase during starvation \\
\hline Odz4 & 1.50 & -0.23 & $1.55 \mathrm{E}-06$ & 4.99E-05 & 2.83 & fold increase during starvation \\
\hline Osbp2 & 2.46 & -0.86 & $4.28 \mathrm{E}-12$ & $3.34 \mathrm{E}-10$ & 5.51 & fold increase during starvation \\
\hline Osbpl3 & 2.35 & 2.98 & $6.55 \mathrm{E}-21$ & $1.09 \mathrm{E}-18$ & 5.11 & fold increase during starvation \\
\hline Pappa & 1.35 & -0.52 & 6.78E-05 & 0.0013936 & 2.55 & fold increase during starvation \\
\hline Pcsk9 & -3.70 & 5.78 & 1.67E-53 & $1.28 \mathrm{E}-50$ & 12.99 & fold increase during refeeding \\
\hline Pdzk1ip1 & -3.71 & 3.01 & $1.18 \mathrm{E}-48$ & $7.48 \mathrm{E}-46$ & 13.09 & fold increase during refeeding \\
\hline $\mathrm{Pgd}$ & -2.22 & 4.70 & $3.08 \mathrm{E}-23$ & $5.61 \mathrm{E}-21$ & 4.65 & fold increase during refeeding \\
\hline Phlda3 & 1.34 & 0.93 & $2.90 \mathrm{E}-06$ & 8.83E-05 & 2.54 & fold increase during starvation \\
\hline Pitx3 & 3.08 & 0.39 & $2.17 \mathrm{E}-27$ & $4.64 \mathrm{E}-25$ & 8.44 & fold increase during starvation \\
\hline
\end{tabular}




\section{Table 6: Overall Expression Changes (continued)}

\section{b. Listed alphabetically}

\begin{tabular}{|c|c|c|c|c|c|}
\hline Gene Name & $\log F C$ & $\log C P M$ & $p$ value & FDR & Absolute Fold Change \\
\hline Pklr & -2.70 & 8.77 & 9.53E-31 & $2.43 \mathrm{E}-28$ & fold increase during refeeding \\
\hline Pla2g12a & 1.53 & 3.74 & $3.51 \mathrm{E}-11$ & 2.52E-09 & 2.88 fold increase during starvation \\
\hline Pla2g7 & 1.55 & 2.86 & $5.15 \mathrm{E}-11$ & 3.59E-09 & fold increase during starvation \\
\hline Pmm1 & 1.84 & 0.74 & $1.63 \mathrm{E}-11$ & 1.20E-09 & fold increase during starvation \\
\hline Pmvk & -3.03 & 5.71 & $4.08 E-38$ & 1.43E-35 & fold increase during refeeding \\
\hline Pnpla3 & -5.77 & 5.66 & $1.02 E-102$ & 4.53E-99 & fold increase during refeeding \\
\hline Pnpla5 & -9.77 & 2.25 & $7.44 \mathrm{E}-105$ & 9.87E-101 & fold increase during refeeding \\
\hline Por & 1.34 & 9.06 & $2.69 \mathrm{E}-10$ & 1.69E-08 & fold increase during starvation \\
\hline Prokr1 & 2.27 & -1.34 & 4.02E-09 & $2.13 E-07$ & fold increase during starvation \\
\hline Prrg4 & 1.40 & 0.54 & 1.09E-06 & 3.70E-05 & fold increase during starvation \\
\hline Prss8 & -1.75 & 3.06 & $8.62 E-15$ & 8.94E-13 & fold increase during refeeding \\
\hline Psat1 & -1.47 & 2.29 & $4.56 \mathrm{E}-13$ & $3.98 \mathrm{E}-11$ & fold increase during refeeding \\
\hline Psrc1 & 4.09 & -0.78 & $4.22 E-23$ & $7.56 \mathrm{E}-21$ & fold increase during starvation \\
\hline Pstpip2 & -2.84 & 4.09 & 1.36E-33 & 3.84E-31 & fold increase during refeeding \\
\hline Ptpro & 1.50 & -0.13 & $1.21 \mathrm{E}-07$ & 5.03E-06 & fold increase during starvation \\
\hline Rab11fip4 & 1.94 & 1.35 & $1.63 E-14$ & $1.65 \mathrm{E}-12$ & fold increase during starvation \\
\hline Rab30 & 2.03 & 2.88 & $7.40 \mathrm{E}-19$ & 1.07E-16 & fold increase during starvation \\
\hline Rad51I1 & 1.48 & 0.13 & 1.47E-06 & 4.82E-05 & fold increase during starvation \\
\hline Ralgps1 & -1.35 & 2.04 & 3.97E-09 & $2.11 \mathrm{E}-07$ & fold increase during refeeding \\
\hline Rap1gap2 & 1.54 & 1.63 & 2.49E-09 & 1.36E-07 & fold increase during starvation \\
\hline Rasal1 & 2.20 & -0.93 & $4.28 \mathrm{E}-10$ & $2.62 \mathrm{E}-08$ & fold increase during starvation \\
\hline Rdh11 & -3.47 & 5.98 & $2.82 \mathrm{E}-47$ & 1.44E-44 & fold increase during refeeding \\
\hline Rec8 & -1.89 & 0.50 & $9.82 \mathrm{E}-13$ & $8.10 \mathrm{E}-11$ & fold increase during refeeding \\
\hline $\mathrm{Rfx} 4$ & 1.86 & -0.04 & $1.61 \mathrm{E}-12$ & $1.30 \mathrm{E}-10$ & fold increase during starvation \\
\hline Rgs1 & 1.37 & 1.21 & 1.05E-07 & 4.43E-06 & fold increase during starvation \\
\hline Rnd2 & -1.93 & 1.54 & $3.95 E-15$ & $4.26 \mathrm{E}-13$ & fold increase during refeeding \\
\hline Rsad2 & 1.37 & 3.50 & 3.36E-09 & 1.79E-07 & fold increase during starvation \\
\hline Rwdd2a & -2.34 & 1.13 & $6.52 \mathrm{E}-19$ & $9.61 \mathrm{E}-17$ & fold increase during refeeding \\
\hline Sc4mol & -3.02 & 7.54 & $8.92 E-37$ & $2.89 E-34$ & fold increase during refeeding \\
\hline Sc5d & -1.86 & 8.50 & $4.39 \mathrm{E}-17$ & $5.60 \mathrm{E}-15$ & fold increase during refeeding \\
\hline Scd1 & -2.25 & 14.50 & $1.68 \mathrm{E}-23$ & $3.18 \mathrm{E}-21$ & fold increase during refeeding \\
\hline Scube1 & 1.93 & 0.59 & $1.07 \mathrm{E}-11$ & $8.04 \mathrm{E}-10$ & fold increase during starvation \\
\hline Sdk1 & 1.45 & -0.80 & 3.43E-05 & 0.0007778 & fold increase during starvation \\
\hline Sdr16c5 & -1.36 & -0.60 & 2.93E-05 & 0.0006774 & fold increase during refeeding \\
\hline Sec61b & -1.55 & 5.84 & $7.48 \mathrm{E}-13$ & $6.28 \mathrm{E}-11$ & fold increase during refeeding \\
\hline Sec61g & -1.56 & 5.98 & $4.36 \mathrm{E}-13$ & 3.83E-11 & fold increase during refeeding \\
\hline Sell & 1.70 & 0.69 & $8.90 \mathrm{E}-11$ & 6.03E-09 & fold increase during starvation \\
\hline Sema3g & 2.00 & -0.92 & 6.91E-09 & 3.58E-07 & fold increase during starvation \\
\hline SEPT5 & -2.54 & -0.18 & $1.04 \mathrm{E}-18$ & $1.49 \mathrm{E}-16$ & fold increase during refeeding \\
\hline Serpina3h & 1.39 & -0.81 & $8.58 \mathrm{E}-06$ & 0.0002329 & fold increase during starvation \\
\hline Serpina5 & -2.11 & 0.94 & $4.93 E-16$ & $6.00 \mathrm{E}-14$ & fold increase during refeeding \\
\hline Serpinb1a & 1.71 & -0.41 & 1.80E-08 & $8.81 \mathrm{E}-07$ & fold increase during starvation \\
\hline Sgsm1 & -2.84 & 1.90 & 9.76E-30 & $2.31 \mathrm{E}-27$ & fold increase during refeeding \\
\hline Sh3pxd2b & 1.80 & 2.12 & $2.52 \mathrm{E}-12$ & $2.00 \mathrm{E}-10$ & fold increase during starvation \\
\hline Shc2 & 1.55 & -0.22 & $2.26 \mathrm{E}-06$ & 7.09E-05 & fold increase during starvation \\
\hline Slc10a2 & -1.58 & 4.17 & $4.01 \mathrm{E}-13$ & $3.57 \mathrm{E}-11$ & fold increase during refeeding \\
\hline
\end{tabular}




\section{Table 6: Overall Expression Changes (continued)}

\section{b. Listed alphabetically}

\begin{tabular}{|c|c|c|c|c|c|c|}
\hline Gene Name & $\log \mathrm{FC}$ & $\log C P M$ & $p$ value & FDR & \multicolumn{2}{|c|}{ Absolute Fold Change } \\
\hline Slc13a5 & 1.38 & 0.45 & 1.00E-06 & 3.45E-05 & 2.61 & fold increase during starvation \\
\hline Slc17a4 & -1.68 & 5.25 & $7.34 \mathrm{E}-15$ & $7.67 \mathrm{E}-13$ & 3.20 & fold increase during refeeding \\
\hline Slc22a28 & -1.58 & 2.56 & $5.17 \mathrm{E}-11$ & 3.59E-09 & 2.99 & fold increase during refeeding \\
\hline Slc22a5 & 1.65 & 4.81 & $2.64 \mathrm{E}-14$ & $2.61 \mathrm{E}-12$ & 3.13 & fold increase during starvation \\
\hline Slc25a34 & 1.49 & 3.01 & $2.89 \mathrm{E}-11$ & $2.10 \mathrm{E}-09$ & 2.80 & fold increase during starvation \\
\hline Slc27a1 & 1.78 & 2.90 & $2.62 \mathrm{E}-15$ & $2.92 \mathrm{E}-13$ & 3.43 & fold increase during starvation \\
\hline Slc27a2 & 1.46 & 10.55 & $1.05 \mathrm{E}-11$ & $7.95 \mathrm{E}-10$ & 2.74 & fold increase during starvation \\
\hline Slc27a6 & 1.33 & -1.37 & 0.0001412 & 0.0025888 & 2.51 & fold increase during starvation \\
\hline Slc2a4 & -3.14 & 2.03 & $3.92 \mathrm{E}-38$ & $1.40 \mathrm{E}-35$ & 8.82 & fold increase during refeeding \\
\hline Slc41a3 & 1.40 & 2.65 & 1.85E-08 & 8.95E-07 & 2.64 & fold increase during starvation \\
\hline Slc7a4 & -1.62 & 0.90 & $2.19 \mathrm{E}-11$ & 1.60E-09 & 3.07 & fold increase during refeeding \\
\hline Slit1 & 1.35 & -1.20 & $2.44 \mathrm{E}-05$ & 0.000577 & 2.55 & fold increase during starvation \\
\hline Snhg11 & -1.91 & 1.39 & $2.69 \mathrm{E}-14$ & $2.63 \mathrm{E}-12$ & 3.76 & fold increase during refeeding \\
\hline Snurf & -1.53 & -0.83 & 4.73E-07 & $1.75 \mathrm{E}-05$ & 2.88 & fold increase during refeeding \\
\hline Sorcs2 & -2.60 & -0.40 & $3.71 \mathrm{E}-16$ & $4.56 \mathrm{E}-14$ & 6.08 & fold increase during refeeding \\
\hline Spink3 & 1.74 & -0.72 & $1.04 \mathrm{E}-06$ & $3.55 \mathrm{E}-05$ & 3.35 & fold increase during starvation \\
\hline Sqle & -4.20 & 6.54 & $3.48 \mathrm{E}-64$ & $5.13 \mathrm{E}-61$ & 18.35 & fold increase during refeeding \\
\hline Srebf1 & -2.84 & 8.56 & $2.47 \mathrm{E}-36$ & $7.80 \mathrm{E}-34$ & 7.14 & fold increase during refeeding \\
\hline Stard4 & -1.98 & 5.46 & $4.08 \mathrm{E}-18$ & $5.70 \mathrm{E}-16$ & 3.94 & fold increase during refeeding \\
\hline Sucnr1 & -1.60 & 4.44 & $2.32 \mathrm{E}-12$ & $1.86 \mathrm{E}-10$ & 3.03 & fold increase during refeeding \\
\hline Tcp1112 & 1.53 & 4.10 & $5.10 \mathrm{E}-13$ & $4.40 \mathrm{E}-11$ & 2.89 & fold increase during starvation \\
\hline Tctex1d4 & 1.38 & -0.63 & $8.84 \mathrm{E}-07$ & 3.09E-05 & 2.60 & fold increase during starvation \\
\hline Thbs2 & 1.32 & 3.27 & 9.37E-08 & 3.98E-06 & 2.50 & fold increase during starvation \\
\hline Themis & 1.69 & -0.74 & $2.08 \mathrm{E}-08$ & 9.99E-07 & 3.23 & fold increase during starvation \\
\hline Thrsp & -2.70 & 10.44 & $2.00 \mathrm{E}-30$ & 4.91E-28 & 6.48 & fold increase during refeeding \\
\hline Thy1 & 1.33 & -0.54 & $2.80 \mathrm{E}-06$ & $8.60 \mathrm{E}-05$ & 2.52 & fold increase during starvation \\
\hline Tkt & -1.49 & 7.40 & $1.50 \mathrm{E}-11$ & 1.11E-09 & 2.81 & fold increase during refeeding \\
\hline Tnnc1 & -2.22 & -0.16 & $1.62 \mathrm{E}-14$ & $1.65 \mathrm{E}-12$ & 4.67 & fold increase during refeeding \\
\hline Trem2 & 2.31 & 1.01 & $2.03 E-15$ & $2.31 \mathrm{E}-13$ & 4.96 & fold increase during starvation \\
\hline Trib3 & 2.78 & 2.55 & 1.30E-26 & $2.61 \mathrm{E}-24$ & 6.86 & fold increase during starvation \\
\hline Ttbk1 & -1.76 & 0.42 & $2.97 \mathrm{E}-10$ & $1.85 \mathrm{E}-08$ & 3.38 & fold increase during refeeding \\
\hline Ttc25 & -2.53 & -0.86 & $3.16 \mathrm{E}-14$ & $3.02 \mathrm{E}-12$ & 5.77 & fold increase during refeeding \\
\hline Ttn & 1.47 & 2.36 & 2.21E-09 & $1.23 \mathrm{E}-07$ & 2.76 & fold increase during starvation \\
\hline Uap1l1 & 1.51 & 3.14 & 4.25E-09 & $2.24 \mathrm{E}-07$ & 2.85 & fold increase during starvation \\
\hline Ubd & 2.41 & 0.93 & $8.12 \mathrm{E}-17$ & $1.02 \mathrm{E}-14$ & 5.33 & fold increase during starvation \\
\hline Ube2c & 1.59 & -0.77 & 3.98E-07 & 1.52E-05 & 3.02 & fold increase during starvation \\
\hline Ugt1a5 & -1.80 & 4.93 & $1.43 E-15$ & $1.70 \mathrm{E}-13$ & 3.48 & fold increase during refeeding \\
\hline Utp14b & -1.54 & 4.26 & $3.96 \mathrm{E}-12$ & $3.13 \mathrm{E}-10$ & 2.90 & fold increase during refeeding \\
\hline Vmn2r3 & 7.07 & 0.78 & $4.18 \mathrm{E}-60$ & $5.04 \mathrm{E}-57$ & 134.38 & fold increase during starvation \\
\hline Vnn1 & 2.20 & 5.92 & $1.04 \mathrm{E}-23$ & $1.99 \mathrm{E}-21$ & 4.60 & fold increase during starvation \\
\hline Wdr52 & 1.95 & -1.06 & $3.20 \mathrm{E}-08$ & $1.51 \mathrm{E}-06$ & 3.86 & fold increase during starvation \\
\hline Wfdc3 & 4.87 & -0.09 & $2.68 E-35$ & $7.90 \mathrm{E}-33$ & 29.14 & fold increase during starvation \\
\hline
\end{tabular}




\section{CHAPTER 5: SUMMARY}

Alternative splicing is a major source of protein diversity, yet the mechanisms regulating the process by exogenous factors remains largely unexplored. Our laboratory has identified RNA splicing as an intracellular target for nutrient regulation of gene expression. We have found that changes in the accumulation of G6PD mRNA is caused by changes in splicing in response to nutrient availability - specifically starvation and refeeding. The focus of my dissertation research was to enhance our understanding of the molecular mechanisms underlying the changes in pre-mRNA splicing that occur in response to nutrient signals in the liver.

Using RNA-immunoprecipitation in whole mouse liver, I demonstrated that the splicing factor SRSF3 binds specifically to G6PD mRNA in a nutrient-dependent manner, resulting in an enhancement of G6PD mRNA splicing. This was a novel finding in that SRSF3 binding to G6PD mRNA is significantly increased in the livers of refed mice versus starved mice, and that direct binding of SRSF3 to G6PD has never been described. We hypothesized that since G6PD is a critical enzyme in the lipogenic pathway, it was unlikely that regulation at the level of splicing was unique. To extend this paradigm, I performed RNA-seq on livers from mice that had been starved or starved and then refed. The goal was to identify additional genes that exhibited changes in alternative splicing in response to starvation and refeeding similar to those observed with G6PD, specifically intron retention. To our surprise, we did not identify any other genes whose frequency of intron retention was significantly affected by starvation or refeeding. However, we do not feel that these results completely nullify our hypothesis because we suspect that technical changes to the experimental design could yield more conclusive results (detailed in Chapter 4). Regulation of splicing by nutrients, particularly in 
liver, represents an important potential control mechanism for fine-tuning gene expression in a metabolic organ in which precision is key. Understanding this mechanism of regulation could be critical to solving several mysteries with regard to the onset and/or severity of metabolic dysfunction and disease. Thus, further studies are needed to definitively identify additional genes whose splicing patterns are regulated by nutrient status.

As we were conducting the RNA-seq studies, we also attempted to approach the question of splicing regulation from another direction. Since we had established hnRNP K and SRSF3 as the splicing factors responsible for regulating G6PD intron retention in liver (Chapter 2) in response to starvation and refeeding, respectively, we sought to identify additional target genes of both proteins in liver using a CLIP-Seq technique (Appendix I). Because expression of SRSF3 in primary rat hepatocytes is relatively low, we attempted to overexpress a FLAG-tagged SRSF3 protein using adenovirus in those cells. However, production of a functional SRSF3 adenovirus using standard techniques proved to be unexpectedly impossible. Specifically, I discovered that the adenovirus life cycle is inhibited by SRSF3 overexpression during the packaging phase. As a result, the majority of the work on this project was devoted to the development of novel adenovirus expression system to circumvent this problem (Chapter 3).

Our discovery that accumulation G6PD mRNA in liver is regulated by changes in intron retention in response to starvation and refeeding is very likely one example of many. While our RNA-seq data were inconclusive, the question of whether nutrient regulation of splicing is a major layer of control governing hepatic gene expression is critical to our understanding of cellular function. Furthermore, the role of the nutrient regulated splicing factor, SRSF3, has yet to be determined, but future studies in our laboratory will involve utilizing the tools we 
have developed to elucidate its role in liver and gain a more thorough understanding of nutrient-regulation of RNA splicing. 


\section{APPENDIX}

\section{CLIP-Seq Protocol}

\section{Hepatocyte Isolation}

Male Sprague-Dawley rats (180-200g, Harlan Laboratories, Indianapolis, IN) were fed a standard chow diet (Harlan Teklad). Rats were starved for 18 hours prior to hepatocyte isolation. Animals were sedated with isoflurane prior to surgery and hepatocytes were isolated by a modification of the technique of Seglen [1]. The livers were perfused with $350 \mathrm{ml}$ of calcium-free buffer (0.14 M NaCl, $6.7 \mathrm{mM} \mathrm{KCl,} 0.02 \mathrm{M}$ HEPES, pH 7.4, $25 \mathrm{mM}$ glucose, 250 $\mathrm{mM} \mathrm{EGTA})$ at $40 \mathrm{ml} / \mathrm{min}$ followed by $100 \mathrm{ml}$ of a buffer containing collagenase $(67 \mathrm{mM} \mathrm{NaCl}$, 6.7 mM KCl, 0.1 M HEPES, pH 7.4, $6 \mathrm{mM} \mathrm{CaCl}_{2} \bullet 2 \mathrm{H}_{2} \mathrm{O}, 25 \mathrm{mM}$ glucose, $1 \mathrm{mg} / \mathrm{ml}$ collagenase $\mathrm{H}, 0.05 \mathrm{mg} / \mathrm{ml}$ trypsin inhibitor). The hepatocytes were then teased from the capsid of the liver and washed into cold, oxygenated 1X Waymouth medium (Waymouth's MB752/1 supplemented with $26 \mathrm{mM} \mathrm{NaHCO}_{3}, 20 \mathrm{mM} \mathrm{HEPES} \mathrm{pH} \mathrm{7.4,} 0.5 \mathrm{mM}$ serine, $0.5 \mathrm{mM}$ alanine, penicillin $(100 \mathrm{U} / \mathrm{ml})$, streptomycin $(100 \mathrm{mg} / \mathrm{ml})$, and gentamicin $(50 \mathrm{mg} / \mathrm{ml})$, and $0.2 \%$ bovine serum albumin). The cells were spun through a Percoll gradient for $10 \mathrm{~min}$ at $50 \times \mathrm{g}$ to remove non-parenchymal cells. Hepatocytes $\left(3 \times 10^{6}\right)$ were plated in $60-\mathrm{mm}$ tissue culture dishes precoated with rat tail collagen in $1 \mathrm{X}$ Waymouth medium containing $5 \%$ newborn calf serum. Cell viability in all experiments was $90 \%$ or greater as estimated by Trypan Blue (0.4\%) exclusion. After $4 \mathrm{~h}$, the cells were washed twice with $1 \mathrm{X}$ serum-free Waymouth medium. A Matrigel overlay $(0.3 \mathrm{mg} / \mathrm{ml})$ was added and the cells incubated overnight $\left(37^{\circ} \mathrm{C}, 5 \% \mathrm{CO}_{2}\right)$. The next morning, the cells were washed twice with $1 \mathrm{X}$ serum-free Waymouth medium and treated with $80 \mathrm{nM}$ insulin for $24 \mathrm{~h}$ prior to lysate preparation. 


\section{Lysate Preparation and UV Crosslinking}

The monolayer was washed once with $37^{\circ} \mathrm{C} 1 \mathrm{x}$ PBS, and then covered with cold $1 \mathrm{x}$ PBS. Half of the plates were exposed to $400 \mathrm{~mJ} / \mathrm{cm}^{2}$ of UV light in a Stratalinker ${ }^{\circledR}$ (Stratagene, La Jolla, CA), and the other half used as the non-crosslinked ("-UV") control. The cells were scraped off the plates and centrifuged at $5000 \mathrm{rpm}$. The supernatant was aspirated and the pellets resuspended in 2 volumes of lysis buffer ( $1 \times$ PBS, $0.1 \%$ SDS, $0.5 \%$ sodium deoxycholate, and $0.5 \%$ Nonidet P-40 in DEPC-treated water). Following lysis, the samples (both + and - UV) were homogenized by passing through a QIAShredder ${ }^{\circledR}$ (Qiagen, Valencia, CA) spin column two times each. The homogenized lysates were stored in small aliquots at $80^{\circ} \mathrm{C}$.

\section{Immunoprecipitation of SRSF3}

The immunoprecipitation is a modification of existing methods [2-4]. $500 \mathrm{ug}$ of homogenized lysate was pre-cleared in 100uL lysis buffer containing RNAse I, SuperRNAse IN (Life Technologies), and Halt $\circledast$ Protease/Phosphatase Inhibitor Cocktail (Thermo Fisher, Waltham, MA), and 0.5mg Protein A/G Dynabeads (Life Technologies). The mixture was rotated for $1 \mathrm{~h}$ at $4^{\circ} \mathrm{C}$.

$3 \mathrm{mg}$ of Protein A Dynabeads (Life Technologies, Carlsbad, CA) were washed 3 times in bead wash buffer (1x PBS pH 7.4, 0.02\% Tween 20). The beads were resuspended in 100uL bead wash buffer containing 5 ug of mouse anti-SRp20(C-1) antibody (SCBT, Santa Cruz, CA). Bead:antibody complexes were allowed to form by incubating at room temperature for 30 minutes with end-over-end rotation.

Following incubation, the bead:antibody complexes were washed $3 x$ with bead wash buffer and resuspended in the pre-cleared lysate. The immunoprecipitation was performed at 4 
${ }^{\circ} \mathrm{C}$ for 2 hours with end-over-end rotation. After incubation, supernatants were saved for future analysis. The complexes were washed under stringent conditions [1x high stringency wash buffer, then $1 x$ high salt wash buffer, then $2 x$ low salt wash buffer). An aliquot equal to $5 \%$ of the IP volume was saved for future analysis after the last wash step. The beads were washed twice in 1x Polynucleotide kinase buffer (PNK buffer; 50mM Tris- $\mathrm{HCl} \mathrm{pH} \mathrm{7.4,} 10 \mathrm{mM} \mathrm{MgCl}$, $0.5 \% \mathrm{v} / \mathrm{v}$ Nonidet P-40).

\section{Dephosphorylation and 3' Linker Ligation}

All traces of PNK buffer were removed and the beads were resuspended in Antarctic Phosphatase mix (New England Biolabs) with 20 units of SuperRNAse IN. The reactions were incubated for 20 minutes at $37^{\circ} \mathrm{C}$ with mild agitation. Samples were washed once in $1 \times$ PNK buffer, then once in 1x PNK+EGTA buffer (50 mM Tris-HCl pH 7.4, $20 \mathrm{mM}$ EGTA, 0.5\% v/v Nonidet P-40). Samples were transferred to fresh tubes and washed twice more with $1 \mathrm{x}$ PNK buffer. The bead:lysate complexes were resuspended in $80 \mathrm{uL}$ of 3 ' linker mixture* containing T4 RNA ligase (Thermo Scientific). The samples were incubated overnight at $16^{\circ} \mathrm{C}$ on a slow shaker. *3' Linker Sequence: 5'-GUGUCAGUCACUUCCAGCGG-PMN-3'

\section{RNA:RNABP Complex Purification}

Following overnight linker ligation, the samples were washed once with 1x PNK buffer, once with 5x PNK buffer, and twice with $1 x$ PNK buffer. The samples were resuspended in T4

polynucleotide kinase reaction mix [0.8uL PNK buffer, 0.4uL T4 PNK, 0.8uL [Y $\left.{ }^{-32}{ }^{32}\right]-A T P(3000$ $\mathrm{Ci} / \mathrm{mmol}$ ) to final volume of $8 \mathrm{uL}$ (New England Biolabs). The samples were incubated at $37^{\circ} \mathrm{C}$ for 10 minutes. $1 \mathrm{uL}$ of $10 \mathrm{mM}$ cold ATP was added to each sample and incubated for an additional 5 minutes at $37^{\circ} \mathrm{C}$. The samples were then washed once in $1 \times$ PNK buffer, once in 
5x PXL (1x PBS, 0.1\% SDS, 0.5\% sodium deoxycholate, 0.5\% Nonidet P-40), and twice in 1x PNK. The samples were resuspended 1:1 in 2x NuPAGE LDS sample buffer with reducing agent (Life Technologies) and heated at $70^{\circ} \mathrm{C}$ for 10 minutes. Next, the beads were magnetically captured and the supernatants run on a NuPAGE $4-12 \%$ Bis-Tris gel (Life Technologies) at 165 volts for 45 minutes at $4^{\circ} \mathrm{C}$. The protein:RNA complexes were transferred onto a Protran BA85 nitrocellulose membrane (Whatman). The membrane was washed once in $1 \mathrm{x}$ PBS, and wrapped in plastic wrap. The membrane was exposed to film at $-80^{\circ} \mathrm{C}$ for 2 hours.

\section{Protein Digestion and 5' Linker Ligation}

For each sample, 200uL of Proteinase K buffer containing $4 \mathrm{mg} / \mathrm{mL}$ Proteinase K (Life Technologies) was prepared and warmed in a $37^{\circ} \mathrm{C}$ water bath for 20 minutes. The RNABP:RNA complexes corresponding to a band located approximately 20kD above SRp20 was excised from the membrane with a clean scalpel (roughly $35-45 \mathrm{kD}$ ). $200 \mathrm{uL}$ of the warmed Proteinase $\mathrm{K}$ solution was added to the sliced membrane and incubated at $37^{\circ} \mathrm{C}$ for 20 minutes. Next, 200uL of freshly prepared 1x PK Buffer/Urea (100 mM Tris-HCL pH 7.5, 50 mM $\mathrm{NaCl}, 10 \mathrm{mM}$ EDTA, and $7 \mathrm{M}$ Urea) was added to each sample and incubated for an additional 20 minutes at $37^{\circ} \mathrm{C}$. To extract the liberated RNAs, 400uL water-saturated phenol and $130 \mathrm{uL}$ chloroform:isoamyl alcohol (24:1) was added to each sample and then incubated for 20 minutes at $37^{\circ} \mathrm{C}$. The samples were centrifuged at max speed for 5 minutes and the aqueous phases transferred to fresh $1.5 \mathrm{~mL}$ tubes. $0.75 \mathrm{uL}$ GlycoBlue (Ambion), 50uL of 3M NaOAc $(\mathrm{pH}$ 5.2) and $1 \mathrm{~mL}$ of ethanol:isopropanol (1:1) was added to aqueous phase and the RNA precipitated overnight at $-20^{\circ} \mathrm{C}$. The samples were centrifuged at max speed for 25 minutes at $4^{\circ} \mathrm{C}$. The RNA pellets were washed twice with ice-cold $75 \%$ ethanol and then air-dried on the 
bench top for 5-10 minutes. The RNA pellets were resuspended in 6.9uL RNAse-free water. Finally, 3.1uL of 5'-linker mixture (1x T4 RNA Ligase Buffer, 1ug BSA, 1 unit T4 RNA Ligase, and 20pmol 5'-linker ${ }^{\star}$ ) was added to the RNA and incubated overnight at $16^{\circ} \mathrm{C}$ on a slow shaker.

`5' Linker Sequence: 5’-AGGGAGGACGAUGCGG-3'

\section{Reverse Transcription and cDNA Synthesis}

95uL of Turbo DNAse reaction mixture (Ambion) and 5uL of SuperRNAse IN were added to the RNA samples and incubated at $37^{\circ} \mathrm{C}$ for 20 minutes. To precipitate the RNA, 300uL of water-saturated phenol, 300uL RNAse-free water, and 100uL chloroform:isoamyl alcohol (24:1) was added to each sample. The samples were vortexed, then centrifuged at max speed for 5 minutes at room temperature. The aqueous phase was transferred to a fresh tube and $300 \mathrm{uL}$ chloroform:isoamyl alcohol was added to each sample. The samples were again centrifuged at room temperature for 2 minutes at max speed. The aqueous phase was transferred to a fresh tube. 0.5uL GlycoBlue, 50uL $3 \mathrm{M} \mathrm{NaOAc}(\mathrm{pH} 5.2)$, and $1 \mathrm{~mL}$ ethanol:isopropanol (1:1) was added to each sample and the RNA precipitated overnight at $20^{\circ} \mathrm{C}$. The samples were centrifuged at max speed for 25 minutes at $4^{\circ} \mathrm{C}$. The RNA pellets were washed twice with ice-cold $75 \%$ ethanol and then air-dried on the bench top for 5-10 minutes. The RNA pellets were resuspended in 18uL of RNAse-free water and divided equally for $+/$ - reverse transcriptase cDNA synthesis reactions. 10pmol of DP3 primer* and $1 \mathrm{mM}$ dNTPs were added to each tube and incubated at $65^{\circ} \mathrm{C}$ for 5 minutes. During the incubation, reverse transcription reaction mixtures were prepared (1x SuperScriptIII RT buffer, 1mM dNTPs, 100mM DTT, 20 units SuperRNAse IN, and 200 units SuperScriptIII (+RT) or water (RT)). 8uL of reverse transcriptase reaction mixture was added to the appropriate tubes and 
subjected to the following thermal cycler conditions: $50^{\circ} \mathrm{C}$ for 45 minutes, $55^{\circ} \mathrm{C}$ for 15 minutes, $90^{\circ} \mathrm{C}$ for 5 minutes, and a $4^{\circ} \mathrm{C}$ infinite hold.

*DP3 Primer Sequence: 5'-CCGCTGGAAGTGACTGACAC-3'

\section{$1^{\text {st }}$ PCR Amplification and Gel Analysis}

25uL PCR mixtures were prepared as follows: 1x OneTaq PCR MasterMix (New England Biolabs), 0.4uM DP3 primer, 0.4uM DP5* primer and 8.5uL cDNA. Polymerase activation was performed at $94^{\circ} \mathrm{C}$ for 30 seconds, followed by 30 cycles of $94^{\circ} \mathrm{C}$ for 20 seconds, $58^{\circ} \mathrm{C}$ for 30 seconds, and $68^{\circ} \mathrm{C}$ for 30 seconds. A final extension step at $68^{\circ} \mathrm{C}$ for 5 minutes was followed by a $4^{\circ} \mathrm{C}$ infinite hold.

2x gel loading buffer was added (1:1) to each PCR prior to loading on a 10\% denaturing PAGE gel. The gel was run at $300 \mathrm{~V}$ until the bromophenol blue dye front reached the bottom of the gel. After running, the gel plates were disassembled and the gel immersed in a 1:10,000 solution of SYBR Gold in 1x TBE for 30 minutes. PCR products were visualized on a UV transilluminator with an excitation wavelength of 312-nm to minimize photonicking/photodimerization of the DNA. The PCR products running between $\sim 75-100 \mathrm{bp}$ were excised from the gel and transferred to clean $1.5 \mathrm{~mL}$ microcentrifuge tubes. The $-\mathrm{RT}$ lanes did not contain any product, indicating that our samples were not contaminated with DNA from previous steps. The DNA was extracted from the gel slices using the QIAQuick Gel Purification Kit with the Qiagen-supplied "user-developed" protocol for polyacrylamide gels, and eluted in a final volume of 30uL nuclease-free water. *DP5 Primer Sequence: 5'-AGGGAGGACGATGCGG-3' 


\section{Sequencing Preparation PCR}

2uL of DP3/DP5-amplified PCR product was added to PCR mixtures prepared as follows: 1x OneTaq PCR MasterMix (New England Biolabs), 0.4uM Salati_FP3 primer, 0.4uM Salati_FP5* primer, and water to a final reaction volume of $25 \mathrm{uL}$. Polymerase activation was performed at $94^{\circ} \mathrm{C}$ for 30 seconds, followed by 6 cycles of $94^{\circ} \mathrm{C}$ for 20 seconds, $65^{\circ} \mathrm{C}$ for 30 seconds, and $72^{\circ} \mathrm{C}$ for 30 seconds. A final extension step at $72^{\circ} \mathrm{C}$ for 5 minutes was followed by a $4^{\circ} \mathrm{C}$ infinite hold.

2x gel loading buffer was added (1:1) to each PCR prior to loading on a 10\% denaturing PAGE gel. The gel was run at $300 \mathrm{~V}$ until the bromophenol blue dye front reached the bottom of the gel. After running, the gel plates were disassembled and the gel immersed in a 1:10,000 solution of SYBR Gold in 1X TBE for 30 minutes. PCR products were visualized on a UV transilluminator with an excitation wavelength of 312-nm to minimize photonicking/photodimerization of the DNA. The PCR products running between $\sim 200-250 \mathrm{bp}$ were excised from the gel and transferred to clean $1.5 \mathrm{~mL}$ microcentrifuge tubes. The DNA was extracted from the gel slices using the QIAQuick Gel Purification Kit with the Qiagen-supplied "user-developed" protocol for polyacrylamide gels, and eluted in a final volume of 30uL nuclease-free water as before. Recovered PCR products were analyzed for quality with a High Sensitivity DNA Assay on a 2100 Bioanalyzer (Agilent Technologies, Santa Clara, CA).

Samples were then submitted for library preparation and deep sequencing using an Illuminabased platform.

*Salati_FP3 Primer Sequence: 5'-CAAGCAGAAGACGGCATACGAGATAAGGCCGTGACTGG AGTTCAGACGTGTGCTCTTCCGATCTCCGCTGGAAGTGACTGACAC-3'

*Salati_FP5 Primer Sequence: 5'-AATGATACGGCGACCACCGAGATCTACACTCTTTCCCTA CACGACGCTCTTCCGATCTCTATGGATACTTAGTCAGGGAGGACGATGCGG-3' 


\section{References}

1. Seglen, P.O., Preparation of isolated rat liver cells. Methods Cell Biol, 1976. 13: p. 2983.

2. Darnell, J.C.M., A.; Hung, K.Y.S.,; Darnell, R.B., Mapping of In Vivo RNA-Binding Sites by UV-Cross-Linking Immunoprecipitation (CLIP), in MolecuLar Cloning: A Laboratory Manual (4th Edition). 2012, Cold Spring Harbor Laboratory Press: Cold Spring Harbor, NY. p. 1703-1759.

3. Darnell, R., CLIP (cross-linking and immunoprecipitation) identification of RNAs bound by a specific protein. Cold Spring Harb Protoc, 2012. 2012(11): p. 1146-60.

4. Ule, J., et al., CLIP: a method for identifying protein-RNA interaction sites in living cells. Methods, 2005. 37(4): p. 376-86. 


\section{Isolation of Protein \& RNA From Cells in Culture}

\section{Sample Collection \& Protein Isolation}

1. Remove excess media from monolayer, leaving behind approximately $1 \mathrm{~mL}$.

2. With the cells still in $1 \mathrm{~mL}$ of their regular media, scrape cells off the bottom of the wells.

3. Transfer cells into a clean $1.5 \mathrm{~mL}$ epi tube.

4. Pipet cells gently to ensure that they are in a relatively homogenous suspension.

*Use a wide mouth pipet tip to avoid damaging the cells.

5. Transfer half (about 500uL) of the cell suspension to a separate $1.5 \mathrm{~mL}$ epi tube.

6. Centrifuge both tubes at $4^{\circ} \mathrm{C}, 2$ minutes at $2000 \mathrm{RPM}$

(NOT MAX SPEED! Low speed is important to maintain the integrity of the cell- spinning too fast will result in premature lysis of your cells.)

7. Decant or pipet off supernatant from each pellet. Samples should be on ice as much as possible.

8. Rinse protein tubes with 100uL PBS and decant.

9. Add 150uL 1x RIPA buffer (+Protease inhibitor cocktail) to the pellet.

10. Sonicate each sample with the following settings:

Duty: 50

Output: 2

10 pulses per sample

11. Centrifuge lysates for 10 minutes at $4^{\circ} \mathrm{C}$ (max speed), and transfer supernatant to clean tube and proceed to $\mathrm{BCA}$ assay or store at $-80^{\circ} \mathrm{C}$.

12. For RNA, rack the tubes to loosen the pellet and immediately add 500uL TRI-reagent reagent. If the pellet looks to be larger than $\sim 50 \mathrm{uL}$, add $1 \mathrm{~mL}$ Tri-reagent. Vortex well to mix. Freeze at $-20^{\circ} \mathrm{C}$ or proceed to RNA extraction protocol.

\section{B. RNA Isolation}

1. Thaw samples in TRI-Reagent at room temperature 
2. Add $300 \mathrm{uL}$ chloroform per $1 \mathrm{~mL}$ of TRI-reagent sample. Shake vigorously by hand for 15-30 seconds.

3. Incubate tubes at room temp for 5-15 minutes.

4. Centrifuge samples at 12,000 RPM for 15 minutes at $4^{\circ} \mathrm{C}$. During centrifugation, label fresh $1.5 \mathrm{~mL}$ tubes.

5. Carefully transfer the upper clear phase $(\sim 500 \mathrm{uL})$ into a fresh labeled tube.

6. Add an additional $300 \mathrm{uL}$ of chloroform to each sample and centrifuge again at 12,000 RPM for 15 minutes at $4^{\circ} \mathrm{C}$. During centrifugation, label fresh $1.5 \mathrm{~mL}$ tubes.

7. Carefully transfer the upper clear phase $(\sim 500 \mathrm{uL})$ into a fresh labeled tube. Use extra caution with this second collection of the aqueous layer as the interface is harder to see.

8. Add $\sim 400$ uL isopropanol (want a 1:1 ratio) to each tube, cap, and mix by inversion several times.

9. Incubate at room temperature for 5-10 minutes.

10. Centrifuge samples at 12,000 RPM for 8 minutes at $4^{\circ} \mathrm{C}$. The RNA precipitate will form a pellet.

11. Decant the supernatant, being careful not to disturb the pellet. If the pellet becomes loose, centrifuge again for 5 minutes.

12. Wash the pellet with $1 \mathrm{~mL}$ chilled $75 \%$ ethanol.

The samples can be stored at this point for up to 1 year at $-20^{\circ} \mathrm{C}$.

13. Centrifuge the samples at 8000 RPM for 5 minutes at $4^{\circ} \mathrm{C}$.

14. Decant the supernatant, being careful not to disturb the pellet. If it comes loose, repeat centrifugation.

15. Carefully pipet off excess liquid and/or blot dry with a KimWipe. DO NOT TOUCH THE PELLET!

16. Let the pellet air-dry for 2-3 minutes. 
17. Rehydrate the pellet with 44uL RNAse-free water.

18. Incubate at $55-60^{\circ} \mathrm{C}$ for 10 minutes and proceed to DNAse treatment.

C. DNAse Treatment

1. Prepare a master mix of Turbo DNAse (Ambion kit).

For each sample, you will need:

5uL 10X Turbo DNAse Buffer

1uL Turbo DNAse

2. Add 6uL of the prepared DNAse master mix to each RNA sample (for a 50uL total volume), mix by flicking or pulse vortexing.

3. Incubate at $37^{\circ} \mathrm{C}$ for $20-30$ minutes.

During this time, begin thawing the DNAse Inactivation Reagent and label fresh tubes.

4. Vortex DNAse Inactivation Reagent immediately before use, then add $5 \mathrm{uL}$ to each sample and incubate at RT for 5 minutes.

Flick tubes periodically to keep the solution mixed.

5. Centrifuge samples at 10,000 RPM for 1.5 minutes at RT. A white pellet will form.

6. Transfer the aqueous layer to a fresh tube.

Be careful not to disturb the pellet.

7. Store RNA at $-80^{\circ} \mathrm{C}$ until ready to use and avoid frequent freeze/thaws. 


\section{RNA Immunoprecipitation (RIP) Protocol}

\section{A. Lysate preparation}

a. Crosslink cells by adding $37 \%$ formaldehyde to a final concentration of $1 \%$ and incubate in the hood for 10 minutes.

b. During incubation, prepare RIPA++ Lysis Buffer:

- Per 100uL:

6uL RNAsin

1uL 100x PI Cocktail

1uL 100x 0.5M EDTA

92uL RIPA Buffer
For

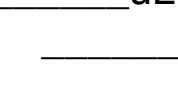

UL:

uL RNAsin

uL 100x PI Cocktail UL 100x 0.5M EDTA uL RIPA Buffer

c. Stop crosslinking reaction by adding $2 \mathrm{M}$ glycine to a final concentration of $200 \mathrm{mM}$ and incubate in the hood for 5-10 minutes.

d. Wash plates twice with $10 \mathrm{~mL}$ of ice-cold PBS.

e. Add uL of RIP Lysis Buffer. Scrape cells off each plate and transfer to a centrifuge tube.

f. Aliquot the lysate for BioRupter sonication: 100uL-300uL lysate per 1.5mL Epi tube.

g. Sonicate lysate using the following conditions: $2 \times 15$ min. cycles on medium intensity with 30 s on/off pulses. Change ice bath between the 2 cycles.

h. Centrifuge sonicated lysate at $4^{\circ} \mathrm{C}$ at $10,000 \mathrm{RPM}$ and transfer supernatant to fresh tubes.

i. Quantify protein using BCA assay and record concentrations:

B. Prepare beads and lysate for immunoprecipitation

\begin{tabular}{|c|c|c|c|c|c|}
\hline Sample & $\begin{array}{c}\text { [Protein] } \\
\text { ug/uL }\end{array}$ & $\begin{array}{c}\text { uL needed } \\
\text { for 500ug }\end{array}$ & uL Antibody & uL PBS++ & $\begin{array}{c}\text { Total IP } \\
\text { Volume }\end{array}$ \\
\hline & & & & & \\
\hline & & & & & \\
\hline
\end{tabular}

\section{Pre-Clear Lysate:}

a. Add 20uL of beads to each tube for IP.

b. Wash 2x with PBS/T wash buffer (RNAse-free PBS with $0.02 \%$ Tween) 
c. Add the specified amount of each lysate (see above) and PBS++ IP Buffer to each tube.

d. Incubate at $4^{\circ} \mathrm{C}$ with rotation for $1 \mathrm{~h}$.

PBS++ IP Buffer:

Per 100uL:

1uL 100x PI Cocktail

For uL:

1uL 100x 0.5M EDTA

6uL RNAsin

92uL RNAse-Free PBS

uL 100x PI Cocktail

UL 100x 0.5M EDTA

UL RNAsin

uL RNAse-Free PBS

e. Completely disperse and re-suspend magnetic beads by end-over-end rotation.

f. Label the appropriate number of tubes for each IP. Don't forget controls!!

g. Transfer $1 \mathrm{mg}$ beads (33uL) per $4 \mathrm{ug}$ of antibody to each tube.

h. Wash beads $2 x$ with $300 u L ~ 0.02 \%$ PBS/T (Wash Buffer).

i. Place tubes on a magnetic separator and discard the supernatant.

j. Remove the tubes from the magnet and re-suspend the beads in 200uL of wash buffer.

k. Add the indicated amount of each antibody to the appropriate tubes.

I. Incubate with rotation for 60 minutes at room temperature.

m. Centrifuge tubes briefly and place tubes on the magnet.

n. Remove supernatant and add $0.5 \mathrm{~mL}$ wash buffer to each tube.

o. Vortex briefly, then put tubes back on the magnet and remove the supernatant.

p. Repeat the last 2 steps for one additional wash.

q. Remove the tubes from the magnet and add $0.5 \mathrm{~mL}$ wash buffer to each magnet and vortex briefly. Place tubes on ice.

C. Immunoprecipitation of RNA-protein complexes (RIP)

a. Remove supernatant from bead-antibody complexes

b. Add pre-cleared lysate to the appropriate tube containing bead-antibody complexes. DO NOT FORGET TO SAVE AT LEAST ONE 10\% INPUT SAMPLE! 


\section{Incubate all tubes with rotation overnight at $4^{\circ} \mathrm{C}$.}

d. Centrifuge the tubes briefly and place on the magnetic separator. Transfer the supernatant to a new tube and save. ${ }^{*}$ Turn on the $55^{\circ} \mathrm{C}$ shaker ${ }^{*}$

e. Add $0.5 \mathrm{~mL} \mathrm{PBS} / \mathrm{T}$ wash buffer and vortex briefly.

f. Place tubes on magnet and discard the supernatant.

g. Repeat the above two steps 5 times, for a total of six washes.

h. Remove 50uL each out of the 500uL bead suspension during the last wash to test the efficiency of the IP by Western blot.

\section{Purification of RNA}

a. Prepare proteinase $\mathrm{K}(\mathrm{PK})$ buffer in the order below: Proteinase K (PK) Buffer:

Per 150uL:

126uL RNAse-Free PBS

15uL RNAse-Free $10 \%$ SDS

9uL $20 \mathrm{mg} / \mathrm{mL}$ Proteinase K

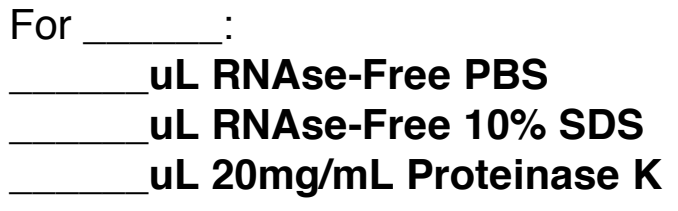

For

uL RNAse-Free PBS

$\mathrm{uL} 20 \mathrm{mg} / \mathrm{mL}$ Proteinase $\mathrm{K}$

a. Pull INPUT samples from section 3 and allow to thaw on ice.

b. Re-suspend each IP and input sample in 150uL of PK buffer.

C. Incubate all tubes for 30 minutes at $55^{\circ} \mathrm{C}$ with shaking.

d. Following PK digestion, centrifuge the tubes briefly.

e. DO NOT REMOVE ANY LIQUID!!!!! Add $1 \mathrm{~mL}$ TriReagent directly to each tube.

Proceed with RNA extraction and DNase treatment with the following modifications.

- Add 2uL GlycoBlue to the isopropanol wash step of the RNA extraction.

- For DNAse treatment, resuspend RNA pellet in 16uL of RNAse-free water.

- For each sample, add 2uL 10x Buffer, 1uL DNAse I, and 1uL Super RNAsin.

- Incubate in $37^{\circ} \mathrm{C}$ water bath for 25 minutes.

- Add 2uL DNAse Inactivation Reagent and incubate at RT for 5 mins.

- Centrifuge at max speed for 2 mins at RT.

- Transfer supernatants to freshly labeled tubes and keep on ice.

- For RT-PCR, add 7.8uL RNA to 12.2uL SYBR Master Mix with and without reverse transcriptase enzyme. 